\title{
Distribution of Mycobacterium avium subspecies paratuberculosis in clinically asymptomatic bulls and different non-ruminant species
}

\author{
Dissertation \\ to obtain the Ph. D. degree \\ in the Ph. D. Program for Agricultural Sciences in Göttingen (PAG) \\ at the Faculty of Agricultural Sciences, \\ Georg-August-University Göttingen, Germany
}

presented by

Kim Fechner

born in Celle, Germany

Göttingen, May 2017 
D7

1. Name of supervisor: Prof. Dr. Dr. Claus-Peter Czerny

2. Name of co-supervisor: Prof. Dr. Jürgen Hummel

Date of dissertation: $13^{\text {th }}$ July 2017 
TABLE OF CONTENS

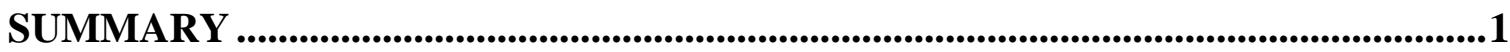

1 GENERAL INTRODUCTION

MYCOBACTERIUM AVIUM SUBSP. PARATUBERCULOSIS (MAP) ...................... 5

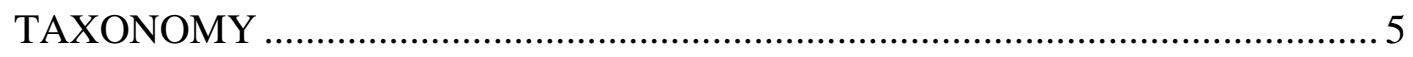

PATHOGEN CHARACTERIZATION AND TENACITY …............................. 8

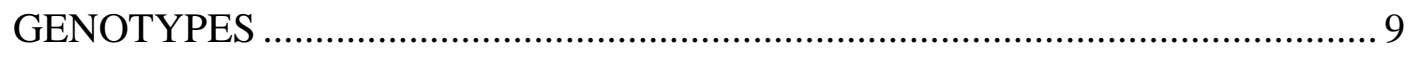

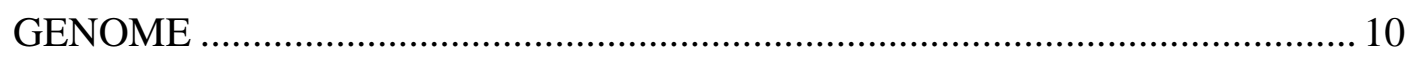

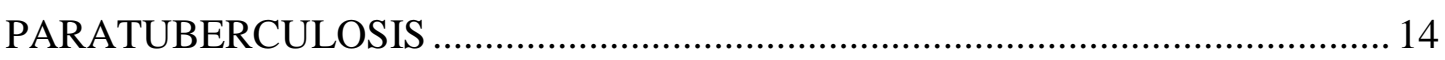

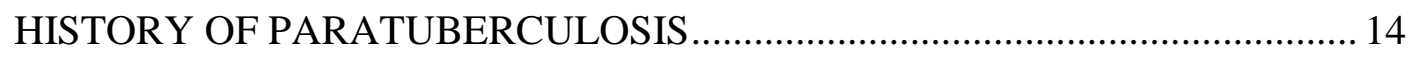

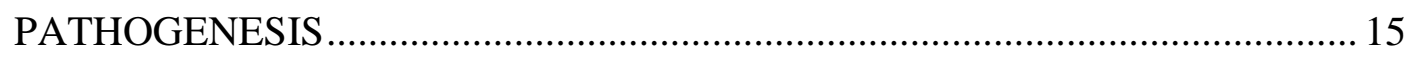

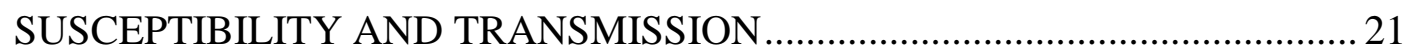

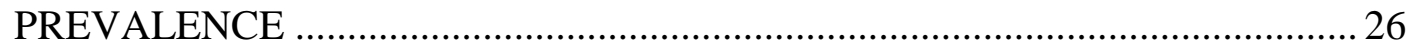

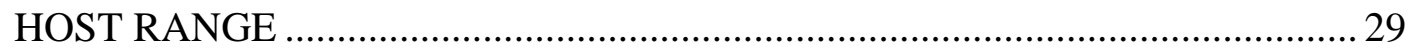

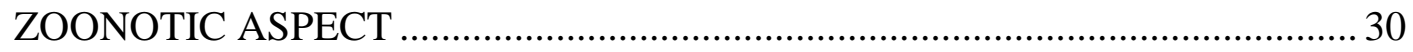

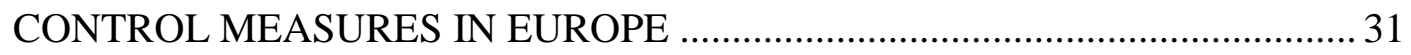

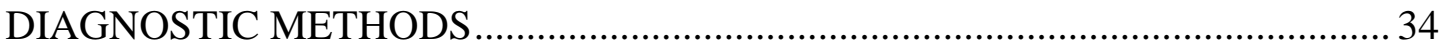

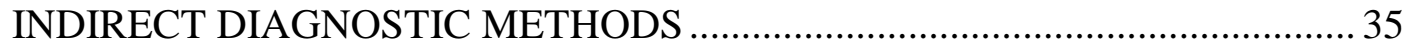

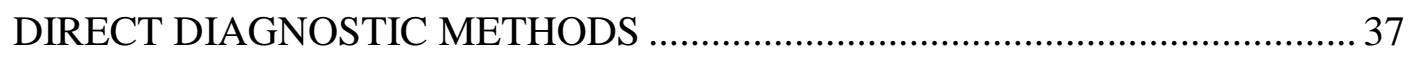

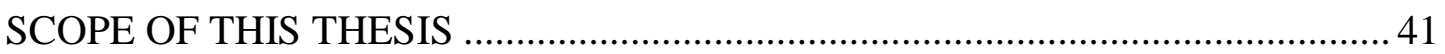

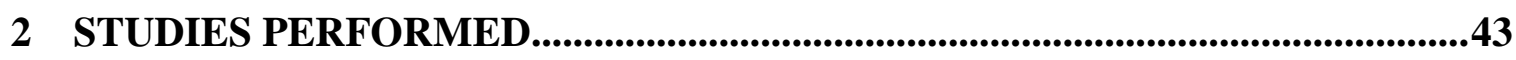

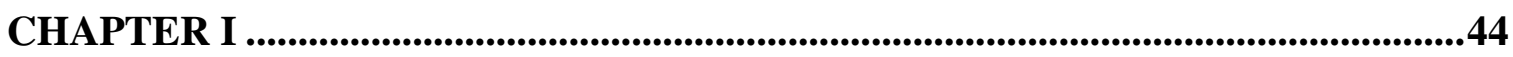

Distribution of Mycobacterium avium subsp. paratuberculosis in a Subclinical Naturally Infected German Fleckvieh Bull ................................................................44

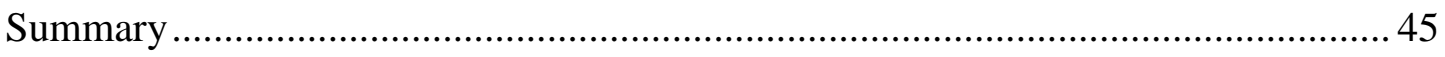

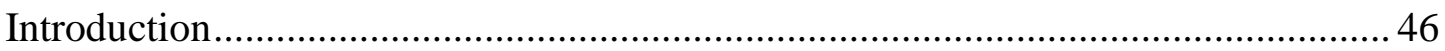




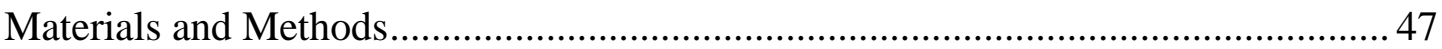

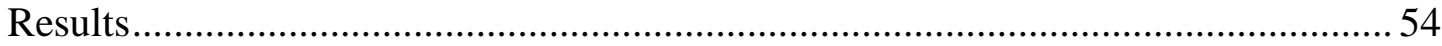

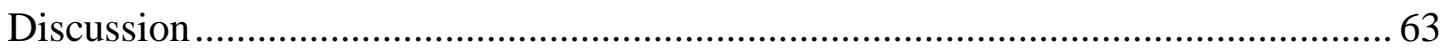

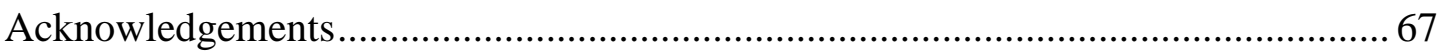

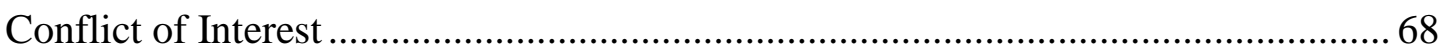

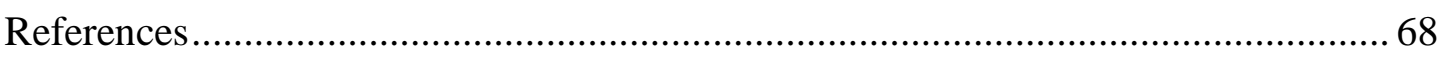

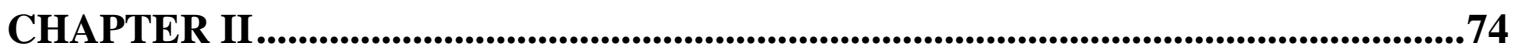

Detection of Mycobacterium avium subsp. paratuberculosis by IS900 based PCR assays in non-human primates kept in Germany ...........................................................74

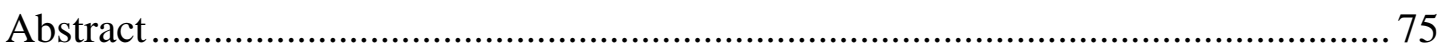

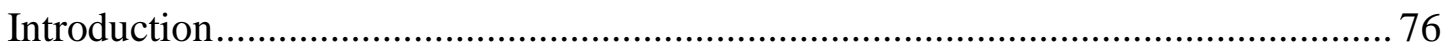

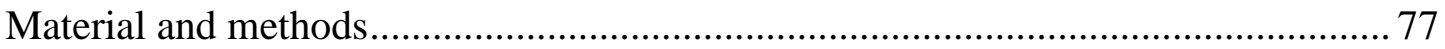

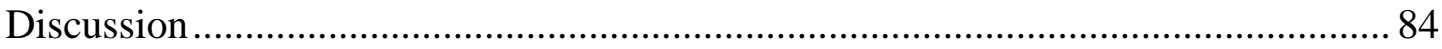

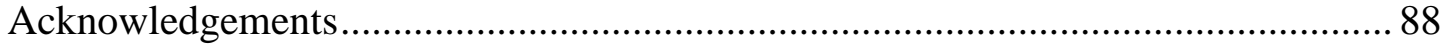

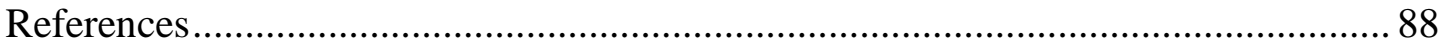

CHAPTER III ................................................................................................................................992

Detection of Mycobacterium avium subsp. paratuberculosis in rock hyraxes (Procavia capensis) imported from South Africa ........................................................92

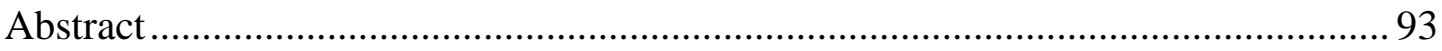

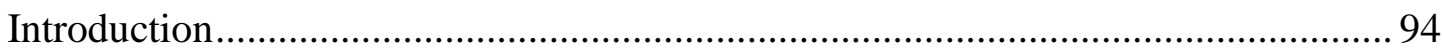

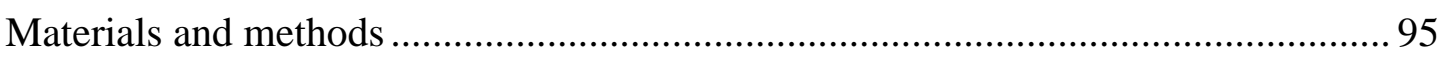

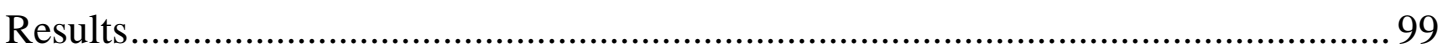

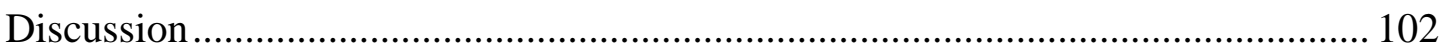

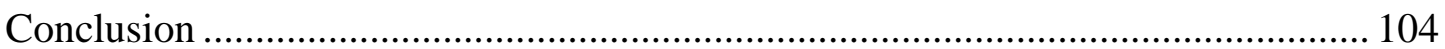

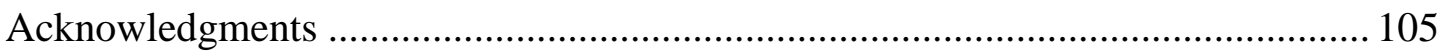

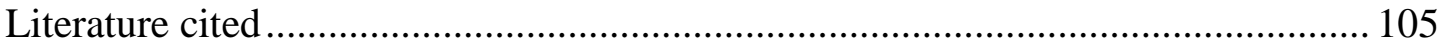

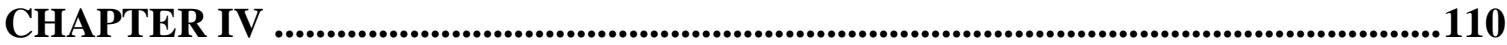


Development of a recombinase polymerase amplification assay for rapid detection of Mycobacterium avium subsp. paratuberculosis .........................................110

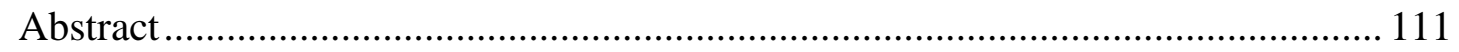

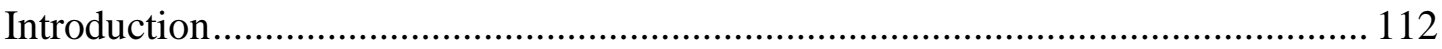

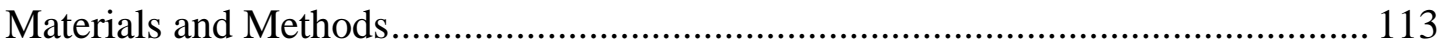

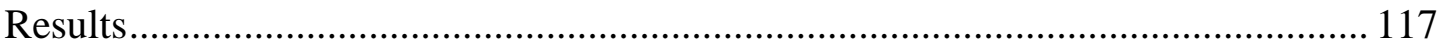

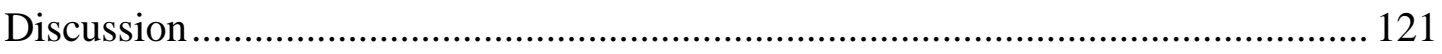

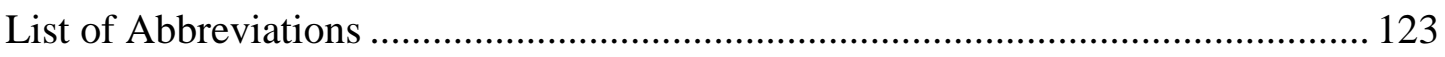

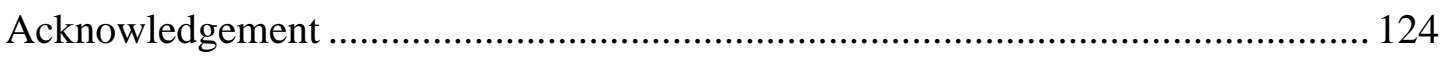

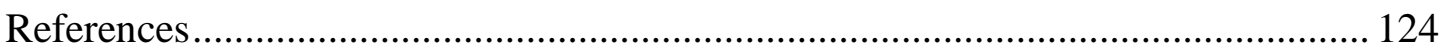

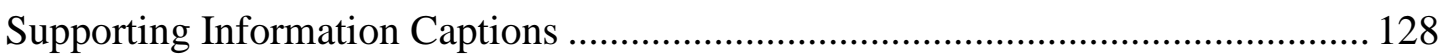

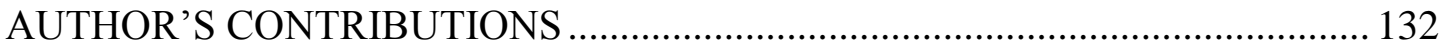

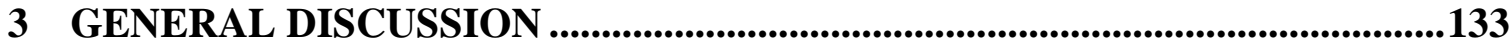

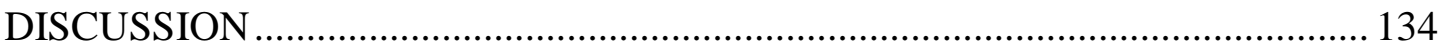

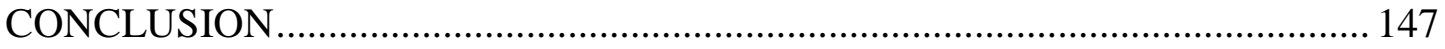

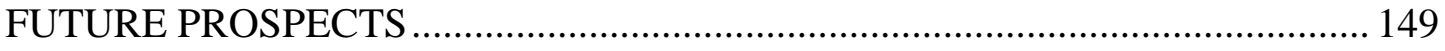

4 REFERENCES.....................................................................................................150

5 APPENDIX

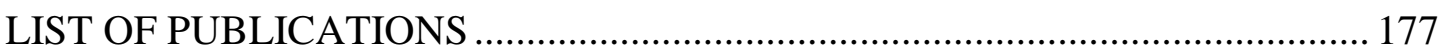

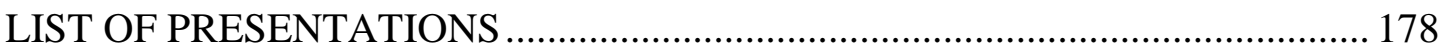

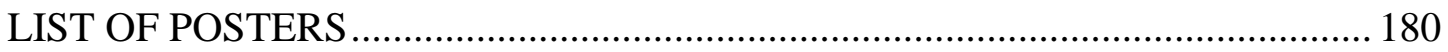

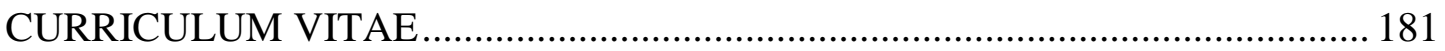

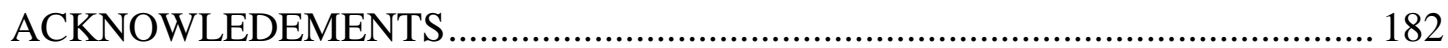

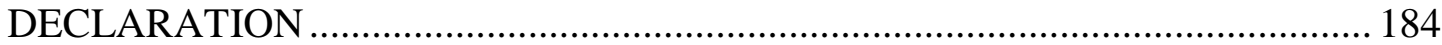




\section{SUMMARY}

Paratuberculosis is a disease of global distribution, which mainly occurs in ruminants. The disease is triggered by Mycobacterium avium subsp. paratuberculosis (MAP), which causes a chronic, degenerative granulomatous inflammation of the intestinal tract, in turn leading to diarrhoea, weight loss, reduced reproductive performance, and finally death. The economic losses sustained to the agricultural sector, as well as the potential aetiological link between MAP and Crohn's disease in humans, are the main reasons for the indispensability of an efficient control of paratuberculosis. The main challenge in ensuring efficient disease control is that infected animals do not necessarily show clinical symptoms and diagnostic methods are not reliable at this stage of the disease. To examine the unrecognized spread of asymptomatically infected hosts as well as potential transmission pathways, three studies were conducted in this thesis. In an additional study, a recombinase polymerase amplification (RPA) assay was developed to enable the detection of MAP in low-resource settings or directly in the stable.

The occurrence of MAP in the faeces, blood, and semen of a naturally infected, but clinically asymptomatic German Fleckvieh bull (related to Simmental: Bos primigenius taurus) was analysed over a period of four years, from the age of seven weeks until necropsy. MAP was intermittently detected by IS-900 based semi-nested PCR, real-time PCR, and bacteriological cultivation, with occurrence of MAP-free intervals of up to nine weeks. A recurring detection pattern was not found. Besides antigen detection, most of the implemented control programmes are based on the detection of an antibody response by ELISA. However, the examined bull was a serologically negative shedder over the entire investigation period. Such animals are one of the main reasons for the reduced success of paratuberculosis control programmes.

As well as ruminants, non-ruminants can be infected with MAP, but currently the knowledge concerning potential hosts is incomplete. It has been known for many years that the faeces of infected non-ruminants contains concentrations of up to $10^{6}$ MAP cells/g and might therefore play a role in the transmission of MAP to ruminants, through shared habitats. In this thesis, the presence of MAP was confirmed in rock hyraxes (Procavia capensis); a common marmoset (Callithrix jacchus); and a cottontop tamarin (Saguinus oedipus). Three of the investigated rock hyraxes were captured in South Africa and their four descendants were subsequently born in a quarantine stable at a German zoological garden. PCR-analysis of a pooled faecal sample of all animals, and 
subsequent examination of individual faeces, confirmed the excretion of MAP by two rock hyraxes. In contrast, MAP was not detected in the faeces of 20 non-human primates (NHP), belonging to seven different species, although the presence of MAP was confirmed in the ileum of one cottontop tamarin (Saguinus oedipus), and the bone marrow of a common marmoset (Callithrix jacchus). Analogous to the examination of the NHP, the distribution of MAP within the body of the host was also investigated in the bull and the rock hyraxes. MAP manifestation within most body regions of clinically asymptomatic ruminants as well as non-ruminants was observed, including in the tissues of the gastrointestinal tract, urogenital tract, cardiovascular system, and/or respiratory system. Since none of the investigated animals (the bull; the group of rock hyraxes; or either of the non-human primate species) showed typical paratuberculosis clinical symptoms or lesions after necropsy, an asymptomatic infection with MAP was assumed. Therefore, it can be expected that MAP replicates within the asymptomatically infected host without triggering typical pathohistological changes or clinical signs of paratuberculosis. Furthermore, it is reasonable to suspect that nonruminants excrete the pathogen intermittently and in different concentrations, as clinically asymptomatic ruminants do.

In order to control a disease, it is necessary to discover all routes of transmission. Although since 1970 it has been known that MAP is present in the semen and the reproductive organs of bulls with clinical symptoms and/or paratuberculosis-typical pathological changes, only a small number of studies have scrutinised the role of bulls in the transmission of MAP. In this thesis, the presence of MAP was confirmed in the semen and reproductive organs of an asymptomatic infected bull. These results support the hypotheses that MAP might be transmitted to cows via semen under natural mating conditions, or, likely, by artificial insemination; however, the influence of the semen extender on the viability of MAP is unknown. In addition to the investigated bull, MAP was also observed in the reproductive organs of one female and one male rock hyrax. Interestingly, female animals of this species have a haemoendothelial placenta, meaning the foetuses have direct contact with the maternal blood, and that vertical transmission of MAP might also, therefore, be a possibility in non-ruminants.

Another interesting finding of this thesis, was the detection of MAP in the bone marrow of the investigated bull and the common marmoset. As it has been demonstrated that a MAP-related pathogen, Mycobacterium tuberculosis, can persist within the 
mesenchyme cells of the bone marrow in a viable, but not culturable, stage, it was concluded that the bone marrow might be a niche for the establishment of latent tuberculosis infection. Therefore, it might also be assumed that bone marrow plays a key role in the establishment of latent paratuberculosis infection.

Currently, available detection methods (PCR, cultivation, ELISA) require sophisticated laboratory equipment; to allow the identification of MAP-infected animals in lowresource settings or directly in the stable, a simple molecular diagnostic tool is needed. In order to fill this gap, an RPA assay targeting the MAP IS900 gene was developed. In contrast to commonly used diagnostic methods (culture and real-time PCR), RPA assays are extremely fast (3-15 minutes), easy to perform, run at a constant temperature of $42^{\circ} \mathrm{C}$, and all reagents are cold-chain independent. The developed MAP-RPA assay has a limit of detection of 16 DNA molecules, and does not show any cross-reactivity to other bacterial strains of clinical importance. The clinical specificity and sensitivity is calculated at $100 \%(\mathrm{n}=20)$ and $90 \%(\mathrm{n}=48)$, respectively, based on comparison with a well-established real-time PCR.

In conclusion, the studies performed for this thesis highlight the potential risk of MAP transmission from asymptomatic animals. Ruminants as well as non-ruminants play an important role in the silent spread of MAP within an animal stock. The obtained results confirm the high occurrence rate of MAP in semen and the male reproductive organs. Thus, bulls might be a part of the transmission cycle of MAP to cows and their descendants or foetuses. Furthermore, non-ruminants seem to be more susceptible to MAP than commonly believed. For the first time, the presence of MAP in rock hyraxes and the common marmoset was confirmed. Further studies should address the pathogenesis of clinically asymptomatic infected ruminants and non-ruminants, and routes of transmission via semen as well as between different species. 
GENERAL INTRODUCTION

1 GENERAL INTRODUCTION 
GENERAL INTRODUCTION

MYCOBACTERIUM AVIUM SUBSP. PARATUBERCULOSIS (MAP)

\section{TAXONOMY}

The genus Mycobacterium and the closely related Corynebacterium, Nocardia and Rhodococcus are members of the order Actinomycetales. Mycobacterium belongs to the Mycobacteriaceae family (Table 1). Although MAP was once considered a separate species, current taxonomy ranks MAP to the Mycobacterium avium-complex (MAC) (Turenne and Alexander, 2010).

Table 1: $\quad$ Taxonomy of MAP (NCBI Taxonomy Browser, 2017)

\begin{tabular}{ll}
\hline & ORGANISM TAXONOMY \\
\hline Kingdom & Bacteria \\
Phylum & Actinobacteria \\
Class & Actinobacteria \\
Order & Corynebacteriales \\
Family & Mycobacteriaceae \\
Genus & Mycobacterium \\
Species & Mycobacterium avium \\
\hline
\end{tabular}

The genus Mycobacterium consists of three major groups: Mycobacterium tuberculosis complex, Mycobacterium leprae, and all other non-tuberculosis mycobacteria, also called 'Mycobacteria other than tuberculosis' (MOTT). Currently, over 150 MOTTs have been officially described (Tortoli, 2014). Traditionally, the classification schema for MOTT has been based on phenotypic characteristics. MOTTs were divided into four Runyon Groups (I-IV) on the basis of features such as growth rate, growth temperature, pigmentation and biochemical activities (Rolle and Mayr, 2002). Mycobacterium kansasii, Mycobacterium marinum, Mycobacterium asiaticum and Mycobacterium simiae are some agents found in Runyon Group I. Characteristics of Runyon Group I mycobacteria are their slow growth rate and distinctive pigmentation (bright yellow in white light). Mycobacteria belonging to Runyon Group II also have a slow growth rate, but are pigmented yellow in white light and orange in the dark. Agents of this group 
include Mycobacterium scrofulaceum, Mycobacterium gordonae, Mycobacterium szulgae, Mycobacterium xenopi, and Mycobacterium flavescens. Slowly growing, photochromogenic mycobacteria belong to Runyon Group III. This group includes Mycobacterium ulcerans, Mycobacterium haemophilum and Mycobacterium malmoense. Although some strains of the Mycobacterium avium complex (MAC) have bright yellow pigmentation, they also belong to Runyon Group III. MAC species include Mycobacterium intracellulare, Mycobacterium avium subsp. avium (MAA), Mycobacterium avium subsp. hominissuis (MAH), Mycobacterium avium subsp. silvaticum (MAS) and Mycobacterium avium subsp. paratuberculosis (MAP). All fastgrowing mycobacteria (within seven days) belong to Runyon Group IV; this group includes species such as Mycobacterium fortuitum, Mycobacterium abcessus and Mycobacterium chelonae.

To date, MAC species have been characterised on the basis of phenotypic features and genetic analyses. Phylogenetic trees of mycobacteria were mostly designed on the basis of complete 16S rRNA and/or of amino acid sequences by sequencing DNA fragments of the hsp65 and rpoB genes. Currently, ten species of slow-growing mycobacteria and a subset of "MAC-other" isolates with an undetermined classification belong to the MAC. These closely related mycobacteria are distinguished by host preference, degrees of pathogenicity and environmental distribution (Table 2). 


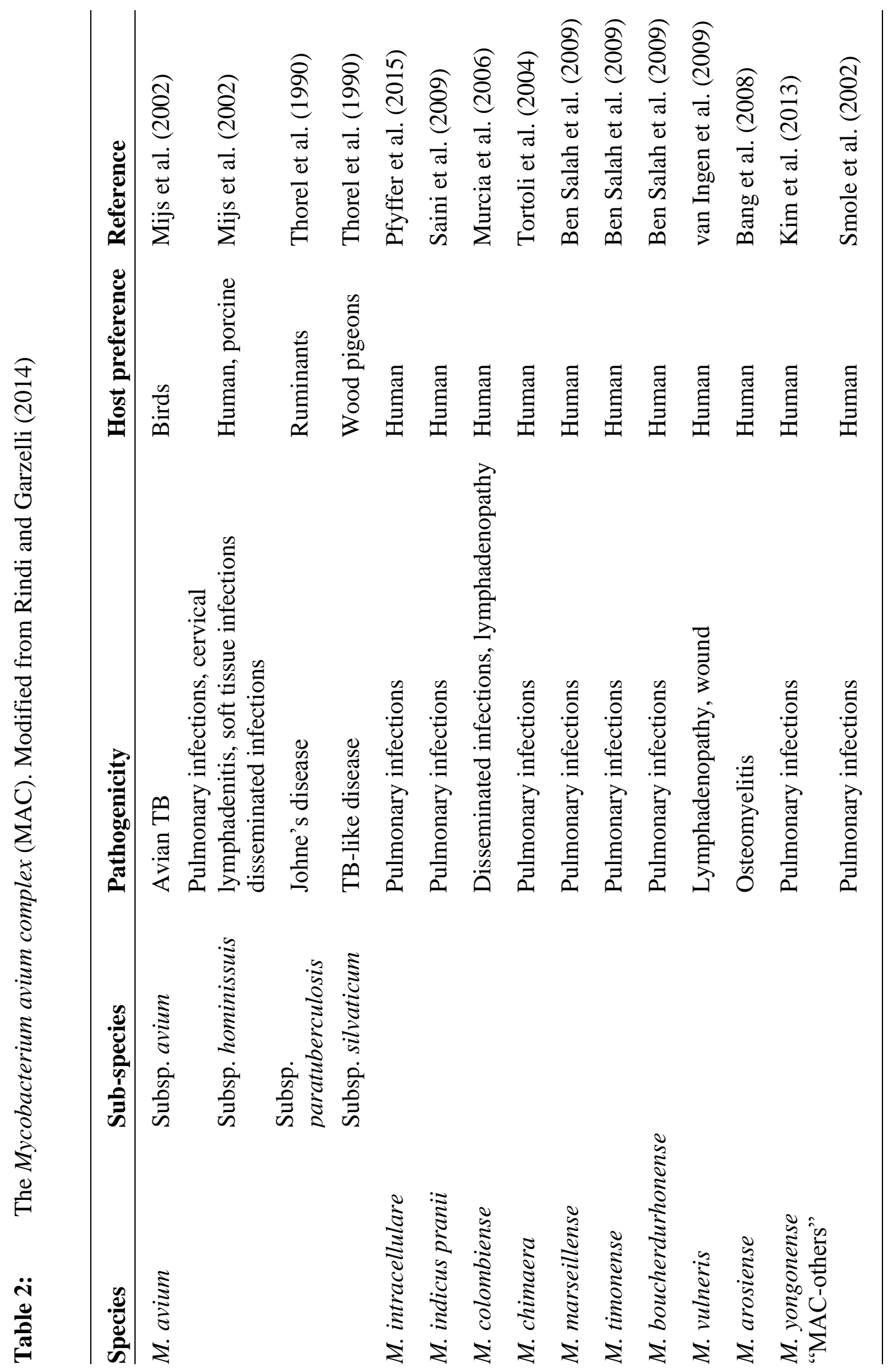


Currently, four subspecies belong to $M$. avium, including $M$. avium subsp. avium (MAH), M. avium subsp. paratuberculosis (MAP), M. avium subsp. silvaticum (MAS) and M. avium subsp. hominissuis (MAH). Although these subspecies were taxonomically closely related mycobacteria, each has a specific pathogenicity and host range (Rindi and Garzelli, 2014). Recently, availability of complete genome sequences and the presence of different insertion sequences (IS) among the M. avium subspecies have provided the opportunity to differentiate these subspecies by molecular biological typing methods. The distinction between the M. avium subspecies is largely based on IS1245, IS1311, IS900 and IS901 (Rindi and Garzelli, 2014).

\section{PATHOGEN CHARACTERIZATION AND TENACITY}

MAP is an acid-fast, non-moving, Gram-positive rod that is $0.5-2.0 \mu \mathrm{m}$ long and 0.3-0.5 $\mu \mathrm{m}$ wide (Manning and Collins, 2001). This pathogen grows under aerobic conditions and has a generation interval of 1.3-4.4 days (Lambrecht et al., 1988). A further characteristic of MAP is its mycobactin-dependent growth, which must be considered during bacteriological cultivation and is used as an exclusion criterion in the differentiation from other mycobacteria.

Free, long-chain fatty acids in the cell wall make the bacterium resistant to acid and alcohol. The cell wall structure is divided into three different layers (Daffe and Draper, 1998). The most outer part is a mycolic acid-containing layer with a membrane-like structure underneath lies the murein layer followed by the inner-most plasma membrane. Through the use of intercellular filaments, MAP is able to form bacterial nests, known as clusters (Merkal, 1973).

Due to this special cell wall structure, MAP is able to survive for long durations in the environment. MAP can survive for up to 270 days in the faeces of cattle (Larsen et al., 1956). On contaminated pastures, the pathogen is able to survive for up to 11 months (Gay and Sherman, 1992). Very sensitive MAP responds only to UV radiation (Whittington et al., 2004). The pathogen can thus survive on grass-covered pastures. Here, it is protected against UV radiation for up to 32 weeks; on completely bare, grazed sheep pastures for up to two weeks. Another influence on the survival of MAP is soil pH (Ward and Perez, 2004; Whittington et al., 2004). 
MAP also has a very high thermal stability. MAP can survive at both very low and very high temperatures. Cultivation of MAP from the naturally contaminated faeces of cattle remains possible after storage for 12 months at $-14{ }^{\circ} \mathrm{C}$ (Larsen et al., 1956). Its particularly high heat stability is especially important for food production. It has been demonstrated that viable MAP cells remain detectable in pasteurized milk (Grant et al., 1998). The pathogen is also not completely killed during the production of cheese (Spahr and Schafroth, 2001).

Due to this high durability, the range of effective disinfectants is limited. Faeces and dirt often surround the pathogen; therefore, most common disinfectants are ineffective. To date, only formaldehyde-containing disinfectants (Chiodini et al., 1984a) and peracetic acid agents (chemical sterilization) have been proven effective.

\section{GENOTYPES}

On the base of different molecular techniques, diverse strain types of MAP have previously been detected. Collins et al. (1990) proved that two major groups of MAP strains exist, which were named after the first known host species. The "Type $S$ " strains (sheep-type) were detected in sheep. This strain is characterized by an extremely slow growth (up to 12 months), and isolates are usually pigmented. In contrast, isolates belonging to the "Type C" strains (cattle-type) show growth within 5-16 weeks, and are unpigmented. Isolates of this type were often observed in cattle, but were also detected in a wide range of other non-ruminant animals (Ghadiali et al., 2004; Hutchings et al., 2010; Motiwala et al., 2004). Until recently, it was thought that a third group of MAP strains existed, the termed "Type III" or "intermediate" strains. It was expected that "Type III" strains were intermediate strains between the Type S and Type C strains; however, whole sequence data of these isolates has demonstrated that no Type $S$ strains exist, but that such isolates are actually a subtype of the Type S strains (Stevenson, 2015). Another group of MAP strains are the "Bison" or "B-type" strains. This strain type was differentiated by IS1311 PCR-REA (restriction enzyme analysis) on the basis of the number of IS1311 copies with C/T polymorphisms at position 223. Furthermore, B-type isolates from India are distinguishable from American isolates by a TG deletion at positions 64 and 65 of the IS1331 at locus 2 (Sohal et al., 2013). For this reason, these strains are grouped into the "Indian Bison Type," which is a sub-lineage of Type C strains (Thibault et al., 2012). 


\section{GENOME}

In 2005, the first whole genome sequence (WGS) of a MAP isolate (MAP strain K10; GenBank No. AE016958) was published. The analysis performed of the Type II strain yielded a single circular sequence of $4,829,781$ base pairs, with a $\mathrm{G}+\mathrm{C}$ content of $69.3 \%$ (Li et al., 2005). After optical mapping and resequencing of the MAP strain K10 genome by the Illumina sequencing platform, an edited sequence and annotation was issued. To date, the WGS of five different MAP isolates have been published. These isolates originated from the USA, Egypt and India, and were isolated from cattle as well as from one human breast milk sample. WGS data demonstrates that the genome size of MAP ranges from $4.78 \mathrm{Mb}$ to $4.83 \mathrm{Mb}$. The $\mathrm{G}+\mathrm{C}$ content of all five isolates is $69.30 \%$. The number of genes ranges from 4,446 to 4,464, and the number of proteins ranges from 4,153 to 4,321. Furthermore, sequence data of a further 34 MAP isolates are presently housed in the NCBI databank (Table 3). These isolates come from varying hosts, such as cattle, sheep, goat, camel, human and red deer. Moreover, these isolates were isolated in different countries, including for example the USA, India and China. 


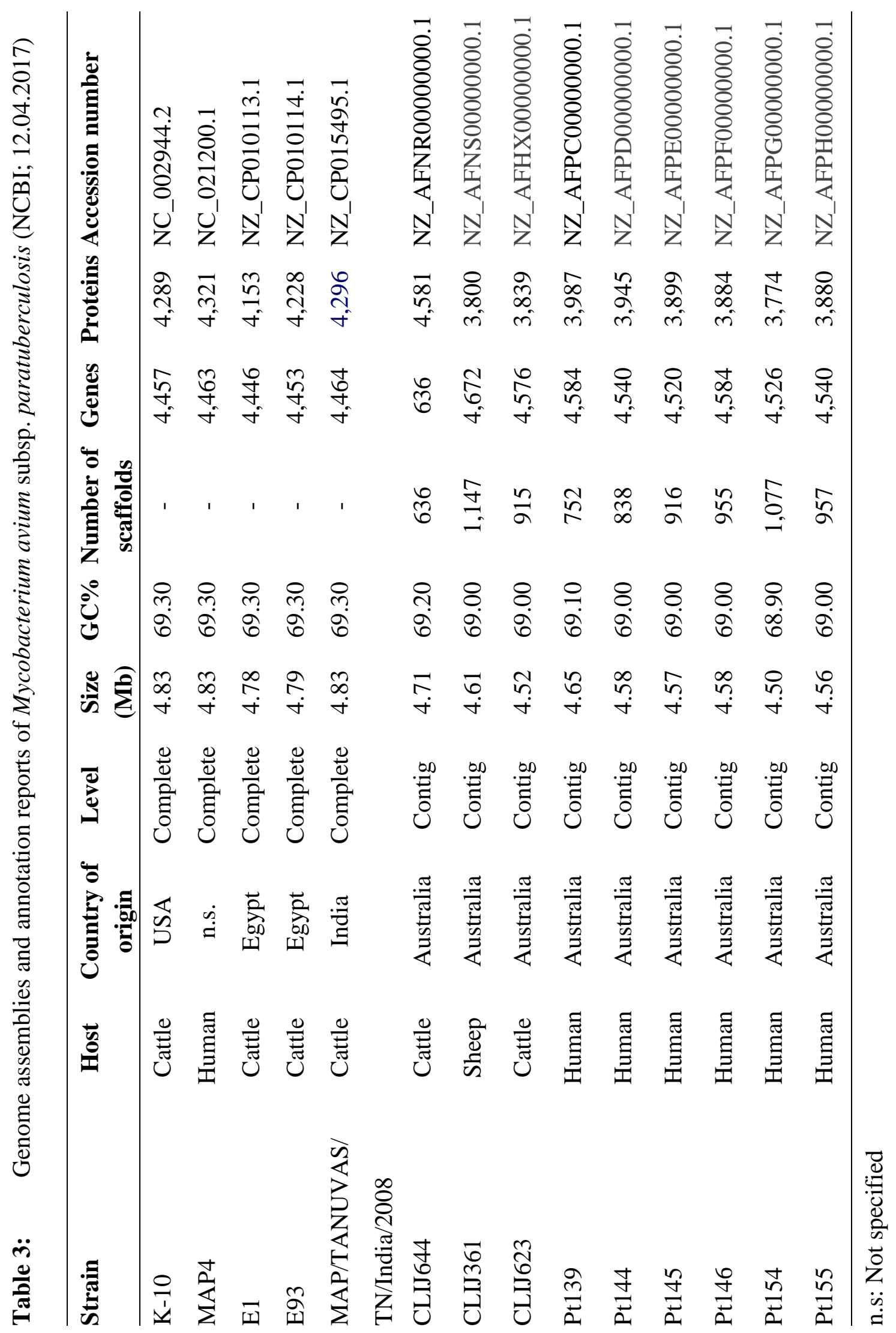




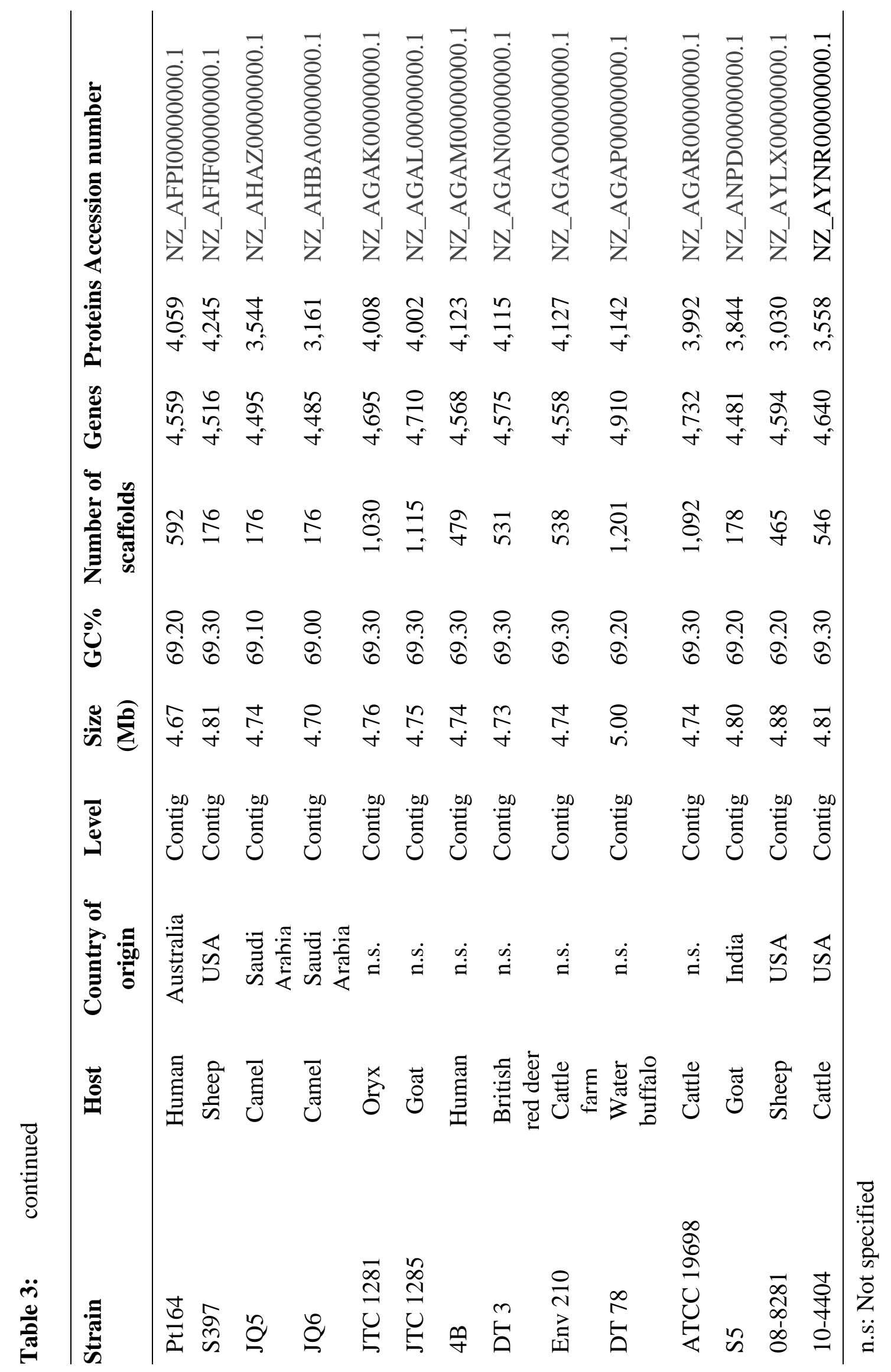




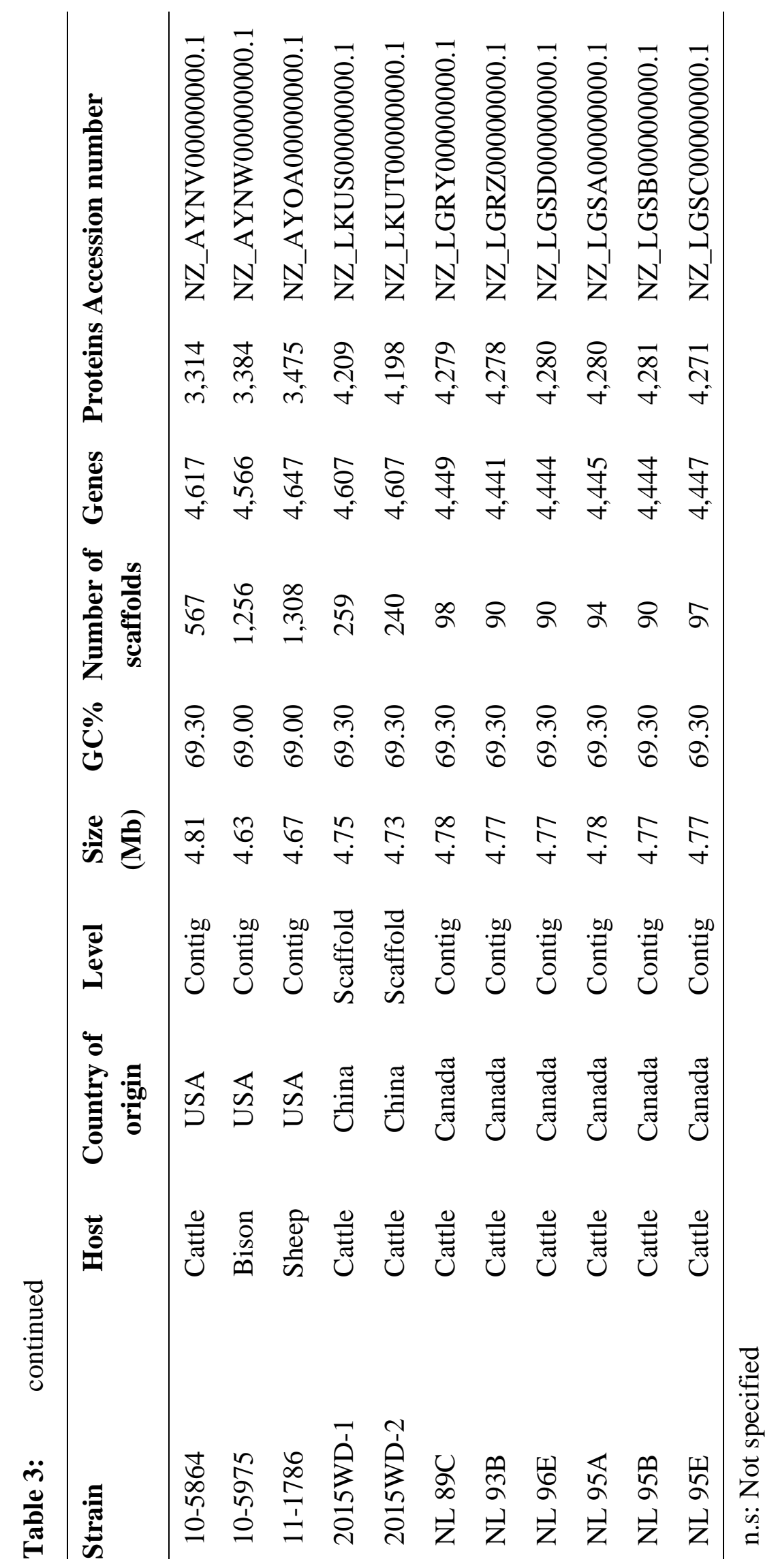




\section{PARATUBERCULOSIS}

Mycobacterium avium ssp. paratuberculosis (MAP) is the infectious agent of Johne's disease. The disease is characterized by a degenerative chronic granulomatous inflammation of the intestinal tract, causing diarrhoea, weight loss, reduced reproductive performance, and eventually death (Harris and Barletta, 2001). In general, an infection with MAP occurs in domestic ruminants; however, the pathogen has also been detected in a wide range of other domesticated, wild, and zoo animals, including non-ruminants (Beard et al., 2001a; Greig et al., 1999; Münster et al., 2013a). In accordance with the Ordinance on Notifiable Animal Diseases (Anonymous, 2011), paratuberculosis is a notifiable disease in Germany. Clinical cases of the disease are recorded via the Tierseuchen Nachrichten System (Animal Disease News System).

\section{HISTORY OF PARATUBERCULOSIS}

Initial reports of the occurrence of paratuberculosis were reported in the mid-1820s, but the cases were not well described and the cause was unknown. The initial clear description of a paratuberculosis case was reported in 1895 by Dr. Heinrich Albert Johne and Dr. Landedon Fortingham (Johne and Fortingham, 1895). The authors observed thickened intestinal mucosa and enlarged mesenteric lymph nodes during the necropsy of a cow from Oldenburg (Germany). In the inflamed tissue, numerous of acid-fast (red-staining) bacteria were found. As the tuberculosis-like bacterium did not elicit disease in guinea pigs and failed to grow in standard conditions used for Mycobacterium tuberculosis, it was concluded that the detected pathogen was the same as that which caused tuberculosis in birds. The clinical picture was coined pseudotuberculosis enteritis due to similarities in pathological changes, such as intestinal tuberculosis.

After the first report of pseudotuberculosis enteritis, several reports of the new disease began to be recounted from Europa and the USA. In 1906, Professor Bernhardt Bang discovered that cattle that became sick with the new disease did not show an immune response to Mycobacterium bovis tuberculin antigens, whereas Mycobacterium avium tuberculin did trigger an immune reaction (Bang, 1906). The first cultivation of the pathogen causing this new disease occurred by way of a laboratory accident. Contamination with Mycobacterium phlei provided essential nutrients for the growth of 
the then-named Mycobacterium enteritidis chronicae pseudotuberculosae bovis (Twort and Ingram, 1912). Now that cultivation of the pathogen was possible, diagnostic tests were able to be established. As had been done for tuberculosis, a so-called 'johnin tuberculin,' to be used in skin testing, was developed. Furthermore, complement fixation and agglutination techniques evolved to detect an antibody reaction in serum samples.

In 1920, cases of paratuberculosis also began to be reported in Africa and Asian. From this moment, it become obvious that paratuberculosis had spread worldwide. The first official name of the aetiological agent was Mycobacterium paratuberculosis, which was published in Bergey's Manual of Determinative Bacteriology in 1923. Since 1940, it has been known that paratuberculosis is not only a disease of domesticated livestock; rather, wildlife can also be affected.

It must be noted that data published between 1920 and the 1970s was performed with the working strain ATCC 12227, also called strain 18. Today, it is known that stain 18 was a laboratory contamination and not a Mycobacterium paratuberculosis, but rather a strain of Mycobacterium avium serovar 2 (Chiodini, 2005).

The first time the pathogen was isolated from a human was in 1984 from a patient with Crohn's disease. Although it was long assumed that a link between Mycobacterium paratuberculosis and Crohn's disease exists, this was the first physical evidence of the link. Essential for the moleculobiological detection of the pathogen was the detection of the insertion element (IS) 900 by two independent research groups (Green et al., 1989; McFadden et al., 1987). After several name changes, the pathogen was renamed, based on DNA-DNA hybridization analyses (Thorel et al., 1990), to Mycobacterium avium subsp. paratuberculosis (MAP).

\section{PATHOGENESIS}

The pathogenesis of paratuberculosis remains incompletely understood. Immune status, age, the pathogen concentration ingested and the resistance profile of the affected animal all play important roles (Dirksen et al., 2003) in the course of the infection. Whitlock and Buergelt (1996) divided the disease course of paratuberculosis into four stages: 
STAGE I: "Silent” infection of calves, young livestock and adults

STAGE II: Subclinical disease of carrier adults

STAGE III: Clinical disease

STAGE IV: Advanced clinical disease.

STAGE I: "Silent" infection of calves, young livestock and adults

Calves and young animals are generally infected via the faecal-oral route, through the ingestion of faeces or milk/colostrum contaminated with MAP. After oral ingestion, the pathogens enter the digestive tract and are transported by M-cells into the intestinal epithelium (Buergelt, 1991; Momotani et al., 1988). After the manifestation of MAP in the intestinal epithelium, the pathogen is transported to the lymphoid tissue (Peyer's patches) of the intestine, to the mesentery and via the thoracic duct into other organs (Chiodini and Rossiter, 1996). The subepithelial and intraepithelial macrophages are the target cells for MAP. A cell-mediated immune response of the host is encouraged (Chiodini et al., 1984a; Clarke, 1997). However, the pathogen is able to survive these cellular defence mechanisms.

STAGE II: Subclinical disease of carrier adults

At this stage, MAP commences intracellular replication within the macrophages. The pathogen is then either excreted in the faeces or phagocytosed by other macrophages. With pathogen release, the cellular immune response changes into the humoral immune response (Chiodini et al., 1984a). Pathomorphology shows low-to-moderate mucosal hypertrophy and the noticeable enlargement of mesenteric lymph nodes. At this stage, the infected animal shows no clinical symptoms. 
STAGE III: Clinical disease

Infected animals first develop clinical symptoms at an age of between two and five years (Larsen et al., 1975; Valentin-Weigand and Goethe, 1999). During this stage, the feed intake and general condition of the animal initially remains undisturbed (Rosenberger, 1978). Weight loss, diarrhoea, and a decrease in milk production and fattening performance are the first signs of the disease observed. Humoral immunity predominates during this stage of the disease (Chiodini et al., 1984a), and the detection of antibodies is possible.

STAGE IV: Advanced clinical disease

In the final stage, diseased animals show massive clinical signs. These animals suffer extreme weight loss, despite undisturbed feed intake (Figure 1). Another feature is recurring watery, foul-smelling diarrhoea. With disease progression, general health is increasingly disturbed (Dirksen et al., 2003). Oedema, cachexia and serous atrophy of body fat occur in severe cases (Clarke, 1997; Olsen et al., 2002). Pathologically, it is noticeable that the mucosa of the ileum, jejunum and colon has a high degree of hypertrophy (Figure 2). The mesenteric lymph nodes are also severely enlarged. In the final stage, the humoral immune response decreases to anergy (Chiodini, 1996). 


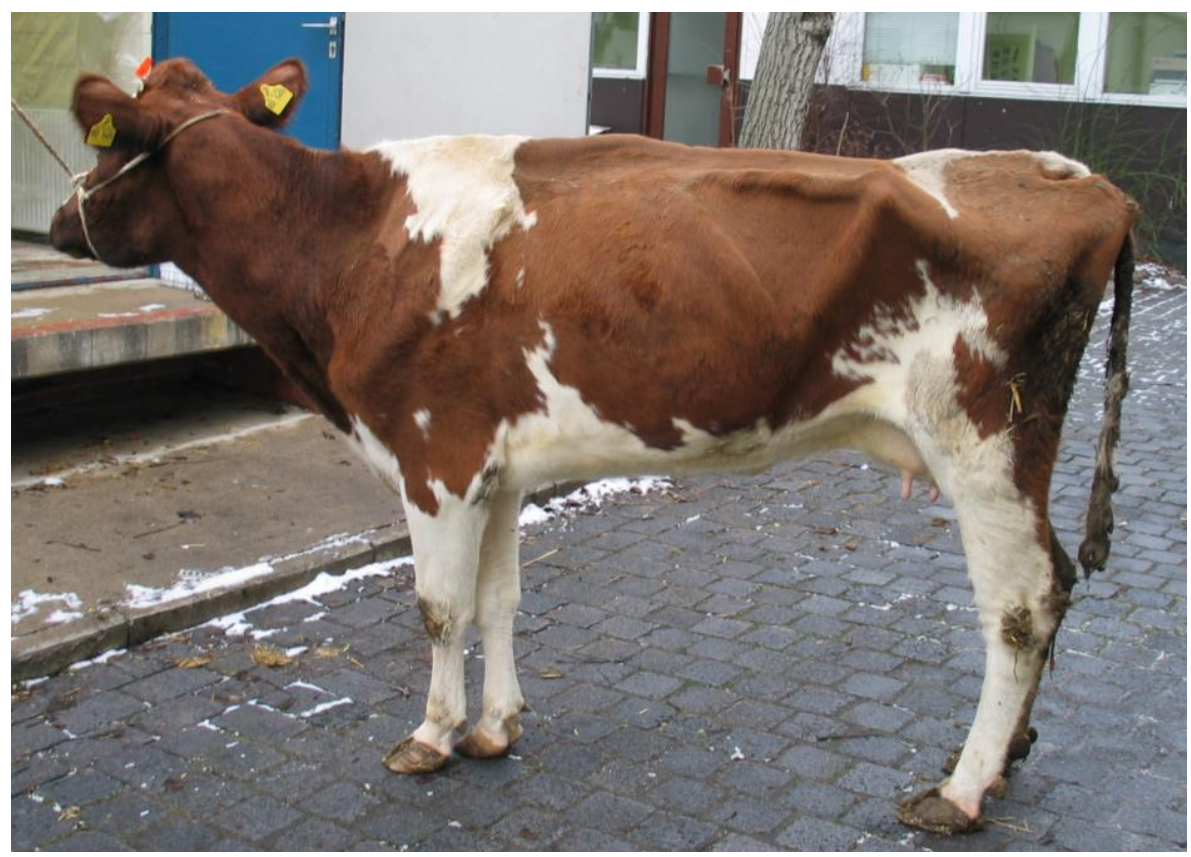

Figure 1: Dairy cattle in the final stage of paratuberculosis (Division of Microbiology and Animal Hygiene, Georg-August-University Göttingen)

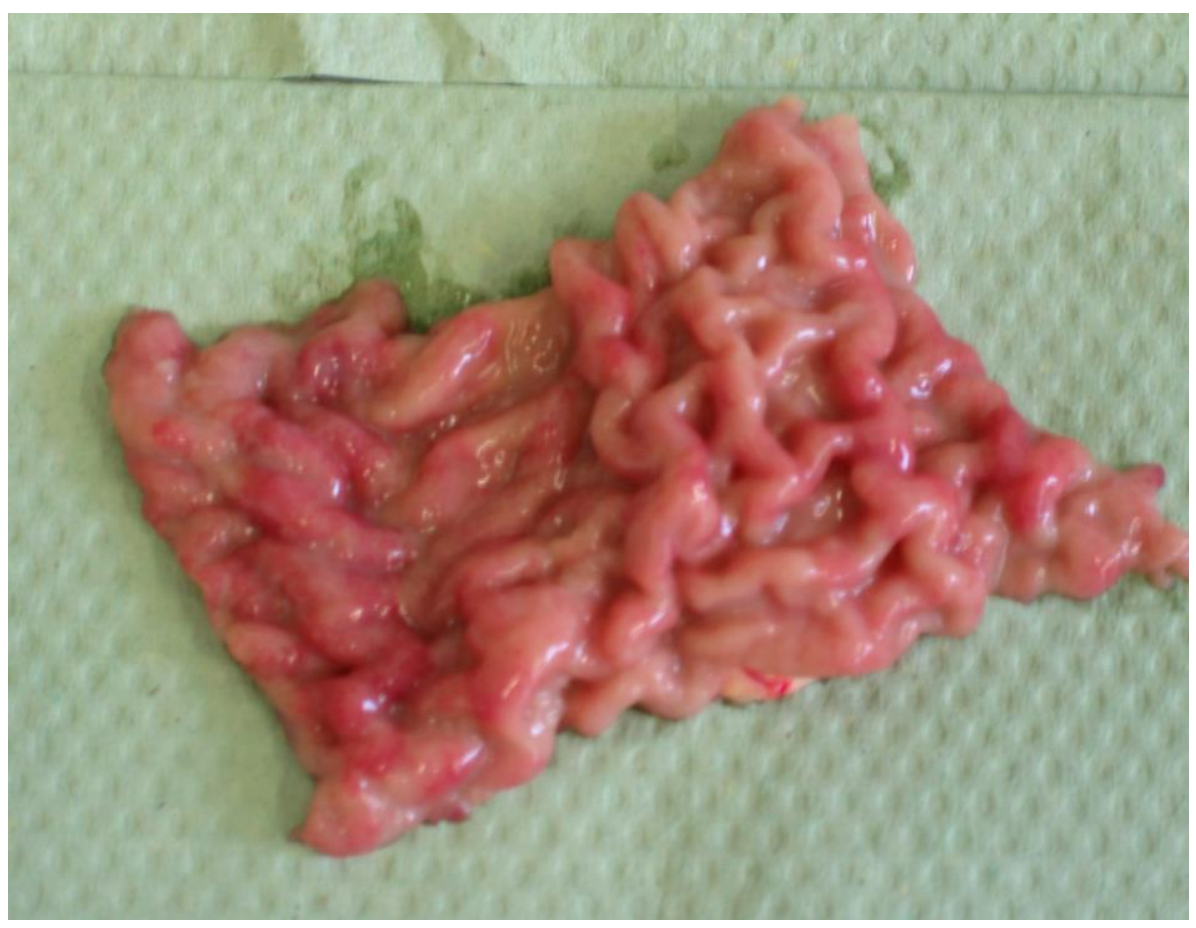

Figure 2: $\quad$ Pathological findings of paratuberculosis (Division of Microbiology and Animal Hygiene, Georg-August- University Göttingen) 
Primary MAP is present in the tissues of the gastrointestinal tract and appending lymph nodes (Sweeney et al., 1992b). In a study with tissue samples from 131 cattle collected in a slaughterhouse, MAP was detected most frequently (77.6\%) in the tissues of the gastrointestinal tract (Hasonova et al., 2009). In addition, many studies have reported the occurrence of MAP in tissues other than gastrointestinal tract (Table 4) or reproductive system of cows and heifers (Table 5) and bulls (Table 6). It is likely that MAP is disseminated within the host in macrophages via the blood or lymphatic system (Clarke, 1997). It was originally thought that the spread of MAP within the host occurs during the advance disease stage (Chiodini, 1996); however, other studies have reported that the pathogen is spared as early as the subclinical infection stage (Buergelt et al., 1978; Condron et al., 1994). A recent study concluded that the dissemination of MAP within the host take place soon after infection (Mortier et al., 2013).

Table 4: Distribution of MAP in extraintestinal tissues other than the reproductive tract of infected cattle

\begin{tabular}{ll}
\hline Specimens & References \\
\hline Liver and hepatic lnn. & Antognoli et al. (2008); Collins (1997); Hasonova et al. \\
& (2009); Khol et al. (2010); Pavlik et al. (2000) \\
Retropharyngeal lnn. & Pavlik et al. (2000) \\
Mandibular lnn. & Pavlik et al. (2000) \\
Spleen & Collins (1997); Hasonova et al. (2009); Pavlik et al. \\
& (2000) \\
Lung and lnn. & Antognoli et al. (2008); Collins (1997); Hasonova et al. \\
Kidneys & (2009);Hasonova et al. (2009); Pavlik et al. (2000) \\
Popliteal lnn. & Antognoli et al. (2008); Collins (1997) \\
Prescapular lnn. & Antognoli et al. (2008) \\
Heart & Antognoli et al. (2008) \\
Blood & Antognoli et al. (2008) \\
& Collins (1997); Buergelt and Williams (2004); Buergelt \\
& et al. (2004); Juste et al. (2005); Khol et al. (2010); \\
& Münster et al. (2013b)
\end{tabular}

lnn: Lymph nodes 
Table 5: Distribution of MAP in the reproductive system of cows and heifers

\begin{tabular}{ll}
\hline Specimens & References \\
\hline Udder & Collins (1997); Doyle (1954); Hasonova et al. (2009); \\
& Merkal (1984) \\
Supramammary lnn. & Alexejeff-Goleff (1929); Antognoli et al. (2008); \\
& Hasonova et al. (2009); Pavlik et al. (2000); (Sweeney \\
& et al., 1992a) \\
& Doyle (1954); Buergelt and Williams (2004); Smith \\
Milk & (1960); Sweeney et al. (1992b); Taylor et al. (1981) \\
& Collins (1997); Hasonova et al. (2009) \\
Uterus & Pearson and McClelland (1955); Merkal (1984); Seitz \\
Foetus & et al. (1989); Sweeney et al. (1992b) \\
Foetal membranes & Doyle (1958) \\
Cotyledons & Doyle (1958); Lawrence (1956); Pearson and \\
& McClelland (1955); \\
Uterine flush & Pribylova et al. (2013); Rohde and Shulaw (1990) \\
Vaginal flush & Pribylova et al. (2013) \\
Follicular fluid & Pribylova et al. (2013) \\
\hline
\end{tabular}

lnn: Lymph nodes 
Table 6: $\quad$ Distribution of MAP in the reproductive system of bulls

\begin{tabular}{ll}
\hline Specimens & References \\
\hline Testes & Ayele et al. (2004); Glawischnig et al. (2004); Tunkle \\
& and Aleraj (1965) \\
Bulbourethral gland & Larsen and Kopecky (1970); Tunkle and Aleraj (1965) \\
Epididymis & Ayele et al. (2004); Collins (1997); Khol et al. (2010) \\
Seminal vesicle & Ayele et al. (2004); Khol et al. (2010); Larsen and \\
& Kopecky (1970); Larsen et al. (1981) \\
Prostate gland & Larsen and Kopecky (1970); Larsen et al. (1981) \\
Semen & Ayele et al. (2004); Buergelt et al. (2004); Eppleston \\
& and Whittington (2001); Khamesipour et al. (2014); \\
& Khol et al. (2010); Larsen and Kopecky (1970); Larsen \\
& et al. (1981); Münster et al. (2013b); Sharifzadeh et al. \\
& (2010); Tunkle and Aleraj (1965) \\
\hline
\end{tabular}

\section{SUSCEPTIBILITY AND TRANSMISSION}

Calves younger than 30 days appear to be the most susceptible to a MAP infection (Clarke, 1997; Larsen and Kopecky, 1970). Neonates are considered to be at especially high risk of becoming infected with MAP. The cause of the increased susceptibility of newborn animals is currently unknown; however, as Peyer's patches have been observed to be the major point of entry for MAP (Momotani et al., 1988; Sigurdardottir et al., 2005), it is assumed that the peak of activity of Peyer's patches seen in neonates may play an important role in their susceptibility. Cattle over two years of age are considered to be resistant to infection; however, an infection with MAP can occur at all ages.

An infection with MAP does not only depend on age. Other factors, such as infectious dose of MAP, pathogen virulence, stress factors or the genetic profile of the host also have an impact on infection susceptibility and the disease course. It is assumed that an infection with MAP occurs in approximately $85 \%$ of the subsequently infected animals within the first weeks of life, $10 \%$ of the animals are infected in utero and only $5 \%$ of the animals are infected within the first year of life. To trigger an infection, a minimum 
infectious dose of $10^{3}$ pathogens per calf is required (Gerlach, 2002). In experimental studies, a relationship between the infectious dose and the development of lesions and clinical signs has been reported (Begg and Whittington, 2008). Furthermore, it was observed that earlier disease development occurs after larger doses of MAP. Furthermore, stress factors such as intensive livestock production, poor feeding, transport and calving may also influence the disease course (Chiodini et al., 1984a). Differences between breeds in the incidence of infection with MAP and disease exist. The clinical picture of paratuberculosis appears to occur more frequently in Jersey or Guernsey cattle, in comparison to other breeds (Cetinkaya et al., 1997; Jakobsen et al., 2000). In the literature, the heritability $\left(\mathrm{h}^{2}\right)$ of an infection with MAP in dairy cattle has been estimated to range from 0.09-0.12 (Gonda et al., 2006; Hinger et al., 2008; Koets et al., 2000; Mortensen et al., 2004). Several candidate genes that were considered to have an impact on host susceptibility to MAP in cattle have also been studied.

MAP can be transmitted vertically and horizontally to susceptible hosts. The theoretical pathways of MAP transmission are illustrated in Figure 3. In the literature, three different routes of infection with MAP are described. The main infectious route is the oral route. Although MAP is excreted through the faeces, milk and semen by infected animals (Sweeney, 1996), MAP faecal-contaminated colostrum, milk, or feed represents the primary sources of infection for susceptible hosts. Furthermore, MAP is also widespread in environmental samples of beef and/or dairy farms, such as in water (Norby et al., 2007; Raizman et al., 2004), slurry (Gwozdz, 2006; Jorgensen, 1977), sewage (Gwozdz, 2006) and sediment (Whittington et al., 2005). MAP is commonly found in environmental samples from the parlour exits, floors of holding pens, common alleyways, lagoons, manure spreaders and manure pits (Lombard et al., 2006; Raizman et al., 2004). The grass and soil of pastures can also be contaminated with MAP by the faecal excretion of grazing animals (Fecteau et al., 2010). 
GENERAL INTRODUCTION

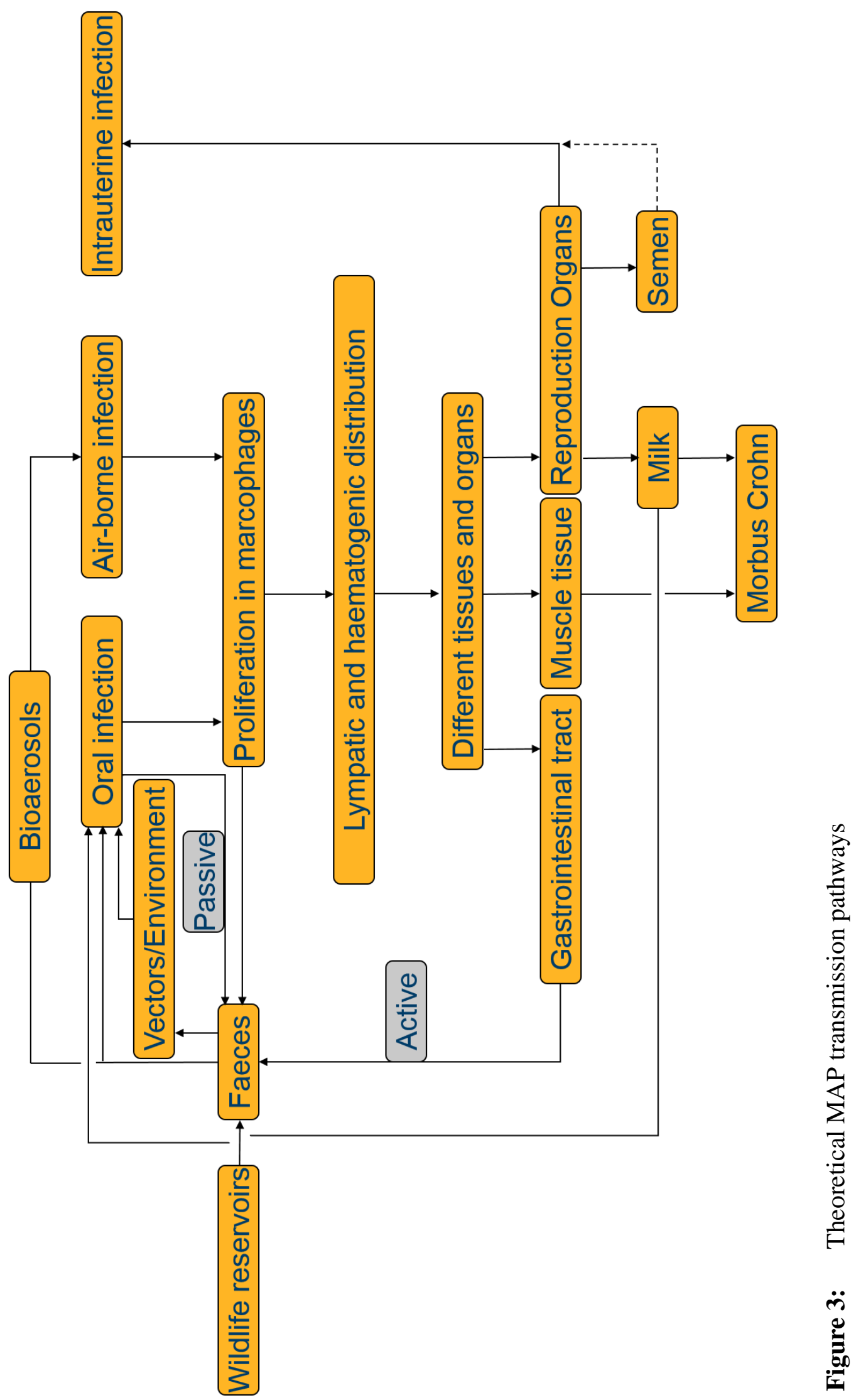


The biggest problem for the control of MAP spread are the subclinically infected adult MAP shedders. These animals show no clinical signs, but excrete the pathogen intermittently in infectious concentrations (Münster et al., 2013b). In general, infected adult animals can be divided into three categories based on the degree of MAP shedding via the faeces (Raizman et al., 2007; Whitlock et al., 2000):

Low shedders: $\quad<10 \mathrm{CFU} /$ tube

Moderate shedders: $10-49 \mathrm{CFU} /$ tube

Heavy shedders: $\quad>50 \mathrm{CFU} /$ tube.

Calves can also excrete MAP in their faeces; however, this potential source of infection is often underestimated because many studies focus on the examination of adult animals alone. Furthermore, it is difficult to identify infected calves because MAP is only excreted intermittently and in amounts below the limits of detection of the most-used diagnostic tool. In a recent study, the presence of MAP was demonstrated in the faeces of a naturally infected five-month-old calf (Hasonova et al., 2009). It has also been demonstrated in a longitudinal study of experimentally infected calves that a peak of MAP shedding occurs shortly after MAP inoculation and again two months after inoculation (Mortier et al., 2014).

Other sources of infection are milk and colostrum from subclinically and clinically infected cows. In the literature, pathogen concentrations of 2-8 CFU per $50 \mathrm{ml}$ milk were reported in the milk of infected dairy cattle (Sweeney et al., 1992a). However, the mechanism of MAP extraction into milk remains unclear. An explanation for this may be that MAP is directly excreted into milk from lymphatics draining into the mammary glands. Indirect faecal contamination of the milk during the milking process is also likely (Hasonova et al., 2009).

MAP is systemically spread in infected hosts. Therefore, prenatal transmission of the pathogen from clinically and subclinically infected cows to their foetuses is common and well recognized (Seitz et al., 1989; Sweeney et al., 1992b). A metal-analysis estimated that the prevalence of prenatal infection is $9 \%$ (95\% confidence interval [CI]: 6-14\%) for subclinically diseased cows and 39\% (95\% CI: 20-60\%) for clinically diseased cows (Whittington and Windsor, 2009). However, it is currently unknown 
whether such intrauterine infected animals more rapidly develop clinical disease and what their shedding or antibody response pattern is.

Transmission of MAP via semen may be possible; however, this route of transmission is unlikely as in most previous studies only low concentrations of MAP were measurable in the ejaculate from infected bulls (Buergelt et al., 2004; Philpott, 1993). Furthermore, the transmission of MAP via the semen to a healthy cow has never been reported. However, Merkal et al. (1982) demonstrated that MAP can be found in cow uterine fluids and uterine walls after artificial contamination of the uterus. A longitudinal study of a subclinically infected bull showed that MAP is repeatedly detectable in semen in concentrations of up to $10^{5} \mathrm{MAP}$ genome equivalent/ml semen. MAP has also been found in the male reproductive organs (Table 6). A prevalence study showed that $20.0 \%$ of the breeding (47/235) and $33.3 \%$ of the teaser bulls (6/18) semen production units in Punjab (Pakistan) had a serological antibody response against MAP (Abbas et al., 2011). It has also been known for over 45 years that MAP could survive the antibiotic treatment and freezing procedure of yore used in artificial insemination (Larsen and Kopecky, 1970). Therefore, bulls should be considered a potential source of transmission, especially due to their direct and indirect (artificial insemination) contact with many cows (Khol et al., 2010).

Another source of infection for ruminates appears to be MAP-containing dust. Under experimental and natural conditions, the presence of MAP in settled dust collected from dairy farms was confirmed (Eisenberg et al., 2010a; Eisenberg et al., 2010b). Furthermore, recent experimental studies have demonstrated that an infection with MAP in cattle as well as in sheep could occur by the inhalation of MAP through the nasal and transtracheal routes. An infection via both routes can trigger a manifestation of MAP in the intestinal tract and corresponding lymph nodes.

The introduction of MAP into former paratuberculosis-free herds most likely occurs by the purchase of diagnostically and clinically unrecognized infected animals (Sweeney, 1996). Another source of MAP introduction may be manure movement between farms, the purchase of colostrum or milk and the sharing of pastures or water between farms (Lombard, 2011). In addition to the transmission between domestic ruminants, wild ruminants, and a wide range of non-ruminants can also be infected with MAP. Wild ruminant species such as the white-tailed deer (Odocoileus virginianus) (Chiodini and Van Kruiningen, 1983), red deer (Cervus elaphus), roe deer (Capreolus capreolus) 
(Nebbia et al., 2000; Robino et al., 2008) and fallow deer (Cervus dama dama) (Balseiro et al., 2008) seem to be true MAP reservoirs (Carta et al., 2013). In contrast to such diseased ruminant species, infected non-ruminants usually do not develop macroscopic or microscopic lesions. The only well-studied non-ruminant species are wild rabbits. These animals can excrete MAP in concentrations of up to $7.6 \times 10^{5}$ CFU/g in faeces (Daniels et al., 2003). Furthermore, MAP has also been detected in the urine of MAP-infected rabbits (Daniels et al., 2003). It was recently demonstrated that the high prevalence of MAP in rabbits is associated with difficulties in controlling paratuberculosis in cattle farms in East Scotland (Shaughnessy et al., 2013). Compared with this knowledge about rabbits, only a few case reports on other clinically diseased non-ruminant species have been described in the literature. One example of this is a MAP-infected miniature donkey (Equus asinus $f$. asinus) with typical clinical and pathomorphological changes (Stief et al., 2012). Transmission between wild and domestic animals most likely occurs via the intake of MAP faecal-contaminated material due to the shared use of the same environment, such as pasture area (Carta et al., 2013).

\section{PREVALENCE}

Paratuberculosis is distributed worldwide (Turenne and Alexander, 2010) (Figure 4). The World Organisation for Animal Health (OIE) classifies paratuberculosis as a disease with socioeconomic importance and significance for human health. Several studies have revealed a herd level prevalence of 6-55\% in Europe (Boelaert et al., 2000; Gasteiner et al., 1999; Manning and Collins, 2001); however, only a few studies have provided comparable and reliable estimates of the prevalence of MAP infection in different ruminants. The following grounds are the main reasons for the lack of comparable prevalence: i) Varying definitions of the condition "paratuberculosis," ii) difficulties in detecting the condition using the currently available diagnostic options, and iii) deficient study designs. Based on these aspects, a review analysing the published herd and animal prevalence of MAP infection in farmed animals in Europe was highly critical of the findings. A proper conclusion regarding the prevalence of MAP infections in Europe cannot be made. Estimates of the true prevalence among cattle are approximately $20 \%$, and herd level prevalence is likely to be $>50 \%$ in many countries (Nielsen and Toft, 2009). 


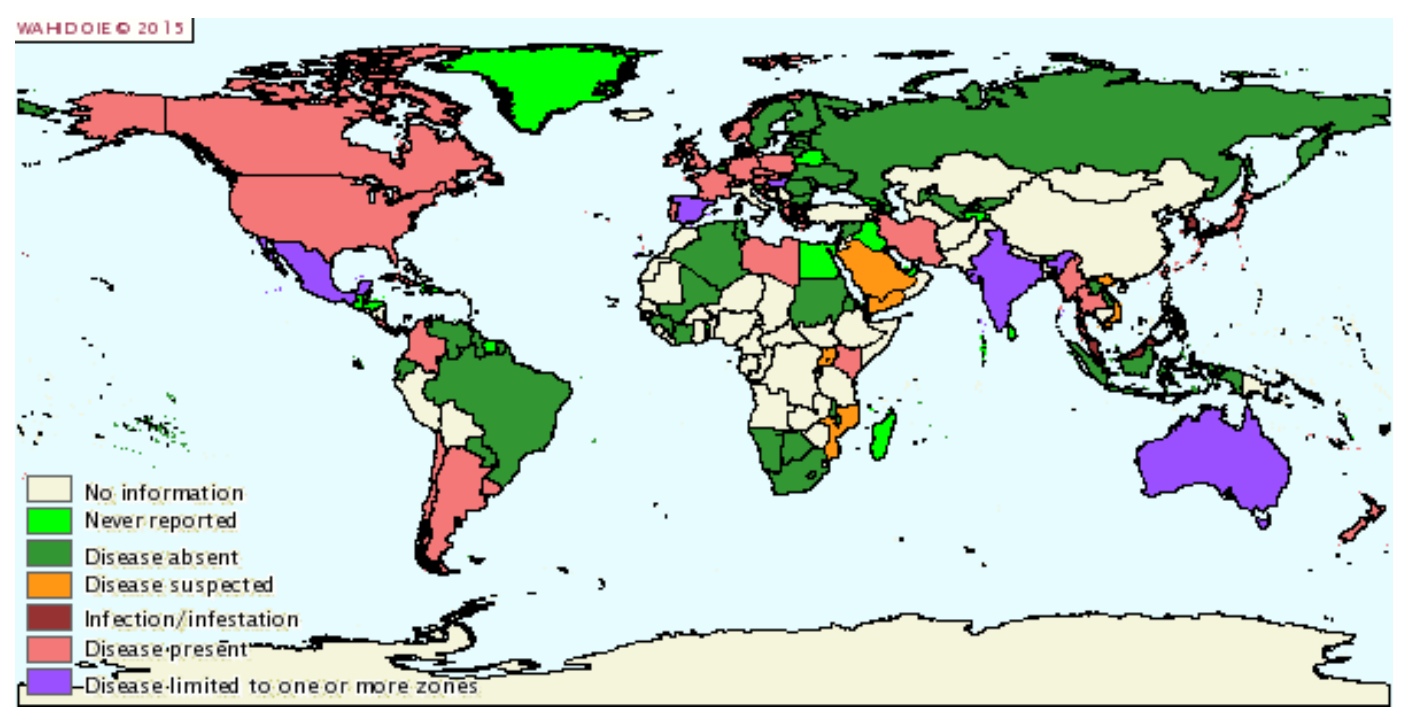

Figure 4: $\quad$ Worldwide presence of paratuberculosis in 2015 (OIE, 06.11.2015)

Paratuberculosis is also widespread throughout Germany, and is a notifiable disease. In Germany, all confirmed cases of paratuberculosis are documented in the animal disease intelligence database. In 2014, the number of confirmed paratuberculosis cases had increasing to 502 cases per year. In Figure 5, the reported cases of paratuberculosis between 1995 and 2014 are listed in detail. In Lower Saxony, a voluntary control program was introduced in 1990. Paratuberculosis control programs have also been established in other federal states, such as North Rhine-Westphalia and Thuringia. No comprehensive, nationwide studies on the prevalence of paratuberculosis have been performed. Therefore, an estimation of disease prevalence in Germany is currently not possible. 


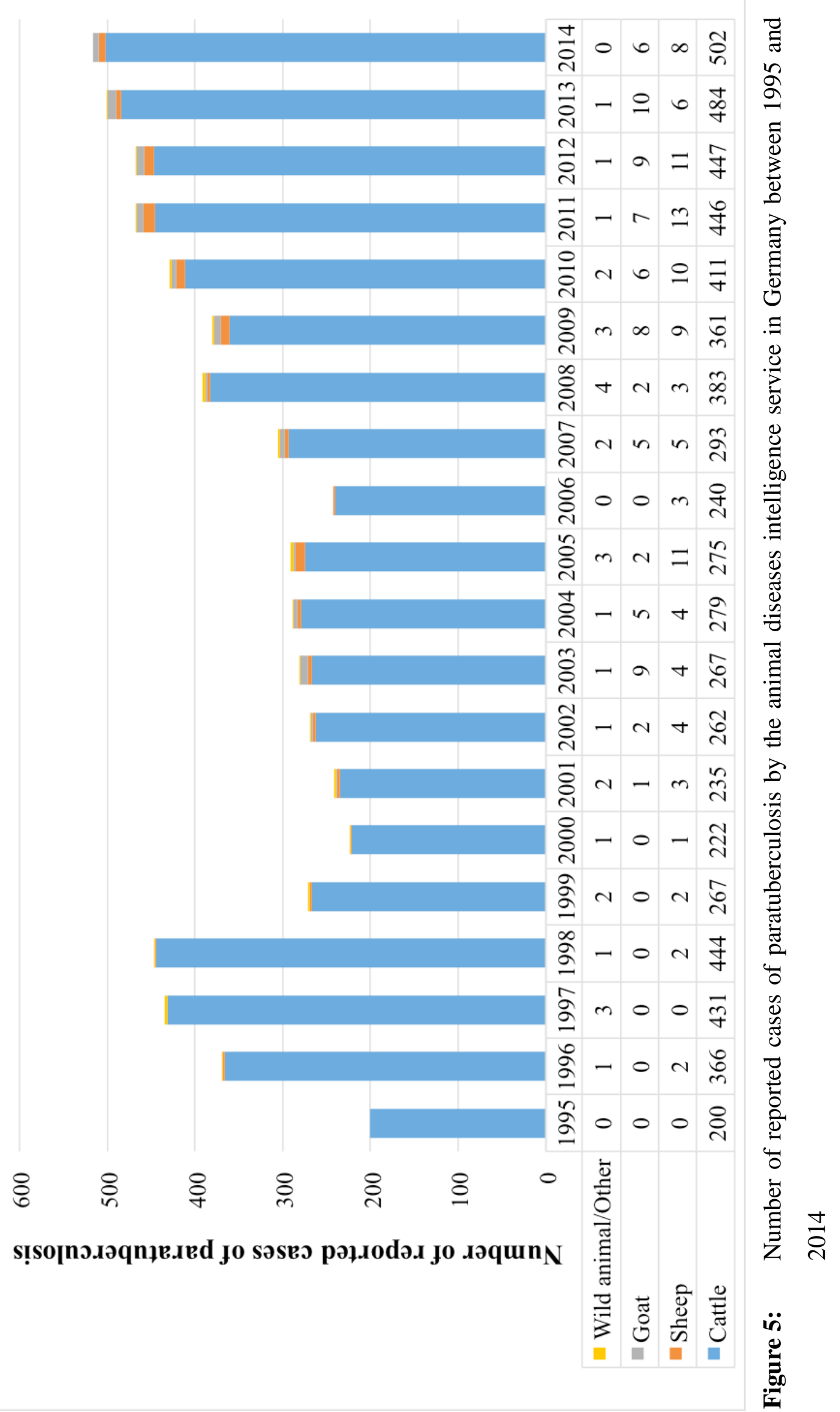




\section{HOST RANGE}

In principle, paratuberculosis is a diarrhoeal disease of domestic ruminants. Bovine, ovine and caprine animals are particularly affected. The causative agent is also found in a large number of cervids and other zoo and wild ruminants, such as bison, antelope, camelid, zebu, yak, and water buffalo (Chiodini et al., 1984a; Deutz et al., 2005; Godfroid et al., 2005; Manning et al., 2003; Ridge et al., 1995; Sivakumar et al., 2005; Zavgorodniy et al., 2015). The typical symptoms of the disease can also develop in some wild and zoo ruminants (Figure 6). Infected wild animals likely play an important role in the spread of MAP, forming a potential source of infection for domestic ruminants (Chiodini and Van Kruiningen, 1983).

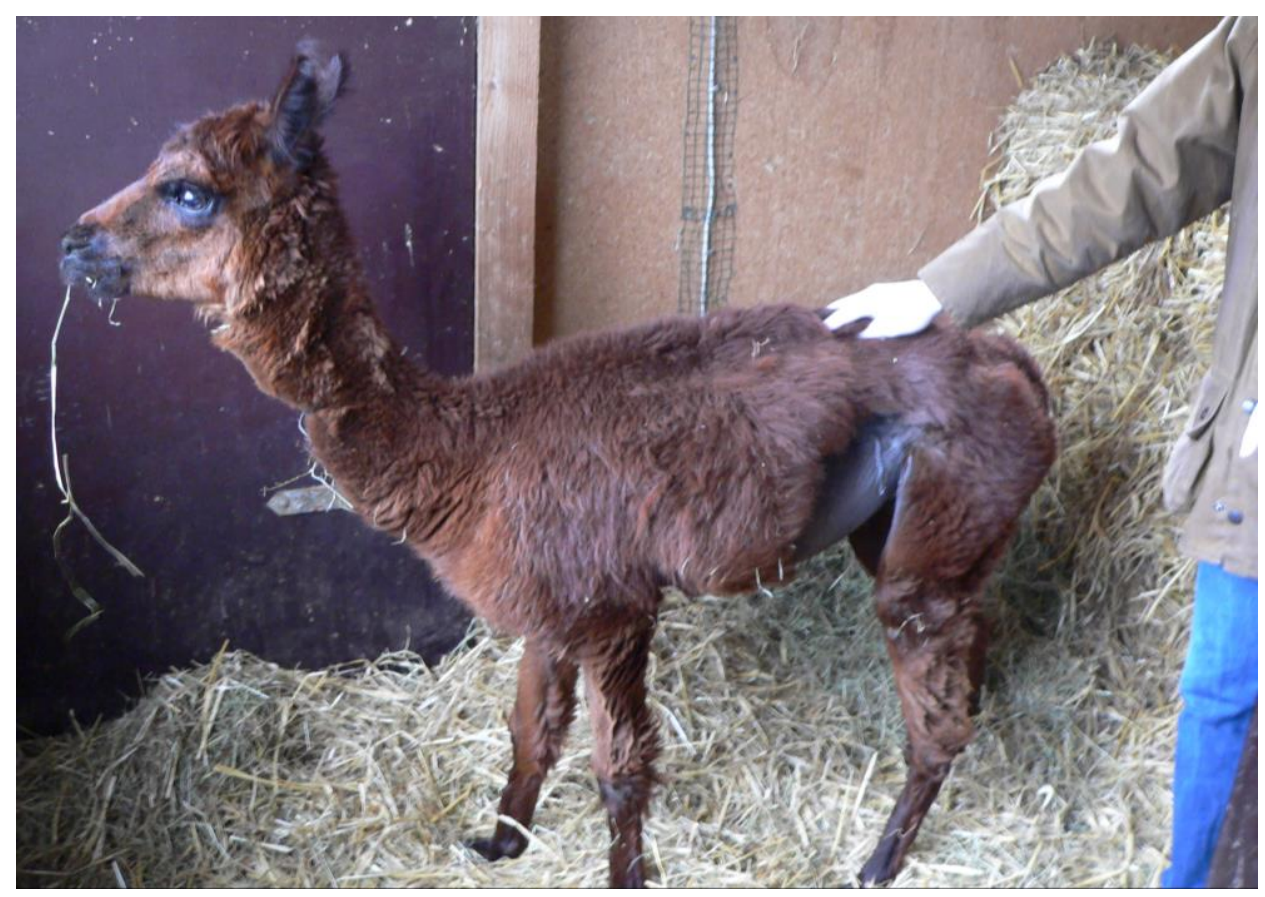

Figure 6: Alpaca in the final stage of paratuberculosis (veterinarian Aloys von Buchholz, Rheine)

The pathogen has also been detected in monogastric animals. In most cases, these infected animals do not develop clinical symptoms (Chiodini et al., 1984a). Monogastric animals, in which the pathogen can be isolated, include for example nonhuman primates (McClure et al., 1987; Singh et al., 2011; Zwick et al., 2002), foxes (Beard et al., 1999), snow bunnies (Deutz et al., 2005), raccoons and banded animals (Corn et al., 2005). In the case of wild rabbits, the pathogen has been isolated from faeces and pathological changes were found during autopsy that were very similar to 
those expected in paratuberculosis (Greig et al., 1997). McClure et al. (1987) performed investigations in a stump-tail macaque herd. Samples from a total of 38 animals were analysed using PCR. MAP was detected in 28 animals, and 13 of these animals died within five years. The deceased animals showed typical symptoms of paratuberculosis.

\section{ZOONOTIC ASPECT}

For the first time, Dalziel (1913) associated the chronic inflammation of the human intestine known as Crohn's disease (CD) with the intestinal infection of cattle known as paratuberculosis. Since that time, the relationship between MAP and CD has been debated. Nevertheless, the "Guidelines for the management of paratuberculosis in ruminants", published by the German Federal Ministry of Food, Agriculture and Consumer Protection (Anonymous, 2005), advised the potential risk for humans to get infected with MAP. MAP is widespread in the food chain. Humans may be exposed to MAP through the consumption of dairy and beef products such as meat (Alonso-Hearn et al., 2009), milk (Ayele et al., 2005; Ellingson et al., 2005; Grant et al., 2002) and cheese (Spahr and Schafroth, 2001).

MAP was initially isolated from three CD patients in 1984 (Chiodini et al., 1984b). Since then, the different matrices of CD patients have been tested for MAP in many other studies (Kirkwood et al., 2009; Schwartz et al., 2000). For instance, in one study, the intestinal mucosal tissue of Sardinian CD patients and non-diseased people, who served as controls, were investigated for the presence of MAP using PCR (Sechi et al., 2005). The pathogen was detected in 25 patients (83.3\%) with $\mathrm{CD}$ and three control patients $(10.3 \%)$. To confirm the PCR results, tissues were also prepared for culture. Tissue culture confirmed the presence of MAP in all PCR-positive cases. In another study, MAP DNA was detected via PCR in blood samples from 13 (46\%) patients with $\mathrm{CD}$, four (45\%) patients with ulcerative colitis (UC) and three (20\%) patients without inflammatory bowel disease. In that study, viable MAP was cultured from the blood of $14(50 \%)$ patients with CD and from two (22\%) patients with UC (Naser et al., 2004). Such studies indicate that MAP may be involved in the pathogenesis of CD. However, in other studies, MAP was not detected in the tissues of CD patients. For instance, MAP DNA was not present in intestinal biopsies of 81 Indian patients with CD (Sasikala et al., 2009). 
The aetiology of CD remains largely unknown. An infectious cause, a genetic predisposition, and an auto-immune-mediated phenomenon may all be involved in the pathogenesis of CD (Büttner et al., 2006).

\section{CONTROL MEASURES IN EUROPE}

The occurrence of paratuberculosis is not underestimated only in Germany. There are indications that the infection is endemic in most countries with a developed dairy industry (Kennedy and Benedictus, 2001) Similarly, a high prevalence is likely in most countries with cattle farming, although accurate estimations are not available (Nielsen and Toft, 2009). Control and monitoring programs are already underway in some countries (Bakker, 2010).

In the Netherlands, the aim of the program is not the certification of MAP-free herds, but the reduce of MAP concentration in milk. The Dutch Animal Health Service launched its quality assurance program in 2006. This program is based on the annual testing of milk samples from all dairy cattle and serum from all other cattle older than three years via ELISA. On the basis of the test results, the herds are classified into status A (negative test results), status B (positive result, corresponding animal was removed) or status $\mathrm{C}$ (positive result, corresponding animal remains in the herd) (Weber and Schaik, 2008). Since 2010, participation in the program has been mandatory. In order to ensure the quality of milk, since the first of January 2011 only the milk from herds with status A or B can be used by the dairy industry.

In Luxembourg, all animals older than 24 months have been tested using ELISA. The classification into different statuses is based on the percentage of test-positive animals within a herd. A herd with status A has 0\% ELISA-positive animals, status B has $<5 \%$, status C 5-10\%, and status D >10\% ELISA-positive animals. Herds without sampling or without removal of positive animals are marked as level O. Based on the status of a herd, different measurements are then recommended (Bakker, 2010).

In Denmark, a voluntary monitoring program has existed since 2006. It aims to provide tools for the farmer to control MAP infection and to reduce the prevalence within the country (Nielsen, 2007). Part of this program is the elevation of the paratuberculosis status based on the results of a three-monthly milk ELISA. In contrast to other programs, the herds are not classified into different categories. 
In Belgium, all animals older than 30 months are tested, via a milk ELISA for dairy cattle and serum ELISA for dry cattle. Cattle that test positive are slaughtered within six months. This voluntary control program has existed since 2008 .

In France, two national control programs exist: one to control herds that are infected with MAP and one to monitor MAP-free herds. The test regime depends on the status of the herd (one recently infected head of cattle or herds with endemic paratuberculosis). In herds with endemic paratuberculosis, faecal culture or PCR is recommended. Farmers are allowed to leave the program under certain conditions, for example if all tests have been negative in the previous two years. For the monitoring of MAP-free herds, animals that are older than 24 months are tested at an interval of between nine and 30 months by ELISA, faecal culture or PCR (Bakker, 2010).

In Austria, a compulsory control program for clinical paratuberculosis in sheep, goats and farmed deer has existed since 2006. In Italy and Spain, there is currently no nationwide established paratuberculosis control.

In Germany, no nationwide control program currently exists. Several federal states have established voluntary control programs, for example North Rhine-Westphalia (since 1998), Saxony (2003), Thuringia (2008), Hessen (2014), and Rhineland Palatinate. Guidelines for the control of paratuberculosis were published by the government in the "Recommendations of the Federal Ministry of Food and Agriculture on hygienic requirements for the keeping of ruminants" (Bätza, 2014). The aims of these guidelines are the standardisation of control measures in Germany, the prevention of MAP spread, the minimization of clinical cases and economical losses introduced by MAP, and prevalence reduction. On this basis, Thuringia published a new program for the control of paratuberculosis (Anonymous, 2015). The aims of this program are i) to reduce the prevalence of MAP infections in the herds, ii) contain spread to other cattle herds, and iii) the creation and protection of paratuberculosis-free stocks. In this program, three different phase of control were defined.

\section{Control phase}

This phase is divided into four different levels:

Level 1: For the evaluation of the herd status, semi-annual environmental faecal samples or pooled faecal samples are examined using bacteriological tests. 
Level 2: Heavy shedders (high risk animals) are identified annually by serological examination of all cattle older than 24 months. Animals testing positive should be slaughtered as soon as possible.

Level 3: For the identification of subclinically infected animals, faeces are annually examined using bacteriological methods. All animals that return a positive test should be slaughtered as soon as possible.

Level 4: In addition to all measures found in Level 3, cattle testing positive must be killed within one month after detection, and pregnant cattle must be killed within one month after calving.

2. Recognition and acquisition phase of the status "paratuberculosis unsuspected herd"

A herd is regarded as unsuspicious for paratuberculosis when the results of the examination of the recognition phase have been negative over a continuous period of three years, and when the obligatory hygienic measures have been completed. During the period of recognition, only cattle from herds of the same or higher phase and level of control should be purchased.

\section{Monitoring phase}

In order to maintain the status, cattle older than 30 months are examined using faecal testing at an interval of two years. In addition, environmental faecal samples are investigated for the presence of MAP on a semi-annual basis. Furthermore, animals with typical signs of disease are to be immediately examined using bacteriological methods. The obligatory hygienic measures must also be completed.

The hygienic measures of the program have the following objectives: i) Prevention of the new infection of young animals via the pathogen-free rearing of calves, ii) reduction in infection pressure by the early detection and removal of infected animals from the herd, and iii) prevention of disease introduction by the careful purchase and access of animals. 


\section{DIAGNOSTIC METHODS}

Ante-mortem diagnostic methods for MAP can be grouped into tests measuring the cellmediated or humoral immune response against MAP, and tests detecting the pathogen (Figure 7).

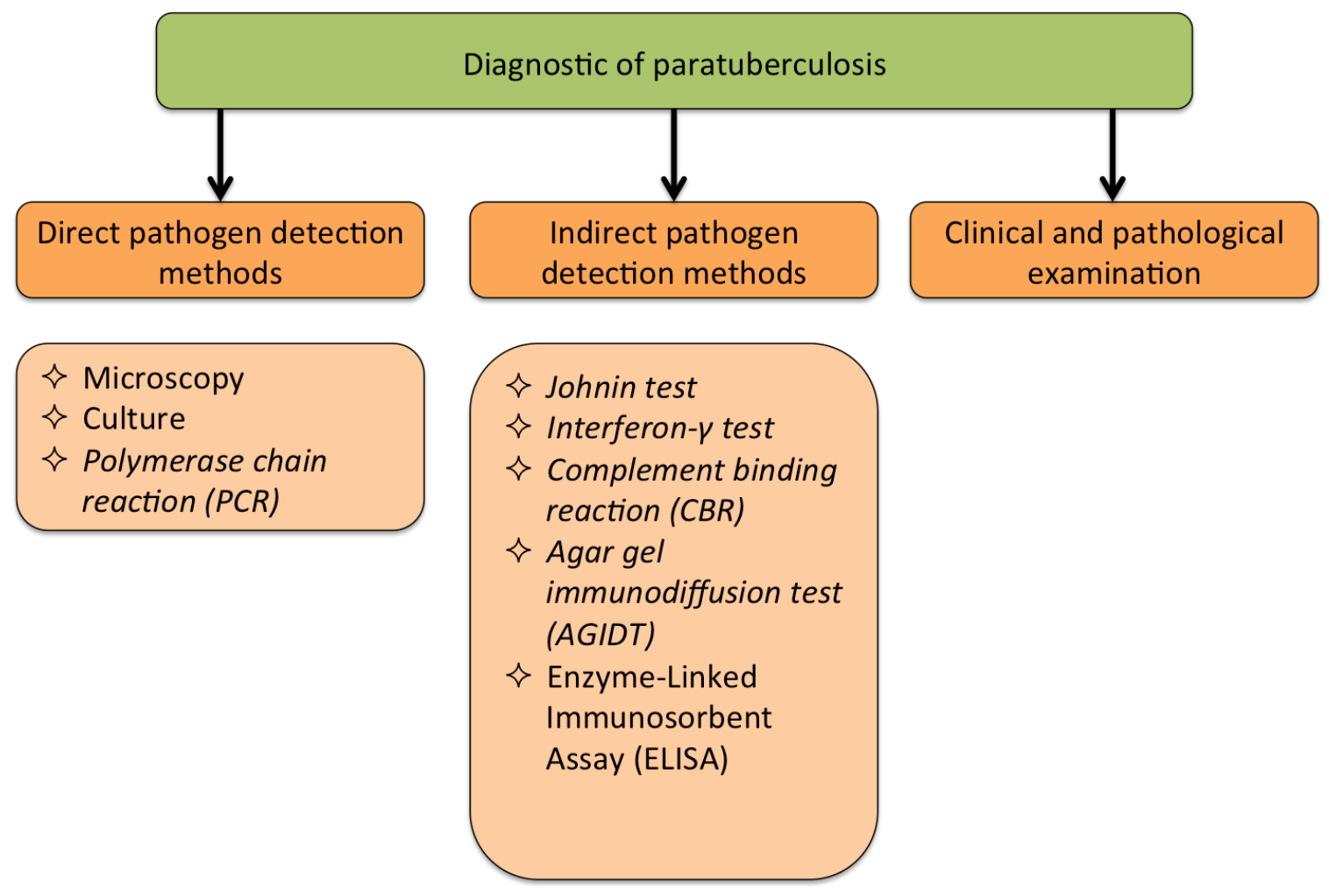

Figure 7: Diagnostic options to detect a MAP infection

Depending on the disease stage, the sensitivity and specificity of diagnostic tests vary (Nielsen and Toft, 2009). Due to the chronic character of paratuberculosis infection, the age of the animals influences the stage of the disease and hence the test response (Figure 8). 


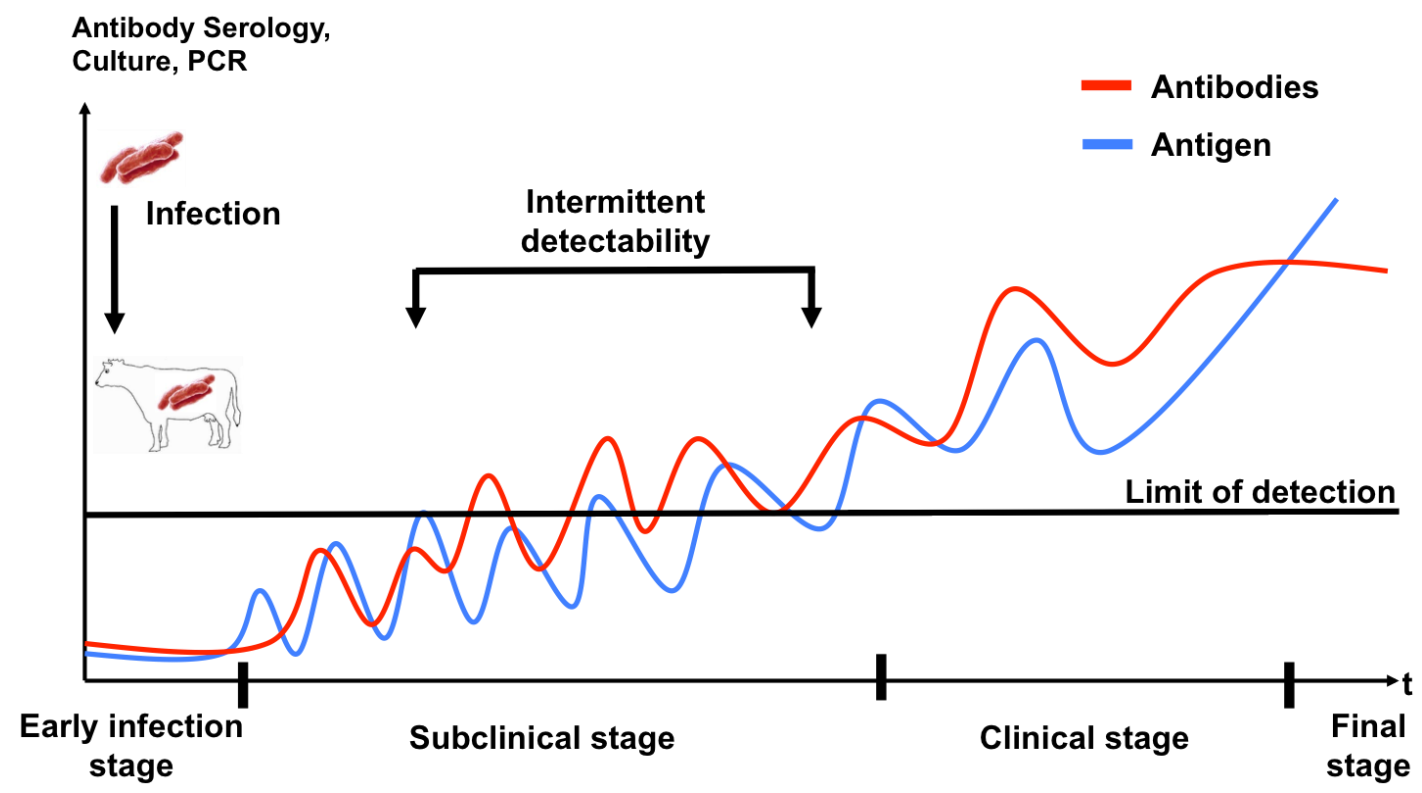

Figure 8: Detectability of the MAP agent or antibody response during different disease stages

\section{INDIRECT DIAGNOSTIC METHODS}

MAP-specific antibodies or a host MAP-specific cellular immune response are detected using indirect methods. A differentiation is here made between allergological (cellbased) and serological (antibody-based) methods.

\section{A) Johnin test}

The Johnin test is based on the cellular immune response, and belongs to the allergological test methods. For detection of the immune response, an avian tuberculin or Johnin/paratuberculin solution is administered to the animal either intradermally or intravenously. In the case of intradermal application, evaluation is made by measuring the thickness of the skin fold at the injection site. A differentiation is made between two reaction types (Merkal, 1973). The immediate type is an allergic reaction. This reaction is unspecific and manifests within two hours by an oedematous swelling at the injection site. Reactions occurring after 24 to 72 hours are the delayed reaction type. This results in a profound increase in wheal circumference at the injection site. In the intravenous application of the solution, the evaluation is based on the body temperature profile or the ratio of neutrophil granulocytes to lymphocytes (Chiodini et al., 1984a). However, it 
must be noted that cross-reactions with other mycobacteria can occur that distort the results (Clarke, 1997).

\section{B) Interferon- $\gamma$ test}

Another method based on the cellular (T cell-driven) immune response is the interferon$\gamma$ test. The mechanism of this test is that the T cells present in the blood are stimulated by mycobacterial antigens, producing interferon- $\gamma$. This cytokine is detectable using IFN- $\gamma$ ELISA (Stabel, 1996). An advantage of this method is that even young, infected animals with a low humoral immune response can be identified, and thus the test is also suitable for the detection of the disease in the early stages (Stabel et al., 2007). However, the sensitivity is limited to values between 13 and $85 \%$, and the specificity is moderate, with values between 66 and $67 \%$. Based on these parameters, the test is currently unsuitable for paratuberculosis diagnostics (Büttner et al., 2006).

\section{C) Complement-binding reaction (CBR)}

CBR was the first serological test designed for the detection of MAP antibodies in blood serum. The CBR is based on the detection of complement-binding antibodies in the serum of tested cattle. This test was previously obligatory for the international trade of cattle; however, the test lacks sensitivity, especially during the subclinical stage of disease. The possibility of cross-reactivity also had a limiting effect on specificity (Wilks et al., 1981). For these reasons, it is no longer used today (Sherman et al., 1990).

\section{D) Agar gel immunodiffusion test (AGIDT)}

The advantages of the AGIDT are that it is both cost-effective and fast. In this assay, MAP antigens are located in a gel. The tested serum is applied to the gel. Detection of MAP antibodies is via the antigen-antibody binding. The sensitivity is limited to values between 8.3 and 29.1\% (Ferreira et al., 2002; Gumber et al., 2006; Robbe-Austerman et al., 2006). The specificity of the method is between 92.5 and $99.5 \%$ (Ferreira et al., 2002; Robbe-Austerman et al., 2006). The AGIDT is particularly suitable for the rapid diagnosis of clinically ill animals, since these have a correspondingly high antibody level. Only at this stage does the test have a sufficient sensitivity (more than 50\%). However, even in clinically infected animals with progressive infection, often no antibodies are detected. 
E) Enzyme-Linked Immunosorbent Assay (ELISA)

The ELISA assays have the highest diagnostic value in the serological methods, since the antibody level has already increased prior to the occurrence of clinical signs. Although ELISA tests are currently well-suited to herd diagnostics, they have limitations in the diagnosis of individual animals. For paratuberculosis diagnostics, MAP-specific antigens are usually bound to a microtiter plate. Milk or serum can thus be tested for the presence of antibodies. When specific antibodies to MAP are present, an antigen-antibody complex is formed in the respective well. An enzyme-linked antiruminant immunoglobulin antibody is added to the complex, along with a chromogenic substrate, which then shows a photometrically measurable colour change.

The ELISA technique was initially in 1971 (Engvall and Perlmann). Seven years later, this method was used for the diagnosis of paratuberculosis (Berg and Jensen, 1978). Thereafter, further ELISA tests were developed with different but a significantly higher sensitivity and specificity than in CBR and AGIDT (Reichel et al., 1999). At present, a total of four manufacturers in Germany offer certified ELISA assays for the diagnosis of paratuberculosis. For the coating of the microtiter plates, for example, protoplasmic antigens or cell wall components are used in these ELISA methods. Studies have shown that the specificity is less pronounced without pre-absorption using Mycobacterium phlei. Cross-reactivity is significantly reduced by sera pre-absorption (Köhler et al., 2007). Furthermore, the sensitivity of the respective ELISA method depends on the stage of the infection. In subclinically infected animals, lower diagnostic sensitivities are generally found than in animals in the advanced disease stage. The diagnostic sensitivity of the tests in weak shedders is between 15.0 and $26.0 \%$, and in strong shedders between 75.9 and 88.1\% (Dargatz et al., 2001; Köhler et al., 2007; Sweeney et al., 2006; Whitlock et al., 2000).

\section{DIRECT DIAGNOSTIC METHODS}

In the case of direct pathogen detection, the pathogen or its DNA is detected.

\section{A) Microscopy}

The direct microscopic detection of pathogens by Ziehl-Neelsen staining is a costeffective and simple method to carry out (Figure 9). Ziehl-Neelsen staining is based on 
the acid and alcohol stability of MAP and can be derived from many different matrices, for example faeces, rectal mucosa or mesenteric lymph nodes. Due to the particular cell wall properties of MAP, the red dye carbol fuchsin cannot be removed by treatment with hydrochloric alcohol. Using the light microscope, MAP bacteria are marked as redcoloured, slender rods, which form clusters via intracellular filaments (Merkal, 1973). However, caution must be taken when interpreting the results, as this method has a low analytical sensitivity and specificity (Benazzi et al., 1996).

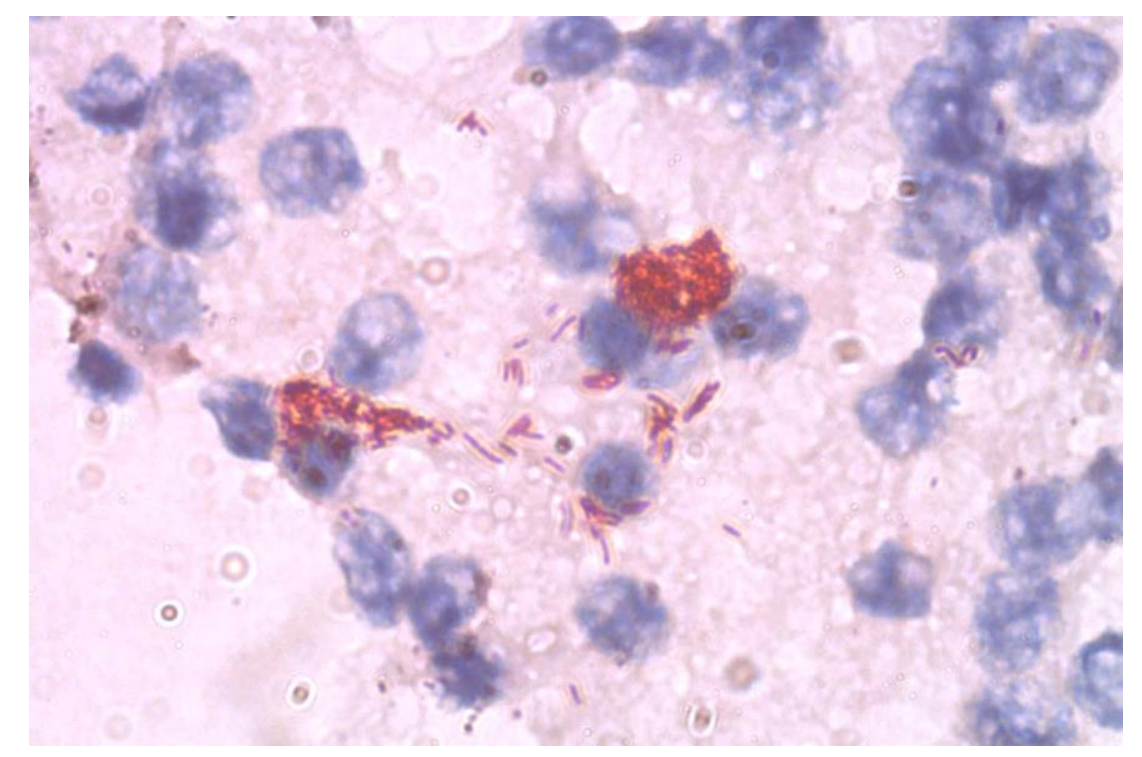

Figure 9: Visualization of MAP in lymphatic tissue using Ziehl-Neelsen staining (Division of Microbiology and Animal Hygiene, Georg-August-University Göttingen)

\section{B) Cultural detection of MAP}

Cultural evidence from faeces and organ samples is still regarded as the "gold standard" for diagnosis (Köhler and Burkert, 2003). However, detection is difficult due to the slow growth of MAP (Lambrecht et al., 1988; Whipple et al., 1991). Various media such as the Herrold's Egg Yolk Medium (HEYM; Figure 10) and liquid culture media (Mycobacterial Growth Indicator Tube [MGIT]) are currently available for bacterial culture (Hughes et al., 2001). A further problem encountered in culturing is decontamination (Stabel, 1996). In order to be able to detect the slowly-growing pathogen, accompanying flora such as bacteria and fungi must be inhibited by the addition of for example Hexadodecylpyridinium chloride (HPC) (Collins, 1996). A positive MAP culture is considered to be $100 \%$ specific when a subsequent hedging by 
for example PCR is carried out. The diagnostic sensitivity is strongly dependent on the disease stage of the animal, and is approximately 50\% (Sherman et al., 1984; Sockett et al., 1992; Stabel, 1998). Further factors influencing the sensitivity of the test are the pathogen concentration in the sample, the storage and processing conditions, and the cultivation method used (Eamens et al., 2000).

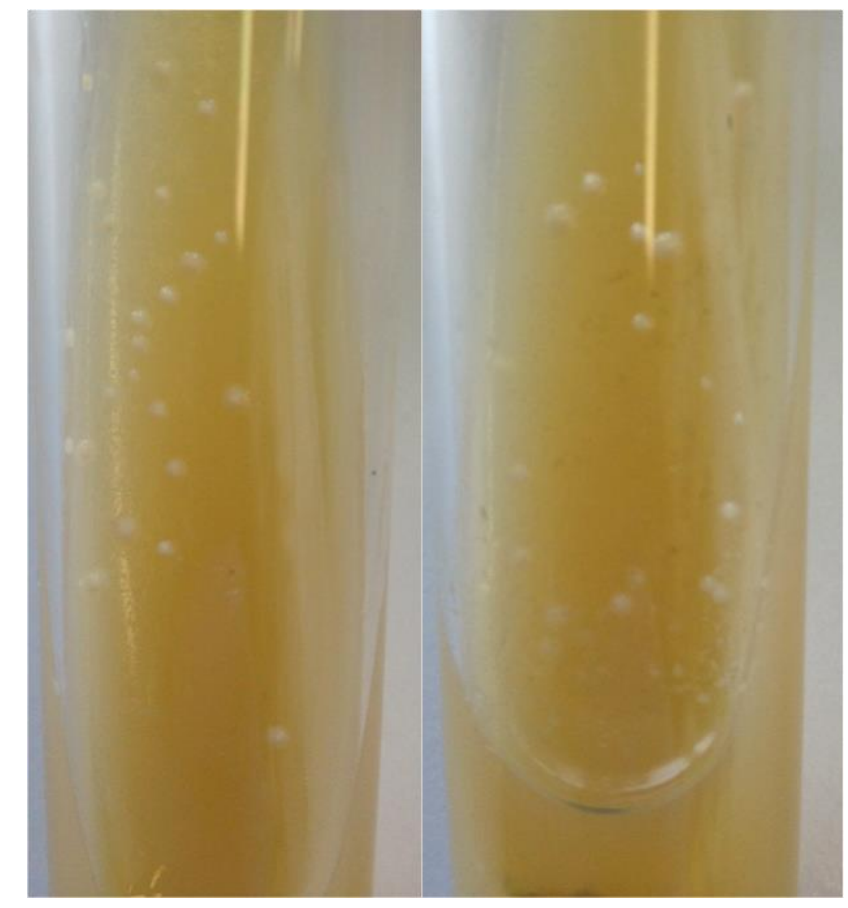

Figure 10: Growth of MAP colonies on HEYM (Division of Microbiology and Animal Hygiene, Georg-August-University Göttingen)

\section{C) Polymerase chain reaction (PCR)}

Another direct detection method is PCR (polymerase chain reaction). PCR is fast and simple to perform, and a high sample throughput is possible (Köhler, 2005). MAP DNA can be detected from faeces (Whipple et al., 1992), blood (Koenig et al., 1993), various organs (Englund et al., 2001) and lymph nodes (Challans et al., 1994) using PCR.

Several different PCR methods have been developed for the detection of MAP. In the nested and semi-nested PCR, two PCR assays are connected in series. The product of the first PCR run is used as the template for the subsequent PCR run. In this way, it is possible to detect very small amounts of DNA. However, the reaction vessels must be 
opened after the first PCR run, increasing the risk of contamination. This risk is significantly lower when using real-time PCR; the workload and time required are also reduced. Real-time PCR is an automated method in which quantification is performed by fluorescence measurement during a PCR cycle.

Most PCRs are based on the MAP-specific insertion sequence IS900, which was discovered by Green et al. (1989). This sequence is $1453 \mathrm{bp}$ in length and is present in 17 largely identical copies within the MAP genome (Li et al., 2005). Analysis of bovine faecal samples revealed diagnostic sensitivities of $54 \%, 49 \%, 48 \%$ and a specificity of $100 \%$ using three different PCR methods, which were based on the IS900 genes (Taddei et al., 2004). Other MAP-specific insertion sequences are ISMav2 (Strommenger et al., 2001), ISMap02 (Stabel and Bannantine, 2005), the hspX gene (Ellingson et al., 1998), ISMpa1 (Olsen et al., 2004), IS_MAP04 (Li et al., 2005) and the F57 sequence (Vansnick et al., 2004).

\section{D) Isothermal amplification}

Methods of DNA amplification at a constant temperature are called isothermal amplification ( $\mathrm{Li}$ and Macdonald, 2015). Many different technologies to accomplish this have been established in the last 20 years, for example loop-mediated isothermal amplification (LAMP) (Notomi et al., 2000), nicking enzyme amplification reaction (Van Ness et al., 2003), rolling circle amplification methods (Gusev et al., 2001), helicase dependent amplification (Vincent et al., 2004) and recombinase polymerase amplification (RPA) (Piepenburg et al., 2006). LAMP is the most widely used method (Cook et al., 2015; Hopkins et al., 2013; Morris et al., 2015; Polley et al., 2013). LAMP is performed at $60{ }^{\circ} \mathrm{C}$, and the results are obtained after 50-60 minutes. However, LAMP utilizes six primers, which are difficult to design, especially for highly variable pathogens. The LAMP method does not include a probe, which leads in some cases to nonspecific results. In contrast, RPA is extremely fast (three to ten minutes), runs at 42 ${ }^{\circ} \mathrm{C}$ and uses two primers and one exo-probe. Amplification in the RPA depends on the binding of the recombinase to the oligonucleotide primers (Figure 11A). The complex then scans the template DNA for the corresponding sequence and initiates $5^{\prime}$-strand invasion of the oligonucleotide at the site of homology. The strand invasion is stabilized by the single-strand binding protein interacting with the complementary DNA strand to avoid primer ejection. Primer extension ensues via a strand-displacing DNA polymerase (Piepenburg et al., 2006). Real-time detection of RPA amplicons is possible via exo- 
probes (Euler et al., 2012a; Euler et al., 2012b) (Figure 11B). The development of fluorescence depends on the separation of fluorophore and quencher via Exonuclease III cleaving at an internal abasic site mimic (tetrahydrofuran, THF) of the hybridized exoprobe. The fluorescence signal is measured in real-time via a simple point-of-care scanner, weighing $1 \mathrm{~kg}$, fixed in the mobile suitcase laboratory (Abd El Wahed et al., 2013a; Abd El Wahed et al., 2013b; Amer et al., 2013; Piepenburg et al., 2006).

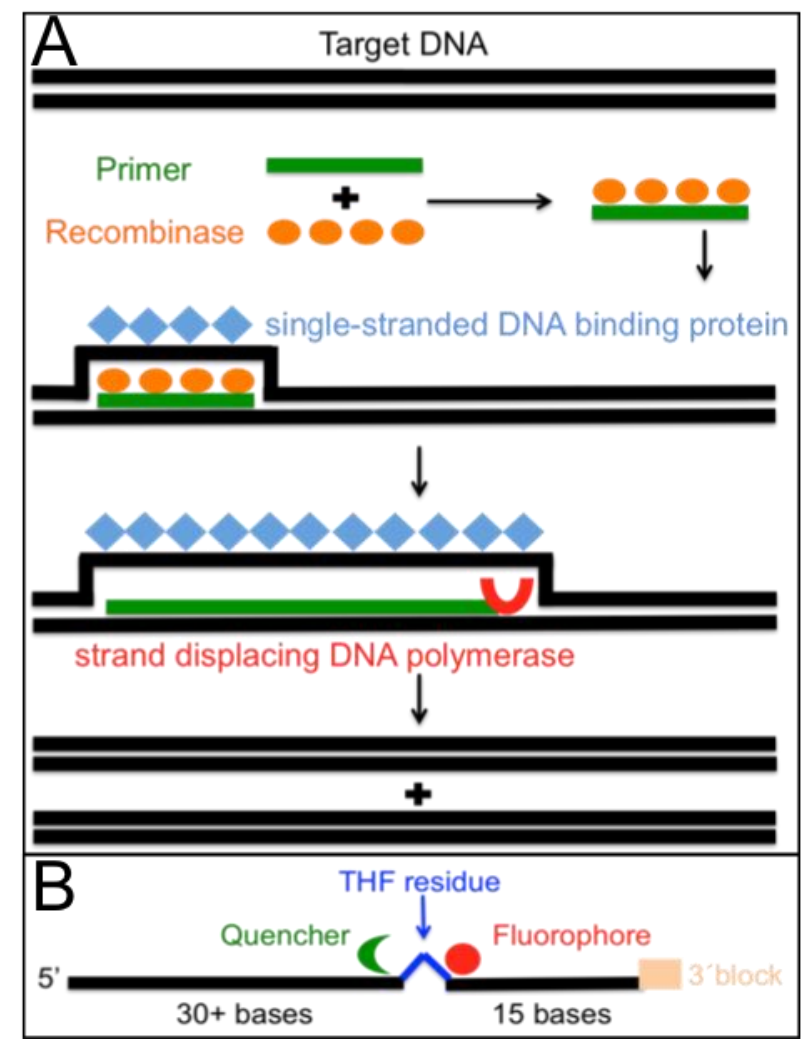

Figure 11: Principle of RPA method. A: The amplification cycle of the RPA showing only the forward primer; B: The structure of the RPA exo-probe for the detection phase of the amplicon (Ahmed Abd El Wahed, Devision of Microbiology and Animal Hygiene, University of Göttingen, Germany)

\section{SCOPE OF THIS THESIS}

The major scope of this thesis is to investigate the unrecognized spread of MAP by asymptomatically infected hosts. These animals do not necessarily exhibit clinical signs and a reliable diagnosis is not available. This leads to a challenge in ensuring efficient 
disease control during the subclinical stage of the disease. Therefore, the occurrence of MAP in a clinically asymptomatic bull as well as different non-ruminant species is examined using different diagnostic methods. Furthermore, possible transmission pathways through the excretion of MAP within animals of the same species and between different species are examined.

Initially, the pattern of MAP occurrence in a naturally infected, but asymptomatic, bull is investigated in chapter I. In this chapter, the presence of MAP is monitored in faeces, blood and semen over a period of four years by qualitative and quantitative PCRs and bacterial culture. Antibody response is additionally measured by commercially available ELISA kits. After necropsy, the distribution of MAP within the subclinical bull is evaluated by PCR and bacterial culture. In chapter II, the susceptibility of non-human primates to MAP 20 animals (belonging to 7 different species) is investigated. After necropsy, faecal and tissue samples are analysed via qualitative and quantitative PCRs and bacterial culture. Only little information are available on the distribution of MAP among wildlife, therefore, chapter III addresses the occurrence of MAP in a group of rock hyraxes. Animals were once captured in South Africa and kept in quarantine in a German zoological garden. Finally, in chapter IV, a recombinase polymerase amplification (RPA) assay is developed for the fast detection of MAP, especially at the point of need. 
STUDIES PERFORMED

2 STUDIES PERFORMED 


\section{CHAPTER I}

\section{Paper published}

Transboundary and Emerging Diseases

\section{Distribution of Mycobacterium avium subsp. paratuberculosis in a Subclinical Naturally Infected German Fleckvieh Bull}

Kim Fechner ${ }^{1}$, Jenny Schäfer ${ }^{1}$, Carola Wiegel ${ }^{1}$, Janina Ludwig ${ }^{1}$, Pia Münster ${ }^{1 \#, ~ A h m a d ~}$ Reza Sharifi ${ }^{2}$, Wilhelm Wemheuer ${ }^{3}$, and Claus-Peter Czerny ${ }^{1 *}$

${ }^{1}$ Division of Microbiology and Animal Hygiene, Institute of Veterinary Medicine, Department of Animal Sciences, Faculty of Agricultural Sciences, Georg-August University, Burckhardtweg 2, D-37077 Göttingen, Germany

${ }^{2}$ Division of Animal Breeding and Genetics, Department of Animal Sciences, Faculty of Agricultural Sciences, Georg-August University, Albrecht-Thaer-Weg 3, D-37075 Göttingen, Germany

${ }^{3}$ Division of Reproduction and Biotechnology, Department of Animal Science, Faculty of Agricultural Sciences, Georg-August University, Burckhardtweg 2, D-37077 Göttingen, Germany

\#Present address. H. Bröring GmbH \& Co. KG, Ladestr. 2, D-49413 Dinklage, Germany.

*Corresponding author. Mailing address: Department of Animal Sciences, GeorgAugust University, Burckhardtweg 2, D-37077 Göttingen, Germany.

Phone: +49 55139 33375. Fax: +49 55139 13513. Email: cczerny@ gwdg.de 


\section{CHAPTER I}

\section{Summary}

Although it has been known for years that Mycobacterium avium subsp. paratuberculosis (MAP) is detectable in the reproductive organs and semen of infected bulls, only few studies have been conducted on this topic worldwide. This study surveyed the MAP status of a bull, naturally infected due to close contact with ist subclinically infected parents over a period of 4 years. From the age of 7 weeks to necropsy, faecal, blood and, after sexual maturity, semen samples were drawn repeatedly. Already at the first sampling day, MAP-DNA was detected in faeces by semi-nested PCR. True infection was confirmed by the detection of MAP-DNA in blood at the age of 40 weeks. In total, MAP-DNA was present in 25\% faecal (34/ 139), $16 \%$ blood (23/140) and 5\% semen (4/89) samples, including MAP-free intervals of up to 9 weeks. MAP genome equivalents (MAP-GE) of up to $6.3 \times 10^{6} / \mathrm{g}$ faeces and $1.8 \mathrm{x}$ $10^{5} / \mathrm{ml}$ blood were determined. Cultivation of MAP occurred only in three of 137 faecal and two of 109 blood, but never in semen samples. Over the whole period, the bull was a serological negative MAP shedder. During necropsy, 42 tissue samples were collected. Neither macroscopic nor histological lesions characteristic of a MAP infection were observed. Cultivation of MAP in tissue sections failed. However, MAPDNA was spread widely in the host, including in tissues of the lymphatic system (7/15), digestive tract (5/14) and the urogenital tract (5/9) with concentrations of up to $3.9 \times 10^{6}$ MAP-GE/g tissue. The study highlighted the detection of MAP in male reproductive organs and semen. It supports the hypothesis that bulls may probably transmit MAP, at least under natural mating conditions. In artificial insemination, this might not be relevant, due to antibiotics included currently in semen extenders. However, the survivability of MAP in this microenvironment should be investigated in detail.

Keywords: Mycobacterium avium subsp. paratuberculosis; pathogenesis; distribution pattern; PCR; German Fleckvieh bull; subclinical infection 


\section{CHAPTER I}

\section{Introduction}

Mycobacterium avium subsp. paratuberculosis (MAP) is the causative agent of Johne's disease, which mainly infects domestic ruminants (cattle, sheep and goats). It is characterized by a degenerative chronic granulomatous inflammation of the digestive tract. The resulting profuse diarrhoea, emaciation and progressive weight loss have a considerable economic impact on the dairy and beef industry (Raizman et al., 2009).

In general, an important part in the transmission of MAP is the infection of the calf. Newborn calves are mostly affected by the faecal-oral route; colostrum and milk from subclinically (Streeter et al., 1995) or clinically infected cows (Taylor et al., 1981) are further sources for infection. Another possible pathway of the infection represents the airborne transmission of MAP by contaminated bioaerosols (Eisenberg et al., 2011). The role of bulls in the transmission of MAP via semen is still unclear even though it has been known since 1970 that MAP is detectable in the semen and reproductive organs (Larsen and Kopecky, 1970). To our knowledge, the venereal transmission of MAP by semen was never demonstrated in the past. As opposed to this, it is generally accepted that MAP is transmitted vertically from the cow to their foetus in utero (Pribylova et al., 2013). A meta-analysis indicated that the total prevalence of calves infected in utero was 13\% among infected cattle (Whittington and Windsor, 2009). In cows, the pathogen was found in uterus tissue and uterine flush, as well as in foetuses (Lambeth et al., 2004; Hasonova et al., 2009). In bulls with (Larsen and Kopecky, 1970; Larsen et al., 1981; Glawischnig et al., 2004; Khol et al., 2010) and without clinical signs (Ayele et al., 2004; Münster et al., 2013), MAP was detected in the testicle, epididymis, seminal gland, prostate gland and bulbourethral gland as well as their semen. In one of the investigated, asymptomatic bulls (Münster et al., 2013), MAP was intermittently detected $(44.6 \% ; 29 / 65)$ in concentrations of up to 5.7 x $10^{5}$ MAP genome equivalents $/ \mathrm{ml}$ semen over a period of 54 months. Although it has been demonstrated previously that bulls excrete the pathogen via semen, the relevance of breeding bulls in the distribution of MAP, especially when they are in the subclinical stage of the disease, is still unknown. In the past, it has been shown that MAP survived the freezing process and antibiotic treatment customarily used for commercial semen samples at that time (Larsen and Kopecky, 1970).

The aim of this study was to characterize the distribution of MAP in faecal, blood and semen samples of an asymptomatic German Fleckvieh bull, which was naturally 
infected by its subclinically infected parents, over the period of 4 years from the age of 7 weeks to necropsy. The analysis of tissue samples, taken during necropsy, provides a detailed overview of the MAP distribution within a subclinical male bovine host.

\section{Materials and Methods}

\section{Animal}

The German Fleckvieh bull (related to Simmental; Bos primigenius taurus) was born on 29 January 2009 in the 4th calendar week (CW) at the Institute of Veterinary Medicine (University of Göttingen, Germany). The infection progress of the bull's subclinically infected father was published already (Münster et al., 2013). The subclinically infected mother tested positive for MAP in blood and faeces during routine controls, but an antibody response was not measured (data not shown). During the suckling period of the bull calf, the parents were tethered and the calf could move freely in a conventional stable. After the suckling period, the bull calf was isolated in quarantine. Feeding and environmental conditions were maintained. By routine diagnostic testing, infections with BHV1, BVD, BT, brucellosis, leucosis, leptospirosis and Q-fever were excluded.

\section{Longitudinal study}

From the age of 7 weeks to necropsy (CW 11 in 2009 to CW 15 in 2013), samples were taken on 142 different dates (Fig. 1). In the first 2 years (CW 11 in 2009 to CW eight in 2011), samples were taken at irregular intervals from 1 to 17 weeks. Until CW 21 in 2010, blood and faeces were collected in parallel, with the exception of 3 days (CW 20, CW 36 and CW 45 in 2009). Once the bull was sexually mature (CW 22 in 2010), semen samples were also drawn repeatedly. From CW 9 in 2011, the study was extended and sampling from all three matrices occurred on a weekly basis. From CW 41 in 2012 until the end of the study, no more semen samples could be collected because the bull was showing aggressive behaviour towards the animal caregivers. In total, there were blood samples taken on 140 days, faecal samples on 139 days and semen samples on 89 days. To avoid faecal cross-contaminations, the order of sample collection was consistently the same; first semen, followed by blood withdrawal, and finally rectal faecal sampling. Prior to sampling, the bull and the teaser were sheared routinely and washed regularly including the genital area. For semen collection, the bull was mounted 
on the teaser using a washed artificial vagina disinfected with $70 \%$ ethanol. Semen samples were collected in sterile tubes. Afterwards, blood was collected from the coccygeal vein. Skin was cleaned and disinfected with $70 \%$ ethanol before. Finally, faecal samples were taken directly from the rectum using sterile disposable gloves and transferred to sterile plastic bottles $(250 \mathrm{ml})$. After collection, all samples were transported to the laboratory. Immediately, all collecting receptacles were cleaned externally and disinfected with $70 \%$ ethanol. Sample preparation was performed in the same order as mentioned for collection.

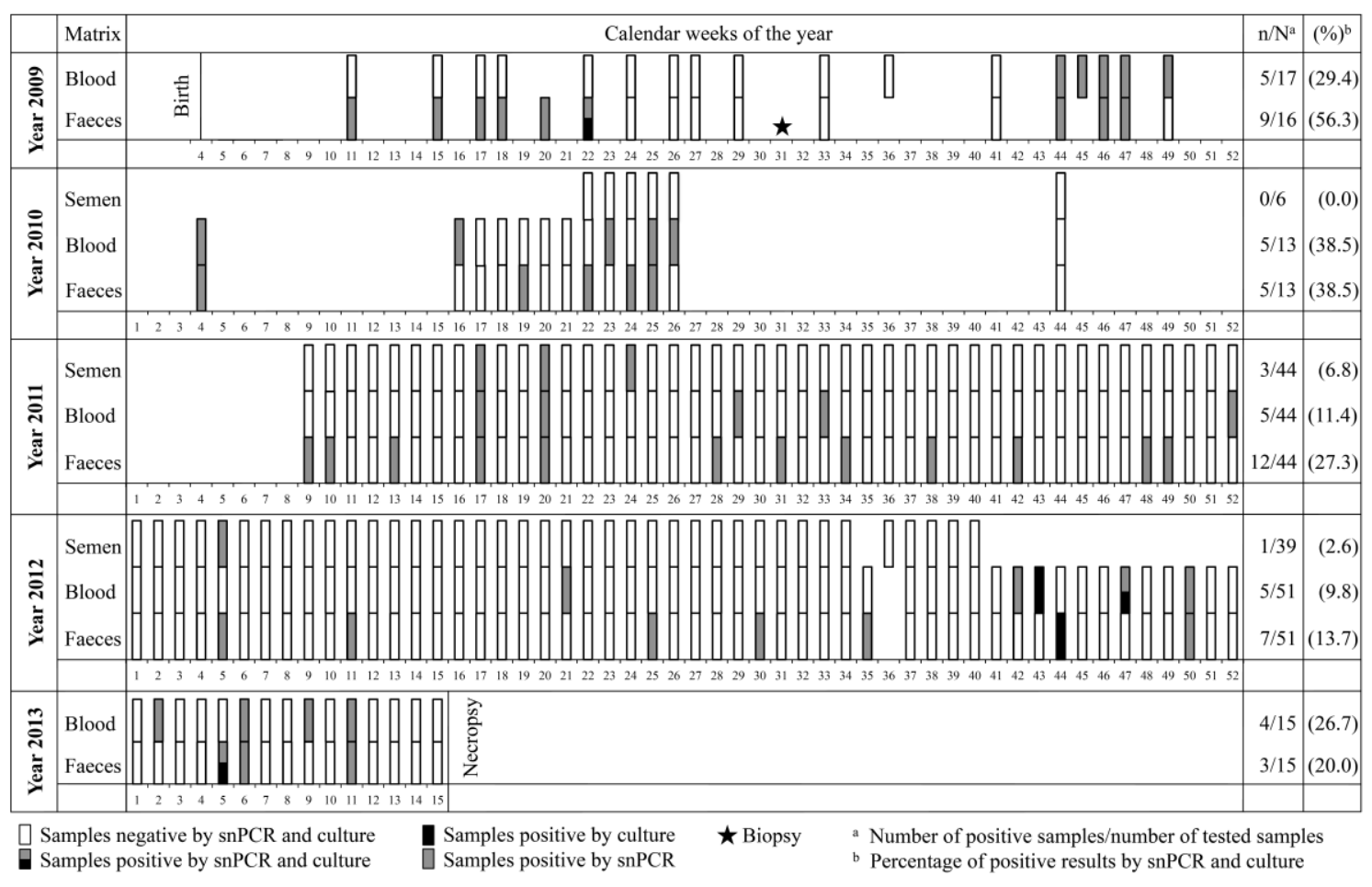

Fig. 1. Distribution patterns of Mycobacterium avium subsp. paratuberculosis in faeces, semen and blood of a naturally infected German Fleckvieh bull over the period of 4 years from birth to necropsy. The bars illustrate each sampling result and date for faecal (bottom), blood (central) and semen samples (top).

\section{Biopsy}

At the age of 27 weeks (CW 31 in 2009), biopsy samples of the ileocaecal lymph nodes, caecum lymph nodes and distal jejunal lymph nodes were drawn at the Veterinary School of Hannover by routine endoscopic surgery. 


\section{CHAPTER I}

\section{Dissection}

At the age of 4 years and 2 months (CW 15 in 2013), the bull was sedated with $10 \mathrm{ml}$ Xylacin $^{\mathrm{TM}}(20 \mathrm{mg} / \mathrm{ml})$ and intravenously euthanized with $30 \mathrm{ml} \mathrm{T61}{ }^{\circledR}$ (Intervet $\mathrm{GmbH}$, Unterschleissheim, Germany). Forty-two tissue samples were collected during necropsy of the bull by professional veterinary staff (Table 3). They included tissue from the lymphatic system (No. 1-15), digestive tract and appending organs (No. 16-29), urogenital tract (No. 30- 38), nervous system (No. 39 and 40) and thorax organs (No. 41 and 42). To avoid cross-contaminations, new, sterile surgical gloves, plastic forceps and scalpels were used for each tissue sample.

\section{Histopathology and immunohistochemistry}

Aliquots of the above-mentioned tissues were fixed with $4 \%$ neutral buffered formaldehyde and embedded in paraffin. Paraffin sections stained with haematoxylin and eosin were examined for histological lesions. Mycobacteria were labelled in paraffin sections by indirect immunoperoxidase staining using a polyclonal anti-MAP immune serum (Dako, Glostrup, Denmark) as described (Krüger et al., 2015). The primary antibody was replaced by an unrelated polyclonal antiserum in consecutive sections as negative control. Tissues from a cow with lesions of paratuberculosis were included as positive control.

\section{Culture}

Mycobacterium avium subsp. paratuberculosis growth in culture by standard procedures is a usual application in our routine diagnostic unit. The methods are accredited according to the standard DIN EN ISO/IEC 17025:2005. The preparation of faecal and tissue samples was performed as described in the official manual of diagnostic procedures published by the Friedrich-Loeffler-Institut (FLI), the German Federal Research Institute of Animal Health (Friedrich-Loeffler-Institut, 2014). However, the homogenization method used for necropsy tissue samples was modified, using the Precellys_ 24 homogenizer. The preparation of the blood and semen samples was adapted on base of the official FLI protocol. The validity of the used method was confirmed for faecal samples by several inter-laboratory proficiency tests organized by the German FLI reference laboratory for mycobacteria. From the age of 7 weeks to necropsy, all collected faecal samples $(n=137)$ were prepared for cultivation, with the exception of 2 days (CW 33 and 49 in 2009). From CW 24 in 2010 to CW 41 in 2012, 
semen samples $(n=87)$ were prepared for cultivation. Blood samples $(n=109)$ were included since CW 10 in 2011, too.

Faecal, semen, blood, biopsy and necropsy tissue samples were immediately prepared for bacterial cultivation on Herrold's egg yolkmedium with mycobactin (HEYM, BD $\mathrm{BBL}^{\mathrm{TM}}$, Heidelberg, Germany). The homogenizations of the biopsy tissue samples (1 g), taken from the 27-week-old calf, were carried out by trituration with sea sand. As opposed to this homogenization, the necropsy tissue samples $(2.5 \mathrm{~g})$ were transferred in 7-ml Precellys_tubes (Precellys_Ceramic Kit 2.8 mm, Peqlab, Erlangen, Germany), before $2.5 \mathrm{ml}$ PBS was added and the samples were homogenized twice in a Precellys ${ }^{\circledR} 24$ homogenizer (Peqlab, Erlangen, Germany) for $30 \mathrm{~s}$ and $6000 \mathrm{rpm}$. Faecal ( $3 \mathrm{~g}$ ), semen and blood (3-5 ml) samples were homogenized by thorough vortexing. For decontamination purposes, the prepared faecal aliquots and biopsy tissue homogenates were suspended in $30 \mathrm{ml}$ of $0.75 \%$ hexadecylpyridinium chloride (HPC) solution, whereas the prepared semen and blood fluids as well as necropsy tissue homogenates were diluted with HPC solution using a ratio of 1:5. All preparations were mixed for 30 min and incubated for $24 \mathrm{~h}$ at room temperature. Afterwards, the supernatants were decanted down to $1 \mathrm{ml}$, before $0.2 \mathrm{ml}$ of the sediments was inoculated to HEYM under sterile conditions and incubated at $37^{\circ} \mathrm{C}$. From the faecal sediment three HEYM tubes and fromblood, semen and tissue sediment two HEYM tubes were inoculated in parallel. In addition, faeces mixed with a MAP reference strain (ATCC 19698, Leibnitz Institute DSMZ, German Collection of Microorganisms and Cell Cultures, Braunschweig, Germany) as well as MAP-positive faeces from a clinically diseased cow were analysed as positive control samples. Bacterial growth was monitored for the first time after 4 weeks and afterwards regularly every 4 weeks for up to 16 weeks. Cultures exhibiting suspicious colonies were subcultured on HEYM - with and without mycobactin - to confirm slow growth rate and mycobactin dependence. Furthermore, growths of bacterial cultures were verified by a MAP-specific snPCR (Münster et al., 2013).

\section{DNA extraction}

DNA extraction and purification of all collected faecal, blood and semen samples for PCR analysis were performed using a modified protocol of the QIAamp Blood Kit (Qiagen, Hilden, Germany), as described by Münster et al. (2013). Faecal samples were 
mixed 1:1 with PBS and filled into four aliquots of $1 \mathrm{~g}$, respectively. Semen and blood samples without PBS were separated into two aliquots of $1 \mathrm{ml}$.

From tissue samples taken during biopsy and necropsy, DNA was extracted using a modified protocol of the QIAamp DNA Mini Kit (Qiagen). Purification of DNA from biopsy tissue samples was performed as described by Münster et al. (2011). The entire range of biopsy tissue materials was analysed (3 x $1 \mathrm{~g}$ of ileocaecal lymph nodes, $2 \times 1$ $\mathrm{g}$ of caecum lymph nodes, $6 \mathrm{x} 1 \mathrm{~g}$ of distal jejunal lymph nodes). As low concentrations of MAP and the formation of clusters were expected, necropsy tissue samples were divided into 12 aliquots of $0.05 \mathrm{~g}$, respectively. Only two aliquots were purified from the retropharyngeal lymph nodes and only ten aliquots from the pancreaticoduodenal lymph nodes, because little tissue material was available. All tissues were transferred to 2-ml Precellys_tubes (Precellys ${ }^{\circledR}$ Ceramic Kit 1.4, 2.8 mm, Peqlab, Erlangen, Germany) containing $160 \mu \mathrm{l}$ PBS. Soft tissue aliquots were homogenized for $30 \mathrm{~s}$ at $6500 \mathrm{rpm}$. Hard or elastic tissues were homogenized three times for $30 \mathrm{~s}$ at $6500 \mathrm{rpm}$ in the

Precellys ${ }^{\circledR} 24$ device, after which $200 \mu$ tissue lysis buffer (ATL buffer) and $40 \mu 1$ ready-to-use proteinase $\mathrm{K}$ solution $(20 \mathrm{mg} / \mathrm{ml})$ were added. Mixed samples were incubated on a thermomixer at $56^{\circ} \mathrm{C}$ and $900 \mathrm{rpm}$ for $1 \mathrm{~h}$ followed by adding $400 \mu \mathrm{l}$ lysis buffer (AL buffer). The tubes were incubated on the thermomixer again, but at $70^{\circ} \mathrm{C}$ and $900 \mathrm{rpm}$ for $10 \mathrm{~min}$. After centrifugation (20 $\left.000 \mathrm{~g}, 1 \mathrm{~min}\right)$, the supernatants were transferred into new tubes before $400 \mu \mathrm{l}$ of ethanol $(\geq 99.8 \%)$ was added. Loading of the QIAamp Spin Column and washing procedures were performed according to the manufacturer's instructions. Finally, the DNA was eluted in $200 \mu \mathrm{l}$ elution buffer at $85^{\circ} \mathrm{C}$ and stored at $-20^{\circ} \mathrm{C}$ prior to use.

\section{Qualitative semi-nested polymerase chain reaction}

A qualitative semi-nested PCR (snPCR), based on the insertion sequence IS900, was performed as described previously (Münster et al., 2011). The primers MAPfor1 (5'GTC GGC GTG GTC GTC TGC TGG GTT GAT-'3) and MAPrev (5'-GCG CGG CAC GGC TCT TGT TGT AGT C-'3) amplified a 587-bp fragment in the first run. The forward primer MAP for 2 (5'- CGG GCG CAC GGT CCA TCA CG'3), together with the primer MAPrev, was taken for amplification of a 278-bp fragment in the second step. MAP-DNA extracted from the MAP reference strain ATCC 19698 was included as a positive control in each PCR run, whereas sterile distilled water was used as a 
negative control. The snPCR used is well validated, and the primers were designed to bind to highly MAP-specific sites of the IS900 (Münster et al., 2011, 2013). The snPCR has a detection limit of $2.5 \mathrm{fg}$ MAP-DNA (one genome equivalent/reaction). After PCR, $5 \mu \mathrm{l}$ of the amplicons was mixed with $2 \mu 1$ sample buffer and separated on $1 \%$ agarose gels for $1 \mathrm{~h}$ at $110 \mathrm{~V}$. Subsequently, amplicons were stained with ethidium bromide $(0.5$ $\mu \mathrm{g} / \mathrm{ml}$ ) before PCR products were visualized under UV light. To preclude crosscontamination, DNA isolation, preparation of master mixes, addition of template DNA and electrophoresis were carried out in separate laboratories.

\section{Quantitative real-time polymerase chain reaction}

For quantification of MAP-DNA, a new quantitative realtime PCR (qPCR) assay based

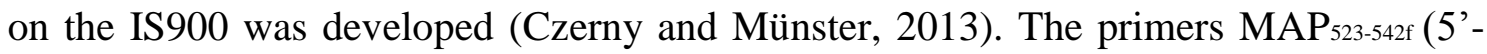
TAC CGC GGC GAA GGC AAG AC-3') and MAP $661-642 r$ (5' -CGG AAC GTC GGC TGG TCA GG-3') were applied (MWG Biotech, Ebersberg, Germany) to amplify a 139-bp fragment. The TaqMan probe MAP 617-636p (5'-FAM-ATG ACA TCG CAG TCG AGC TG-BHQ1-3') was designed to anneal on the PCR target allowing detection of specific PCR products. Amplification and fluorescence detection were performed on the Light Cycler $^{\circledR} 480$ System using 96-well PCR plates (Roche Molecular Diagnostic, Mannheim, Germany). A total reaction volume of $20 \mu \mathrm{l}$ per well was used containing 10 $\mu 1$ Light Cycler ${ }^{\mathrm{TM}} 480$ Probes Master (Roche Diagnostics, Mannheim, Germany), $0.5 \mu 1$ of each upstream and downstream primer (10 pmol/ $\mu 1), 1 \mu 1$ MAP $617-636 \mathrm{P}-$ probe $(10$ $\mathrm{pmol} / \mu \mathrm{l})$, as well as $3 \mu \mathrm{l}$ sterile water and $5 \mu \mathrm{l}$ of the extracted sample DNA. The amplification process started with an initial pre-incubation step at $95^{\circ} \mathrm{C}$ for $10 \mathrm{~min}$, followed by 40 cycles at $95^{\circ} \mathrm{C}$ for $15 \mathrm{~s}, 60^{\circ} \mathrm{C}$ for $30 \mathrm{~s}$ and $72^{\circ} \mathrm{C}$ for $35 \mathrm{~s}$. Fluorescence was measured within the FAM channel after the annealing step of each cycle. The qPCR was performed with all aliquots that tested MAP positive by snPCR. Samples were tested in three independent qPCR runs. Validation data have shown that the qPCR, under the used conditions, had a detection limit of $50 \mathrm{fg} /$ reaction (10 genome equivalents/reaction) and a quantification limit of $5 \mathrm{pg} /$ reaction MAP-DNA (945 genome equivalents/reaction). As a positive control and for the quantification of MAPDNA concentration, an MAPDNA standard curve (MAP reference strain ATCC 19698) was titrated in $\log _{10}$ steps $(1 \mathrm{ng} / \mu \mathrm{l}$ to $1 \mathrm{fg} / \mu \mathrm{l})$, whereas sterile distilled water was used as a negative control. MAP genome equivalents (MAP-GE) detected by qPCR were calculated from the MAP K-10 genome size of 4.829.781 bp (Li et al., 2005). Assuming 
the molecular weight of one base pair is $660 \mathrm{~g} \mathrm{x} \mathrm{mol}^{-1}$ and the Avogadro number is $6.022 \times 10^{23} \mathrm{~mol}^{-1}$, calculations resulted in one MAP-GE of $5.29 \mathrm{fg}$ of MAP-DNA. The formula for calculating the number of MAP-GE in blood or semen per $\mathrm{ml}$ and in faecal or tissue sample per $\mathrm{g}$ was as follows: Concentration of DNA $(\mathrm{fg} / \mu \mathrm{l}) \mathrm{x}$ volume of eluted DNA (200 $\mu \mathrm{l}) / 5.29 \mathrm{fg}$ x 1 (blood and semen sample); x 2 (faecal sample); x 20 (tissue sample $)=$ MAP-GE per extracted sample aliquot, per $\mathrm{ml}$ or g sample, respectively.

\section{Enzyme-linked immunosorbent assay}

In total, 139 blood samples were analysed by a commercial indirect ELISA following the instructions of the manufacturer (IDEXX Paratuberculosis Screening, IDEXX Laboratories, Germany/ELISA Paratuberculosis Screening, Institute Pourquier, France). On 1 day of the investigation period, no ELISA could be performed (CW 44 in 2010). The evaluation of the serum samples was performed according to the cut-off recommended by the manufacturer: serum samples $\leq 60 \% \mathrm{~S} / \mathrm{P}$ negative $(-),>60 \%-$ $<70 \% \mathrm{~S} / \mathrm{P}$ borderline $( \pm), \geq 70 \% \mathrm{~S} / \mathrm{P}$ positive $(+)$. Since 10 March 2010, the cut-off of the ELISA has been modified by the manufacturer: serum samples $\leq 45 \% \mathrm{~S} / \mathrm{P}$ negative $(-),>45 \%-<55 \%$ S/P borderline $( \pm), \geq 55 \%$ S/P positive $(+)$. Plates were read using an automated ELISA plate reader (Sunrise ${ }^{\mathrm{TM}}$, Tecan Trading AG, Switzerland). Samples were analysed in duplicates, and the calculated S/P means were recorded.

\section{Statistical analysis}

Statistical analysis of the longitudinal data set was carried out by application of a linear logistic regression with a binary response variable, which is modelled as a binomial random variable $\left(y_{i}\right)$. The data were then analysed with the GLIMMIX procedure of SAS (Littell et al., 1999) using the following model:

$$
\log \text { it }\left(\frac{\pi_{\mathrm{r}}}{1-\pi_{\mathrm{r}}}\right)=\eta_{\mathrm{r}}=\beta_{0}+\beta_{1} \mathrm{x}_{\mathrm{i}}
$$

where $\pi_{\mathrm{i}}$ is the probability of detecting MAP-DNA in the respective matrix (faeces, blood or semen) from birth to necropsy by snPCR, $\beta_{0}$ and $\beta_{1}$ represent the logistic regression parameters, and $\mathrm{x}_{\mathrm{i}}$ signifies the explanatory time variable. The regression lines are fitted by calculating back-transformed logistic regression parameters. The estimates based on the inverse link function $\pi=\exp (x \beta) /[1+\exp (x \beta)]$ to the original scale (probability). 
In addition, the chi-square statistics $\left(\chi^{2}\right)$ and the corresponding Phi-coefficients $(\phi)$ were calculated to analyse the associations between the snPCR results of the MAP-DNA occurrence in faeces, blood and semen by snPCR using the FREQ procedure of SAS 9.4 software (SAS Inst., Inc., Cary, NC, USA, 2014). Calculation of the chi-square values was only performed if the frequencies were $>5$. Values of $\mathrm{P} \leq 0.05$ were considered significant for all tests. The Phi-coefficient is used to measure the strength of association between two binary variables. The level of association of the Phi-coefficient values was defined as follows: $\phi=0.00$ no association, $0.00>\phi \leq 0.25$ weak association, $0.25>\phi \leq 0.66$ moderate association, $0.66>\phi<1.00$ strong association and $1.00=/$ perfect association .

\section{Results}

\section{Longitudinal study}

The general condition of the investigated German Fleckvieh bull was good, and the faecal consistency was normal throughout the whole investigation period. The bull did not show any signs of paratuberculosis. However, it must be stated that the bull remained small in size for its age and showed poor growth performance similar to its father. On the day of the necropsy, with an age of 4 years and 2 months, the bull weighed $642 \mathrm{~kg}$ at a height of $140 \mathrm{~cm}$.

\section{Detection of MAP-DNA in faecal, blood, semen and biopsy tissue samples by snPCR}

At the first sampling day, at an age of 7 weeks (CW 11 in 2009), MAP-DNA was detected in faeces (Fig. 1). At the age of 27 weeks (CW 31 in 2009), biopsy samples were taken. No MAP-DNA was detected in any of the samples, including those from the ileocaecal lymph nodes, caecum lymph nodes and distal jejunal lymph nodes. At the age of 40 weeks (CW 44 in 2009), MAP-DNA was detected in blood for the first time. At the age of 2 years and 3 months (CW 17 in 2011), the pathogen was ascertained in semen for the first time. Over the course of the study, the results showed an intermittent MAP-DNA detection. Results are shown in detail in Fig. 1. In total, 23 of 140 (16.4\%) blood samples and four of 89 (4.5\%) semen samples tested positive for MAP-DNA by snPCR. The highest number of MAP-DNA-positive samples was detected in faeces $(34 / 139 ; 24.5 \%)$ (Table 1). MAP-DNA was simultaneously detected in faeces and blood 


\section{CHAPTER I}

on 10 of $138(7.3 \%)$ sampling days; in blood and semen on two of $88(2.3 \%)$ days; and in faeces and semen on three of 88 days (3.4\%). In semen, blood and faeces, MAPDNA was detected simultaneously on two of 88 (2.3\%) days (Fig. 2). MAP-DNA was not observed in any of the three matrices on 94 of 142 days (66.2\%) and during intervals of up to 9 weeks (CW 12 to 20 in 2012) by snPCR.

Table 1: Frequencies of MAP-DNA detection by snPCR and the corresponding statistical analysis of the Chi-square test $\left(\chi^{2}\right)$ and Phi-coefficient $(\phi)$.

\begin{tabular}{|c|c|c|c|c|c|c|c|}
\hline & & Faeces & Negative & Positive & Total (\%) & $\begin{array}{r}\chi^{2} \\
\text { p value }\end{array}$ & $\phi$ \\
\hline \multirow{3}{*}{$\frac{\mathscr{\theta}}{\mathscr{0}}$} & Frequency (\%) & Negative & $93(67.39)$ & $23(16.67)$ & $116(84.06)$ & & \\
\hline & Frequency (\%) & Positive & $12(8.70)$ & $10 \quad(7.25)$ & $22(15.95)$ & 6.67 & \\
\hline & Total $(\%)$ & & $\begin{array}{r}105 \\
(76.01)\end{array}$ & $33(23.91)$ & $138(100.00)$ & $\mathrm{P}<0.01$ & 0.22 \\
\hline \multirow{4}{*}{$\frac{\bar{g}}{80}$} & & Semen & & & & & \\
\hline & Frequency (\%) & Negative & $77(87.50)$ & $2(2.27)$ & $\begin{array}{ll}79 & (89.77)\end{array}$ & & \\
\hline & Frequency (\%) & Positive & $7 \quad(7.95)$ & $2(2.27)$ & $9 \quad(10.23)$ & n.a. & \\
\hline & Total $(\%)$ & & $84(95.45)$ & $4(4.55)$ & $88(100.00)$ & & \\
\hline \multirow{4}{*}{ 芯 } & & Faeces & & & & & \\
\hline & Frequency (\%) & Negative & $68(77.27)$ & $16(18.18)$ & $84 \quad(95.45)$ & & \\
\hline & Frequency (\%) & Positive & 1 (1.14) & 3 (3.41) & $4 \quad(4.55)$ & n.a. & \\
\hline & Total $(\%)$ & & $69(78.41)$ & $19(21.59)$ & $88(100.00)$ & & \\
\hline
\end{tabular}

n.a. not analyzed

The matrices are indicated in bold. 


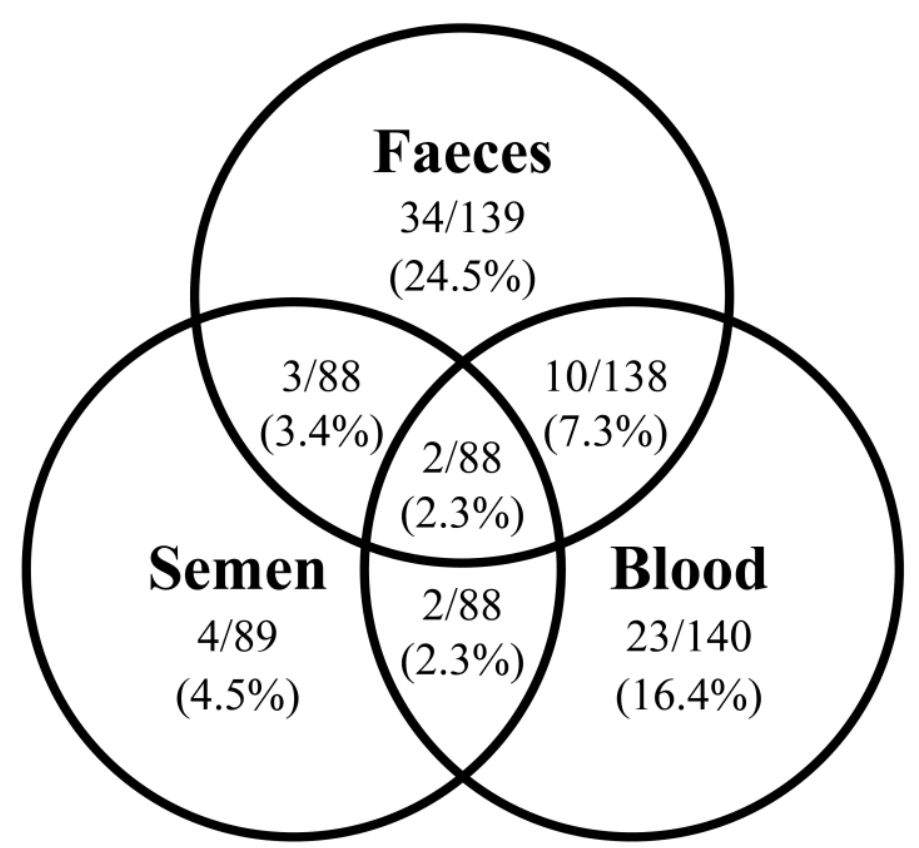

Fig. 2. Venn diagram showing the interrelationships between the detectability of MAPDNA in faeces, semen and blood of a naturally infected German Fleckvieh bull by snPCR. Given are the numbers of positive samples out of the total numbers of samples tested. The percentages of positive tested samples are given in parentheses.

Detection and quantification of MAP-DNA in faecal, blood and semen samples by $q P C R$

The calculated average values of MAP-GE/g or $\mathrm{ml}$, which are based on all positive aliquots and three independent qPCR runs of each sample, are listed in Table 2 in detail. In total, in five of 34 snPCR-positive faecal samples, MAP-DNA was detected by qPCR. In four of them, MAPDNA could be quantified. The amount of MAP-DNA ranged from $2.61 \times 10^{5}$ to $6.25 \times 10^{6} \mathrm{MAP}-\mathrm{GE} / \mathrm{g}$ faeces. Detection but no quantification of MAP-GE was possible in one of the five qPCR-positive faecal samples due to the quantification limit of 5 pg MAP-DNA. In total, in 14 of 23 snPCR-positive blood samples, MAP-DNA was detected using qPCR. In seven of them, MAP-DNA was quantified ranging from $4.07 \times 10^{4}$ to $1.84 \times 10^{5} \mathrm{MAP}-\mathrm{GE} / \mathrm{ml}$. Detection but no quantification of MAP-GE was possible in the remaining seven blood samples. Furthermore, two of four snPCR-positive semen samples were positive by qPCR but quantification failed due to the quantification limit. 


\section{CHAPTER I}

\section{Serological investigations}

Over the whole period of the investigation, 139 sera were tested by the Pourquier/IdexxELISA. The S/P ranged from $0.49 \%$ to $42.48 \%$ and, therefore, no antibody response was measured (serum samples $\leq 45 \% \mathrm{~S} / \mathrm{P}$ negative). Therefore, the bull was a serological negative MAP shedder.

\section{Bacteriological investigation of faecal, blood, semen and biopsy tissue samples}

Over the whole investigation period, only three of 137 faecal samples investigated culturally showed moderate growth of MAP colonies on HYEM with mycobactin (Fig. 1). Furthermore, only from two of 109 culturally investigated blood samples MAP bacteria were isolated. No growth of MAP was ever observed in any of the culturally investigated semen samples $(\mathrm{n}=87)$. Furthermore, MAP was not cultivable from any of the biopsy tissues. 


\section{CHAPTER I}

Table 2. Detection of MAP-DNA by qPCR in faeces, blood and semen collected from a subclinically infected German Fleckvieh bull over a period of 4 years from the age of 7 weeks to necropsy. Detection of MAP-DNA is given as average crossing point with standard deviation and the corresponding average values of MAP genome equivalents in calendar weeks for each year and each analysed matrix

\begin{tabular}{|c|c|c|c|c|}
\hline Matrix & Year & Calendar Week & Mean CP \pm SD & MAP-GE/g or $\mathrm{ml}$ \\
\hline \multirow[t]{5}{*}{ Feces } & 2009 & 11 & $34.04 \pm 1.67$ & positive* \\
\hline & & 15 & $28.00 \pm 0.40$ & $1.52 \times 10^{6}$ \\
\hline & & 17 & $26.29 \pm 0.40$ & $6.25 \times 10^{6}$ \\
\hline & & 47 & $30.38 \pm 0.19$ & $2.61 \times 10^{5}$ \\
\hline & 2012 & 50 & $28.66 \pm 1.38$ & $1.88 \times 10^{6}$ \\
\hline \multirow[t]{14}{*}{ Blood } & 2009 & 44 & $31.16 \pm 0.38$ & $7.88 \times 10^{4}$ \\
\hline & & 45 & $30.59 \pm 1.31$ & $1.81 \times 10^{5}$ \\
\hline & & 46 & $32.37 \pm 0.43$ & positive* \\
\hline & & 47 & $31.99 \pm 0.08$ & positive* \\
\hline & & 49 & $32.64 \pm 0.82$ & $1.40 \times 10^{5}$ \\
\hline & 2010 & 25 & $31.31 \pm 0.40$ & $6.49 \times 10^{4}$ \\
\hline & & 26 & $31.89 \pm 0.06$ & $4.07 \times 10^{4}$ \\
\hline & 2011 & 17 & $31.64 \pm 0.47$ & $5.20 \times 10^{4}$ \\
\hline & & 20 & $35.00 \pm 0.00$ & positive* \\
\hline & & 27 & $35.00 \pm 0.00$ & positive* \\
\hline & & 33 & $35.00 \pm 0.00$ & positive* \\
\hline & 2012 & 50 & $30.97 \pm 1.11$ & $1.84 \times 10^{5}$ \\
\hline & 2013 & 6 & $35.00 \pm 0.00$ & positive* \\
\hline & & 11 & $35.00 \pm 0.00$ & positive* \\
\hline \multirow[t]{2}{*}{ Semen } & 2011 & 17 & $32.06 \pm 0.09$ & positive* \\
\hline & & 20 & $32.03 \pm 0.10$ & positive* \\
\hline
\end{tabular}

* Detection but failure of quantification of MAP genome equivalents due to quantification limit of $5 \mathrm{pg}$ MAP/DNA. 


\section{Statistical analysis}

To analyse the probability of detecting MAP-DNA by SnPCR in the respective matrices (faeces, blood or semen) from birth to necropsy, a statistical analysis of the longitudinal data was carried out by applying a linear logistic regression with a binary response variable (Fig. 3). The probability of detecting MAP-DNA in faeces (56.24\% to $10.73 \%$ ) and semen (11.28\% to $1.22 \%$ ) decreased from birth to necropsy. The probability of detecting MAP-DNA in blood decreased from birth (38.53\%) up to the age of 3 years $(13.22 \%)$, and increased until the day of necropsy to $15.40 \%$.

In addition, to analyse the statistical associations between the snPCR results of the MAP-DNA occurrence in faeces, blood and semen, the chi-square value $\left(\chi^{2}\right)$ was calculated in cases of frequencies $>5$ (Table 1). For semen and blood as well as semen and faeces, no chi-square statistics could be performed, because the frequencies in both cases were $<5$. The calculated chi-square values between faeces and blood were greater than the critical value of 3.84 ( $\mathrm{P}<0.05)$, indicating a statistical association. Following this, the corresponding Phi-coefficients $(\phi)$ were calculated to determine the measure of strength of the association between two matrices. A weak association was calculated between the matrices blood and faeces $(\phi 0.22 ; \mathrm{P}>0.05 ; \mathrm{n}=138)$.

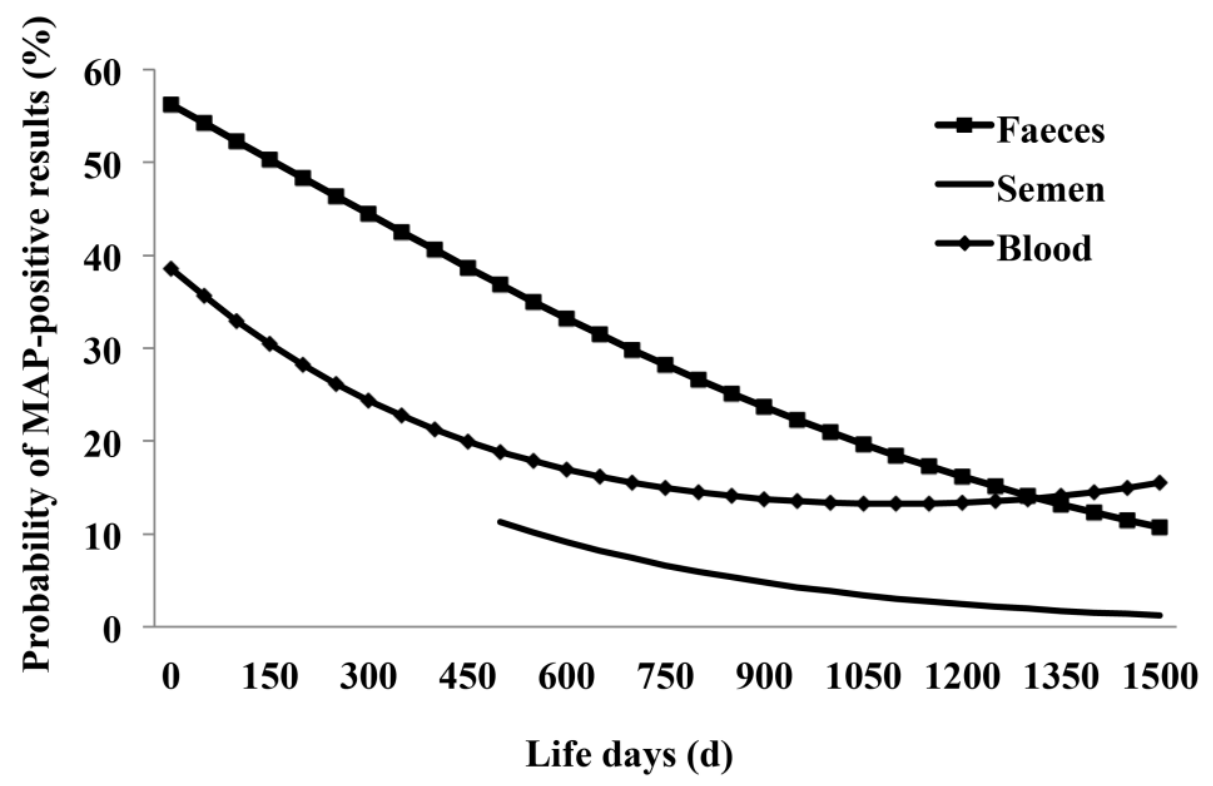

Fig. 3. Statistical analysis of the MAP-DNA detection in the respective matrices (faeces, blood or semen) from birth to necropsy by snPCR was carried out by applying a linear logistic regression with a binary response variable. 


\section{CHAPTER I}

\section{Dissection}

On 08 April 2013 (CW 15), the German Fleckvieh bull was dissected. During necropsy of the bull, no macroscopic abnormalities were observed, which were typical for an infection with MAP.

\section{Detection of MAP-DNA in tissue samples by SnPCR}

In summary, MAP-DNA was detected by snPCR in 17 of 42 tissue samples. In Table 3, the results are listed in detail. In total, seven of 15 (46.7\%) lymphoid organs tested MAP positive, including the right palatine tonsil, retropharyngeal lymph nodes, tracheobronchial lymph nodes, mediastinal lymph nodes, ileocaecal lymph nodes and superficial inguinal lymph nodes. The highest number of MAP-DNA-positive aliquots was detected in the bone marrow (9/12). Furthermore, MAP-DNA was detected in five of $14(35.7 \%)$ tissues of the digestive tract, including tissues from the pancreas, duodenum, caecum, ileum and colon. None of the digestive tract appending organs, organ samples belonging to the head or any samples collected from the jejunum and rectum tested MAP-DNA positive. In total, five of nine (44.4\%) organs of the urogenital tract were confirmed MAP positive by snPCR, including the testicle, epididymis, ampulla of deferent duct, prostate gland and kidney. No MAP-DNA was detected in the seminal gland, bulbourethral gland, adrenal gland or urinary bladder. Furthermore, the nervous system (cerebrum, cerebrospinal fluid) and thorax organs (heart and lung) also tested negative for MAP-DNA.

\section{Detection and quantification of MAP-DNA in tissue samples by qPCR}

Based on all positive aliquots and three independent qPCR runs of each tissue sample, the calculated crossing points - with standard deviations and average values of MAPGE/g tissue - are listed in Table 3 in detail. In total, MAP-DNA was detected in seven of 17 snPCR positive tissue samples by qPCR, whereas MAP-DNA could only be quantified in two of them. The concentration of MAP indicated in the tracheobronchial lymph nodes was $1.55 \times 10^{6} \mathrm{MAP}-\mathrm{GE} / \mathrm{g}$ tissue and in the superficial inguinal lymph nodes $3.86 \times 10^{6} \mathrm{MAP}-\mathrm{GE} / \mathrm{g}$ tissue. MAP-DNA was detected, but due to the quantification limit it could not be quantified in the remaining five tissues, including the ileocaecal lymph nodes, pancreas, duodenum, colon and epididymis. 
Table 3: Detection of MAP-DNA by snPCR and qPCR in tissue samples collected from a subclinically infected German Fleckvieh bull.

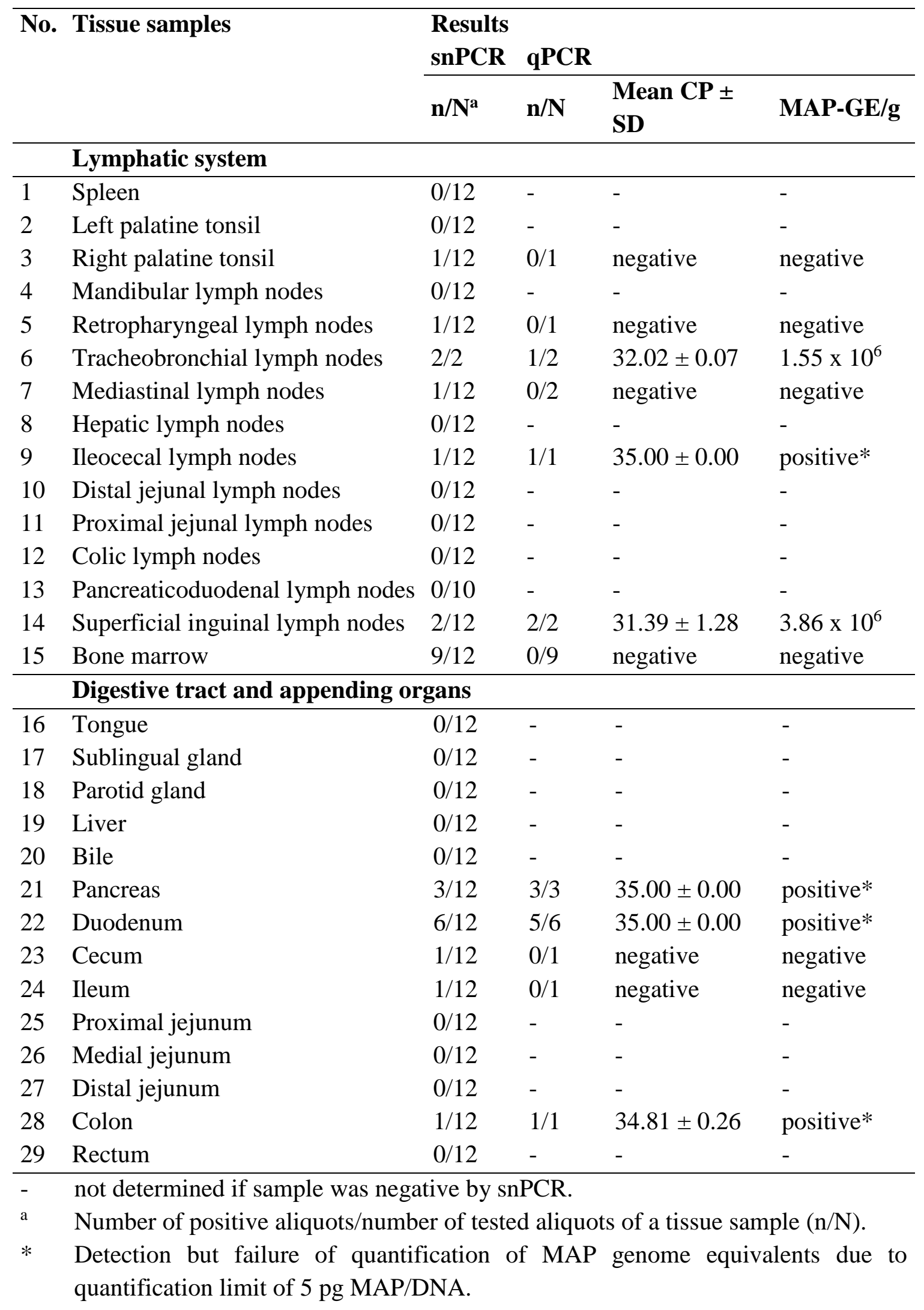


Table 3: continued

\begin{tabular}{|c|c|c|c|c|c|}
\hline \multirow[t]{2}{*}{ No. } & \multirow[t]{2}{*}{ Tissue samples } & \multicolumn{4}{|l|}{ Results } \\
\hline & & $\mathbf{n} / \mathbf{N}^{\mathbf{a}}$ & $\mathbf{n} / \mathbf{N}$ & Mean CP \pm SD & MAP-GE/g \\
\hline & \multicolumn{5}{|l|}{ Urogenital tract } \\
\hline 30 & Testicle & $4 / 12$ & $0 / 4$ & negative & negative \\
\hline 31 & Epididymis & $3 / 12$ & $1 / 3$ & $35.00 \pm 0.00$ & positive* \\
\hline 32 & Ampulla of deferent duct & $1 / 12$ & $0 / 1$ & negative & negative \\
\hline 33 & Seminal gland & $0 / 12$ & - & - & - \\
\hline 35 & Bulbourethral gland & $0 / 12$ & - & - & - \\
\hline 36 & Kidney & $2 / 12$ & $0 / 2$ & negative & negative \\
\hline 37 & Adrenal gland & $0 / 12$ & - & - & - \\
\hline \multirow[t]{2}{*}{38} & Urinary bladder & $0 / 12$ & - & - & - \\
\hline & \multicolumn{5}{|l|}{ Nervous system } \\
\hline 39 & Cerebrum & $0 / 12$ & - & - & - \\
\hline \multirow[t]{2}{*}{40} & Cerebrospinal fluid & $0 / 12$ & - & - & - \\
\hline & \multicolumn{5}{|l|}{ Thorax organs } \\
\hline 41 & Heart & $0 / 12$ & - & - & - \\
\hline 42 & Lung & $0 / 12$ & - & - & - \\
\hline- & \multicolumn{5}{|c|}{ not determined if sample was negative by snPCR. } \\
\hline 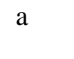 & \multicolumn{5}{|c|}{ Number of positive aliquots/number of tested aliquots of a tissue sample $(\mathrm{n} / \mathrm{N})$. } \\
\hline$*$ & \multicolumn{5}{|c|}{$\begin{array}{l}\text { Detection but failure of quantification of MAP genome equivalents due to } \\
\text { quantification limit of } 5 \mathrm{pg} \text { MAP/DNA. }\end{array}$} \\
\hline
\end{tabular}

\section{Histopathology and immunohistology in tissue samples}

Lesion characteristics of paratuberculosis were not detected in any of the examined tissue samples. Small groups of pigment-laden macrophages were present in the cortex of hepatic, tracheobronchial, pancreaticoduodenal, distal and proximal jejunal, colici and ileocaecal lymph nodes. Small round foci of mineralization and not demarcated by inflammatory cells were found in one jejunal lymph node and the left tonsil. Small multifocal abscesses with extensive central necrosis, feed plant material, bacterial colonies and surrounded by neutrophils were located deep in the crypts of the left tonsil. Specific labelling for mycobacteria was seen in close association to the plant material and the surrounding neutrophils of these abscesses. All other tissues were negative for mycobacterial antigens. 


\section{CHAPTER I}

\section{Bacteriological investigation in tissue samples}

During the necropsy of the bull, 42 tissue samples in total were immediately prepared for bacterial culture, but MAP colonies could not be cultivated on HEYM with mycobactin after 16 weeks.

\section{Discussion}

The infection of cattle with MAP starts mainly during the first months of life as a result of contact between newborn calves and contaminated colostrum, milk or faeces. Although excretion of MAP in the faeces and colostrum of its mother was not monitored during that period and an intrauterine infection cannot be ruled out completely, because no diagnostic data were collected from the calf immediately after birth, it is most likely that the bull investigated here over a period of 4 years was obviously infected naturally with MAP by its subclinically infected parents. The repeated detection of MAP-DNA in faeces samples of its father with concentrations of up to $1.5 \times 10^{5}$ MAP-GE/ g faeces during the suckling period (Münster et al., 2013) supports an infection by the faecal-oral route or by bacterial loaded air. MAP-DNA was detected repeatedly in the examined bull with concentrations of up to $6.25 \times 10^{6} \mathrm{MAP}$ $\mathrm{GE} / \mathrm{g}$ faeces between the ages of 7 and 16 weeks. Sporadic faecal shedding of MAP has been reported previously in experimentally infected calves younger than 8 months (Collins and Zhao, 1994; McDonald et al., 1999). However, it is difficult to differentiate between a true infection (active MAP shedding) and a pass through (passive MAP shedding) of MAP (van Roermund et al., 2007). In the examined bull, it is possible that the pathogen was shed passively during the first weeks of life. This assumption was confirmed by the MAP-negative biopsy samples, which were collected at the age of 27 weeks. True infection of the bull was confirmed by the detection of MAP-DNA in blood at the age of 40 weeks. From this point onwards, MAPDNA was detected repeatedly in high concentrations of up to $1.81 \times 10^{5}$ MAP-DNA equivalents $/ \mathrm{ml}$ over a period of 5 weeks in blood. This indicated that the pathogen has passed the boundary from the intestine to other body regions.

Following the infection with MAP, animals normally enter a silent phase for at least 2 years in which no clinical symptoms are evident. During this stage, the pathogen is intermittently shed in faeces, but most of the time it is below the detection limit of 
conventional methods (Whitlock and Buergelt, 1996). In the present study, MAP-DNA was detected intermittently by PCR in all three matrices (faeces, blood and semen). Within the first 2 years, 13 of 29 faecal (44.83\%) and 10 of 30 blood samples (33.33\%) tested positive for MAP-DNA. Additionally, the regression analysis of MAP-DNA detection had offered a higher probability in faeces and blood within the first 2 years of life. With increasing age, the probability of MAP-DNA detection decreased in all three matrices. However, the analysis is based on the distribution pattern of one animal and individual differences in the reactions to MAP exposure are likely. Furthermore, to examine the association between the snPCR results of the MAP-DNA occurrence in faeces, blood and semen, the chi-square value and the corresponding Phi-coefficient were calculated. These analyses could not be performed for semen and blood or semen and faeces because in both cases the ascertained frequencies were below the required frequencies. The statistical analysis between faeces and blood suggested weak associations. However, it should be mentioned that the associations were based on the degree of agreement of the positive as well as negative results and the calculated associations were mainly due to the degree of agreement of the negative results. For this reason, the results showed no connection between the circulation of MAP-DNA in blood and the simultaneous detection of MAP-DNA in faeces, as assumed. Furthermore, no statement can be made about the most appropriate matrix for MAP detection. In this study, MAP-DNA was detected most frequently (24.5\%) and also at highest concentrations of up to $6.25 \times 10^{6} \mathrm{MAP}-\mathrm{GE} / \mathrm{g}$ in faeces. However, in the longitudinal study of the bull's father published recently (Münster et al., 2013), MAPDNA was most frequently observed in blood samples (51.7\%), but with lower MAP$\mathrm{GE} / \mathrm{ml}$ of up to 1.79105 . In the present study, MAP-DNA was not detected in any of the three matrices (faeces, blood and semen) on 94 of 142 sampling days (66.2\%) and during intervals of up to 9 weeks (CW 12 to 20 in 2012). Furthermore, the commercial ELISA used did not show any antibody response throughout the whole investigation period of the bull. These results agree with a recent study, in which 30 of 33 cows were classified as MAP-free by three commercial serum ELISAs, although their MAP infection was previously confirmed by culture and PCR (Khol et al., 2012). Such negative ELISA results could be explained by the fact that ELISAs often used in the routine diagnostic of paratuberculosis are highly specific but lack in sensitivity for MAP-infected animals, especially in the subclinical stage of the disease. 
It is estimated that only $10 \%$ to $15 \%$ of cattle infected naturally with MAP will develop clinical symptoms. Most of them remain in the subclinical stage of the disease and excrete the pathogen intermittently (Olsen et al., 2002). The bull examined in our study was consistently clinically unremarkable during the entire study. At the time of necropsy, neither macroscopic nor histological findings consistent with a MAP infection or host reactions to MAP were observed, although PCR assays had shown a wide distribution of MAP-DNA in the host, including the tissues of the lymphatic system, digestive tract and urogenital tract. At first sight, such a macroscopic and histological picture in PCR-positive samples is unusual for a MAP infection. It is expected that in MAP-infected tissues, especially in the gastrointestinal tract, inflammatory processes would be present. In our study, the right tonsil was the only tissue where mycobacteria were proved by staining. However, these bacteria were interpreted as environmental mycobacteria because of negative MAP-PCR results and missing histological lesions. Extensive distribution of MAP in cattle tissues with no macroscopic lesions or clinical signs was also determined previously in cows (Brady et al., 2008). Moreover, other authors concluded that the distribution of MAP soon after the infection might be much more widespread in the host than commonly believed (Whitlock and Buergelt, 1996; Mortier et al., 2013). The distribution of MAP in organs distant from the gastrointestinal tract is different for each animal (Ayele et al., 2004). Here, MAP was not observed predominantly in the tissues of the bulls' gastrointestinal tract. If such uncommon distribution of MAP in the host depends on the pathway of the infection, it is not described yet in the literature.

Although it is generally accepted that MAP could be transmitted vertically from cow to foetus (Whittington and Windsor, 2009), information about the role of bulls in the distribution of MAP is rare. It has been argued that transmission of MAP via semen is unlikely due to the low amount of MAP in the ejaculates of infected bulls (Philpott, 1993; Buergelt and Williams, 2004). In the present study, MAP-DNA could also be determined in only four of 89 semen samples by snPCR and in only two of them detection of MAP-DNA was possible by qPCR, whereas quantification of MAP-GE failed due to the quantification limit. Cultivation of MAP colonies from this matrix was completely unsuccessful. Therefore, it is likely that the concentration of MAP was low in the investigated semen samples. However, Münster et al. (2013) demonstrated that MAPDNA was detectable repeatedly over a period of 3 years in concentrations of up to 
$5.7 \times 10^{5} \mathrm{MAP}-\mathrm{GE} / \mathrm{ml}$ in the semen of the bull's father, an also naturally infected, asymptomatic German Fleckvieh bull. In the present study, MAP-DNA was determined in the male reproductive organs of the bull after necropsy, including the testicles, epididymis, ampulla of deferent duct, prostate gland and superficial inguinal lymph nodes, the associated lymph nodes of the reproductive organs. In other studies, a wide distribution of MAP in the reproductive organs of bulls was also determined. But in contrast, these bulls showed clinical symptoms, or if they were asymptomatic, typical macroscopic abnormalities were found during necropsy, indicating an advanced stage of the disease (Larsen and Kopecky, 1970; Larsen et al., 1981; Ayele et al., 2004; Glawischnig et al., 2004; Khol et al., 2010). The question of whether or not semen can elicit an infection of the cow or foetus has not yet been investigated. If evidence did arise, it could be necessary to expand the breeding hygiene management to include MAP because of the direct and indirect contact of semen from a single bull with a high number of cows. Furthermore, infected bulls may represent a source of infection for other breeding bulls as it was shown for semen production units from Pakistan that $20.0 \%(47 / 235)$ of the breeding bulls and $33.3 \%$ (6/18) of the teaser bulls were infected with MAP by ELISA (Abbas et al., 2011).

In animals with clinical signs of paratuberculosis, the specificity of faecal culture is thought to reach $98 \%$ and the sensitivity of faecal culture is considered to reach $70 \%$. In contrast, cultivation of MAP from subclinically infected animals is difficult, because the sensitivity decreases to 23-49\%, and depends heavily on MAP shedding in faeces (Nielsen and Toft, 2008). In the present study, MAP growth was only found in three of 139 culturally investigated faecal and in two of 109 culturally investigated blood samples, over a period of 4 years. No MAP growth was observed in any of the bull's tissue samples and as mentioned before in semen samples. The longitudinal study of its infected but asymptomatic father showed also a great discrepancy between MAP detection by culture and PCR (Münster et al., 2013). Although in some samples of the investigated bull, high MAP-GE concentrations of up to $1.81 \times 10^{5} / \mathrm{ml}, 6.25 \times 10^{6} / \mathrm{g}$ and $3.86910^{6} / \mathrm{g}$ in blood, faecal and tissue samples, respectively, were measured by qPCR, the results in general show a low MAP content in the investigated samples because only a low number of the aliquots per sample were tested positive by snPCR. Additionally, this indicates an inhomogeneous distribution of MAP by formation of clusters. Also in a study that monitored a Limousin cattle herd for a period of 53 months, in a significantly 
high percentage of cattle, MAP was detectable by an IS900 qPCR but not in culture, respectively (Kralik et al., 2014); especially in the case of rather small numbers of MAP, the loss of viability of the bacteria during sample decontamination may reduce the detectability of MAP in culture (Bradner et al., 2013). In a previous study, the sensitivity of culture was estimated with approximately $10^{3} \mathrm{MAP}$ cells/g faeces (Kralik et al., 2011). Besides diagnostic measurement uncertainties, this discrepancy could be due to existence of dormant bacteria. Microorganisms in this state failed to grow by standard bacteriological methods, but remained alive (Oliver, 2010). MAP has the ability to enter into a viable but non-cultivable (VBNC) state in the environment (Whittington et al., 2004) and under experimental cultivation conditions (Lamont et al., 2012). Further, MAP-related bacteria such as Mycobacterium smegmatis and Mycobacterium tuberculosis can enter the VBNC state, too. For Mycobacterium tuberculosis, it could be demonstrated that, depending on the growth-stimulating factor in the sputum of infected patients, between 80 and $99.99 \%$ of the bacteria were in the VBNC stage (Mukamolova et al., 2010). Also, a recent study indicated that VBNC Mycobacterium tuberculosis bacteria persist within the CD271 ${ }^{+} / \mathrm{CD} 45^{-}$mesenchymal stem cells of the bone marrow, which provide a niche for dormant infection (Das et al., 2013) and seem to play a key role in the establishment of latent tuberculosis (Raghuvanshi et al., 2010). In the investigated bull, it was striking that MAP-DNA was also detected in a high number of the analysed bone marrow aliquots (9/12) by snPCR. The missing cultivation of MAP from these samples may also suggest the VBNC state.

\section{Acknowledgements}

This work was funded by the Federal Ministry of Economics and Technology under support code KF2484201AJ9. The authors would like to thank Jürgen Rehage (Clinic for Cattle, University of Veterinary Medicine Hannover, Germany) for the collection of the biopsy samples by routine endoscopy. We are grateful to Elisabeth M. LieblerTenorio (Institute of Molecular Pathogenesis, Federal Research Institute for Animal Health, Friedrich- Loeffler-Institute, Jena, Germany) for performing the histopathology and immunohistology investigations in tissue samples. The authors wish to thank Melissa Kivoloka, Tina Althaus and Simone Urstadt for their assistance in sample processing. We are grateful to Lynne Riddles for completing the critical review of the manuscript. 


\section{CHAPTER I}

\section{Conflict of Interest}

The authors do not have a personal or financial relationship with other people or organizations that could inappropriately influence or bias this paper. All authors have approved the paper for release and are in agreement with its content.

\section{References}

Abbas, M., M. Munir, S. A. Khaliq, M. I. Haq, M. Tanveer Khan and Z. U. Qureshi, 2011: Detection of paratuberculosis in breeding bulls at pakistani semen production units: a continuous source of threat. ISRN veterinary science, 2011, 501235 .

Ayele, W. Y., M. Bartos, P. Svastova and I. Pavlik, 2004: Distribution of Mycobacterium avium subsp. paratuberculosis in organs of naturally infected bull-calves and breeding bulls. Veterinary microbiology, 103, 209-217.

Bradner, L., S. Robbe-Austerman, D. C. Beitz and J. R. Stabel, 2013: Optimization of hexadecylpyridinium chloride decontamination for culture of Mycobacterium avium subsp. paratuberculosis from milk. Journal of clinical microbiology, 51, 1575-1577.

Brady, C., D. O'Grady, F. O'Meara, J. Egan and H. Bassett, 2008: Relationships between clinical signs, pathological changes and tissue distribution of Mycobacterium avium subspecies paratuberculosis in 21 cows from herds affected by Johne's disease. The Veterinary record, 162, 147-152.

Buergelt, C. D. and J. E. Williams, 2004: Nested PCR on blood and milk for the detection of Mycobacterium avium subsp paratuberculosis DNA in clinical and subclinical bovine paratuberculosis. Australian veterinary journal, 82, 497-503.

Collins, M. T. and B. Y. Zhao, 1994: Comparison of the commercial serum antibody ELISA, -interferon test kit, and radiometric fecal culture for early diagnosis of paratuberculosis in experimentally infected Holstein calves. In: Chiodini, R.J., Collins, M.T., Bassey, E.O.E. (Eds.), Proceedings of the Fourth International Colloquium on Paratuberculosis. Evergreen Press, East Providence, pp. 67-76. 
Czerny, C. P. and P. Münster, 2013: Detection method for Mycobacterium avium ssp. paratuberculosis. Georg-August-University Göttingen Public Law Foundation. Patent WO 2013160434. 2013 Okt 31.

Das, B., S. S. Kashino, I. Pulu, D. Kalita, V. Swami, H. Yeger, D. W. Felsher and A. Campos-Neto, 2013: CD271(+) bone marrow mesenchymal stem cells may provide a niche for dormant Mycobacterium tuberculosis. Science translational medicine, 5, 170ra113.

Eisenberg, S. W., A. P. Koets, M. Nielen, D. Heederik, R. Mortier, J. De Buck and K. Orsel, 2011: Intestinal infection following aerosol challenge of calves with Mycobacterium avium subspecies paratuberculosis. Veterinary research, 42, 117.

Friedrich-Loeffler-Institut, 2014: Official Manual of Diagnostic Procedures. Available at: https://openagrar.bmelforschung.de/servlets/MCRFileNodeServlet/Document_derivate_00005327/TK18 _Paratuberkulose_2014929.pdf;jsessionid=3308B9CA153B08F3548E5483D409 A41C (accessed Marc 2014).

Glawischnig, W., M. Awad-Masalmeh, D. Khaschabi and M. Schonbauer, 2004: Detection of Mycobacterium avium subsp. paratuberculosis from the testicles of a clinically infected breeding animal. Berliner und Münchener tierärztliche Wochenschrift, 117, 136-139.

Hasonova L., Trcka I., Babak V., Rozsypalova Z., Pribylova R. and Pavlik I., 2009: Distribution of Mycobacterium avium subsp paratuberculosis in tissues of naturally infected cattle as affected by age. Veterinarni Medicina, 54, 257-269.

Khol, J. L., E. Geisbauer, M. Wassertheurer, S. Revilla-Fernandez, J. Damoser, E. Osterreicher, M. Dunser, U. Kleb and W. Baumgartner, 2012: Outcome of three commercial serum ELISAs and faecal detection of Mycobacterium avium subsp. paratuberculosis in consecutive samples from a cattle herd with low prevalence of paratuberculosis (Johne's disease). Transboundary and emerging diseases, 59, 197-207.

Khol, J. L., P. Kralik, I. Slana, V. Beran, C. Aurich, W. Baumgartner and I. Pavlik, 2010: Consecutive excretion of Mycobacterium avium subspecies paratuberculosis in semen of a breeding bull compared to the distribution in 
feces, tissue and blood by IS900 and F57 quantitative real-time PCR and culture examinations. The Journal of veterinary medical science / the Japanese Society of Veterinary Science, 72, 1283-1288.

Kralik, P., R. Pribylova-Dziedzinska, A. Kralova, K. Kovarcik and I. Slana, 2014: Evidence of passive faecal shedding of Mycobacterium avium subsp. paratuberculosis in a Limousin cattle herd. Veterinary journal, 201, 91-94.

Kralik, P., I. Slana, A. Kralova, V. Babak, R. H. Whitlock and I. Pavlik, 2011: Development of a predictive model for detection of Mycobacterium avium subsp. paratuberculosis in faeces by quantitative real time PCR. Veterinary microbiology, 149, 133-138.

Krüger, C., H. Köhler and E. M. Liebler-Tenorio, 2015: Sequential development of lesions 3, 6, 9, and 12 months after experimental infection of goat kids with Mycobacterium avium subsp paratuberculosis. Veterinary pathology, 52, 276290.

Lambeth, C., L. A. Reddacliff, P. Windsor, K. A. Abbott, H. McGregor and R. J. Whittington, 2004: Intrauterine and transmammary transmission of Mycobacterium avium subsp paratuberculosis in sheep. Australian veterinary journal, 82, 504-508.

Lamont, E. A., J. P. Bannantine, A. Armien, D. S. Ariyakumar and S. Sreevatsan, 2012: Identification and characterization of a spore-like morphotype in chronically starved Mycobacterium avium subsp. paratuberculosis cultures. PloS one, 7, e30648.

Larsen, A. B. and K. E. Kopecky, 1970: Mycobacterium paratuberculosis in reproductive organs and semen of bulls. American journal of veterinary research, $31,255-258$.

Larsen, A. B., O. H. Stalheim, D. E. Hughes, L. H. Appell, W. D. Richards and E. M. Himes, 1981: Mycobacterium paratuberculosis in the semen and genital organs of a semen-donor bull. Journal of the American Veterinary Medical Association, $179,169-171$.

Li, L., J. P. Bannantine, Q. Zhang, A. Amonsin, B. J. May, D. Alt, N. Banerji, S. Kanjilal, and V. Kapur, 2005: The complete genome sequence of Mycobacterium 
avium subspecies paratuberculosis. Proceedings of the National Academy of Sciences of the United States of America 102, 12344-12349.

Littell, R. C., G. A. Milliken, W. W. Stroup and R. D. Wolfinger, 1999: SAS system for mixed model. SAS Inst., Cary, NC.

McDonald, W. L., S. E. Ridge, A. F. Hope and R. J. Condron, 1999: Evaluation of diagnostic tests for Johne's disease in young cattle. Australian veterinary journal, 77, 113-119.

Mortier, R. A., H. W. Barkema, J. M. Bystrom, O. Illanes, K. Orsel, R. Wolf, G. Atkins and J. De Buck, 2013: Evaluation of age-dependent susceptibility in calves infected with two doses of Mycobacterium avium subspecies paratuberculosis using pathology and tissue culture. Veterinary research, 44, 94.

Mukamolova, G. V., O. Turapov, J. Malkin, G. Woltmann and M. R. Barer, 2010: Resuscitation-promoting factors reveal an occult population of tubercle Bacilli in Sputum. American journal of respiratory and critical care medicine, 181, 174180.

Münster, P., I. Völkel, W. Wemheuer, J. Petschenka, W. Wemheuer, C. Steinbrunn, A. Campe, W. J. Schulz-Schaeffer, L. Kreienbrock and C. P. Czerny, 2011: Detection of Mycobacterium avium ssp. paratuberculosis in ileocaecal lymph nodes collected from elderly slaughter cows using a semi-nested IS900 polymerase chain reaction. Veterinary microbiology, 154, 197-201.

Münster, P., I. Völkel, W. Wemheuer, D. Schwarz, S. Doring and C. P. Czerny, 2013: A longitudinal study to characterize the distribution patterns of Mycobacterium avium ssp. paratuberculosis in semen, blood and faeces of a naturally infected bull by IS 900 semi-nested and quantitative real-time PCR. Transboundary and emerging diseases, 60, 175-187.

Nielsen, S. S. and N. Toft, 2008: Ante mortem diagnosis of paratuberculosis: a review of accuracies of ELISA, interferon-gamma assay and faecal culture techniques. Veterinary microbiology, 129, 217-235.

Oliver, J. D., 2010: Recent findings on the viable but nonculturable state in pathogenic bacteria. FEMS microbiology reviews, 34, 415-425. 
Olsen, I., G. Sigurgardottir and B. Djonne, 2002: Paratuberculosis with special reference to cattle. A review. The Veterinary quarterly, 24, 12-28.

Philpott, M., 1993: The dangers of disease transmission by artificial insemination and embryo transfer. The British veterinary journal, 149, 339-369.

Pribylova, R., I. Slana, S. Cech, A. Kralova and I. Pavlik, 2013: Mycobacterium avium subsp. paratuberculosis detected in the reproductive tract of cows from an infected herd. Reproduction in domestic animals = Zuchthygiene, 48, 790-794.

Raghuvanshi, S., P. Sharma, S. Singh, L. Van Kaer and G. Das, 2010: Mycobacterium tuberculosis evades host immunity by recruiting mesenchymal stem cells. Proceedings of the National Academy of Sciences of the United States of America, 107, 21653-21658.

Raizman, E. A., J. P. Fetrow and S. J. Wells, 2009: Loss of income from cows shedding Mycobacterium avium subspecies paratuberculosis prior to calving compared with cows not shedding the organism on two Minnesota dairy farms. Journal of dairy science, 92, 4929-4936.

Streeter, R. N., G. F. Hoffsis, S. Bech-Nielsen, W. P. Shulaw and D. M. Rings, 1995: Isolation of Mycobacterium paratuberculosis from colostrum and milk of subclinically infected cows. American journal of veterinary research, 56, 13221324.

Taylor, T. K., C. R. Wilks and D. S. McQueen, 1981: Isolation of Mycobacterium paratuberculosis from the milk of a cow with Johne's disease. The Veterinary record, 109, 532-533.

van Roermund, H. J., D. Bakker, P. T. Willemsen and M. C. de Jong, 2007: Horizontal transmission of Mycobacterium avium subsp. paratuberculosis in cattle in an experimental setting: calves can transmit the infection to other calves. Veterinary microbiology, 122, 270-279.

Whitlock, R. H. and C. Buergelt, 1996: Preclinical and clinical manifestations of paratuberculosis (including pathology). The Veterinary clinics of North America. Food animal practice, 12, 345-356. 


\section{CHAPTER I}

Whittington, R. J., D. J. Marshall, P. J. Nicholls, I. B. Marsh and L. A. Reddacliff, 2004: Survival and dormancy of Mycobacterium avium subsp. paratuberculosis in the environment. Applied and environmental microbiology, 70, 2989-3004.

Whittington, R. J. and P. A. Windsor, 2009: In utero infection of cattle with Mycobacterium avium subsp. paratuberculosis: a critical review and metaanalysis. Veterinary journal, 179, 60-69. 


\section{CHAPTER II}

\section{Paper published}

Journal of Medical Primatology

\section{Detection of Mycobacterium avium subsp. paratuberculosis by IS900 based PCR assays in non-human primates kept in Germany}

Kim Fechner ${ }^{\mathrm{a}}$, Kerstin Mätz-Rensing ${ }^{\mathrm{b}}$, Karen Lampe ${ }^{\mathrm{b}}$, Franz-Josef Kaup ${ }^{\mathrm{b}}$, Claus-Peter Czerny $^{\mathrm{a}}$ and Jenny Schäfer ${ }^{\mathrm{a}^{*}}$

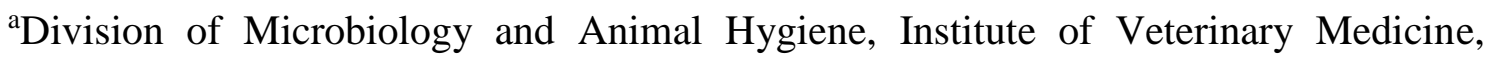
Department of Animal Sciences, Faculty of Agricultural Sciences, Georg-AugustUniversity Göttingen, Burckhardtweg 2, D-37077 Göttingen, Germany.

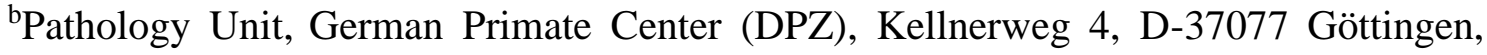
Germany.

*Corresponding author. Mailing address: Department of Animal Sciences, GeorgAugust-University Göttingen, Burckhardtweg 2, D-37077 Göttingen, Germany. Phone: +49 55139 13855. Fax: +49 55139 33035. E-mail: jschaef@ gwdg.de 


\section{Abstract}

Background Due to a sporadic occurrence of Mycobacterium avium subsp. paratuberculosis (MAP) in non-human primates (NHP), the susceptibility of different NHP to MAP should be investigated.

Methods Fecal and tissue samples (ileum, ileocecal lymph nodes, bone marrow) of 20 animals (seven species) were analyzed by IS900-based PCRs and sequenced. Samples of MAP PCR positive NHP were further cultivated.

Results MAP DNA was detectable in two animals; the ileum of a cottontop tamarin and the bone marrow of a common marmoset. Cultivation of MAP failed. Sequence analysis revealed $100 \%$ homology to the MAP-K10 sequence. Pathohistological examinations offered no direct correlation to a MAP infection.

Conclusions MAP was detected for the first time in a common marmoset. But as both NHP suffered from other diseases an asymptomatic infection with MAP was assumed. The detection of MAP in the bone marrow might play a role in establishing latent paratuberculosis, as known from tuberculosis.

Keywords: bacteriological infection; bone marrow; Callithrix jacchus; Saguinus oedipus; PCR 


\section{Introduction}

Non-human primates are susceptible to acute or chronic enteral infections with different mycobacteria. One common form of enteritis, so called John's disease or paratuberculosis is induced by Mycobacterium avium subsp. paratuberculosis (MAP). In general, this disease occurs in ruminants, but pathological and histological changes typical for paratuberculosis have also been reported in several non-ruminant species, such as rabbits, donkeys and non-human primates [1-4]. Characteristic for the pathological picture of paratuberculosis is a thickened intestinal mucosa with corrugation and an enlargement of regional lymph nodes. Histopathologically, paratuberculosis is characterized by a chronic progressive granulomatous inflammation of the intestinal tract. The reasons for death, is the development of profuse diarrhea accompany with proteinemia, causing dehydration and cachexia [5]. Such typical clinical symptoms for paratuberculosis were observed previously in two non-human primate species [2-3]. MAP was detected in different tissues, particularly in the gastrointestinal tract of a captive group of stumptail macaques (Macaca arctoides) [2] and a captive female mandrill (Mandrillus shinx) [3]. Furthermore, the pathogen was detected in 10 out of $25(40 \%)$ or 2 of $25(8 \%)$ fecal samples of rhesus macaques (Macaca mulatta) by microscopic examination or direct IS900-based PCR, respectively [6]. These 25 fecal samples were collected from six free-living colonies with multiple clinical conditions (coughing and stool loose) from North India. The presence of MAP in non-human primates, which are kept in a German zoological garden, was previously shown [7]. The authors detected MAP DNA in each pooled fecal sample from 3 different non-human primate species; cottontop tamarins (Saguinus oedipus), gelada baboons (Theropithecus gelada), and black-and-white ruffed lemurs (Varecia variegata). Genome equivalents between $2.2 \times 10^{3}$ and $9.6 \times 10^{6} \mathrm{MAP} / \mathrm{g}$ feces were measured using MAP-specific PCRs. Ascertained MAP genome equivalent concentrations of black-and-white ruffed lemurs were highest in comparison to other examined animal species, such as goats, cavies, parrots or antelopes. Nevertheless, the detection of MAP in feces does not allow for a statement about a true infection with active shedding of MAP in feces or a pass through with passive MAP shedding in feces only $[8,9]$. An infection with MAP is given when the pathogen exceeds the intestinal mucosa barrier and is distributed within the local tissue or is transported via the blood and lymphatic system to other areas of the body [10]. This study aims to demonstrate a 
possible MAP infection in different non-human primate species by comparable investigations of feces and tissue samples using two MAP-specific PCRs and culture.

\section{Material and methods}

\section{Humans care guidelines}

Care and housing conditions of the animals, kept at the German Primate Center (DPZ), complied with the regulations of the European Parliament and the Council Directive on the protection of animals used for scientific purposes (2010/63/EU) and the National Institutes of Health Guide for the Care and Use of Laboratory Animals (2010). The animals in the zoological gardens are kept in accordance with the guidelines of the European Union for the accommodation and care of wild animals in zoological gardens (1999/22/EG). Furthermore, all primate facilities follow the applicable German Animal Protection Law. The investigated non-human primates from the DPZ were involved in different experimental studies. All examined animals suffered from severe diseases or were euthanized after study end. For the present study, only leftovers were used.

\section{Animals and sampling}

A total of 20 non-human primates belonging to seven different species were examined (Table 1). Four animals lived in different German zoological gardens; the other 16 nonhuman primates were kept at the DPZ. Altogether, the investigated species included one cottontop tamarin (Saguinus oedipus; No. 1), one emperor tamarin (Saguinus imperator; No. 2), eight common marmosets (Callithrix jacchus; No. 3 to 10), one barbary macaque (Macaca sylvanus; No. 11), one long-tailed macaque (Macaca fascicularis; No. 12), seven rhesus macaques (Macaca mulatta; No. 13 to 19) and one guereza (Colobus guereza; No. 20). During necropsy, tissue samples from the ileum, ileocecal lymph nodes, bone marrow, as well as fecal samples, were collected under sterile conditions and were transferred in sterile collection tubes by professional veterinary staff at the DPZ. All samples were analyzed directly or stored at $-20^{\circ} \mathrm{C}$ prior to the respective assays. To avoid fecal contaminations, the ileum was cleaned thoroughly by flushing with sterile PBS before examination. 


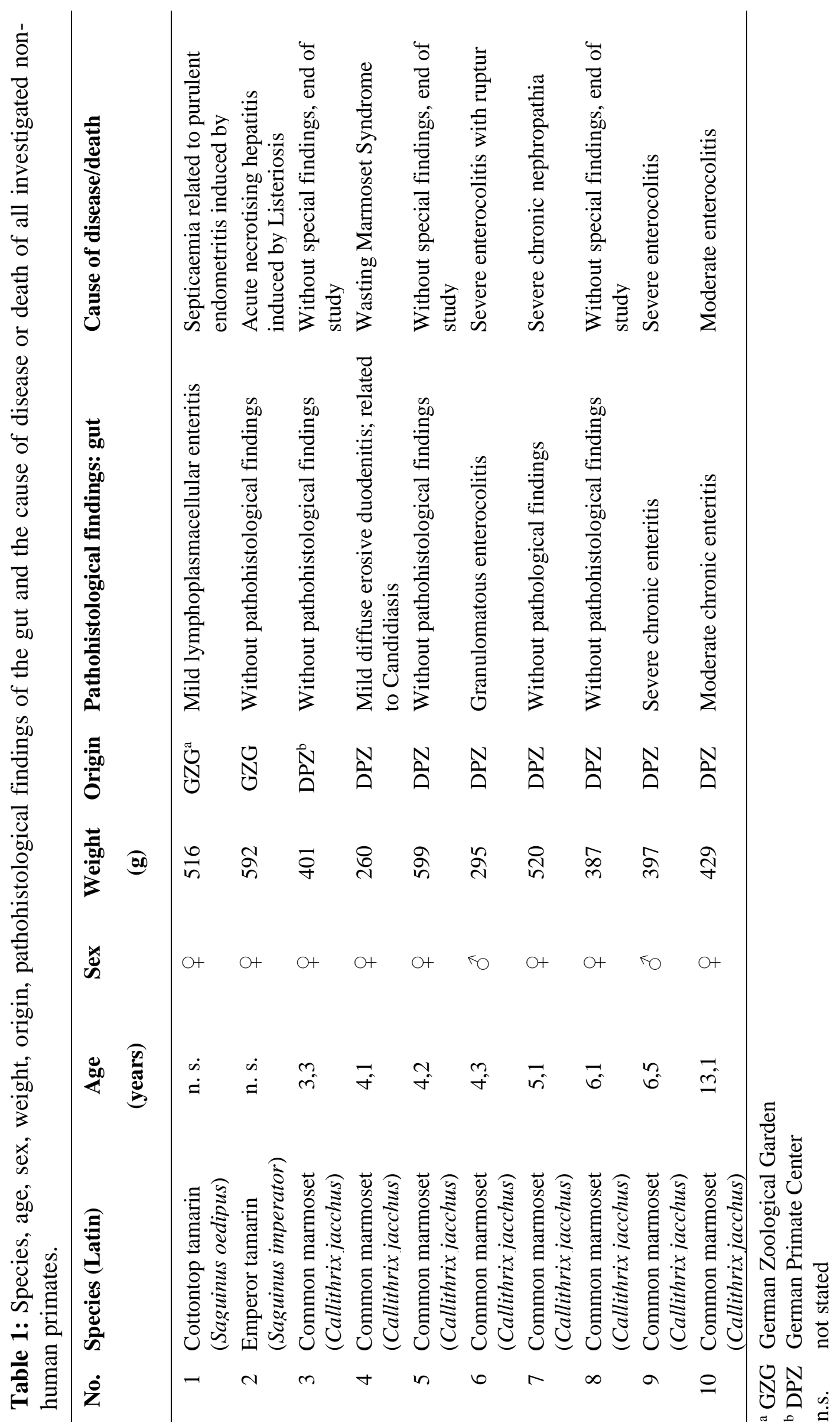




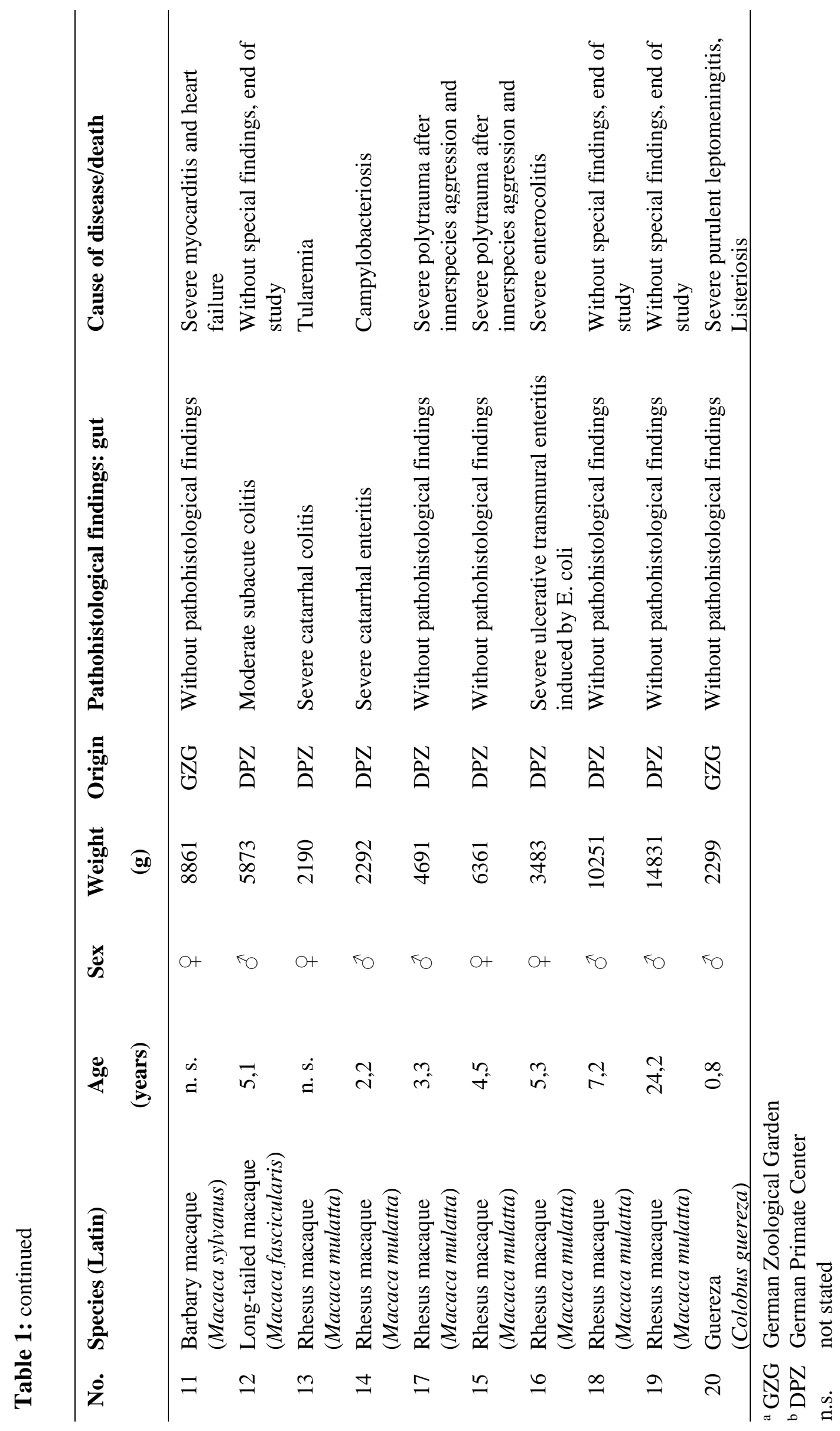




\section{DNA extraction}

DNA extraction and purification of all collected fecal and tissue samples was performed using a modified protocol of the QIAamp Blood Kit and QIAamp DNA Mini Kit (Qiagen, Hilden, Germany), respectively. Two aliquots of $50 \mathrm{mg}$ of the sample matrices were transferred to $2 \mathrm{ml}$ Precellys ${ }^{\circledR}$ tubes (Precellys ${ }^{\circledR}$ Ceramic Kit 1.4, $2.8 \mathrm{~mm}$, Peqlab, Erlangen, Germany) containing $160 \mu 15 \%$ (w/v) SDS solution. Fecal and tissue samples were homogenized for $15 \mathrm{sec}$ at $6000 \mathrm{rpm}$ and $30 \mathrm{sec}$ at $6500 \mathrm{rpm}$ in a Precellys ${ }^{\circledR} 24$ homogenizer (Peqlab, Erlangen, Germany), respectively. Homogenized samples were incubated in a thermomixer at $37^{\circ} \mathrm{C}$ and at $900 \mathrm{rpm}$ for $30 \mathrm{~min}$. Further purification of the tissue samples was performed, as described elsewhere [11]. To the homogenized fecal samples $400 \mu \mathrm{l}$ lysis buffer (AL buffer) and $40 \mu \mathrm{l}$ ready-to-use proteinase $\mathrm{K}$ solution $(20 \mathrm{mg} / \mathrm{ml})$ were added. Mixed samples were incubated in a thermomixer at $56^{\circ} \mathrm{C}$ and at $900 \mathrm{rpm}$ for $30 \mathrm{~min}$, followed by a further $15 \mathrm{~min}$ at $95^{\circ} \mathrm{C}$. After centrifugation $(20000 \times \mathrm{g}, 1 \mathrm{~min})$, the supernatants were transferred into new tubes before $400 \mu$ l ethanol ( $\geq 99.8 \%$ ) was added. Loading of the QIAamp Spin Column and washing procedures were performed according to the manufacturer's instructions. Finally, the DNA of the fecal and tissue samples was eluted in $100 \mu 1$ elution buffer at $85^{\circ} \mathrm{C}$, and stored at $-20^{\circ} \mathrm{C}$ prior to use. To ensure contamination-free DNA preparation, a negative control was included during each DNA extraction by adding $50 \mu 1$ sterile distilled water instead of the sample matrix.

\section{Qualitative and quantitative IS900 polymerase chain reactions}

Qualitative semi-nested PCR (snPCR) and quantitative real-time PCR (qPCR) were performed as described elsewhere [11, 12]. Amplification of DNA by snPCR resulted in fragment lengths of $587 \mathrm{bp}$ and $278 \mathrm{bp}$, by qPCR in $139 \mathrm{bp}$, respectively. MAP DNA, extracted from the MAP reference strain ATCC 19698 (Leibnitz Institute DSMZ, German Collection of Microorganisms and Cell Cultures, Braunschweig, Germany), was included as a positive control in each PCR run, whereas sterile distilled water was used as a negative control. MAP genome equivalents (MAP-GE) detected by qPCR were calculated on the base of the MAP K-10 genome size (4.829.781 bp) [13]. One MAP-GE is calculated about 5.29 fg of MAP-DNA, assuming that the molecular weight of one base pair is $660 \mathrm{~g} \times \mathrm{mol}^{-1}$ and the Avogadro number is $6.022 \times 10^{23} \mathrm{~mol}^{-1}$. The number of MAP-GE in fecal or tissue sample per $g$ were calculated by the following formula: Concentration of DNA $(\mathrm{fg} / \mu \mathrm{l}) \times$ volume of eluted DNA $(50 \mu \mathrm{l}) / 5.29 \mathrm{fg} \times 20$ 
$(50 \mathrm{mg}$ fecal or tissue sample $)=$ MAP-GE per extracted sample aliquot, per $\mathrm{ml}$ or $\mathrm{g}$ sample, respectively. The detection limit of the snPCR was $2.5 \mathrm{fg}$ MAP DNA/reaction (approximately 1 genome equivalents/reaction) [14, 15], whereas the qPCR had a detection limit of $50 \mathrm{fg} /$ reaction (approximately 10 MAP genome equivalents/reaction) and a quantification limit of $5 \mathrm{pg}$ MAP DNA/reaction (approximately 950 MAP genome equivalents/reaction) $[11,12]$. Due to the lower sensitivity of the qPCR, only aliquots that tested positive for MAP by snPCR were further analyzed by qPCR in three independent qPCR runs.

\section{Cloning and sequencing of partial IS900 sequences}

The $587 \mathrm{bp}$ fragments obtained from snPCR, covering nucleotide positions 183 to 770 within the IS900, were cloned into the StrataClone ${ }^{\mathrm{TM}}$ PCR Cloning Vector and transformed into E. coli, according to the manufacturer's instructions (Agilent Technologies, Waldbronn, Germany). Afterwards, plasmid DNA was extracted from two clones per amplicon using QIAprep ${ }^{\circledR}$ Spin Miniprep Kit (Qiagen, Hilden, Germany). Sequencing of plasmid inserts (both strand directions of each clone) was conducted by the Göttingen Genomics Laboratory (Germany). Sequences were compared to all sequences from GenBank entries (www.ncbi.nlm.nih.gov) using BLAST search. To monitor similarities within all generated clone sequences, an alignment with published IS900 sequences was performed using the sequence analysis software “MegAlign”' (DNA Star, Inc., Wisconsin, USA).

\section{Pathohistological investigations}

Samples for histopathology were collected during necropsies of animals sent for routine diagnostic to the pathology laboratory. Tissue samples were immersion fixed in $10 \%$ neutral buffered formalin. Tissues underwent routine histological processing. They were embedded in paraffin. Sections were stained with standard eosin-hematoxylin (HE) using the Varistain Gemini staining machine (Thermo Fisher, Scientific, Frankfurt/Main, Germany). Selected sections from the gastrointestinal tract were stained with Ziehl-Neelsen and Fite Faraco Kynjon using standardized protocols to demonstrate acid fast bacteria. 


\section{Bacteriological investigations for MAP}

All four sample matrices (ileum, ileocecal lymph nodes, bone marrow and feces) from the non-human primates, which tested positive for MAP by PCR, were used for MAP cultivation. About $500 \mathrm{mg}$ of feces and a maximum of $150 \mathrm{mg}$ of tissue material could be prepared due to the small size of the new world monkeys. The procedure was carried out according to the official German manual of diagnostic procedures [16] using adapted amounts of reagents in relation to the sample volumes, and the tissue homogenization method was modified. Tissues were transferred to $2 \mathrm{ml}$ Precellys ${ }^{\circledR}$ tubes (Precellys ${ }^{\circledR}$ Ceramic Kit 1.4, $2.8 \mathrm{~mm}$, Peqlab, Erlangen, Germany) containing 150 $\mu 1$ PBS. All tissue aliquots were homogenized for $30 \mathrm{sec}$ at $6000 \mathrm{rpm}$ in a Precellys ${ }^{\circledR} 24$ homogenizer. Fecal samples were homogenized by thorough vortexing. For decontamination purposes, the fecal and tissue homogenates were suspended in $5 \mathrm{ml}$ of $0.75 \%$ hexadecylpyridinium chloride (HPC) solution. Further preparation steps were taken as described elsewhere $[11,16]$. MAP cultures were performed in parallel on two tubescolumns of Herrold's egg yolk medium (HEYM, BD BBL ${ }^{\mathrm{TM}}$, Heidelberg, Germany) and in one modified Middlebrook 7H9 broth medium (M7H9C) [17]. In addition, negative as well as positive control fecal and tissue samples, mixed with the MAP reference strain ATCC 19698, were analyzed. Growths of bacterial cultures were monitored for the first time after four weeks and afterwards regularly every 4 weeks for up to 20 weeks. Cultures exhibiting suspicious colonies were verified by the abovementioned snPCR.

\section{Results}

\section{Detection and quantification of MAP DNA by PCRs}

In 2 out of the $20(10 \%)$ investigated non-human primates, MAP DNA was detected by a MAP-specific IS900 based snPCR (Fig. 1). Specifically, MAP DNA was detected in the ileum of a female cottontop tamarin (Saguinus oedipus; No. 1) and in the bone marrow of a female common marmoset (Callithrix jacchus; No. 4). Both tissues also tested positive for MAP DNA by qPCR, but quantification failed, due to an amount below the quantification limit of 950 genome equivalents/reaction. 


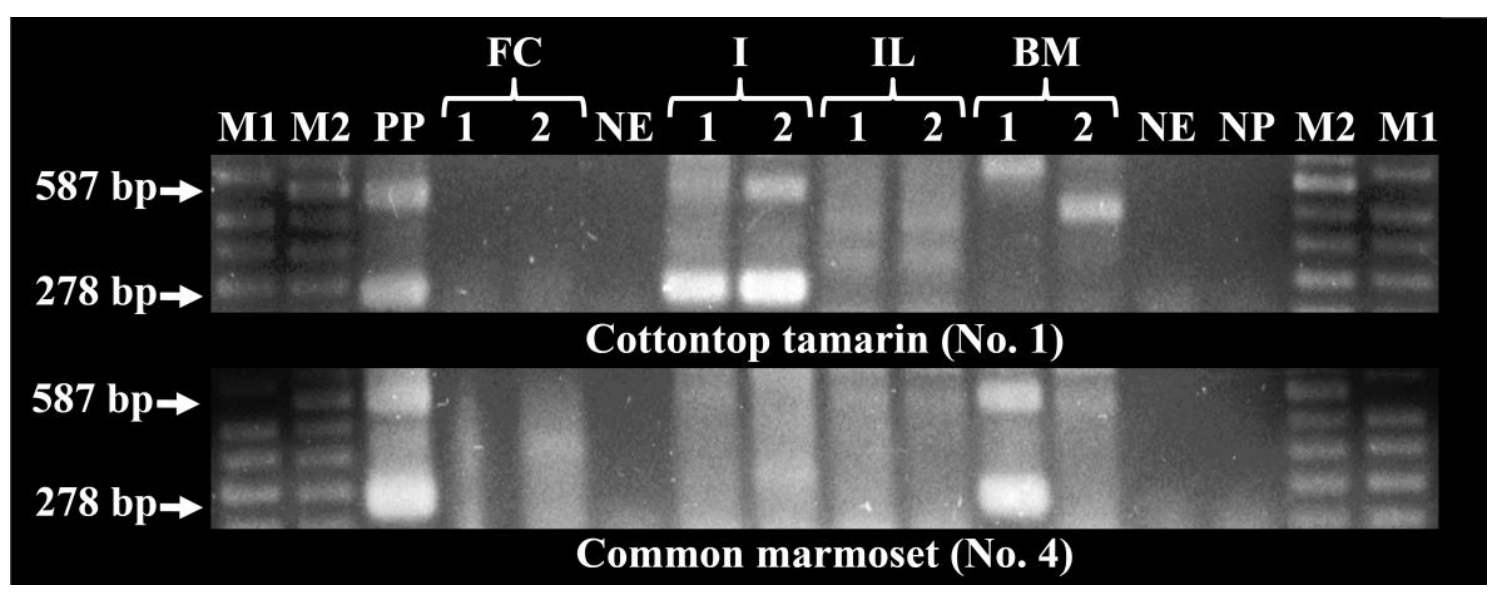

Figure 1: Detection of MAP DNA in a female cottontop tamarin (Saguinus oedipus; No. 1) and in a female common marmoset (Callithrix jacchus; No. 4) by snPCR, visualized by gel electrophoresis. Depending on the MAP DNA concentration, either the specific $278 \mathrm{bp}$ fragment alone or the two $278 \mathrm{bp}$ and $587 \mathrm{bp}$ fragments were amplified and were responsible for a positive validation. M1, $1 \mathrm{~kb}$ molecular weight marker; M2, 100 bp molecular weight marker; PP, positive control of PCR (MAPDNA); 1 and 2, each both PCR aliquots of sample matric; FC, fecal sample; I, ileum sample; IL, ileocecal lymph nodes sample; BM, bone marrow sample; NE, negative control of the DNA extraction; NP, negative control of PCR (sterile distilled water).

\section{Sequencing of partial IS900 sequences}

To confirm the specificity of the snPCR and in order to detect polymorphisms within the partial IS900 sequence, the 587bp DNA fragments from both samples, which tested positive by snPCR, were sequenced. A comparison between the analyzed sequences from the ileum of the cottontop tamarin (No.1) and the analyzed sequences from the bone marrow of the common marmoset (No.4) revealed a homology degree of $100 \%$ with the published bovine MAP-K10 IS900 reference sequence (GenBank: AE016958).

\section{Pathological and histological investigations}

The main pathological and histological findings of the gut and the cause of disease or death for all investigated non-human primates are listed in Table 1. In general, no direct correlation between MAP and the individual cause of disease or death exists. Nevertheless, the pathological and histological findings of both non-human primates, which tested positive for MAP by PCR, were further described in detail. The cottontop 
tamarin (No. 1) was found unconscious in a German zoological garden. The primate had a good nutritional condition. Main pathological and histological findings were observed in the reproductive system. The cause of disease and death was an acute purulent endometritis induced by a staphylococcus infection and followed by sepsis. Interestingly, mild, mainly plasmacellular duodenitis and ileitis were found in the small intestine. The mesenteric lymph nodes were also activated. Both were secondary findings unrelated to the disease. Acid fast bacteria were not demonstrable within the mucosa. Therefore, a direct correlation between MAP and the mild chronic enteritis remains questionable.

The common marmoset (No. 4) belonged to a group of primates from the DPZ and was euthanized due to its progressive weight loss. Pathologically striking were findings associated with the Marmoset Wastin Syndrome. Histologically, a mild erosive duodenitis was found, which was accompanied by the microbiological detection of Candida, as well as a mild cholecystitis.

\section{Bacteriological investigations for MAP}

In all culturally investigated matrices (ileum, ileocecal lymph nodes, bone marrow and feces) of both non-human primates, which tested positive for MAP by PCR, growth of MAP could not be observed after 20 weeks, neither on HEYM nor in M7H9C.

\section{Discussion}

A few studies have shown so far that MAP could be observed in non-human primates $[2,3,6,7]$. The first evidence for the presence of MAP in non-human primates kept in Germany was previously described in a screening study of pooled fecal samples from a zoological garden [7]. However, the detection of MAP in fecal samples could not confirm an infection, due to the fact that passive MAP shedding is possible. In this study, it was demonstrated that cottontop tamarins not only excrete MAP via feces [7], they are actually infected with MAP due to the detection of MAP DNA in the ileum. In rhesus macaques, neither an infection with MAP nor an excretion of MAP via feces could be shown in seven investigated animals, although MAP was already detected in feces of rhesus macaques previously [6]. Nevertheless, an infection of a common marmoset is demonstrated in this study for the first time due to the detection of MAP 
DNA in the bone marrow. These findings indicate a prolonged spreading of MAP in different non-human primate species as known before $[2,3,6,7]$. Whether non-human primates were responsible for the infection of other animals or not - for example primates in colonies or animals in zoological facilities - is not clear, but the list of animals, which are potential sources for MAP transmission, has been expanded.

Nevertheless, the possible distribution of MAP via feces of non-human primates was not measurable as part of this study, even though it is known from literature that MAP can be excreted in concentrations of $10^{6} \mathrm{MAP}$ genome equivalent/g feces from asymptomatic non-humane primates [7]. Similar MAP concentrations were also measurable in feces of other animals asymptomatically infected but it is known that the excretion of MAP appears usually intermittently $[11,14,18]$. Hence, it is admitted that diagnostic tests may lead temporarily to negative results during shedding-free intervals of the animals. This could potentially be an explanation for negative results in the analyzed fecal samples, especially in the case of the two MAP PCR positive non-human primates. However, diagnostic uncertainties may also occur due to low numbers of MAP cells (lower than the detection limit of the used method), limited sample volume or inhomogeneous distribution of MAP within the samples. In this study, the presence of MAP DNA was demonstrated in 2 out of 20 (10\%) investigated non-human primates, but only in single matrices alone and by PCR analysis. Therefore, from the failure of quantification by qPCR it is obvious that the detectability of MAP was influenced by low numbers of MAP as well as low amounts of sample material.

Nevertheless, it is known that also other mycobacteria, like Mycobacterium simiae, could cause paratuberculosis similar symptoms in non-human primates [19]. To exclude an infection with mycobacteria other than MAP and to monitor the MAP types, which are involved in the current two cases, the detected MAP DNA was further characterized. PCR amplicons were sequenced to differentiate MAP strains by unique single nucleotide polymorphisms (SNPs) in specific regions of the IS900. It has been shown that SNPs at positions 169 (T/C) and 216 (G/A) are characteristic of MAP strains isolated from sheep in comparison to cattle types [20, 21]. The $587 \mathrm{bp}$ PCR product of the used snPCR covered the 216 position. Adenine peaks at these loci in the sequence of the PCR amplicons from the ileum of the cottontop tamarin and the bone marrow of the common marmoset gave the indication that the detected MAP sequences may belong to a cattle strain. Nevertheless, occasionally an adenine peak was also found in the sheep 
type of MAP. Literature suggests that all isolates belonging to the non-sheep type of MAP are highly conserved and thus cluster as a homogeneous group [22]. Moreover, also isolates from humans are thought to cluster with bovine strains [23].

In contrast to ruminants, studies reporting on monogastric animals with clinical symptoms and/or pathological and histological changes, associated with a MAP infection, are rare. Only two reports of non-human primates were found describing typical granulomatous enteritis induced by MAP [2,3]. With careful interpretation of histological and pathological findings, the determined low-grade inflammatory processes in the gastrointestinal tract and the activation of the lymphatic system in the investigated cottontop tamarin (No. 1) - in which MAP DNA was detected in the ileum - could be associated with a MAP infection. However, these changes were secondary findings unrelated to disease or the death of the animal. The pathological and histological changes observed in the common marmoset (No. 4) indicate an underlying Wasting Marmoset Syndrome (WMS), which could not be classified in detail. In this study, it was interesting that MAP DNA was detected in two marmoset species (Callitrichidae), since these species often develop chronic colitis in captivity [24]. This colitis is mainly related to the symptom-complex of the WMS, but also occurs independently. The clinical picture of diseased marmosets shows clear parallels with the Crohn's disease in humans. Due to the clinical and pathological similarities, cottontop tamarins were already used as an animal model for scientific studies on ulcerative colitis and Crohn's disease [25]. However, the frequently discussed etiological relationship between MAP and Crohn's disease [26], and the possible association with WMS, still remains unclear.

Moreover, it was striking that MAP DNA was detected in the bone marrow of the common marmoset (No.4). Only two other studies have previously reported the detection of MAP in the bone marrow. MAP was detected in the bone marrow of two stumptail macaques (Macaca arctoides) by microscopic examination [2] and in a German Fleckvieh bull (Bos primigenius taurus) by PCR [11]. In particular, these findings are of great interest, since recent studies have indicated that a MAP-related pathogen, Mycobacterium tuberculosis, persists within the CD271 ${ }^{+} / \mathrm{CD}^{-} 5^{-}$ mesenchymal stem cells of the bone marrow in a viable but non-culturable (VBNC) state, and seems to play a key role in the establishment of latent tuberculosis [27, 28]. Hence, bone marrow perhaps also provides a niche for latent infections with MAP in 
non-human primates. Furthermore, MAP cells in the VBNC state may explain in part the failure of MAP growth in this study, as MAP was only detectable by PCRs. Although it is known that MAP merge into a VBNC stage [29, 30], it is highly likely that cultivation failed due to small sample volumes as well as the low numbers of MAP cells, which were found typically in asymptomatically infected animals. Furthermore, also the HPC decontamination of the samples leads to a reduction of viability of the bacteria, especially in the case of rather small numbers of MAP [31]. The sensitivity of MAP cultivation from the feces of subclinically infected animals is considered to be 23$49 \%$, whereas cultivation of MAP from animals with clinical signs of paratuberculosis is thought to reach a sensitivity of $98 \%$ [32]. Also in other studies, a discrepancy between the results of PCR and cultivation were observed [9, 11, 14, 33]. In one of these previous studies, 875 of 1906 (45.91\%) investigated fecal samples from dairy cattle were tested positively for the presences of MAP DNA by an IS900 based realtime PCR. In contrast, growths of MAP colonies were only detected in 169 of 1906 $(8.87 \%)$ fecal samples, whereby all these samples were also tested positively by the real-time PCR [33].

The results of the present study confirmed MAP infections in two non-human primates kept in Germany due to the PCR positive results in the tissue samples. The incidence of MAP infections seems to be low and there is no direct correlation between the demonstration of the agent and the individual cause of disease or death. Therefore, an asymptomatic infection was assumed in the two positive cases. Nevertheless, by detecting MAP in the common marmoset, the number of non-ruminants infected with MAP was expanded. In particular, non-human primates are genetically and physiologically most similar to humans. Detailed analysis of MAP-infected non-human primates could promote further understanding of mechanisms in bowel diseases associated with a MAP infection or of MAP distribution in infected hosts. The redetection of MAP in the bone marrow highlighted that mesenchymal stem cells of the bone marrow could provide a niche for a latent paratuberculosis infection. Therefore, it is recommended that further studies include the examination of MAP in bone marrow, in addition to gastrointestinal tissues and lymph nodes. 


\section{Acknowledgements}

The authors wish to thank Julia Christine Steinfeldt and Wolfgang Henkel for their assistance in sample processing. We are grateful to Lynne Riddles for completing the critical review of the manuscript.

\section{References}

1 Beard PM, Rhind SM, Buxton D, Daniels MJ, Henderson D, Pirie A, Rudge K, Greig A, Hutchings MR, Stevenson K, Sharp JM. Natural paratuberculosis infection in rabbits in Scotland. J Comp Pathol 2001;124:290-299.

2 McClure HM, Chiodini RJ, Anderson DC, Swenson RB, Thayer WR, Coutu JA. Mycobacterium paratuberculosis infection in a colony of stumptail macaques (Macaca arctoides). J Infect Dis 1987;155:1011-1019.

3 Zwick LS, Walsh TF, Barbiers R, Collins MT, Kinsel MJ, Murnane RD. Paratuberculosis in a mandrill (Papio sphinx). J Vet Diagn Invest 2002;14:326328.

4 Stief B, Möbius P, Türk H, Hörügel U, Arnold C, Pöhle D. Paratuberculosis in a miniature donkey (Equus asinus f. asinus). Berl Münch Tierärztl Wochenschr 2012;125:38-44.

5 Whitlock RH, Buergelt C. Preclinical and clinical manifestations of paratuberculosis (including pathology). Vet Clin North Am Food Anim Pract 1996;12:345-356.

6 Singh SV, Singh AV, Singh PK, Kumar A, Singh B. Molecular identification and characterization of Mycobacterium avium subspecies paratuberculosis in free living non-human primate (Rhesus macaques) from North India. Comp Immunol Microbiol Infect Dis 2011;34:267-271.

7 Münster P, Fechner K, Völkel I, von Buchholz A, Czerny CP. Distribution of Mycobacterium avium ssp. paratuberculosis in a German zoological garden determined by IS900 semi-nested and quantitative real-time PCR. Vet Microbiol 2013;163:116-123. 
van Roermund HJ, Bakker D, Willemsen PT, de Jong MC. Horizontal transmission of Mycobacterium avium subsp. paratuberculosis in cattle in an experimental setting: calves can transmit the infection to other calves. Vet Microbiol 2007;122:270-279.

9 Kralik P, Pribylova-Dziedzinska R, Kralova A, Kovarcik K, Slana I. Evidence of passive faecal shedding of Mycobacterium avium subsp. paratuberculosis in a Limousin cattle herd. Vet J 2014;201:91-94.

10 Woo SR, Czuprynski CJ. Tactics of Mycobacterium avium subsp. paratuberculosis for intracellular survival in mononuclear phagocytes. J Vet Sci 2008;9:1-8.

11 Fechner K, Schäfer J, Wiegel C, Ludwig J, Münster P, Sharifi AR, Wemheuer W, Czerny CP. Distribution of Mycobacterium avium subsp. paratuberculosis in a Subclinical Naturally Infected German Fleckvieh Bull. Transbound Emerg Dis 2015:DOI 10.1111/tbed.12459.

12 Czerny CP, Münster P. Detection method for Mycobacterium avium ssp. paratuberculosis. Georg-August-University Göttingen Public Law Foundation. Patent WO 2013160434. October 31, 2013.

13 Li L, Bannantine JP, Zhang Q, Amonsin A, May BJ, Alt D, Banerji N, Kanjilal $\mathrm{S}$, Kapur V. The complete genome sequence of Mycobacterium avium subspecies paratuberculosis. Proc Natl Acad Sci U S A 2005;102:12344-12349.

14 Münster P, Völkel I, Wemheuer W, Schwarz D, Döring S, Czerny CP. A longitudinal study to characterize the distribution patterns of Mycobacterium avium ssp. paratuberculosis in semen, blood and faeces of a naturally infected bull by IS 900 semi-nested and quantitative real-time PCR. Transbound Emerg Dis 2013;60:175-187.

15 Schneider F. Entwicklung einer "seminested PCR" und einer "Real-TimePCR" zum Nachweis von Mycobacterium avium ssp. paratuberculosis bei Rindern (Development of a "semi-nested PCR" and a "real-time PCR" for the detection of Mycobacterium avium spp. paratuberculosis in cattle). $\mathrm{PhD}$ thesis, Ludwig-Maximilians-University, Munich, Bavaria, Germany 2003. 
16 Friedrich-Loeffler-Institut. Official Manual of Diagnostic Procedures. https://openagrar.bmel-

forschung.de/servlets/MCRFileNodeServlet/Document_derivate_00005327/TK1 8_Paratuberkulose_2014929.pdf;jsessionid=3308B9CA153B08F3548E5483D40 9A41C/. Friedrich- Loeffler-Institut, the German Federal Research Institute of Animal Health 2014.

17 Whittington RJ, Whittington AM, Waldron A, Begg DJ, de Silva K, Purdie AC, Plain KM. Development and validation of a liquid medium (M7H9C) for routine culture of Mycobacterium avium subsp. paratuberculosis to replace modified Bactec 12B medium. J Clin Microbiol 2013;51:3993-4000.

18 Carta T, Alvarez J, Perez de la Lastra JM, Gortazar C. Wildlife and paratuberculosis: a review. Res Vet Sci 2013;94:191-197.

19 Didier A, Mätz-Rensing K, Kuhn EM, Richter E, Kaup FJ. Brief communications and case reports - A case of intestinal Mycobacterium simiae infection in an SIV-infected immunosuppressed rhesus monkey. Vet Pathol 1999;36:249-252.

20 Castellanos E, Aranaz A, de Juan L, Alvarez J, Rodriguez S, Romero B, Bezos J, Stevenson K, Mateos A, Dominguez L. Single nucleotide polymorphisms in the IS900 sequence of Mycobacterium avium subsp. paratuberculosis are strain type specific. J Clin Microbiol 2009;47:2260-2264.

21 Semret M, Turenne CY, Behr MA. Insertion sequence IS900 revisited. J Clin Microbiol 2006;44:1081-1083.

22 Biet F, Sevilla IA, Cochard T, Lefrancois LH, Garrido JM, Heron I, Juste RA, McLuckie J, Thibault VC, Supply P, Collins DM, Behr MA, Stevenson K. Interand intra-subtype genotypic differences that differentiate Mycobacterium avium subspecies paratuberculosis strains. BMC Microbiol 2012;12:264.

23 Bannantine JP, Li L, Mwangi M, Cote R, Raygoza Garay JA, Kapur V. Complete Genome Sequence of Mycobacterium avium subsp. paratuberculosis, Isolated from Human Breast Milk. Genome Announc 2014;2.

24 Ludlage E, Mansfield K. Clinical care and diseases of the common marmoset (Callithrix jacchus). Comp Med 2003;53:369-382. 
25 Clapp NK, Henke MA, Hansard RM, Walsh RE, Widomski DL, Anglin CP, Fretland DJ, Gaginella TS. Inflammatory mediators in cotton-top tamarins (CTT) with acute and chronic colitis. Agents Actions 1991;34:178-180.

26 Atreya R, Bülte M, Gerlach GF, Goethe R, Hornef MW, Köhler H, Meens J, Möbius P, Roeb E, Weiss S, on behalf of the Zoo MAPC. Facts, myths and hypotheses on the zoonotic nature of Mycobacterium avium subspecies paratuberculosis. Int J Med Microbiol 2014;304:858-867.

27 Raghuvanshi S, Sharma P, Singh S, Van Kaer L, Das G. Mycobacterium tuberculosis evades host immunity by recruiting mesenchymal stem cells. Proc Natl Acad Sci U S A 2010;107:21653-21658.

28 Das B, Kashino SS, Pulu I, Kalita D, Swami V, Yeger H, Felsher DW, CamposNeto A. CD271(+) bone marrow mesenchymal stem cells may provide a niche for dormant Mycobacterium tuberculosis. Sci Transl Med 2013;5:170ra113.

29 Whittington RJ, Marshall DJ, Nicholls PJ, Marsh IB, Reddacliff LA. Survival and dormancy of Mycobacterium avium subsp. paratuberculosis in the environment. Appl Environ Microbiol 2004;70:2989-3004.

30 Lamont EA, Bannantine JP, Armien A, Ariyakumar DS, Sreevatsan S. Identification and characterization of a spore-like morphotype in chronically starved Mycobacterium avium subsp. paratuberculosis cultures. PLoS One 2012;7:e30648.

31 Bradner L, Robbe-Austerman S, Beitz DC, Stabel JR. Optimization of hexadecylpyridinium chloride decontamination for culture of Mycobacterium avium subsp. paratuberculosis from milk. J Clin Microbiol 2013;51:1575-1577.

32 Nielsen SS, Toft N. Ante mortem diagnosis of paratuberculosis: a review of accuracies of ELISA, interferon-gamma assay and faecal culture techniques. Vet Microbiol 2008;129:217-235.

33 Kralik P, Slana I, Kralova A, Babak V, Whitlock RH, Pavlik I. Development of a predictive model for detection of Mycobacterium avium subsp. paratuberculosis in faeces by quantitative real time PCR. Vet Microbiol 2011;149:133-138. 


\section{CHAPTER III}

Paper under review

Journal of Zoo and Wild Medicine

\section{Detection of Mycobacterium avium subsp. paratuberculosis in rock hyraxes} (Procavia capensis) imported from South Africa

Kim Fechner ${ }^{\mathrm{a}}$, Jenny Schäfer ${ }^{\mathrm{a}}$, Pia Münster ${ }^{\mathrm{a}}$, Kerstin Ternes $^{\mathrm{b}}$, Susanne Döring ${ }^{\mathrm{a} \ddagger}$, Inger Völkel $^{\mathrm{a} \dagger}$, Franz-Josef Kaup ${ }^{\mathrm{c}}$ and Claus-Peter Czerny ${ }^{\mathrm{a}^{*}}$

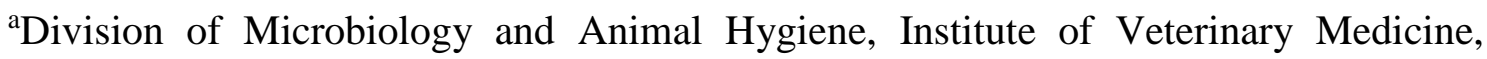
Department of Animal Sciences, Faculty of Agricultural Sciences, Georg-AugustUniversity Göttingen, Burckhardtweg 2, D-37077 Göttingen, Germany.

bZoo Duisburg, Mülheimer Straße 273, D-47058 Duisburg, Germany.

'Division of Infectious Pathology, German Primate Center (DPZ), Kellnerweg 4, D37077 Göttingen, Germany.

\#Present address. H. Bröring GmbH \& Co. KG, Ladestr. 2, D-49413 Dinklage, Germany.

Fresent address. Department of Agriculture, South Westphalia University of Applied Sciences, Lübecker Ring 2, North Rhine-Westphalia, D-59494 Soest, Germany.

†Present address. National Veterinary Research Office Arnsberg, Zur Taubeneiche 10 12, D-59821 Arnsberg, Germany.

*Corresponding author. Mailing address: Department of Animal Sciences, GeorgAugust-University, Burckhardtweg 2, D-37077 Göttingen, Germany.

Phone: +49 55139 33375; Fax: +49 55139 13513; E-mail address: cczerny@ gwdg.de. 


\begin{abstract}
Mycobacterium avium subspecies paratuberculosis (MAP) causes chronic, progressive and consecutively fatal enteritis, especially in ruminants. MAP distribution among wildlife is not yet clear. In this study, three wild-born rock hyraxes (Procavia capensis) had been imported from South Africa to a German zoological garden. During the quarantine period, four young animals were born. The wild-born animals showed symptoms of mild diarrhea shortly after their arrival in the zoological garden, but all routine parasitological and bacteriological tests performed were negative. Therefore, the animals were additionally tested for MAP infection. MAP DNA was detected by seminested PCR (snPCR) in a pooled fecal sample of the seven animals. Subsequent PCR analysis of the individual feces samples confirmed the excretion of MAP in two rock hyraxes (one wild-born and one born in captivity). Sequence analysis of the corresponding 278 bp amplicons revealed 100\% homology to the reference MAP-K10 IS900 sequence. No antibody response against MAP was detected in the individual serum samples. MAP-specific post-mortem lesions were not observed by gross pathology and histology, neither after death nor after euthanization of the animals. Nevertheless, MAP was detected by snPCR and culture in the gastrointestinal tract, urogenital tract, cardiovascular system and/or respiratory system of three other animals of the group (one wild-born and two born in captivity). This study is the first report confirming MAP occurrence in rock hyraxes. Therefore, it is recommended that especially veterinarians and zoo employees consider rock hyraxes as a possible source of MAP infection for domestic livestock in South Africa and the valuable animal stock of zoological facilities.
\end{abstract}

Key words: bacterial culture, Mycobacterium avium subsp. paratuberculosis, PCR, Procavia capensis, rock hyraxes, wildlife, zoological garden 


\section{Introduction}

The rock hyrax (Procavia capensis) - also known as dassie rat - is one of the four species of the order Hyracoidea, which belong to the clade of Paenungulata together with elephants (Elephantidae) and sea cows (Sirenia). Rock hyraxes represent a species with a weight of 2.5 to $4 \mathrm{~kg}$, widely found in South Africa and the Middle East. Due to the extermination of predators such as black-backed jackal and lynx during the early 1960s, the number of rock hyraxes increased exponentially. In some areas of South Africa, they were officially listed as vermin. ${ }^{15}, 17$ Currently, the population trend is stable, ${ }^{2}$ although it is regularly reported that rock hyrax invasions exist in residential areas. $^{38}$ Moreover, rock hyraxes pose an agricultural problem as they compete with domestic livestock for available grazing space. ${ }^{15,17}$

First reports on mycobacterial infections of rock hyraxes were published more than fifty years ago, ${ }^{35,} 36$ when the so-called dassie bacillus was detected. Since then, the dassie bacillus has repeatedly been isolated from rock hyraxes in South Africa, ${ }^{30,} 35$ as well as in zoo facilities in Australia and Canada. ${ }^{4,} 19$ The dassie bacillus was formerly classified as an acid-fast bacterium resembling Mycobacterium microti, the causative agent of tuberculosis in rodents. ${ }^{32}$ However, recent genomic analyzes have demonstrated that the dassie bacillus is more closely related to chimpanzee bacillus, Mycobacterium mungi and/or Mycobacterium suricattae. ${ }^{6}$ Mycobacterium africanum was also reported to cause infection in rock hyraxes, ${ }^{12}$ but it should be mention that the used method is not able to differentiate between Mycobacterium africanum and the dassie bacillus. Therefore, it is possible that identified bacteria might also belong to the dassie bacillus. However, the detection of Mycobacterium avium subspecies paratuberculosis (MAP) was never described in rock hyraxes.

MAP is the infectious agent of paratuberculosis, which mainly affects small and large domestic ruminants. In ruminants the disease is characterized by a degenerative, chronic granulomatous inflammation of the intestinal tract, which is often accompanied by diarrhea, weight loss, reduced reproductive performance and eventually death. ${ }^{13}$ In addition to domestic ruminants, MAP has also been reported to infect a broad range of other domesticated, wild, and zoo animals including non-ruminants. ${ }^{1,11,23}$ 
This study provides a detailed analysis of the presence of MAP in a group of rock hyraxes, which were once captured in South Africa and kept in quarantine at a German zoological garden.

\section{Materials and methods}

\section{Animal samples}

Three wild-born rock hyraxes (Procavia capensis; animals 3, 4, 5) were imported from South Africa to the Zoo Duisburg $\left(51^{\circ} 26^{\prime} 12^{\prime \prime} \mathrm{N}, 6^{\circ} 48^{\prime} 14^{\prime \prime} \mathrm{E}\right)$ in North RhineWestphalia, Germany (Table 1). During the course of the examination, four rock hyraxes (animals 1, 2, 6, 7) were born in captivity. The animals were not released from quarantine during the entire investigation period from 2007 until 2009. The wild-born hyraxes showed repeatedly mild symptoms of diarrhea that started five days after their arrival at the zoological garden. Repeated routine parasitological and bacteriological examinations of their feces excluded pathogens and parasites causing diarrhea, such as Salmonella spp., Escherichia coli, Shigella spp., Campylobacter spp., Clostridium spp., or Cryptosporidia. Therefore, an infection with MAP was considered. To prove this suspicion, a pooled fecal sample of all seven rock hyraxes was tested for MAP DNA by semi-nested PCR with a positive result. Afterwards, individual fecal and blood samples of the animals were collected. During the period from May 2008 to July 2009, three rock hyraxes $(1,2,7)$ died and the other four animals $(3,4,5,6)$ were euthanized in the zoological garden as a result of their presumed infection status. Animal 7 died as a result of an acute cardiovascular failure during anesthesia. Animal 1 had acute diarrhea and died suddenly without significant findings. The unexpected death of animal 2 could not be explained because no clinical symptoms were observed. Although the carcasses were put immediately on ice and delivered within $24 \mathrm{~h}$ for post-mortem examinations to the Institute of Veterinary Medicine in Göttingen, tissue samples were observed to be moderately decayed in animals 1, 4 and 5, whereas animals 2, 3, 6 and 7 showed a higher degree of decay. This may be due to the dense coat of the rock hyraxes inhibiting to give off body heat. During necropsy, gross pathological changes were documented and different tissue samples from the gastrointestinal tract and appending organs, the cardiovascular system, respiratory system and urogenital tract were collected under 


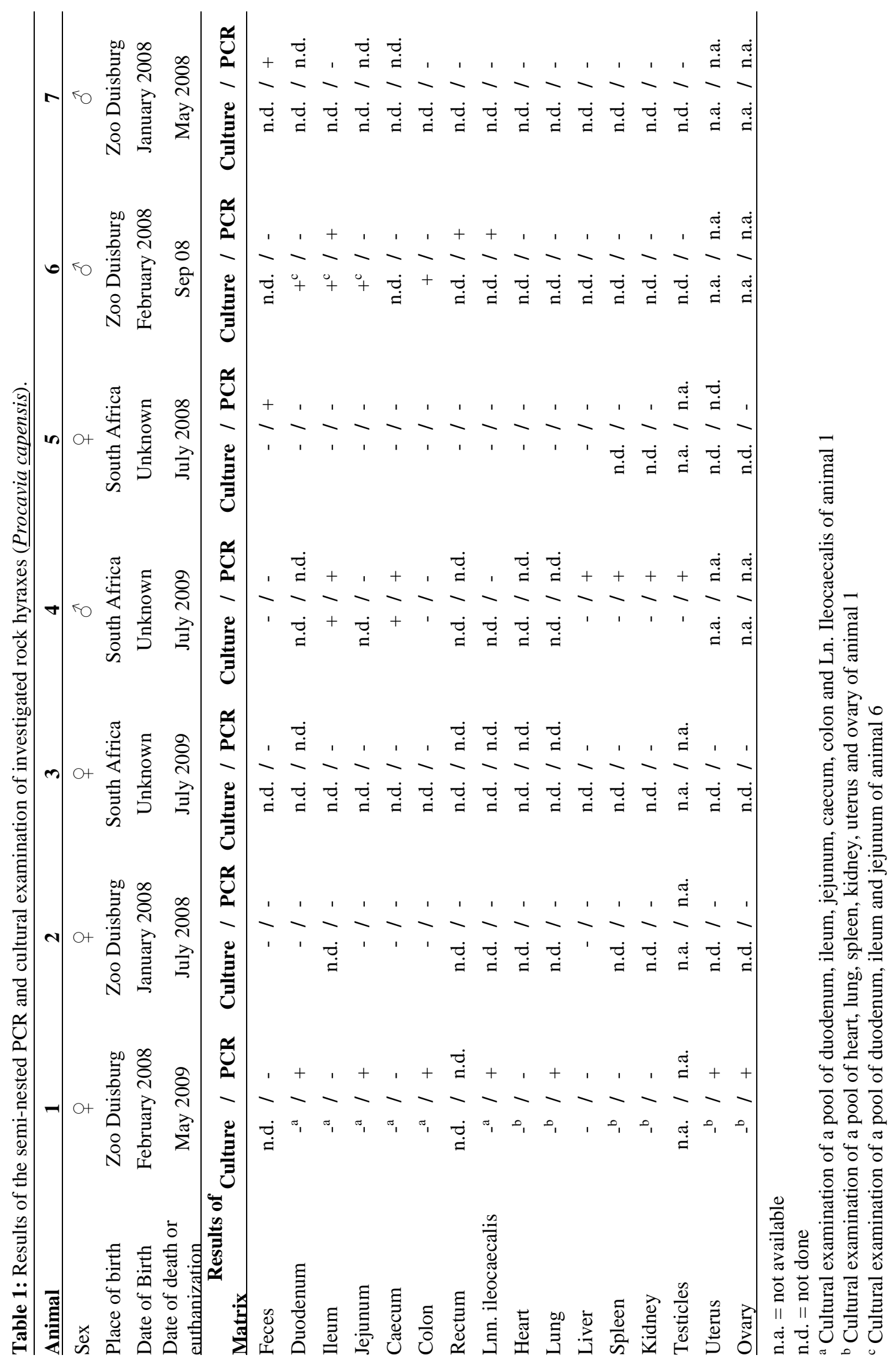


sterile conditions (Table 1). Due to autolysis, the tissue analyses were not performed uniformly for all animals (Table 1). Furthermore, samples of the gastrointestinal tract were transferred into a $10 \%$ formaldehyde solution for histopathological investigations. To avoid cross-contamination, new sterile surgical gloves, plastic forceps and scalpels were used for each tissue sample.

\section{DNA extraction}

DNA was extracted and purified from fecal samples in duplicates using a modified protocol of the QIAamp Blood Kit (Qiagen, D-40724 Hilden, North Rhine-Westphalia, Germany). Briefly, $50 \mathrm{mg}$ feces were transferred into sterile $2 \mathrm{ml}$ tubes, mixed with 100 $\mu \mathrm{l}$ PBS and ultra-sonicated thrice for $10 \mathrm{sec}$ at $20 \mathrm{kHz}$ (Bendelin Sonoplus HD 2200, BANDELIN electronic GmbH \& Co. KG, D-12207 Berlin, Germany). Subsequently, $360 \mu \mathrm{l}$ lysis buffer (AL-Buffer) was added, and the samples were incubated on a thermomixer at $37^{\circ} \mathrm{C}$ for $30 \mathrm{~min}$ with continuous mixing at $900 \mathrm{rpm}$. Afterwards, $60 \mu \mathrm{l}$ proteinase $\mathrm{K}$ solution $(20 \mathrm{mg} / \mathrm{ml})$ and $600 \mu \mathrm{l}$ lysis buffer were added. The tubes were then incubated on the thermomixer at $56^{\circ} \mathrm{C}$ with shaking at $900 \mathrm{rpm}$ for $30 \mathrm{~min}$, followed by further incubation for $15 \mathrm{~min}$ at $95^{\circ} \mathrm{C}$ and $900 \mathrm{rpm}$.

After applying the lysis steps, the following DNA precipitation with ethanol ( $\geq 99.8 \%)$, loading of the QIAamp Spin Column and two-step washing was performed according to the corresponding kit manual. Finally, DNA was eluted in $200 \mu 1$ elution buffer (AEbuffer) at $85^{\circ} \mathrm{C}$ and stored at $-20^{\circ} \mathrm{C}$ until further use.

To extract the DNA from the tissue samples $(50 \mathrm{mg})$ in duplicate, the QIAamp DNA Mini Kit (Qiagen, D-40724, Hilden, North Rhine-Westphalia, Germany) was used according to kit protocol.

\section{Qualitative semi-nested polymerase chain reaction}

A qualitative semi-nested PCR (snPCR) based on the insertion sequence "IS900" was performed as described previously. ${ }^{23,}{ }^{24}$ The primers MAPfor1 (5'-GTC GGC GTG GTC GTC TGC TGG GTT GAT-‘3) and MAPrev (5'-GCG CGG CAC GGC TCT TGT TGT AGT C-‘3) amplified a 587 bp fragment in the first run, while the MAPrev primer - together with the forward primer MAPfor2 (5'- CGG GCG CAC GGT CCA TCA CG'3) - produced a $278 \mathrm{bp}$ fragment in the second run. As a positive control, DNA was extracted from a MAP reference strain ordered from the German Collection of 
Microorganisms and Cell Cultures (DSMZ, D-38124, Braunschweig, Lower Saxony, Germany; ID: 44133). Sterile distilled water was used as a negative control.

\section{Cloning and sequencing}

To confirm MAP specificity, the 278 bp fragments obtained from snPCR of the fecal samples - covering nucleotide position 492 to 770 within the IS900 (GenBank: AE016958) - were inserted into the $\mathrm{pCR}^{\circledR} 2.1-\mathrm{TOPO}^{\circledR}$ plasmid vector (Fisher Scientific GmbH, D-58239 Schwerte, North Rhine-Westphalia, Germany). Afterwards, the plasmid vector was transformed into $E$. coli according to the manufacturer's instructions (Wizard $^{\circledR}$ Plus SV Minipreps Purification System, Promega, D-68199, Mannheim, Baden-Wuerttemberg, Germany). Plasmid DNA was isolated from two clones per amplicon using QIAprep ${ }^{\circledR}$ Spin Miniprep Kit (Qiagen, D-40724 Hilden, North RhineWestphalia, Germany). The inserts were sequenced in both strand directions using the M13 primers (The Goettingen Genomics Laboratory, D-37077 Goettingen, Lower Saxony, Germany). The obtained sequences were compared to all sequences from GenBank entries (www.ncbi.nlm.nih.gov) by BLAST search. To monitor the degree of similarity, an alignment with published IS900 sequences was performed using "MegAlign" (DNAStar, Inc., Madison, Wisconsin 53705, USA).

\section{Cultural examination of MAP}

MAP growth from fecal and tissue samples were carried out on Herrold's egg yolk medium (HEYM) obtained from Becton, Dickinson and Company (D-69126 Heidelberg, Baden-Wuerttemberg, Germany). The procedure was performed as described in the German reference protocol. ${ }^{9,}{ }^{14}$ In addition, feces spiked with a MAP reference strain (DSMZ ID: 44133) were used as a positive control. Growth of bacterial cultures was monitored for the first time after four weeks, and then regularly every four weeks for up to 16 weeks. Cultures exhibiting suspicious colonies were verified by the snPCR as mentioned above.

\section{Enzyme-linked immunosorbent assay}

To analyze the antibody response to MAP, a slightly modified procedure from a protocol published previously using the HerdChek M.pt Ab (IDEXX GmbH, D-71636 Ludwigsburg, Baden-Wuerttemberg, Germany) was applied. ${ }^{34}$ Twenty microliters of the rock hyrax serum samples were mixed with $180 \mu \mathrm{l}$ absorbent solution and incubated at RT for $60 \mathrm{~min}$. The pre-incubated samples were transferred to the MAP antigen-coated 
96-well microplate and incubated at RT for $60 \mathrm{~min}$. The plate was washed five times with the wash solution and incubated for $60 \mathrm{~min}$ at RT with $100 \mu \mathrm{l}$ of the absorbent solution. All wells were washed five times. Next, $100 \mu 1$ horseradish peroxidase (HRP) protein-G conjugate, reacting with antibodies of multiple animal species, was added to each well and incubated for further $30 \mathrm{~min}$ at RT. The wells were washed five times again and $100 \mu \mathrm{l}$ of the substrate solution was added. Directly, the plate was incubated in a closed dark chamber for $10 \mathrm{~min}$ at $\mathrm{RT}$, before $100 \mu \mathrm{l}$ of the stop solution were added. The optical density (OD) was measured at $450 \mathrm{~nm}$ wavelength to calculate the $\mathrm{S} / \mathrm{P}$ ratios. The cut-offs were applied as recommended by the manufacturer for ruminants: serum samples $\leq 0.15 \mathrm{~S} / \mathrm{P}$ negative $(-),>0.15-<0.3 \mathrm{~S} / \mathrm{P}$ borderline $( \pm), \geq 0.3$ S/P positive (+). The positive and negative controls supplied by the HerdChek M.pt Ab ELISA kit were used as described by the manufacturer.

\section{Histopathology and immunohistochemistry}

For histopathological examination, tissue samples were fixed in $10 \%$ neutral buffered formalin and embedded in paraffin. The prepared sections were stained with standard eosin-hematoxylin (HE) using the Varistain Gemini staining device (Thermo Fisher, Scientific, D-64293 Darmstadt, Hesse, Germany). Sections of small and large intestines were stained with Ziehl-Neelsen using standardized protocols to detect acid-fast bacteria. In addition, immunohistochemistry was performed as described elsewhere using a rabbit polyclonal antibody against Mycobacterium bovis (DAKO, D-22083 Hamburg, Germany). ${ }^{5}$

\section{Results}

Physical condition, sensorium and eating habits of each animal were good and the fecal consistencies were normal most of the time, except for episodes of mild diarrhea. Due to the clinical symptomatic, the exclusion of several other diarrhea pathogens and the fact that rock hyraxes are susceptible to different mycobacteria, it was presumed that these group of rock hyraxes may be infected with MAP. In a pooled fecal sample from all colony members the presence of MAP DNA was confirmed by snPCR (Figure 1A). Afterwards, analysis of individual fecal, blood and tissue samples verified the occurrence of MAP in the rock hyraxes. 
Detection of MAP DNA in individual fecal and tissue samples by snPCR and sequencing of the PCR amplicons

Analysis of individual samples revealed MAP DNA in the feces of animals 5 and 7, but not in their internal organs (Figure 1B, Table 1). Sequences of the DNA (278 bp) amplified from both samples were aligned to the MAP-K10 IS900 reference sequence (GenBank: AE016958). A homology degree of 100\% was recorded. Interestingly, MAP DNA was detected in the tissue samples mainly in the gastrointestinal tract, but also in heart, lung, liver, spleen, kidney, and reproduction organs of three other rock hyraxes (1, 4 and 6) with negative results in the corresponding fecal samples (Table 1). Both fecal and tissue samples of the residual two animals ( 2 and 3 ), were tested negative for MAP in the snPCR.

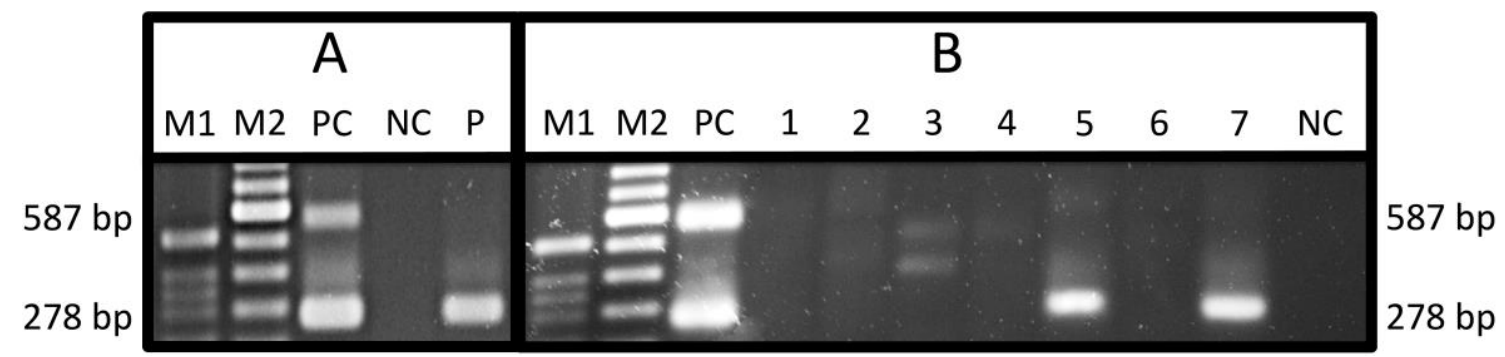

Figure 1: Detection of MAP DNA in the pooled fecal sample (A) and individual fecal samples (B) collected from a group of seven rock hyraxes (Procavia capensis) by an IS900 based semi-nested PCR, visualized by gel electrophoresis. Depending on the MAP DNA concentration, either the two specific 587 bp and 278 bp fragments or the $278 \mathrm{bp}$ fragment alone were amplified and demonstrate a positive finding. M1, $1 \mathrm{~kb}$ molecular weight marker; M2, 100 bp molecular weight marker; PC, positive control; lane $\mathrm{P}$, the pooled fecal sample; lanes 1 to 7 ; individual fecal samples of rock hyrax 1 to 7; NC, negative control.

\section{Cultural examination of MAP in individual fecal and tissue samples}

Fecal and tissue samples were prepared for cultivation of MAP on HEYM. Unfortunately, due to the high degree of decay in the animals, no tissue samples from animals 3 and 7, as well as some single organ samples from the other animals, were prepared for the cultural examination (Table 1). MAP colonies were detected and 
confirmed by snPCR in the tissue samples of two out of five investigated animals. Growth of MAP colonies was detected in the ileum and caecum tissue samples of animal 4, an imported animal. Furthermore, MAP was isolated from a pool of small intestine section samples and the colon of animal 6. MAP was not cultivable from samples of animals 1, 2 and 5 .

\section{Serological investigations}

Serum samples of all rock hyraxes were analyzed by a commercially available ELISA (HerdChek M.pt Ab; IDEXX). Positive and negative controls confirmed the validity of the performed ELISA as specified by the manufacturer. The S/P ratio of the rock hyrax sera was below 0.15 , and therefore, no antibody response was measured.

\section{Gross pathological, histopathological and immunohistochemical findings}

Five rock hyraxes (1, 3, 4, 5 and 7) were examined histopathologically. Although no typical, gross morphological changes, which may be related to a MAP infection, were observed, the histological examinations showed inflammatory cell infiltrates of the intestinal wall. In detail, the lamina propria and submucosa of the intestines of animal 5 contained numerous inflammatory cells including lymphocytes, neutrophilic and eosinophilic granulocytes. Some of the macrophages carried intracytoplasmatic eosinophilic structures, but MAP was not detected within these cells. Various submucosal locations showed secondary lymphoid tissue. Mesenteric lymph nodes were characterized by a moderate hyperplasia accompanied by accumulations of macrophages and sporadic giant cells. These results could be a sign of indicating minor subacute enteritis. Animal 1 had diarrhea and died suddenly. The intestinal mucosal presented extensive destruction, hindering adequate histologic analysis. Nevertheless, some intestinal sections showed moderate GALT hyperplasia and local presence of multiple small mineralized foci. Animals 3 and 4 showed similar alterations of the gut, the small and the large intestine were characterized by multifocal occurrence of lymphoplasmacytic inflammation and an increased presence of eosinophil granulocytes in the lamina propria. In the lamina propria and submucosa of the small intestine, multifocally lymphocytes, macrophages and polymorphonuclear neutrophils and eosinophils were observed. The small and large intestine of animal 7 were without any significant findings. Acid-fast bacteria could not be detected in the tissue of any animal by Ziehl-Neelsen staining. The results of the immunohistochemical examinations of the 
intestinal tract were invalid, due to a large number of autolysis-induced nonspecific findings.

\section{Discussion}

Studies on infections of rock hyraxes with mycobacteria other than MAP have already been published. $4,12,19,30,35,36$ Nevertheless, this is the first report on the isolation of MAP in these animal species. MAP was detected in the gastrointestinal tract, urogenital tract, cardiovascular system and/or respiratory system of three rock hyraxes (one wildborn and two born in captivity) and in the feces of two others (one wild-born and one born in captivity) by snPCR and/or culture. The 278 bp amplicons of the detected DNA in the fecal samples were identical to the IS900 reference sequence of the MAP-K10 Genome (GenBank: AE016958), confirming the identity of MAP.

Although in most rock hyraxes (5 animals) MAP was detected, both gross pathological and pathohistological findings did not show any distinctive signs of paratuberculosis. However, these investigations were impeded by the autolysis of the carcasses whereby the validity of the results might be negatively influenced. The only clinical finding, which may be related to an infection with MAP, was the mild, irregular diarrhea of the investigated animals. These findings are similar to the early phase or subclinical infections described in cattle. ${ }^{8,25,37}$ Symptoms are mild and restricted to irregular, short periods of soft feces accompanied by the excretion of low amounts of MAP. While paratuberculosis is well documented for domestic cattle, little is known about pathogenesis and course of the infection in non-ruminants. In previous studies on nonruminant species such as foxes (Vulpes vulpes), stoats (Mustela erminea), weasels (Mustela nivalis), crows (Corvus corone), and wood mice (Apodemus sylvaticus) infected with MAP were reported. However, in these animals mostly no or just mild gross pathological changes and histopathological lesions were found. ${ }^{1,3,11}$ Therefore, it could be assumed that MAP may replicate in rock hyraxes without triggering characteristic macroscopic or microscopic lesions. Up to now, the only non-ruminant species, which is known to develop typical histopathological changes for paratuberculosis, is the rabbit (Oryctolagus cuniculus). ${ }^{3}$

It is assumed that paratuberculosis is present in most countries. ${ }^{26}$ Especially in South Africa, the disease seems to be an emerging problem in sheep. ${ }^{20}$ Therefore, it is most 
likely that the wild-born rock hyraxes were naturally infected in South Africa and were responsible for the infection of the young animals, which were born in captivity. Improbable, but not completely excludable is an infection of the wild-born rock hyraxes with MAP whilst being transported from South Africa to Germany and/or during the quarantine period, although strict hygiene interventions were met. However, only very little information are available on the occurrence and host range of MAP in Africa. Sheep, ${ }^{20,29}$ goats, ${ }^{20,22,27}$ camels, ${ }^{27}$ as well as exotic and local breeds of cattle are known to be domesticated hosts of MAP in Africa. ${ }^{21,28}$ To our knowledge, the only record of paratuberculosis in the African wildlife is the serological detection of MAP antibodies in antelopes (20/373) from Zambia. ${ }^{16}$

In other regions of the world, it is known that besides several ruminant wildlife species, non-ruminant species are also reservoirs for MAP, such as wild rabbits in Scotland. ${ }^{1,3,11}$ Especially in East Scotland, the high prevalence of MAP in rabbits is associated with difficulties in controlling paratuberculosis on cattle farms. ${ }^{31}$ Therefore, rock hyraxes may play a role in the indirect transmission of MAP to domestic ruminants in South Africa since they share the same grasslands. ${ }^{15,17}$ For epidemiological tracing of species transmission between wild and domestic animals or different livestock herds, multitarget genotyping seems to be a suitable tool. ${ }^{10,33}$ By the combination of different tools like short sequence repeat (SSR) analysis, mycobacterial interspersed repetitiveunit-variable-number tandem-repeat (MIRU-VNTR) analysis, and restriction fragment length polymorphism analysis based on IS900 (IS900-RFLP) a transmission of MAP between wild-living red deer (Cervus elaphus) and farmed cattle herds, which shared the same habitats, was demonstrated previously. ${ }^{10}$ Therefore, this should further be investigated in rock hyraxes sharing grassland with domesticated ruminants in Africa.

In addition to the potential risk of transmission to the domestic livestock, previous research data shows that captured wild animals may be a source of the infection for the valuable animal stock of zoo facilities. ${ }^{23,34}$ In particular, this relates to the introduction of new zoo animals infected subclinically with MAP. The long incubation period without visible clinical signs result in a significant unrecognized spread of the bacteria to a former paratuberculosis-free collection. While MAP-infected domestic ruminants were usually culled, ${ }^{18}$ this method is not suitable for zoo animals, especially for endangered species. Therefore, a recommended focus is on the prevention of the infection by enforcing strict hygiene standards during the quarantine period. In addition, 
standard hygiene measures such as restricting access to the animal enclosure for a few authorized staff members, and using protective clothing and utensils must be applied.

An essential requirement for preventing and controlling paratuberculosis is the rapid identification of asymptomatic MAP shedders. This strategy is necessary for animal stocks of zoo facilities as well as for livestock farms. Likewise, the introduction of MAP infected animals from exogenous sources was prevented by the verification of the MAP infection status during the quarantine period. For such a survey, the analysis of pooled fecal samples seems to be an ideal approach, since this is an economical, as well as a rapid procedure for identifying MAP excretion. Of course, the implementation of individual sampling is not always possible in the daily zoo and farm routine due to time and economic reasons.

In one previous study, antibodies against MAP were detected successfully in eleven different zoo animal species by the same ELISA as used in the present study, including one non-ruminant species, the Malayan tapir (Tapirus indicus).$^{34}$ This indirect ELISA assay based on a protein $G$ conjugate, which is known to reacts with antibodies of multiple animal species and seems to be a useful alternative to species-specific secondary antibodies, especially for the examination of exotic animal species. In contrast, in our investigations, no antibody response against MAP was measurable. Generally, the presence of an antibody response during the early phase of the MAP infection is infrequent. ${ }^{7}$ Furthermore, it is known that ruminants could develop no antibody response at all or an inconsistent antibody response against MAP. ${ }^{8}{ }^{25}$ Currently, neither antigenic nor serological detection methods are the most favourable option for the diagnosis of paratuberculosis during the subclinical stage of the disease. Therefore, combinations of both methods, as well as several repetitions at different time points at individual level, are necessary for a reliable diagnosis of paratuberculosis.

\section{Conclusion}

To our knowledge, the present study is the first report on the detection of MAP in rock hyraxes. The results indicate that MAP is a pathogen of free-living rock hyraxes, which represents a potential source of infection for the valuable animal stock of zoo facilities and may play a role as a natural MAP-reservoir in South Africa. Therefore, it is our recommendation that especially veterinarians and zoo employees should consider the 
possibility of a MAP infection in animals captured in the wild. It may also be advisable to evaluate the MAP infection status of rock hyraxes in order to control the spread of MAP.

\section{Acknowledgments}

This work was funded by the Federal Ministry of Economics and Technology under support code KF2484201AJ9. The authors wish to thank Simone Urstadt for her excellent assistance in sample processing. We are grateful to Lynne Riddles and Ahmed Abd El Wahed for completing the critical review of this manuscript.

\section{Literature cited}

1. Beard PM, Daniels MJ, Henderson D, Pirie A, Rudge K, Buxton D, Rhind S, Greig A, Hutchings MR, McKendrick I, Stevenson K, Sharp JM. Paratuberculosis infection of nonruminant wildlife in Scotland. J Clin Microbiol 2001; 39: 1517-1521.

2. Butynski T, Hoeck H, Koren L, de Jong YA. Procavia capensis. The IUCN Red List of Threatened Species 2015: e.T41766A21285876. 2015; http://dx.doi.org/10.2305/IUCN.UK.2015-2.RLTS.T41766A21285876.en. : Downloaded on 26 February 2016.

3. Carta T, Alvarez J, Perez de la Lastra JM, Gortazar C. Wildlife and paratuberculosis: a review. Res Vet Sci 2013; 94: 191-197.

4. Cousins DV, Peet RL, Gaynor WT, Williams SN, Gow BL. Tuberculosis in imported hyrax (Procavia capensis) caused by an unusual variant belonging to the Mycobacterium tuberculosis complex. Vet Microbiol 1994; 42: 135-145.

5. Didier A, Mätz-Rensing K, Kuhn EM, Richter E, Kaup FJ. Brief communications and case reports - A case of intestinal Mycobacterium simiae infection in an SIV-infected immunosuppressed rhesus monkey. Vet Pathol 1999; 36: 249-252. 
6. Dippenaar A, Parsons SD, Sampson SL, van der Merwe RG, Drewe JA, Abdallah AM, Siame KK, Gey van Pittius NC, van Helden PD, Pain A, Warren RM. Whole genome sequence analysis of Mycobacterium suricattae. Tuberculosis (Edinb) 2015; 95: 682-688.

7. Dudemaine PL, Fecteau G, Lessard M, Labrecque O, Roy JP, Bissonnette N. Increased blood-circulating interferon-gamma, interleukin-17, and osteopontin levels in bovine paratuberculosis. J Dairy Sci 2014; 97: 3382-3393.

8. Fechner K, Schäfer J, Wiegel C, Ludwig J, Münster P, Sharifi AR, Wemheuer W, Czerny CP. Distribution of Mycobacterium avium subsp. paratuberculosis in a Subclinical Naturally Infected German Fleckvieh Bull. Transbound Emerg Dis 2015: DOI 10.1111/tbed.12459.

9. Friedrich-Loeffler-Institut. Amtliche Methodensammlung Paratuberkulose. [Official Manual of Diagnostic Procedures for Paratuberculosis] [Internet]. Friedrich-Loeffler-Institut, the German Federal Research Institute of Animal Health; [cited 2014 Marc 31]. Available from https://openagrar.bmelforschung.de/servlets/MCRFileNodeServlet/Document_derivate_00005327/TK1 8_Paratuberkulose_2014929.pdf;jsessionid=3308B9CA153B08F3548E5483D40 $9 \mathrm{~A} 41 \mathrm{C} /$

10. Fritsch I, Luyven G, Köhler H, Lutz W, Möbius P. Suspicion of Mycobacterium avium subsp. paratuberculosis transmission between cattle and wild-living red deer (Cervus elaphus) by multitarget genotyping. Appl Environ Microbiol 2012; 78: 1132-1139.

11. Greig A, Stevenson K, Henderson D, Perez V, Hughes V, Pavlik I, Hines ME, 2nd, McKendrick I, Sharp JM. Epidemiological study of paratuberculosis in wild rabbits in Scotland. J Clin Microbiol 1999; 37: 1746-1751.

12. Gudan A, Artukovic B, Cvetnic Z, Spicic S, Beck A, Hohsteter M, Naglic T, Bata I, Grabarevic Z. Disseminated tuberculosis in hyrax (Procavia capensis) caused by Mycobacterium africanum. J Zoo Wildl Med 2008; 39: 386-391.

13. Harris NB, Barletta RG. Mycobacterium avium subsp. paratuberculosis in Veterinary Medicine. Clin Microbiol Rev 2001; 14: 489-512. 
14. Köhler H, Soschinka A, Meyer M, Kather A, Reinhold P, Liebler-Tenorio E. Characterization of a caprine model for the subclinical initial phase of Mycobacterium avium subsp. paratuberculosis infection. BMC Vet Res 2015; 11: 74 .

15. Kolbe FF. A plea for dassies. Afr Wildl 1967; 21: 237-248.

16. Krauss H, Roettcher D, Weiss R, Danner K, Hübschle OJB. Wild animals as source of infection for domestic animals: investigations in Zambia. Giessener Beiträge zur Entwicklungsforschung: Reihe 1 1984; 10: 133-149.

17. Lensing JE. Intensity distribution patterns for five species of problem animals in south west africa. MADOQUA 1976; 10: 131-141.

18. Lu Z, Mitchell RM, Smith RL, Van Kessel JS, Chapagain PP, Schukken YH, Grohn YT. The importance of culling in Johne's disease control. J Theor Biol 2008; 254: 135-146.

19. Lutze-Wallace C, Turcotte C, Glover G, Cousins D, Bell J, Berlie-Surujballi G, Barbeau Y, Randall G. Isolation of a Mycobacterium microti-like organism from a rock hyrax (Procavia capensis) in a Canadian zoo. Can Vet J 2006; 47: 10111013.

20. Michel AL, Bastianello SS. Paratuberculosis in sheep: an emerging disease in South Africa. Vet Microbiol 2000; 77: 299-307.

21. Mohammed KB, El-Eragi AMS, Zakia AM. Seroprevalence of Bovine Paratuberculosis Specific Antibodies in Khartoum and Al-Jazeera States, Sudan. J Anim Vet Adv 2010; 9: 2098-2101.

22. Mpenda F, Buza J. Seroprevalence of Paratuberculosis in Goats and Sheep in Arusha, Northern Tanzania. Int J Sci Res 2014; 3: 541-545.

23. Münster P, Fechner K, Völkel I, von Buchholz A, Czerny CP. Distribution of Mycobacterium avium ssp. paratuberculosis in a German zoological garden determined by IS900 semi-nested and quantitative real-time PCR. Vet Microbiol 2013; 163: 116-123.

24. Münster P, Völkel I, Wemheuer W, Petschenka J, Wemheuer W, Steinbrunn C, Campe A, Schulz-Schaeffer WJ, Kreienbrock L, Czerny CP. Detection of 
Mycobacterium avium ssp. paratuberculosis in ileocaecal lymph nodes collected from elderly slaughter cows using a semi-nested IS900 polymerase chain reaction. Vet Microbiol 2011; 154: 197-201.

25. Münster P, Völkel I, Wemheuer W, Schwarz D, Döring S, Czerny CP. A longitudinal study to characterize the distribution patterns of Mycobacterium avium ssp. paratuberculosis in semen, blood and faeces of a naturally infected bull by IS 900 semi-nested and quantitative real-time PCR. Transbound Emerg Dis 2013; 60: 175-187.

26. Okuni JB. Occurrence of Paratuberculosis in African Countries: a Review. . J Vet Adv 2013; 3: 1-8.

27. Paling RW, Waghela S, Macowan KJ, Heath BR. The occurrence of infectious diseases in mixed farming of domesticated wild herbivores and livestock in Kenya. II. Bacterial diseases. J Wildl Dis 1988; 24: 308-316.

28. Pandey GS, Musonda TL, Chizyuka HG, Schneebeli M. Paratuberculosis (Johne's disease) in a herd of Friesian cattle in Zambia. Vet Rec 1987; 120: 369.

29. Pandey GS, Shimizu K, Orino K, Schneebeli M. Preliminary observations on ovine paratuberculosis (Johne's disease) in Zambia. Rev Elev Med Vet Pays Trop 1990; 42: 515-516.

30. Parsons S, Smith SGD, Martins Q, Horsnell WGC, Gous TA, Streicher EM, Warren RM, van Heiden PD, Gey van Pittius NC. Pulmonary infection due to the dassie bacillus (Mycobacterium tuberculosis complex sp.) in a free-living dassie (rock hyrax-Procavia capensis) from South Africa. Tuberculosis 2008; 88: 80-83.

31. Shaughnessy LJ, Smith LA, Evans J, Anderson D, Caldow G, Marion G, Low JC, Hutchings MR. High prevalence of paratuberculosis in rabbits is associated with difficulties in controlling the disease in cattle. Vet J 2013; 198: 267-270.

32. Smith N. The 'Dassie' bacillus. Tubercle 1960; 41: 203-212.

33. Sohal JS, Arsenault J, Labrecque O, Fairbrother JH, Roy JP, Fecteau G, L'Homme Y. Genetic Structure of Mycobacterium avium subsp paratuberculosis Population in Cattle Herds in Quebec as Revealed by Using a Combination of 
Multilocus Genomic Analyses. Journal of Clinical Microbiology 2014; 52: 2764-2775.

34. Vansnick E, Vercammen F, Bauwens L, D'Haese E, Nelis H, Geysen D. A survey for Mycobacterium avium subspecies paratuberculosis in the Royal Zoological Society of Antwerp. Vet J 2005; 170: 249-256.

35. Wagner JC, Bokkenheuser V. The mycobacterium isolated from the dassie Procavia capensis (Pallas). Tubercle 1961; 42: 47-56.

36. Wagner JC, Buchanan G, Bokkenheuser V, Leviseur S. An acid-fast bacillus isolated from the lungs of the Cape hyrax, Procavia capensis (Pallas). Nature 1958; 181: 284-285.

37. Whitlock RH, Buergelt C. Preclinical and clinical manifestations of paratuberculosis (including pathology). Vet Clin North Am Food Anim Pract 1996; 12: 345-356.

38. Wiid RE, Butler HJ. Population management of rock hyraxes (Procavia capensis) in residential areas. Pest Manag Sci 2015; 71: 180-188. 


\title{
CHAPTER IV
}

\section{Paper published}

\author{
Plos One
}

\section{Development of a recombinase polymerase amplification assay for rapid detection of Mycobacterium avium subsp. paratuberculosis}

Sören Hansen ${ }^{1}$, Jenny Schäfer ${ }^{1}$, Kim Fechner $^{1}$, Claus-Peter Czerny ${ }^{1}$, Ahmed Abd El Wahed $^{1 *}$

${ }^{1}$ Division of Microbiology and Animal Hygiene, Institute of Veterinary Medicine, Department of Animal Sciences, Faculty of Agricultural Sciences, Georg-August University, Goettingen, Germany

Short title: Rapid detection of Mycobacterium avium subsp. paratuberculosis *Corresponding author: E-mail: abdelwahed@gwdg.de and abdelwahed@me.com 


\section{Abstract}

Background: The detection of Mycobacterium avium subsp. paratuberculosis (MAP) infections in ruminants is crucial to control spread among animals and to humans. Cultivation of MAP is seen as the gold standard for detection, although it is very time consuming and labour intensive. In addition, several PCR assays have been developed to detect MAP in around 90 minutes, but these assays required highly sophisticated equipment as well as lengthy and complicated procedure.

Methodology/Principal Findings: In this study, we have developed a rapid assay for the detection of MAP based on the recombinase polymerase amplification (RPA) assay targeting a MAP specific region, the IS900 gene. The detection limit was 16 DNA molecules in 15 minutes as determined by the probit analysis on eight runs of the plasmid standard. Cross reactivity with other mycobacterial and environmentally associated bacterial strains was not observed. The clinical performance of the MAP RPA assay was tested using 48 MAP-positive and 20 MAP-negative blood, sperm, faecal and tissue samples. All results were compared with reads of a highly sensitive real-time PCR assay. The specificity of the MAP RPA assay was 100\%, while the sensitivity was $89.5 \%$.

Conclusions/Significance: The RPA assay is quicker and much easier to handle than real-time PCR. All RPA reagents were cold-chain independent. Moreover, combining RPA assay with a simple extraction protocol will maximize its use at point of need for rapid detection of MAP.

Keywords: Mycobacterium avium subsp. paratuberculosis; recombinase polymerase amplification assay; point of need 


\section{Introduction}

Paratuberculosis (Johne's disease) is caused by the Gram-positive, aerobic, non-motile, non-spore-forming and acid fast Mycobacterium avium subsp. paratuberculosis (MAP) [1]. The disease is characterized by a chronic progressive course marked by emaciation and accompanying lethal enteritis. MAP infects primarily large and small ruminants leading to diarrhea, weight loss and decreased milk production [2]. Particularly, animals infected subclinically represent a hotspot for transmitting MAP within the herd [3]. In humans, MAP was isolated from patients with Crohn's disease (inflammatory bowel disease) [4-6] and for more than 100 years, MAP has been discussed to be the causative agent [7]. Johne's disease is leading to huge economic losses in dairy production and since no pharmacological treatment or a licensed vaccine are available, early detection of the source of a MAP infection in a herd is very crucial to diminish such losses.

The gold standard for the diagnosis of MAP is culturing the bacteria [8], which takes up to 12 weeks and is only possible in highly equipped laboratories. Direct detection of MAP antibody with ELISA has been extensively applied but the clinical sensitivity and specificity is lower than the molecular assays [8]. Many real-time, conventional, seminested and nested PCR assays have been developed for the detection of MAP in up to 3 hours [9-11]. Nevertheless, a big challenge is the implementation of PCR in mobile point of need systems, because of its rapid thermocycling between the denaturation temperature, $95^{\circ} \mathrm{C}$, and approximately $50^{\circ} \mathrm{C}$ for primer annealing together with a precise temperature control [12].

Unlike PCR, isothermal DNA amplification assays do not need a controlled thermal cycling complex device. Moreover, these assays offer greater utility in the field by including simplistic reactor designs or portable heat sources [13]. Recombinase polymerase amplification (RPA) is an isothermal technology, which amplifies DNA at a constant temperature between $25^{\circ} \mathrm{C}$ and $42^{\circ} \mathrm{C}$ [13]. RPA depends on a combination of recombinase, single strand binding protein and strand displacing DNA polymerase for the DNA amplification step. Real-time detection of RPA amplicons relies on the exonuclease enzyme, which cuts at the basic mimic site presence between fluorophore and quencher in the RPA exo-probe. The RPA is extremely fast (3-15 minutes) and all reagents are cold chain independent. There have been developed several RPA assays to detect a wide range of different pathogens such as group B streptococci, Brucella or Mycobacterium tuberculosis [13-15]. 
In this study, a real-time RPA assay detecting MAP-DNA was developed. The assay sensitivity, specificity and cross reactivity were determined. The clinical performance of the MAP RPA assay was evaluated by 48 MAP-positive and 20 MAP-negative blood, sperm, faecal and tissue samples. All results were compared by a well-established realtime PCR [16].

\section{Materials and Methods}

\section{Ethical statement}

In total, the study included 68 archived DNA samples, which have been collected during routine veterinary examination in the Institute of Veterinary Medicine, Goettingen. All samples were taken under consideration of the German codex "Gute Veterinärmedizinische Praxis".

\section{Generation of a molecular and genomic DNA MAP Standards by PCR}

MAP (ATCC 19698) was ordered from Leibniz Institute DSMZ-German Collection of Microorganisms and cell cultures (DSMZ, Braunschweig, Germany, ID: 44133). DNA was extracted as follows: after culturing MAP on Herrold's Egg Yolk Agar Slants medium containing mycobactin and amphotericin B, nalidixinacid, vancomycin (BD, Franklin Lakes, NJ, USA) for 5 weeks, pure colonies were suspended in $100 \mu 1$ water and incubated at $99^{\circ} \mathrm{C}$ to $100^{\circ} \mathrm{C}$ with shaking at $300 \mathrm{rpm}$ in a Bioer Mixing Block MB102 (Bioer Technology, Hangzhou, China). After 20 minutes, the suspension was centrifuged at $14000 \mathrm{~g}$ for 10 minutes. Then the supernatant was collected and centrifuged at $14000 \mathrm{~g}$ for 10 minutes. The amount of DNA in the supernatant was measured by a NanoDrop ND-1000 spectrometer (Thermo Scientific, Waltham, MA, USA). A genomic DNA standard containing $10 \mathrm{ng} / \mu \mathrm{l}$ to $10 \mathrm{fg} / \mu \mathrm{l}$ of DNA dissolved in water was prepared to test the analytical sensitivity of the RPA assay. To generate the plasmid standard, a $587 \mathrm{bp}$ fragment was amplified covering the nucleotides 183 to 769 of MAP Gene IS900 sequence (Genbank accession number: AF416985.1) using the published primer sequences 5'-GTCGGCGTGGTCGTCTGCTGGGTTGAT-3 as a forward primer (FP) and 5'-GCGCGGCACGGCTCTTGTTGTAGTC-3 as a reverse primer (RP) [17]. The PCR reaction was performed on a T3000 thermocycler (Biometra $\mathrm{GmbH}$, Goettingen, Germany) using the following reaction mix: $11.8 \mu \mathrm{l}$ molecular 
biology H2O, $2 \mu 1$ BSA (Carl Roth GmbH + Co. KG, Karlsruhe, Germany), $2 \mu 1$ 10x Standard Reaction Buffer containing MgCl2 (Biotools B\&M Labs. S.A.,Madrid, Spain), $0.4 \mu \mathrm{l}$ DMSO (Carl Roth GmbH + Co. KG, Karlsruhe, Germany), $0.4 \mu$ l of each primer (10 $\mu \mathrm{M}$ stock solution), $0.1 \mu \mathrm{l}$ Biotools DNA polymerase (Biotools B\&M Labs. S.A.,Madrid, Spain), $0.1 \mu \mathrm{l}$ of each of the $10 \mu \mathrm{M}$ aNTP, tNTP, cNTP and gNTP stock solutions (Carl Roth GmbH + Co. KG, Karlsruhe, Germany) and $2.5 \mu \mathrm{l}$ of the template. The PCR cycles were programmed as follows: initial denaturation step at $95^{\circ} \mathrm{C}$ for three minutes, then thirty cycles of $95^{\circ} \mathrm{C} / 30 \mathrm{sec}, 64^{\circ} \mathrm{C} / 60 \mathrm{sec}$ and $72^{\circ} \mathrm{C} / 60 \mathrm{sec}$ and final extension step at $72^{\circ} \mathrm{C}$ for eight minutes. The amplified fragment was then cloned using the pGEM-T Easy Vector Systems I kit (Promega, Madison, USA) according the manufacture instructions. The number of plasmids were calculated using an equation as previously described [18]. The plasmids were linearized using the Ncol-HF (New England Biolabs, Ipswich, MA, USA).

A dilution range of $10^{0}$ to $10^{6}$ molecules $/ \mu 1$ of the standard was prepared. Both the molecular plasmid and genomic DNA standard were tested using a published quantitative real-time PCR protocol ([16], patent number: EP2841596A1) as described below.

\section{Real-time PCR}

The real-time PCR assay was performed on a LightCycler 480 (Roche, Mannheim, Germany) using the LightCycler 480 Probes Master Kit (Roche, Mannheim, Germany) to amplify a 139 bp long fragment (nucleotides 523 to 636 of the Genbank accession number: AF416985.1). The Reaction volume contained $10 \mu 1$ Light Cycler 480 Probes Master mix, $0.5 \mu \mathrm{l}$ of each $10 \mu \mathrm{M}$ FP: 5'-TACCGCGGCGAAGGCAAGAC-3' and RP: 5'-CGGAACGTCGGCTGGTCAGG-3', $1 \mu \mathrm{l}$ of $10 \mu \mathrm{M}$ probe: 5'-FAMATGACATCGCAGTCGAGCTG-BHQ-1-3 and $3 \mu$ molecular biology $\mathrm{H}_{2} \mathrm{O}$ as well as $5 \mu \mathrm{l}$ of the DNA template. The LightCycler was programmed as follow: first ten minutes pre-incubation at $95^{\circ} \mathrm{C}$, then 45 cycles of $95^{\circ} \mathrm{C} / 15 \mathrm{sec}, 60^{\circ} \mathrm{C} / 30 \mathrm{sec}$ and $72^{\circ} \mathrm{C} / 35 \mathrm{sec}$ followed by a final cooling step at $40^{\circ} \mathrm{C}$ for $30 \mathrm{sec}$.

\section{Real-time RPA primers and exo-probes}

Three regions (RPA1, RPA2 and RPA3) flanking the IS900 gene sequence were tested to select an area generating the highest RPA assay sensitivity (S1 Fig). In total, 18 forward primers, 25 reverse primers and three exo-probes were tested. The exo-probe 
was produced by TIB MOLBIOL (Berlin,Germany), while all the primers were purchased from Eurofins MWG Synthesis GmbH (Ebersberg, Germany). RPA primers were purified by gel filtration and the exo-probe by HPLC.

\section{RPA assay conditions}

The MAP RPA assay was performed using the TwistAmp Exo "Improved Formulation" kit (TwistDx Ltd, Cambridge, UK) according to the manuals instruction. Briefly, 29.5 $\mu 1$ Rehydration buffer, $6.7 \mu 1 \mathrm{H} 2 \mathrm{O}, 2.1 \mu \mathrm{l}$ of $10 \mu \mathrm{M}$ of both FP and RP, $0.6 \mu 1$ of the 10 $\mu \mathrm{M}$ of the exo-probe and $5 \mu \mathrm{l}$ of the DNA template were added to a freeze dried reaction pellet. For the negative control water was used instead of the DNA template. The RPA reactions were incubated at $42^{\circ} \mathrm{C}$ for fifteen minutes using a Tubescanner (Twista, TwistDx Ltd, Cambridge, UK). A mixing step by vortexing was performed after 230 seconds of the incubation in order to improve the sensitivity of the assay. The FAM fluorescence signal intensities were visualized on the Tubescanner studio software (version 2.07.06, QIAGEN Lake Constance GmbH, Stockach, Germany). For signal interpretation, threshold and first derivative analysis were applied.

\section{Analytical Sensitivity and specificity}

To determine the analytical sensitivity of the RPA assay, the molecular standard $\left(10^{6}\right.$ to $10^{0}$ molecules $\left./ \mu \mathrm{l}\right)$ was tested eight times and the genomic DNA standard ( $10 \mathrm{fg} / \mu \mathrm{l}$ to 10 $\mathrm{ng} / \mu \mathrm{l})$ in triplicate in both RPA and real-time PCR. The threshold times and the CT values were plotted against the number of molecules detected. Semi-log non-regression analysis was calculated with PRISM (Graphpad Software Inc., San Diego, California) and probit analysis was performed by STATISTICA (StatSoft, Hamburg, Germany). The analytical specificity of the RPA assay was tested with DNA isolated from different bacteria strains listed in Table 1. All bacteria species except Mycobacterium bovis were provided by DSMZ and grown on media following the instructions given by the DSMZ. All mycobacteria were tested positive with $16 \mathrm{~S}$ ribosomal DNA PCR assay to ensure DNA extraction quality [19]. Mycobacterium bovis DNA (ATCC: 27289) was provided by Institute for molecular Pathogenesis, Friedrich Loeffler Institut, Federal Research Institute for Animal Health, Jena, Germany. 
Table 1: List of bacteria species and strains used for determining the cross reactivity of the RPA assay.

\begin{tabular}{ll}
\hline Name of Bacteria & DSMZ ID \\
\hline Mycobacterium bovis & N/A \\
Mycobacterium avium subsp. avium & 44156 \\
Mycobacterium kansii & 44162 \\
Mycobacterium marinum & 44344 \\
Mycobacterium fortuitum & 46621 \\
Mycobacterium avium subsp. silvaticum & 44175 \\
Mycobacterium avium intracellulare & 43223 \\
Mycobacterium phlei & 43239 \\
Mycobacterium gordonae & 43213 \\
Mycobacterium smegmatis & 43756 \\
Mycobacterium scrofulaceum & 43992 \\
Rhodoccus hoagie & 20307 \\
Escherichia coli & 30083 \\
Clostridium perfringens & 756 \\
Staphylococcus aureus & 799 \\
Listeria monocytogenes & 15675 \\
Streptococcus uberis & 20569 \\
Streptococcus agalactiae & 2134 \\
Enterococcus faecalis & 1103 \\
\hline
\end{tabular}

\section{Clinical samples}

Archived DNA of MAP positive blood $(n=14)$, sperm $(n=18)$, faecal $(n=12)$ and tissue $(\mathrm{n}=4)$ samples as well as 20 MAP-negative faecal samples were tested in both RPA and real-time PCR in triplicate as described above. DNA extraction was performed by QIAamp DNA Blood Mini Kit (QIAgen GmbH, Hilden, Germany) using the recommended modifications as described previously [16]. 


\section{Results}

To determine the analytical sensitivity, a dilution range of the molecular DNA MAP standard was tested in RPA using the primer-probe combinations for three different regions in the IS900 gene sequence of the MAP (S1 Fig). Both RPA1 and RPA2 assays did not produce exponential amplification curves. Start points of the fluorescence signal for $5 \times 10^{5}$ standard DNA were eight and seven minutes, respectively (S2 Fig). In contrast, for the primer combination $\mathrm{FP} 1+\mathrm{RP} 2+\mathrm{P} 3$ from $\mathrm{RPA} 3$ assay, the start point was between four and five minutes and the detected concentration of molecular plasmid standard was down to 50 DNA copies (Fig 1, Table 2). This combination was used for the further validation of the RPA assay. The data set of eight replicates of the molecular DNA standards of both RPA and real-time PCR assays was used in the semi-log and probit analyses. The time needed to reach the detection limit was below ten minutes in the RPA assay and 90 minutes in the real-time PCR assay (Fig 2). In probit analysis, the limits of detection with a $95 \%$ probability were 16 and one molecules of MAP plasmid standard DNA per microliter in RPA and real-time PCR assays, respectively (Fig 3). Using the genomic DNA, the limit of detection for RPA and real-time PCR assays were $500 \mathrm{fg} /$ reaction and $50 \mathrm{fg} /$ reaction, respectively (Fig. 4).

The MAP RPA assay was highly specific, as it did not detect the DNA of other mycobacteria and environmental bacteria listed in Table 1. In addition, twenty MAP negative faecal samples were negative in both RPA and real-time PCR. Out of 48 realtime PCR positive DNA samples, forty-three were positive for MAP detection in RPA (89.5\%, S1 Table). 
Table 2: RPA primers and exo-probe combination, yielding the highest analytical sensitivity in the MAP-RPA assay.

\begin{tabular}{ll}
\hline Name & Sequence $\left(\mathbf{5}^{\prime}\right.$ to $\left.\mathbf{3}^{\prime}\right)$ \\
\hline Probe.RPA3 & ACGCCGGTAAGGCCGACCATTACTGCATGGTQTFTAACGA \\
& CGACGCGCA \\
MAP.RPA3.FP1 & CGTGGACGCCGGTAAGGCCGACCATTACTGCATGG \\
MAP.RPA3.RP2 & CGCCGCAATCAACTCCAGCAGCGCGGCCTC \\
\hline QTF are sites of the quencher and fluorophore in the order quencher BHQ1-dt (Q), \\
Tetrahydrofuran $(\mathrm{T})$ and Fam-dT $(\mathrm{F})$.
\end{tabular}


A

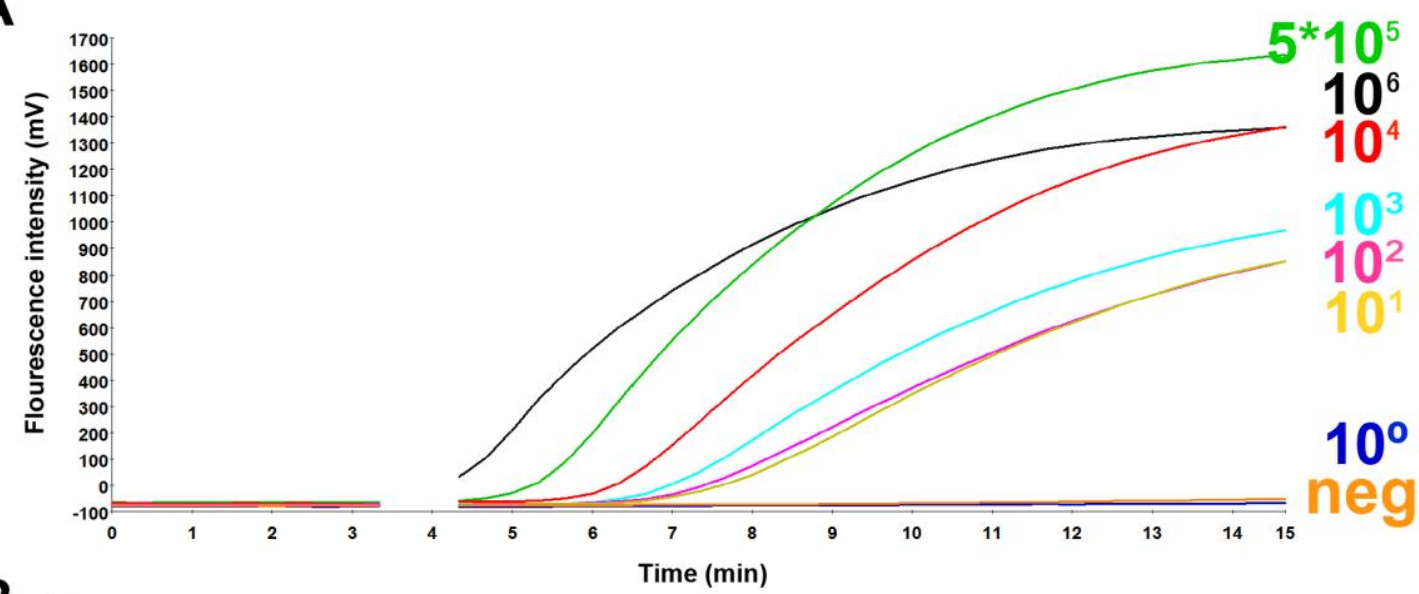

B

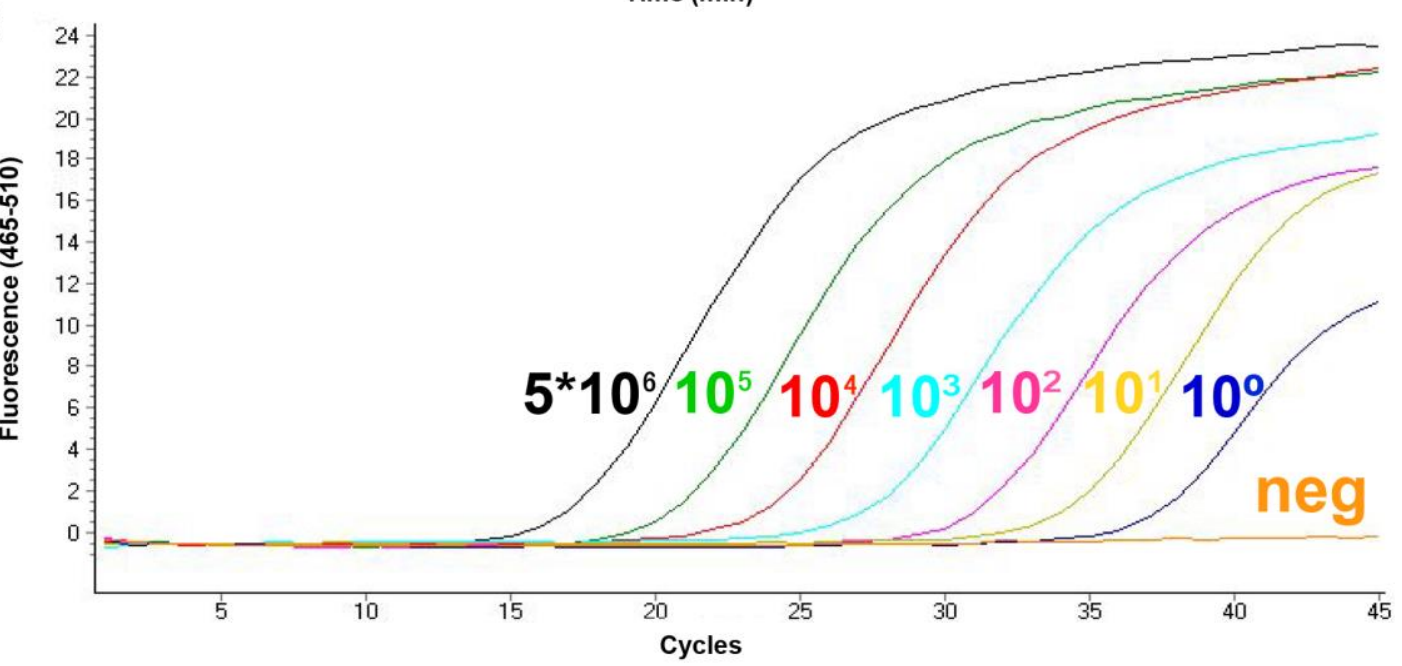

Fig. 1: Amplification curves in MAP RPA (A) and MAP real-time PCR (B) assays applying serial dilutions $\left(5 \times 10^{6}-1\right)$ of the molecular plasmid DNA standard. Analytical sensitivity of RPA and real-time PCR assays were 50 and 5 molecules/reaction, respectively. No fluorescent signals were detected in RPA after three minutes because the strip was taken out for mixing. 

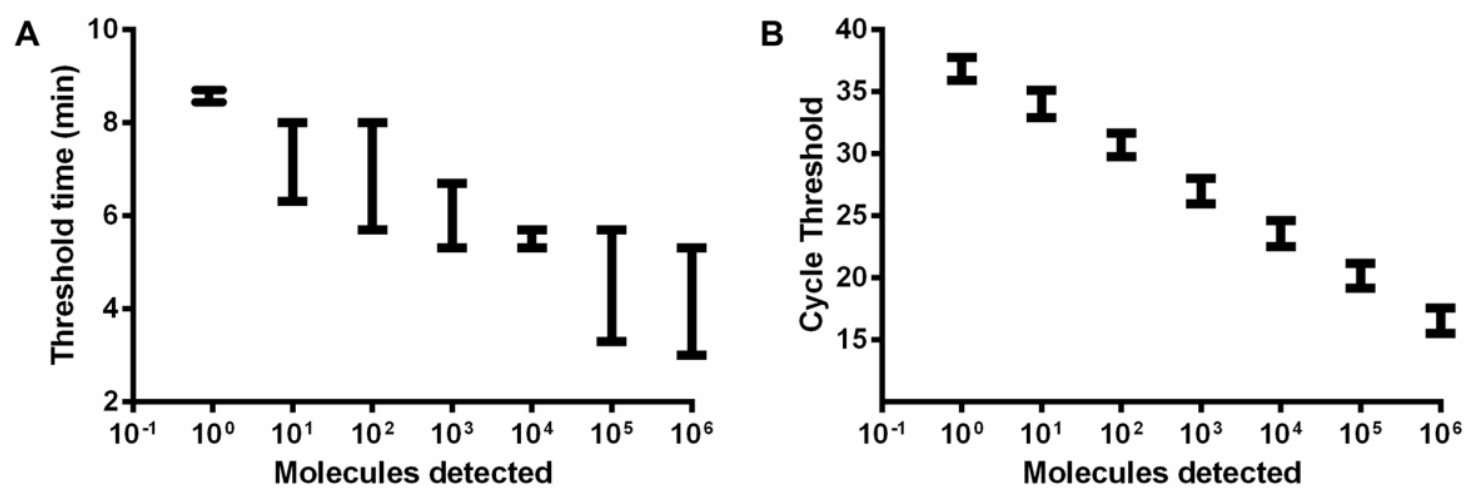

Fig. 2: Reproducibility of the RPA (A) and real-time PCR (B) assays using data set of eight runs of serial dilution of the MAP plasmid molecular standard in PRISM. RPA assay produced results between 2 to 10 minutes. In RPA $5 \times 10^{6}-10^{2}$ DNA molecules were detected in 8 out of 8 runs; 50, 7/8 and 5, 1/8 by the RPA assay. The error bars represent the range. The real-time PCR produced more linear results, due to the regular cycle format of the PCR, while there is no strict separation of the amplification cycles in the isothermal RPA technology.

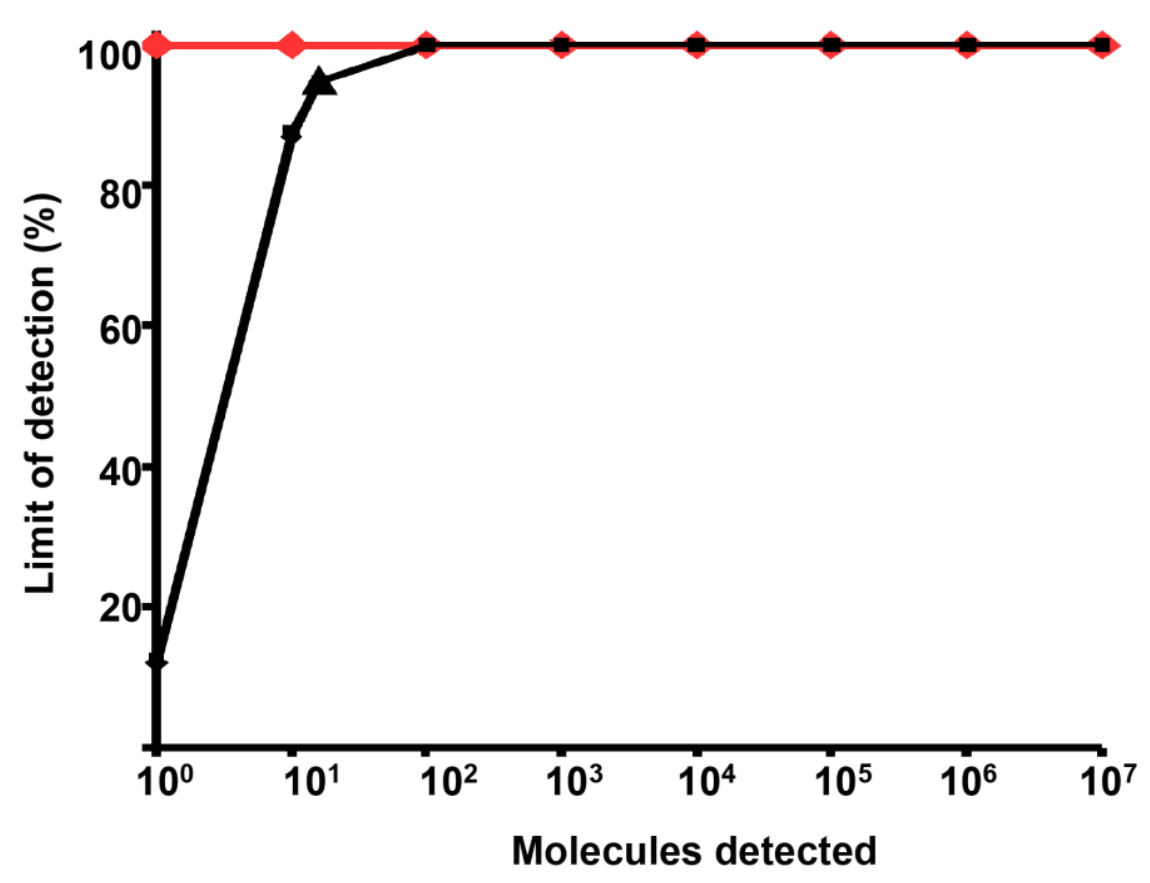

Fig. 3: Probit analylsis results for the MAP RPA (black) and real-time PCR (orange) assays using STATISTICA. Data sets of eight RPA and real-time PCR assay runs as showed in Fig. 1 was used. The limit of detection in RPA and real-time PCR at 95\% probability were 16 and one DNA molecules of the molecule plasmid DNA standard, respectively. 


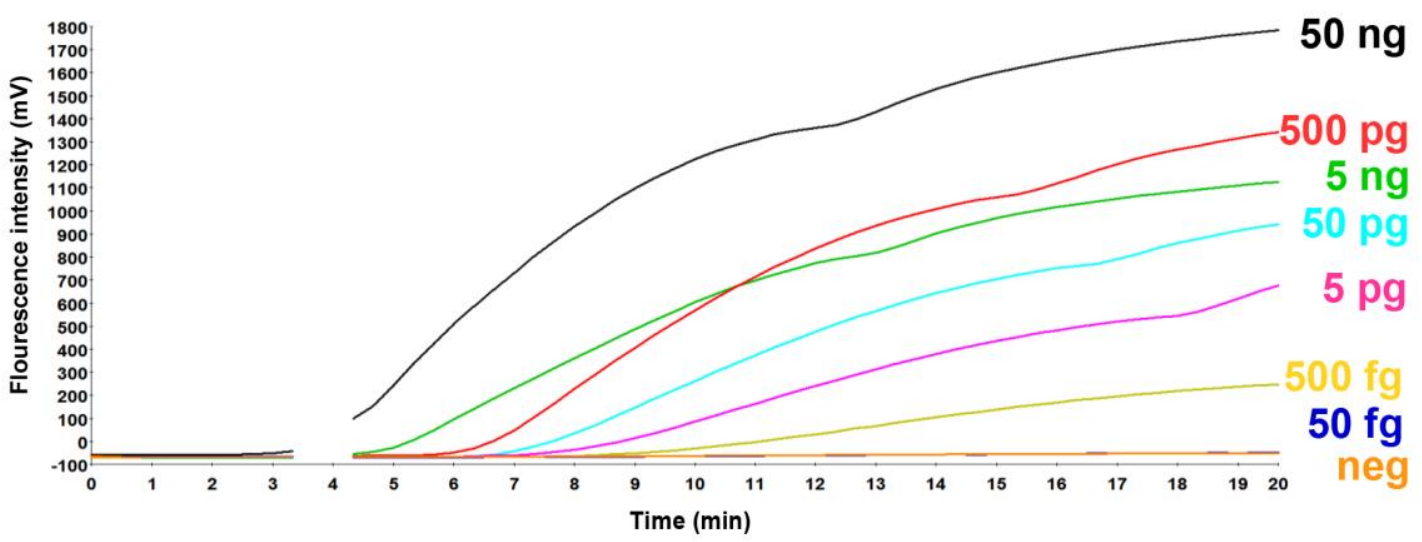

B

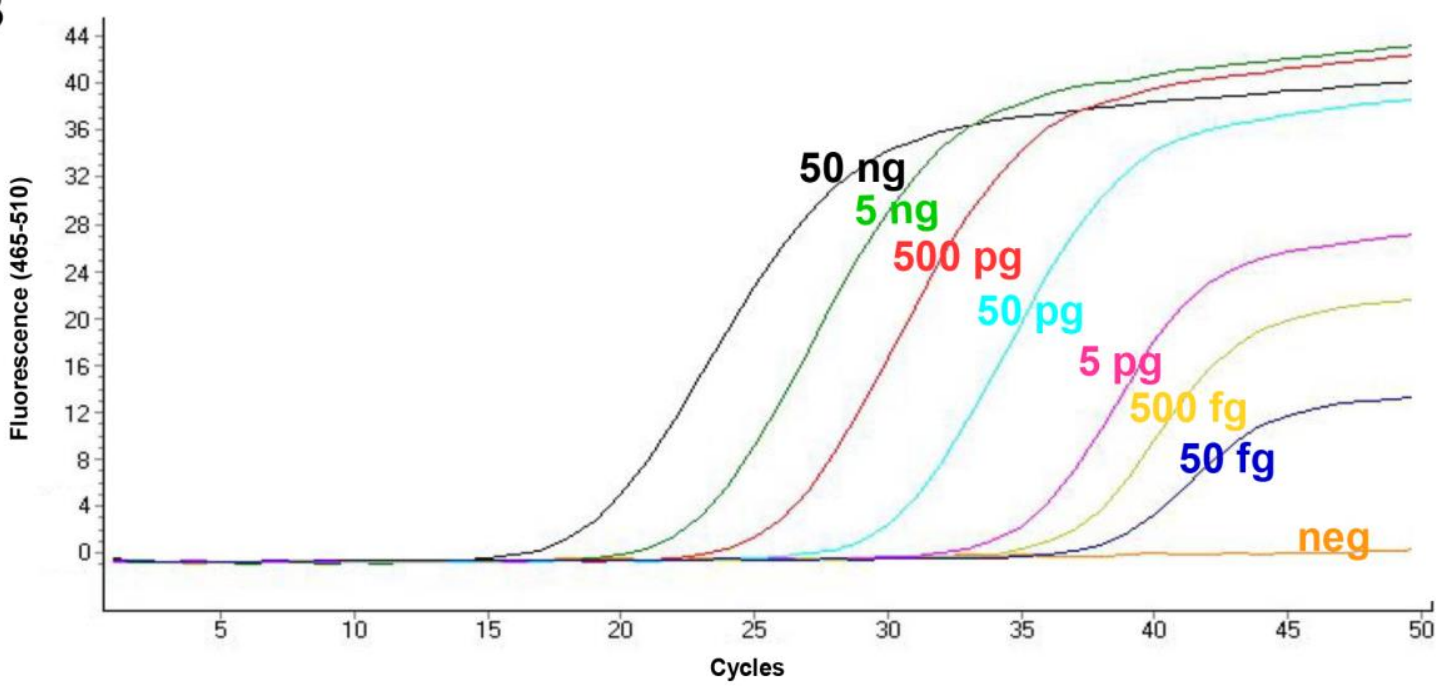

Fig. 4: Amplification curves in RPA (A) and real-time PCR (B) using the genomic MAP DNA standard isolated from MAP (ATCC 19698). The amount of the DNA in each dilution was calculated using NanoDrop. RPA detected down to $500 \mathrm{fg}$ DNA/reaction, while real-time PCR identified 50 fg DNA/reaction.

\section{Discussion}

In this study, we have developed a rapid RPA test detecting the IS900 gene sequence of MAP. The limit of detection using a molecular standard was 16 DNA molecules and $500 \mathrm{fg}$ by employing the genomic DNA. The latter corresponds to approximately 95 MAP genomes as one MAP genome has a weight of $5.29 \mathrm{fg}$ [16].

Three regions of the IS900 gene (RPA1, RPA2, RPA3, see S1 Fig) were tested in our setup. The RPA3 superposed the RPA1 and RPA2 assays in producing an exponential amplification curve and early fluorescence signal. The GC contents of RPA1, RPA2 and 
RPA3 regions were $70 \%$ and $67 \%$ and $55 \%$, respectively. The higher GC content of the target gene is able to slow down the performance of the strand exchanging proteins in the reaction [20, 21], which may explain the higher sensitivity of the RPA3 region containing lower GC content.

The clinical sensitivity of our assay was around $89.5 \%$ in comparison to real-time PCR upon using archived frozen DNA extracts. Although RPA is resistant to most PCR inhibitors [22], the presence of high amount of background DNA has a negative influence on the RPA sensitivity [23]. In contrast, false negative samples in our assay contain lower amount of total DNA than positive sample as determined by Nanodrop (S1 Table). The decrease in assay sensitivity due to low MAP DNA content in the sample was also excluded as two of the false negative samples had a high content of MAP DNA as determined by real-time PCR (CT: 26-29). In addition, the RPA assay detected four samples of high CT values (between 34 to 35).

Another point of need method named Loop-mediated isothermal amplification (LAMP) was applied to detect MAP DNA [24]. The LAMP assay turnout time was 100 minutes to detect $100 \mathrm{fg}$ of MAP genome. Moreover, six primers were employed to amplify the respective MAP gene. The results readout relies on the changes in the assay turbidity, which can be recognized by the naked eye or recorded in real-time by a portable device. In contrast, our RPA assay required 15 minutes process time, utilizes two primer and implemented a probe system to increase the assay specificity.

The MAP RPA assay was developed for the rapid and accurate detection of MAP DNA. A possible use of the RPA assay in combination with a fast DNA extraction protocol will allow its application for detection of MAP directly in the field. 


\section{List of Abbreviations}

\begin{tabular}{|c|c|}
\hline BHQ1 & Black Hole Quencher 1 \\
\hline $\mathrm{bp}$ & Base pair \\
\hline BSA & Bovine serum albumin \\
\hline $\mathrm{CT}$ & Cycle threshold \\
\hline dATP & Desoxyadenosintriphosphat \\
\hline $\mathrm{dCTP}$ & Desoxycytidintriphosphat \\
\hline dGTP & Desoxyguanosintriphosphat \\
\hline DMSO & Dimethylsulfoxid \\
\hline DNA & Deoxyribonucleic acid \\
\hline dTTP & Desoxythymidintriphosphat \\
\hline ELISA & Enzyme-linked Immunosorbent Assay \\
\hline FAM & 6-carboxyfluorescein \\
\hline fg & Femto gram \\
\hline Fig & Figure \\
\hline $\mathrm{FP}$ & Forward primer \\
\hline $\mathrm{g}$ & G-Force \\
\hline $\mathrm{H}_{2} \mathrm{O}$ & Water \\
\hline LAMP & Loop mediated isothermal amplification \\
\hline MAP & Mycobacterium avium subsp. paratuberculosis \\
\hline $\mathrm{MgCL}_{2}$ & Magnesium dichloride \\
\hline N/A & Not available \\
\hline ng & Nano gram \\
\hline PCR & Polymerase Chain Reaction \\
\hline $\mathrm{P}$ & Probe \\
\hline pg & Pico gram \\
\hline RPA & Recombinase Polymerase Amplification \\
\hline $\mathrm{RP}$ & Reverse primer \\
\hline $\mathrm{rpm}$ & Rounds per minute \\
\hline $\mathrm{sec}$ & Second \\
\hline TT & Time threshold \\
\hline$\mu 1$ & Micro liter \\
\hline
\end{tabular}




\section{Acknowledgement}

The authors would like to thank the Friedrich Loeffler Institut, Federal Research Institute for Animal Health, Jena, Germany for providing the Mycobacterium bovis DNA as well as Susanne Bölken-Fascher for her excellent technical assistance in the laboratory.

\section{References}

1. Manning EJ, Collins MT. Mycobacterium avium subsp. paratuberculosis: pathogen, pathogenesis and diagnosis. Rev Sci Tech. 2001;20(1):133-50. PubMed PMID: 11288509.

2. Chi J, VanLeeuwen JA, Weersink A, Keefe GP. Direct production losses and treatment costs from bovine viral diarrhoea virus, bovine leukosis virus, Mycobacterium avium subspecies paratuberculosis, and Neospora caninum. Prev Vet Med. 2002;55(2):137-53. PubMed PMID: 12350317.

3. Benedictus A, Mitchell RM, Linde-Widmann M, Sweeney R, Fyock T, Schukken $\mathrm{YH}$, et al. Transmission parameters of Mycobacterium avium subspecies paratuberculosis infections in a dairy herd going through a control program. Prev Vet Med. 2008;83(3-4):215-27. doi: 10.1016/j.prevetmed.2007.07.008. PubMed PMID: 17868937.

4. Mendoza JL, San-Pedro A, Culebras E, Cies R, Taxonera C, Lana R, et al. High prevalence of viable Mycobacterium avium subspecies paratuberculosis in Crohn's disease. World J Gastroenterol. 2010;16(36):4558-63. PubMed PMID: 20857526; PubMed Central PMCID: PMC2945487.

5. Knosel T, Schewe C, Petersen N, Dietel M, Petersen I. Prevalence of infectious pathogens in Crohn's disease. Pathol Res Pract. 2009;205(4):223-30. doi: 10.1016/j.prp.2008.04.018. PubMed PMID: 19186006.

6. Cheng J, Bull TJ, Dalton P, Cen S, Finlayson C, Hermon-Taylor J. Mycobacterium avium subspecies paratuberculosis in the inflamed gut tissues of patients with Crohn's disease in China and its potential relationship to the 
consumption of cow's milk: A preliminary study. World Journal of Microbiology \& Biotechnology 2005;21:1175-9.

7. Dalziel TK. Chronic Intestinal Enteritis. The British Medical Journal 1913;2.

8. Angelidou E, Kostoulas P, Leontides L. Bayesian estimation of sensitivity and specificity of a commercial serum/milk ELISA against the Mycobacterium avium subsp. Paratuberculosis (MAP) antibody response for each lactation stage in Greek dairy sheep. Prev Vet Med. 2016;124:102-5. doi: 10.1016/j.prevetmed.2015.12.011. PubMed PMID: 26754926.

9. Möbius P, Hotzel H, Rassbach A, Kohler H. Comparison of 13 single-round and nested PCR assays targeting IS900, ISMav2, f57 and locus 255 for detection of Mycobacterium avium subsp. paratuberculosis. Vet Microbiol. 2008;126(4):32433. doi: 10.1016/j.vetmic.2007.07.016. PubMed PMID: 17889455.

10. Vansnick E, De Rijk P, Vercammen F, Geysen D, Rigouts L, Portaels F. Newly developed primers for the detection of Mycobacterium avium subspecies paratuberculosis. Vet Microbiol. 2004;100(3-4):197-204. doi: 10.1016/j.vetmic.2004.02.006. PubMed PMID: 15145498.

11. Plain KM, Marsh IB, Waldron AM, Galea F, Whittington AM, Saunders VF, et al. High-throughput direct fecal PCR assay for detection of Mycobacterium avium subsp. paratuberculosis in sheep and cattle. J Clin Microbiol. 2014;52(3):745-57. doi: 10.1128/JCM.03233-13. PubMed PMID: 24352996; PubMed Central PMCID: PMCPMC3957769.

12. Lutz S, Weber P, Focke M, Faltin B, Hoffmann J, Muller C, et al. Microfluidic lab-on-a-foil for nucleic acid analysis based on isothermal recombinase polymerase amplification (RPA). Lab Chip. 2010;10(7):887-93. doi: 10.1039/b921140c. PubMed PMID: 20300675.

13. Boyle DS, McNerney R, Teng Low H, Leader BT, Perez-Osorio AC, Meyer JC, et al. Rapid detection of Mycobacterium tuberculosis by recombinase polymerase amplification. PloS one. 2014;9(8):e103091. doi: 10.1371/journal.pone.0103091. PubMed PMID: 25118698; PubMed Central PMCID: PMC4138011.

14. Daher RK, Stewart G, Boissinot M, Bergeron MG. Isothermal recombinase polymerase amplification assay applied to the detection of group B streptococci in 
vaginal/anal samples. Clin Chem. 2014;60(4):660-6. doi: 10.1373/clinchem.2013.213504. PubMed PMID: 24463560.

15. Ren H, Yang M, Zhang G, Liu S, Wang X, Ke Y, et al. Development of a rapid recombinase polymerase amplification assay for detection of Brucella in blood samples. Mol Cell Probes. 2016;30(2):122-4. doi: 10.1016/j.mcp.2016.02.007. PubMed PMID: 26911890.

16. Fechner K, Schäfer J, Wiegel C, Ludwig J, Münster $\mathrm{P}$, Sharifi AR, et al. Distribution of Mycobacterium avium subsp. paratuberculosis in a Subclinical Naturally Infected German Fleckvieh Bull. Transboundary and emerging diseases. 2015. doi: 10.1111/tbed.12459. PubMed PMID: 26671341.

17. Münster P, Völkel I, Wemheuer W, Petschenka J, Wemheuer W, Steinbrunn C, et al. Detection of Mycobacterium avium ssp. paratuberculosis in ileocaecal lymph nodes collected from elderly slaughter cows using a semi-nested IS900 polymerase chain reaction. Vet Microbiol. 2011;154(1-2):197-201. doi: 10.1016/j.vetmic.2011.06.033. PubMed PMID: 21775077.

18. Zhong C, Peng D, Ye W, Chai L, Qi J, Yu Z, et al. Determination of plasmid copy number reveals the total plasmid DNA amount is greater than the chromosomal DNA amount in Bacillus thuringiensis YBT-1520. PloS one. 2011;6(1):e16025. doi: 10.1371/journal.pone.0016025. PubMed PMID: 21283584; PubMed Central PMCID: PMC3026805.

19. Weisburg WG, Barns SM, Pelletier DA, Lane DJ. 16S ribosomal DNA amplification for phylogenetic study. J Bacteriol. 1991;173(2):697-703. PubMed PMID: 1987160; PubMed Central PMCID: PMCPMC207061.

20. Patil KN, Singh P, Muniyappa K. DNA binding, coprotease, and strand exchange activities of mycobacterial RecA proteins: implications for functional diversity among RecA nucleoprotein filaments. Biochemistry. 2011;50(2):300-11. doi: 10.1021/bi1018013. PubMed PMID: 21133394.

21. Daher R. Recombinase polymerase amplification technology: Assessment for nucleic acid-based point-of-care diagnostics. Québec, Canada: LAVAL University; 2015. 
22. Piepenburg $\mathrm{O}$, Williams $\mathrm{CH}$, Stemple DL, Armes NA. DNA detection using recombination proteins. PLoS Biol. 2006;4(7):e204. doi: 10.1371/journal.pbio.0040204. PubMed PMID: 16756388; PubMed Central PMCID: PMCPMC1475771.

23. Rohrman B, Richards-Kortum R. Inhibition of recombinase polymerase amplification by background DNA: a lateral flow-based method for enriching target DNA. Anal Chem. 2015;87(3):1963-7. doi: 10.1021/ac504365v. PubMed PMID: 25560368.

24. Enosawa M, Kageyama S, Sawai K, Watanabe K, Notomi T, Onoe S, et al. Use of loop-mediated isothermal amplification of the IS900 sequence for rapid detection of cultured Mycobacterium avium subsp. paratuberculosis. J Clin Microbiol. 2003;41(9):4359-65. PubMed PMID: 12958269; PubMed Central PMCID: PMCPMC193777. 

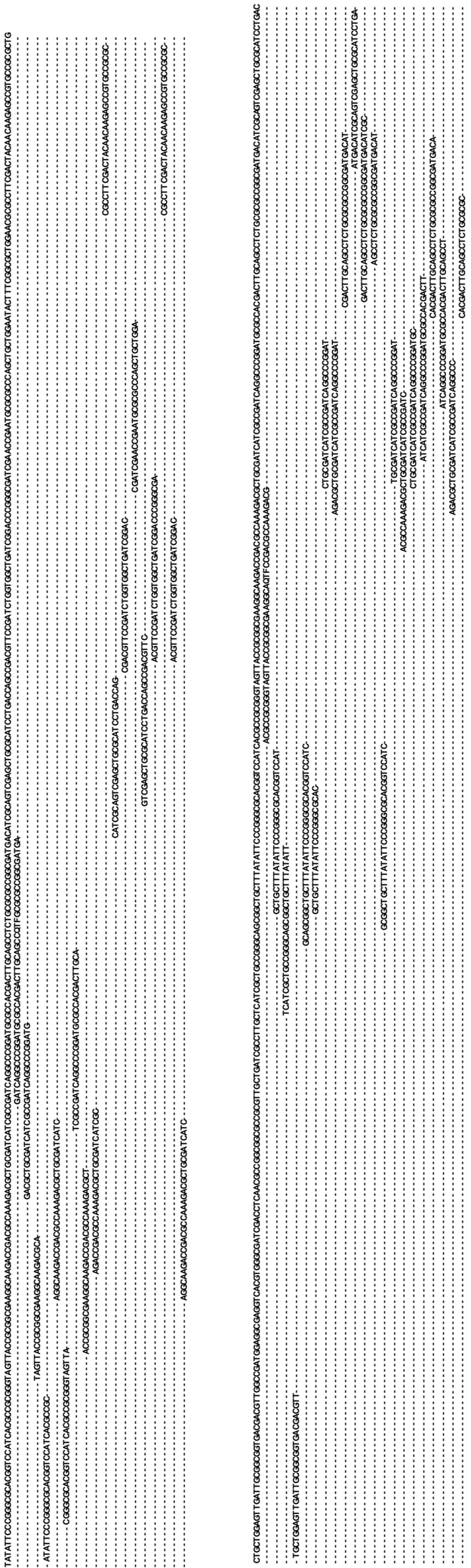

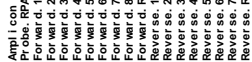

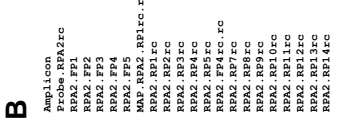

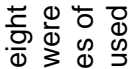

क्ष

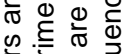

离

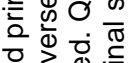

일

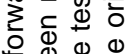

ه

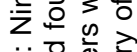

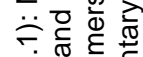

它

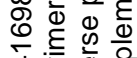

岁言㐫 亭

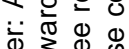

है을 के 뜽 은 응

के

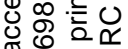

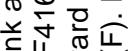

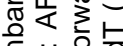

ه

बे है

它

के 응 물

s 80

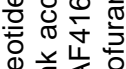

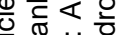

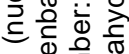

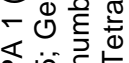

偪

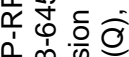

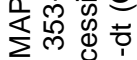

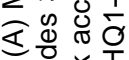

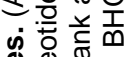

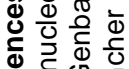

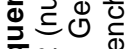

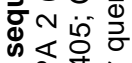

\&

운

응

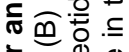

๘

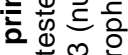

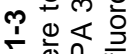

$\varangle \prod^{\infty}$

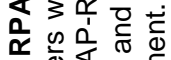

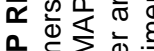

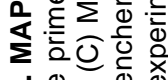

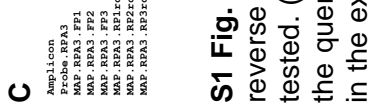


A

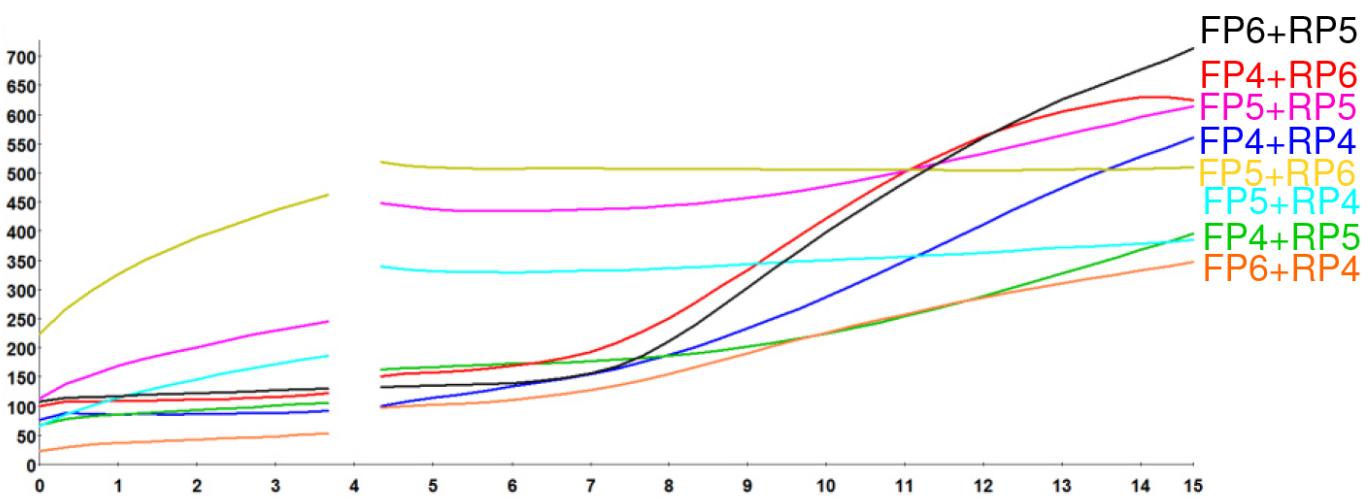

B

Time (min)

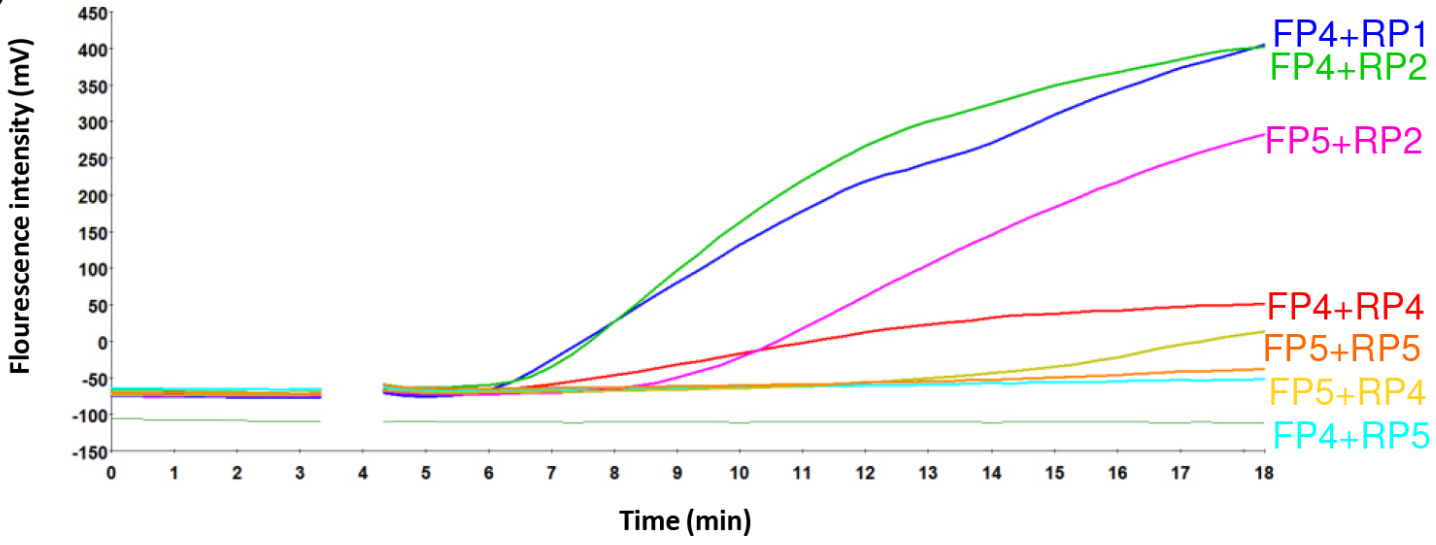

C

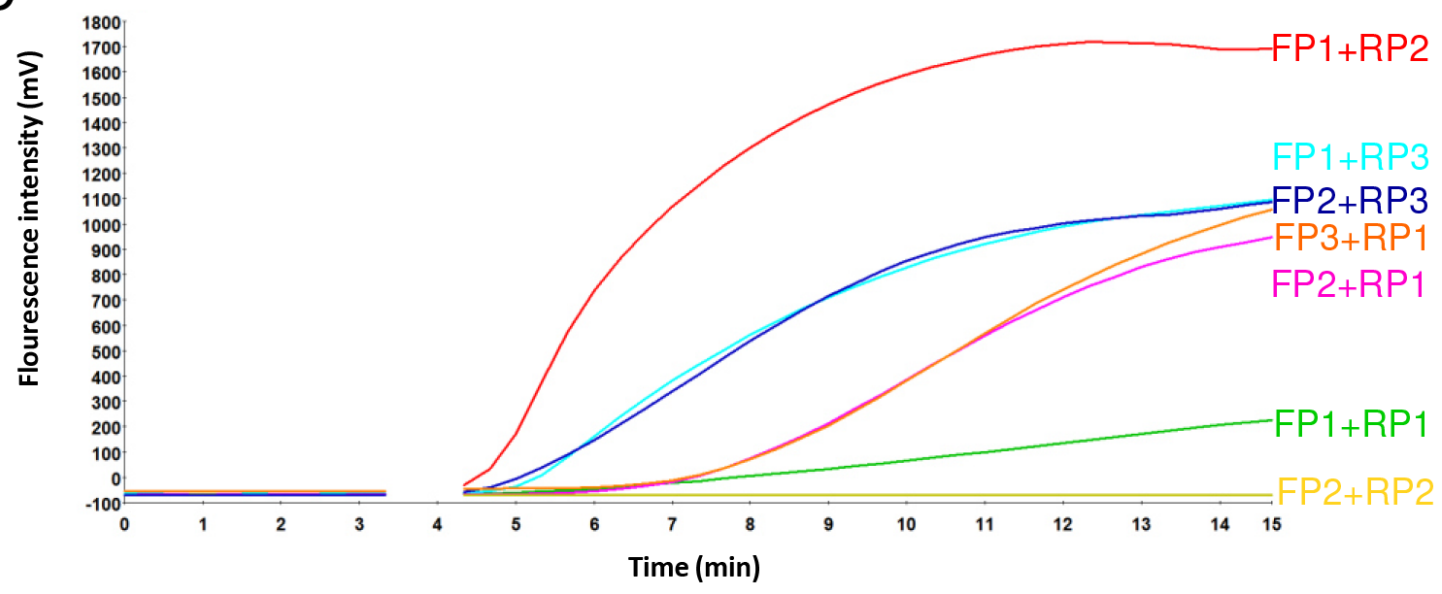

S2 Fig. Amplification curves of screening primers combinations targeting three RPA regions on the IS900 gene. A, RPA1; B, RPA2; C, RPA3. RPA3 FP1+RP2 produced the best exponential curve and a start point after 4 minutes. 
S1 Table. Results of screening MAP-positive DNA samples using both real-time PCR and RPA assays. All results are mean out of triplicate. CT is the cycle threshold and TT is the threshold time. The amount of DNA in each sample was calculated using NanoDrop.

\begin{tabular}{|c|c|c|c|c|}
\hline Sample ID & Matrix & $\begin{array}{l}\text { Real time } \\
\text { PCR }(\mathrm{CT})\end{array}$ & RPA (TT) & NanoDrop $(\mathrm{ng} / \mu \mathrm{l})$ \\
\hline 1 & feces & 30.74 & 6 & 15,11 \\
\hline 2 & blood & 32.81 & 10,5 & 40,34 \\
\hline 4 & feces & 32.24 & 6,5 & 11,22 \\
\hline 5 & feces & 30,00 & 6 & 21,95 \\
\hline 6 & blood & 32,25 & 6 & 3,92 \\
\hline 7 & sperm & 34,81 & neg & 33,43 \\
\hline 8 & sperm & 30,04 & 8.0 & 52,46 \\
\hline 9 & feces & 30,81 & 8.0 & 25,38 \\
\hline 10 & blood & 34,13 & 9.5 & 1,52 \\
\hline 11 & blood & 30,12 & 7.0 & 13,48 \\
\hline 12 & sperm & 29,92 & 8.0 & 19,12 \\
\hline 13 & sperm & 32,38 & 7.25 & 53,73 \\
\hline 14 & blood & 36,85 & neg & 12,49 \\
\hline 15 & blood & 32,48 & 7.5 & 10,49 \\
\hline 16 & sperm & 34,88 & 11.5 & 7,23 \\
\hline 17 & sperm & 35,02 & 10.6 & 14,68 \\
\hline 18 & feces & 33,42 & 7.0 & 98,92 \\
\hline 20 & blood & 33,24 & 6.0 & 25,28 \\
\hline 21 & blood & 29,93 & 7,5 & 33,95 \\
\hline 22 & blood & 28,92 & 5.5 & 6,03 \\
\hline 23 & blood & 29,28 & 6.0 & 0,64 \\
\hline 24 & blood & 28,92 & 6.5 & 1,99 \\
\hline 25 & sperm & 29,40 & 10.0 & 176,52 \\
\hline 26 & sperm & 28,92 & 8.0 & 148,90 \\
\hline 27 & blood & 31,52 & 6.5 & 7,68 \\
\hline 28 & sperm & 31,77 & 5.35 & 186,96 \\
\hline 29 & sperm & 32,05 & 4.65 & 175,92 \\
\hline 30 & sperm & 30,70 & 12.0 & 147,51 \\
\hline 31 & sperm & 29,90 & neg & 149,33 \\
\hline 32 & feces & 32,83 & 9.25 & 78,98 \\
\hline 33 & sperm & 26,93 & neg & 183,44 \\
\hline 34 & sperm & 27,33 & 3.5 & 511,83 \\
\hline 35 & feces & 22,56 & 14.25 & 92,72 \\
\hline 36 & feces & 22,94 & 5.5 & 62,11 \\
\hline 37 & feces & 19,03 & 4.75 & 75,88 \\
\hline 38 & feces & 23,55 & 5.0 & 56,11 \\
\hline 39 & blood & 20,58 & 11.0 & 11,53 \\
\hline 40 & blood & 24,41 & 11.0 & 12,52 \\
\hline 41 & tissue goat & 26,77 & 7.25 & 299,18 \\
\hline
\end{tabular}


S1 Table. continued

\begin{tabular}{ccccc}
\hline Sample ID & Matrix & $\begin{array}{c}\text { Real time } \\
\text { PCR }(\mathbf{C T})\end{array}$ & RPA (TT) & NanoDrop $(\mathbf{n g} / \boldsymbol{\mu l})$ \\
\hline $\mathbf{4 2}$ & tissue goat & 22,65 & 3.5 & 235,80 \\
$\mathbf{4 3}$ & tissue goat & 31,73 & 14,50 & 98,62 \\
$\mathbf{4 4}$ & tissue goat & 33,91 & 8,00 & 79,57 \\
$\mathbf{4 5}$ & sperm & 32,63 & 9.65 & 414,81 \\
$\mathbf{4 6}$ & sperm & 32,16 & 7.5 & 385,90 \\
$\mathbf{4 7}$ & sperm & 32,01 & neg & 286,59 \\
$\mathbf{4 8}$ & sperm & 32,14 & 10,00 & 250,48 \\
$\mathbf{4 9}$ & feces & 35,26 & 6,00 & 130,73 \\
$\mathbf{5 0}$ & feces & 27,05 & 7,00 & 63,70 \\
\hline
\end{tabular}




\section{AUTHOR'S CONTRIBUTIONS}

Chapter I: Distribution of Mycobacterium avium subsp. paratuberculosis in a Subclinical Naturally Infected German Fleckvieh Bull

The author (KF) established the matrix-specific adaptation for DNA purification und cultural preparation, investigated faecal, semen, blood and tissue samples, performed sequencing, analysed the sequences, and was the primary writer of the manuscript.

Chapter II: Detection of Mycobacterium avium subsp. paratuberculosis in nonhuman primates

The author (KF) established the matrix-specific adaptation for DNA purification und culture preparation, investigated faecal and tissue samples, performed sequencing, analysed the sequences, and was the primary writer of the manuscript.

Chapter III: Detection of Mycobacterium avium subsp. paratuberculosis in captured wildlife rock hyraxes (Procavia capensis)

The author (KF) analysed all results of the PCR, culture and ELISA experiments, and was the primary writer of the manuscript.

Chapter IV: Development of a recombinase polymerase amplification assay for rapid detection of the Mycobacterium avium subsp. Paratuberculosis

The author $(\mathrm{KF})$ supported the data analyses and the scientific writing process. 
GENERAL DISCUSSION

3 GENERAL DISCUSSION 


\section{DISCUSSION}

Mycobacterium avium subspecies paratuberculosis (MAP) is the aetiological agent of paratuberculosis, a disease found worldwide that mainly affects ruminants. In the agricultural sector, this disease causes significant financial losses, especially for the dairy industry. Furthermore, the involvement of MAP in the pathological process of Crohn's disease in humans has been controversially discussed for many years. It is assumed that the consumption of animal products such as milk, cheese, and meat are possible vectors of MAP transmission from animals to humans. The potential zoonotic risk and the economic losses in the agricultural sector are two main reasons for the indispensability of effective control of the pathogen. The long incubation period of paratuberculosis without visible clinical signs leads to the unrecognized spread of the bacteria. Unfortunately, the current diagnostic possibilities are limited, especially during the subclinical stage of the disease. In particular, an incomplete understanding of the pathogenesis and immunology during this stage of the disease leads to diagnostic uncertainties.

\section{Detection pattern of MAP in a subclinically infected bull}

This thesis (chapter I) provides detailed information about the occurrence of MAP in a naturally infected, but clinically unremarkable, bull from the age of seven weeks until necropsy (a period of four years). In the longitudinal study performed, the detectability of MAP in faeces, semen, and blood was periodically investigated by PCR and bacterial culture. The immune response against MAP was measured by a commercially available antibody ELISA. The results of this study clearly demonstrate the difficulties involved in MAP diagnosis during the subclinical stage of the disease. MAP was only intermittently present in one, two or all three investigated matrices. On $65 \%$ of the sample days (up to nine weeks), the pathogen was undetectable by both PCR and bacterial culture. The detectability of the pathogen was not predictable by either PCR or bacterial culture. As outlined in the general introduction (Section: Control measures in Europe), most control programs in Europe are based on the detection of an antibody response against MAP in milk or blood by commercially available ELISA. This method is used due to the low costs and the feasibility of investigating large sample numbers. However, although the bull excreted MAP in concentrations of up to $6.25 \times 10^{6}$ genome 
equivalents/g faeces, an antibody response against MAP was not measured during the entire investigation period. Animals without a measurable antibody response spread the pathogen unrecognized within and between the herd, and may be one reason for the reduced success of paratuberculosis control programs. In agreement with our results, a recent study showed that unpredictable monthly variations exist in the test results of cows using a commercial milk ELISA, solid and broth faecal culture, and faecal realtime PCR (Laurin et al., 2017). To date, the exact mechanisms involved in the pathogenesis and immune response during the subclinical stage of the disease remain incomplete. The resulting reduced efficiency of diagnostic assays leads to the unrecognized spread of MAP. Therefore, the development of an efficient diagnostic strategy to identify subclinically infected animals should remain a priority.

\section{Distribution of MAP within subclinically infected animals}

Clinical symptoms develop in only 10-15\% of MAP-infected cattle within one to ten years after infection (Olsen et al., 2002). Most of the infected animals remain in the subclinical disease stage. Animals in this stage do not show clinical signs; however, they do intermittently excrete the pathogen (Olsen et al., 2002). This investigation (chapter I) was conducted to provide detailed information about the distribution of MAP within a naturally infected, but clinically unremarkable bull. The examination of the collected tissues demonstrated that MAP can be detected by PCR in tissues of the lymphatic system (7/15), digestive tract (5/14), and the urogenital tract (5/9), with concentrations of up to $3.9 \times 10^{6} \mathrm{MAP}-\mathrm{GE} / \mathrm{g}$ tissue. Although MAP was widely spread within the host, neither macroscopic nor histological lesions, characteristic of a MAP infection, were observed. In general, such a bland pathological picture in PCR-positive samples is an uncommon finding in MAP-infected ruminants. However, another study also reported no macroscopic lesions or clinical signs after confirming the presence of MAP in many tissues of the cattle (Brady et al., 2008). Therefore, it can be assumed that MAP can replicate within the asymptomatic host without triggering pathological changes. While paratuberculosis in domestic ruminants is intensively researched, little is known about the clinical signs and/or pathological and histological changes of MAP infection in non-ruminants. MAP has previously been detected in many non-ruminate animal species, such as foxes (Vulpes vulpes), stoats (Mustela erminea), weasels (Mustela nivalis), crows (Corvus corone), and wood mice (Apodemus sylvaticus) (Beard 
et al., 2001a; Carta et al., 2013; Greig et al., 1999). Although the pathogen has been found in these animals, no or only mild gross pathological changes and histopathological lesions were often found. In the present investigation (chapters II and III), MAP was detected in the tissues of rock hyraxes (Procavia capensis) and in two non-human primate species (cottontop tamarin, Saguinus ooedipus and common marmoset, Callithrix jacchus). The pathogen was detected in these animals by PCR, but typical clinical signs, and macroscopic or microscopic pathological changes were not observed. As demonstrated in the investigated bull, MAP is replicated and excreted via faeces without triggering typical macroscopic or microscopic changes (chapter I). It could therefore be assumed that MAP may also replicate in non-ruminates without eliciting characteristic lesions. Therefore, infected non-ruminants should also be considered a source of pathogen spread.

It was striking that MAP DNA was detected in the bone marrow of the German Fleckvieh bull (chapter I) and the common marmoset (chapter II). MAP was detected in only one previous study in the bone marrow of two stumptail macaques (Macaca arctoides) by microscopic examination (McClure et al., 1987). A recent study indicated that a MAP-related pathogen, Mycobacterium tuberculosis, persists within the $\mathrm{CD} 271^{+} / \mathrm{CD} 45^{-}$mesenchymal stem cells of the bone marrow in a viable but nonculturable (VBNC) state (Das et al., 2013). The VBNC state is assumed to be a potential survival strategy of MAP (Lamont et al., 2012; Whittington et al., 2004). New data suggest that sporulation-like mechanisms may be a strategy used by MAP to accomplish persistence in the host and the surrounding environment (Lamont et al., 2012). VBNC bacteria likely adapt to less optimal living and reproduction conditions through a wide range of metabolic modifications, including changes in the cell wall, or down-regulation of DNA replication or cell division (Lamont et al., 2012; Mukamolova et al., 2003; Rowe and Grant, 2006; Whittington et al., 2004). Although bacteria in the VBNC state fail to grow by standard bacteriological methods, they remain alive (Oliver, 2010). Furthermore, mesenchymal stem cells found in bone marrow appear to play a key role in the establishment of latent tuberculosis (Das et al., 2013; Raghuvanshi et al., 2010). Hence, the bone marrow may also provide a niche for latent infections with MAP in ruminants as well as non-ruminants. To investigate the occurrence of MAP in the bone marrow, further studies should include an examination of MAP in this tissue, in addition to gastrointestinal tissues and lymph nodes. 


\section{Transmission of MAP via the faeces of calves}

For the effective control of paratuberculosis, it is necessary to consider all possible routes of transmission. A central factor in the transmission of MAP is the infection of newborn calves. These animals are usually infected shortly after their birth via the faecal-oral route. Colostrum and milk of subclinically (Streeter et al., 1995) as well as clinically infected cattle (Taylor et al., 1981) can contain the pathogen, but this is usually in low numbers (Grant et al., 2001). Faecally contaminated colostrum, milk and food are the main sources of infection. In addition to asymptomatic, infected adult animals, experimentally infected calves younger than eight months also sporadically excrete MAP in faeces (Collins and Zhao, 1994; McDonald et al., 1999). The results of the present study (chapter I) confirm the repeated excretion of MAP in a naturally infected calf between the ages of seven and 16 weeks. Over the entire investigation period (four years), the highest MAP genome equivalent concentration $\left(6.25 \times 10^{6} / \mathrm{g}\right)$ was measured in the faeces of the bull calf. Such high concentrations of MAP may be high enough to elicit an infection in another susceptible animal, as a concentration of $10^{3} \mathrm{MAP} / \mathrm{animal}$ is estimated to be sufficient to infect calves (Brotherston et al., 1961). However, measuring the concentration of MAP based on the results of the performed real-time PCR and a differentiation between viable and dead MAP cells is not possible with this method. Furthermore, the differentiation between a true infection (active MAP shedding) and a pass through (passive MAP shedding) of MAP is difficult to ascertain, especially for calves (van Roermund et al., 2007). True infection of the investigated bull calf was confirmed at the age of 40 weeks by initial detection of MAP DNA in the blood. The detection of MAP within the blood indicates that the pathogen crosses the intestinal mucosa, establishing a true infection. The possible risk of calves as a source of infection underlines the importance of immediate separation of calf and cow after birth. This separation is one of the key measures that will prevent the transmission of MAP from cow to calf and in turn the transmission from calf to calf (Kirkeby et al., 2016), whether they are truly infected or simply pass through the pathogen.

\section{Sexual transmission of MAP}

In addition to the faecal-oral infection, it is well known that MAP can be transmitted vertically from subclinically as well as clinically infected cows in utero to the foetus 
(Pribylova et al., 2013). However, the role of bulls in the spread of MAP has been poorly explored. Since 1970, it has been known that MAP is detectable in the reproductive organs and semen of bulls (Larsen and Kopecky, 1970). To date, only a few studies have been performed on the occurrence of MAP in bulls. The results of all performed studies were listed in Table 7 and 8, demonstrating the occurrence of MAP in bull reproductive organs and semen. Nevertheless, the role of bulls in the vertical transmission of MAP via semen remains unanswered.

Therefore, another objective of the present thesis (chapter I) was to monitor the excretion of MAP-contaminated semen and the distribution of MAP within the male reproductive organs of an infected, but asymptomatic bovine host. MAP DNA was detected in only four of the 89 semen samples $(5 \%)$ by snPCR. This failure of detection and/or quantification by qPCR and bacterial culture indicated the presence of only low numbers of MAP. In general, it has been argued that the transmission of MAP is unlikely, due to low numbers of MAP in semen (Buergelt and Williams, 2004; Philpott, 1993). However, in a longitudinal study of another asymptomatically infected Fleckvieh bull, MAP DNA was repeatedly detectable in concentrations of up to $5.7 \times 10^{5}$ MAP genome equivalents/ml (Münster et al., 2013b). It is not clear if such concentrations of MAP in semen are enough to elicit an infection. In addition to the monitoring of MAP contamination in semen, the presence of MAP within the male reproductive organs was screened in this investigation (chapter I). In the present study, the pathogen was detected in five of nine urogenital tract tissue samples, including the testicle, epididymis, ampulla of deferent duct, prostate gland, and kidney. In contrast to the investigated bull, all other previously examined bulls showed clinical symptoms, or if they were asymptomatic, typical macroscopic abnormalities were found during necropsy, indicating an advanced disease stage (Ayele et al., 2004; Glawischnig et al., 2004; Khol et al., 2010; Larsen and Kopecky, 1970; Larsen et al., 1981). The detection of MAP in the semen as well as in the reproductive organs supports the hypothesis that asymptomatic bulls likely transmit MAP via semen, especially under natural mating conditions. In cases of artificial insemination, the influence of antibiotics in various semen extenders as well as the freezing process on the viability of MAP in semen should be tested. To monitor the occurrence of viable MAP in native and diluted semen of subclinically as well as clinically infected breeding bulls, the development of a sensitive and specific method is necessary. One promising method for the detection of 
viable MAP may be a phage-based assay, due to a less time-consuming detection in comparison to culture-based approaches. The phage assay has been adapted for the detection of MAP in many different matrices, such as milk and blood (Botsaris et al., 2016; Foddai et al., 2011; Foddai and Grant, 2017; Stanley et al., 2007; Swift et al., 2013); however, it has not yet been adapted for the detection of MAP in semen.

Surprisingly, MAP was also detectable by PCR in the reproductive organs of one female and one male rock hyrax (chapter III). The renewed detection of MAP within the reproductive organs of rock hyraxes (chapter III) supports the hypotheses that MAP may be sexually transmitted via bull semen (chapter I). To the best of the author's knowledge, this is the second report on the presence of MAP within the reproductive organs of naturally infected non-ruminant wildlife species. Primarily, MAP was detected in the reproduction organs of rabbits (Oryctolagus cuniculus) from Scotland by PCR and bacterial culture (Judge et al., 2006). In contrast to cattle (syndesmochorial placenta), rabbits as well as rock hyraxes have a haemoendothelial placenta (Telugu and Green, 2008). In this placenta type, the uterine epithelium, the chorionic villi, and the chorionic mesenchyme are absent. Thus, embryonic capillaries are directly located in the maternal blood. Due to these anatomical characteristics, the vertical transmission of MAP in utero is much more likely in rabbits (Telugu and Green, 2008) and rock hyraxes than in cattle. Further studies are needed to investigate the sexual route of transmission in non-ruminant hosts. 
GENERAL DISCUSSION

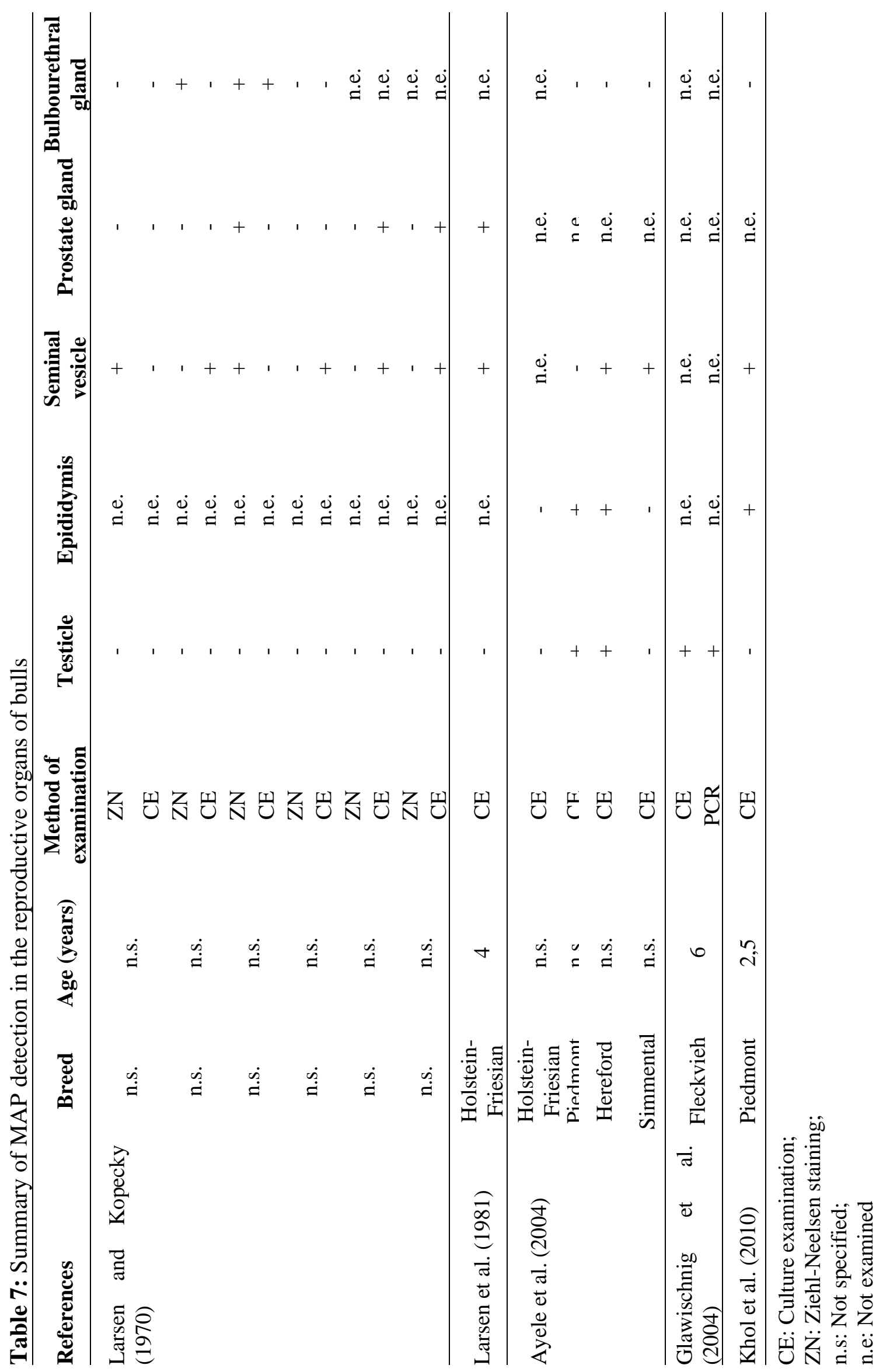


Table 8: $\quad$ Summary of MAP detection in semen.

\begin{tabular}{|c|c|c|c|c|c|c|}
\hline References & Species & $\begin{array}{r}\text { Methods of } \\
\text { examination }\end{array}$ & $\begin{array}{c}\text { Number of } \\
\text { examined } \\
\text { animals }\end{array}$ & $\begin{array}{c}\text { Number of } \\
\text { examined } \\
\text { samples }\end{array}$ & & $\begin{array}{l}\text { mber of } \\
\text { ositive } \\
\text { ples }(\%)\end{array}$ \\
\hline $\begin{array}{l}\text { Larsen and } \\
\text { Kopecky (1970) }\end{array}$ & Cattle & $\mathrm{CE}$ & 1 & 1 & 1 & $(100.0)$ \\
\hline Larsen et al. (1981) & Cattle & $\mathrm{CE}$ & 1 & 31 & 8 & $(25.8)$ \\
\hline $\begin{array}{l}\text { Eppleston and } \\
\text { Whittington (2001) }\end{array}$ & Sheep & $\mathrm{CE}$ & 11 & 11 & 3 & $(27.3)$ \\
\hline Ayele et al. (2004) & Cattle & $\mathrm{CE}$ & 1 & 100 & 1 & $(1.0)$ \\
\hline $\begin{array}{l}\text { Buergelt et al. } \\
\text { (2004) }\end{array}$ & Cattle & $\mathrm{CE}$ & 1 & 2 & 1 & $(50.0)$ \\
\hline \multirow[t]{3}{*}{ Khol et al. (2010) } & Cattle & $\mathrm{CE}$ & 1 & 9 & 0 & $(0.0 \%$ \\
\hline & & F57 qPCR & & 9 & 6 & $(66.7)$ \\
\hline & & IS900 qPCR & & 9 & 9 & $(100.0)$ \\
\hline $\begin{array}{l}\text { Sharifzadeh et al. } \\
\text { (2010) }\end{array}$ & Cattle & nPCR & 112 & 112 & 14 & $(12.5)$ \\
\hline \multirow{3}{*}{$\begin{array}{l}\text { Münster et al. } \\
\text { (2013b) }\end{array}$} & Cattle & $\mathrm{CE}$ & 1 & 40 & 0 & $(0.0)$ \\
\hline & & snPCR & & 65 & 29 & $(44,6)$ \\
\hline & & qPCR & & $29 *$ & 20 & $(69.0)$ \\
\hline $\begin{array}{l}\text { Khamesipour et al. } \\
\text { (2014) }\end{array}$ & Cattle & nPCR & 83 & 83 & 8 & $(9,6)$ \\
\hline
\end{tabular}

CE: Culture examination;

ZN: Ziehl-Neelsen staining;

n.s: Not specified;

*: Previously positive result by snPCR 


\section{Transmission of MAP between species}

Another source of infection for domestic ruminants is wild animals, which are infected with MAP and spread the pathogen into shared habitats. By the use of different multitarget genotyping methods, such as short sequence repeat (SSR) analysis, mycobacterial interspersed repetitive-unit variable-number tandem-repeat (MIRUVNTR) analysis, and restriction fragment length polymorphism analysis based on IS900 (IS900-RFLP), the transmission of MAP between wild-living red deer (Cervus elaphus) and farmed cattle herds, which shared the same habitats, has previously been demonstrated (Fritsch et al., 2012). In addition to several ruminant wildlife species, non-ruminates may also be infected with MAP. The best-studied non-ruminant MAPreservoirs are the wild rabbits of Scotland (Beard et al., 2001b; Carta et al., 2013; Greig et al., 1999). The high prevalence of MAP in rabbits, especially those in East Scotland, is associated with difficulties in controlling paratuberculosis on cattle farms (Shaughnessy et al., 2013). MAP has also been detected in many other non-ruminant species (Anderson et al., 2007; Beard et al., 2001a; Corn et al., 2005; Gronesova et al., 2008; Münster et al., 2013a); however, the number of possible infected animal species remains unclear. In this study, the occurrence of MAP in rock hyraxes (chapter III) as well as in non-human primate species (chapter II) was examined. The presence of MAP in rock hyraxes as well as in the common marmoset was demonstrated here for the first time. These descriptions expand the list of animals that seems to be susceptible to an infection with MAP. However, the impact of such infected non-ruminant species on the spread of MAP to domestic ruminants or other animals is currently unknown. Infected non-ruminant animals often do not develop clinical symptoms, and pathological and histological changes typical for paratuberculosis have been reported in only a few cases, for example in rabbits, donkeys and non-human primates (Beard et al., 2001b; McClure et al., 1987; Stief et al., 2012; Zwick et al., 2002). As demonstrated in the present investigation (chapter I), a MAP infection in asymptomatic ruminants does not always trigger clinical and/or pathological changes. For asymptomatic, infected ruminants, it is known that MAP is excreted intermittently and in varying concentrations during the subclincal stage of the disease (Mitchell et al., 2015; Münster et al., 2013b). Therefore, it can be assumed that subclinically infected non-ruminants also excrete the pathogen intermittently and in varying concentrations. Results of other studies have substantiated this hypothesis, as it has previously been demonstrated that 
subclinically infected non-ruminants can excrete pathogen concentrations of up to $10^{6}$ MAP/g faeces in rabbits (Daniels et al., 2003) and in other animal species that were kept in a German zoological garden (Münster et al., 2013b). As mentioned above (general discussion; Section: Transmission of MAP via the faeces of calves), an estimated concentration of $10^{3} \mathrm{MAP} / \mathrm{animal}$ is considered to elicit MAP infection in calves (Brotherston et al., 1961); however, the minimal infectious dose for non-ruminants is unknown. Due to the likely excretion of MAP in different concentrations, such asymptomatic non-ruminants may be sources for the unrecognized spread of MAP, on a species level and between species. For instance, in South Africa, paratuberculosis appears to be an emerging problem in sheep (Michel and Bastianello, 2000). In this area, rock hyraxes are widespread and are considered as an agricultural problem, because they compete with domestic livestock for available grazing space (Kolbe, 1967; Lensing, 1976). Due to shared habitats, it is possible that the transmission of MAP may occur between the rock hyraxes and the local livestock ruminants. Therefore, it is likely that the three wild-born rock hyraxes investigated here were naturally infected in South Africa, although it cannot completely be excluded that an infection with MAP occurred during the transport from South Africa to Germany and/or during quarantine. Further studies are required to ascertain the occurrence of MAP in the wildlife, to evaluate the potential function of the wildlife as a reservoir for MAP and the resulting impact on domestic livestock. To provide answers regarding possible routes of MAP transmission, the investigation of the MAP occurrence in local rock hyraxes, wild and domestic ruminants as well as non-ruminant species should include the analysis of MAP isolates by multitarget genotyping methods.

\section{Comparison of different antigen detection methods}

In three of the studies presented here (chapters I, II and III), MAP was more often detected by PCR than by culture examination. Analyses of the faeces, blood, semen, and tissue samples from the German Fleckvieh bull (chapter I) clearly illustrated this discrepancy. Many different factors can adversely affect the detectability of MAP. Primarily, the formation of cluster, inhomogeneous distribution and rather small numbers of MAP within the samples negatively affect the detectability of MAP by antigen detection methods. In addition to these reasons, detectability using culture approaches is negatively influenced by additional components. The required 
decontamination, which suppresses the accompanying flora, has a negative impact on the viability of MAP (Bradner et al., 2013). Furthermore, the above-mentioned ability of MAP to merge into a VBNC stage may also explain the failure of MAP growth in this investigation. The recovery rate is negatively influenced by these factors, especially in the case of rather small numbers of MAP, which are known be lower in animals without visible clinical signs than in clinically infected animals. Due to this, the sensitivity of MAP culture from the faeces of subclinically infected animals is considered to be $23-49 \%$, whereas culture of MAP from animals with clinical signs of paratuberculosis is thought to reach a sensitivity of 98\% (Nielsen and Toft, 2008). Discrepancy between the detection of MAP by PCR and culture has also been observed in many other studies (Kralik et al., 2014; Kralik et al., 2011; Münster et al., 2013b). For instance, one study investigated 1906 faecal samples of dairy cattle. In total, 875 (45.91\%) and only $169(8.87 \%)$ samples tested positive for the presence of MAP by IS900-based real-time PCR and culture, respectively (Kralik et al., 2011). Although the culture of MAP in currently considered the gold standard for diagnosis, it is necessary to remember that, especially in the case of subclinically infected animals, culture as well as PCR should be used to examine animal samples.

Methods conventionally used for the detection of MAP must be performed in wellequipped laboratories; these are ill-suited for use at the point of need. The often-used real-time PCR i) requires complex devices, ii) must be performed by highly qualified personnel and iii) the reagents must be stored at $-20{ }^{\circ} \mathrm{C}$. In contrast to real-time PCR, RPA assays are extremely fast (3-15 minutes) and all reagents are cold-chain independent. Several different RPA assays have been developed for the detection of pathogens, such as group B streptococci, Brucella or Mycobacterium tuberculosis (Boyle et al., 2014; Daher et al., 2014; Ren et al., 2016). The development and validation of an RPA assay for the detection of MAP DNA is mentioned in chapter IV of this thesis. The limit of detection was 16 DNA molecules using a molecular DNA standard, and approximately $500 \mathrm{fg}$ of the entire MAP genome. In order to enable the use of RPA assays at the point of need, a mobile suitcase laboratory was developed in our division (Figure 12) (Abd El Wahed et al., 2015). The use of the RPA assay in combination with a fast DNA extraction protocol would allow the direct detection of MAP in the field. These technologies are particularly suitable for low-resource settings or direct, on-site testing by veterinarians. However, the development of an equivalent 
fast DNA extraction protocol for the isolation of MAP is still needed. Furthermore, the combination of both methods (speed extraction and RPA) would also allow the directly analysis of environmental and faecal samples from different species in the field, as recommended in chapter III of this thesis.

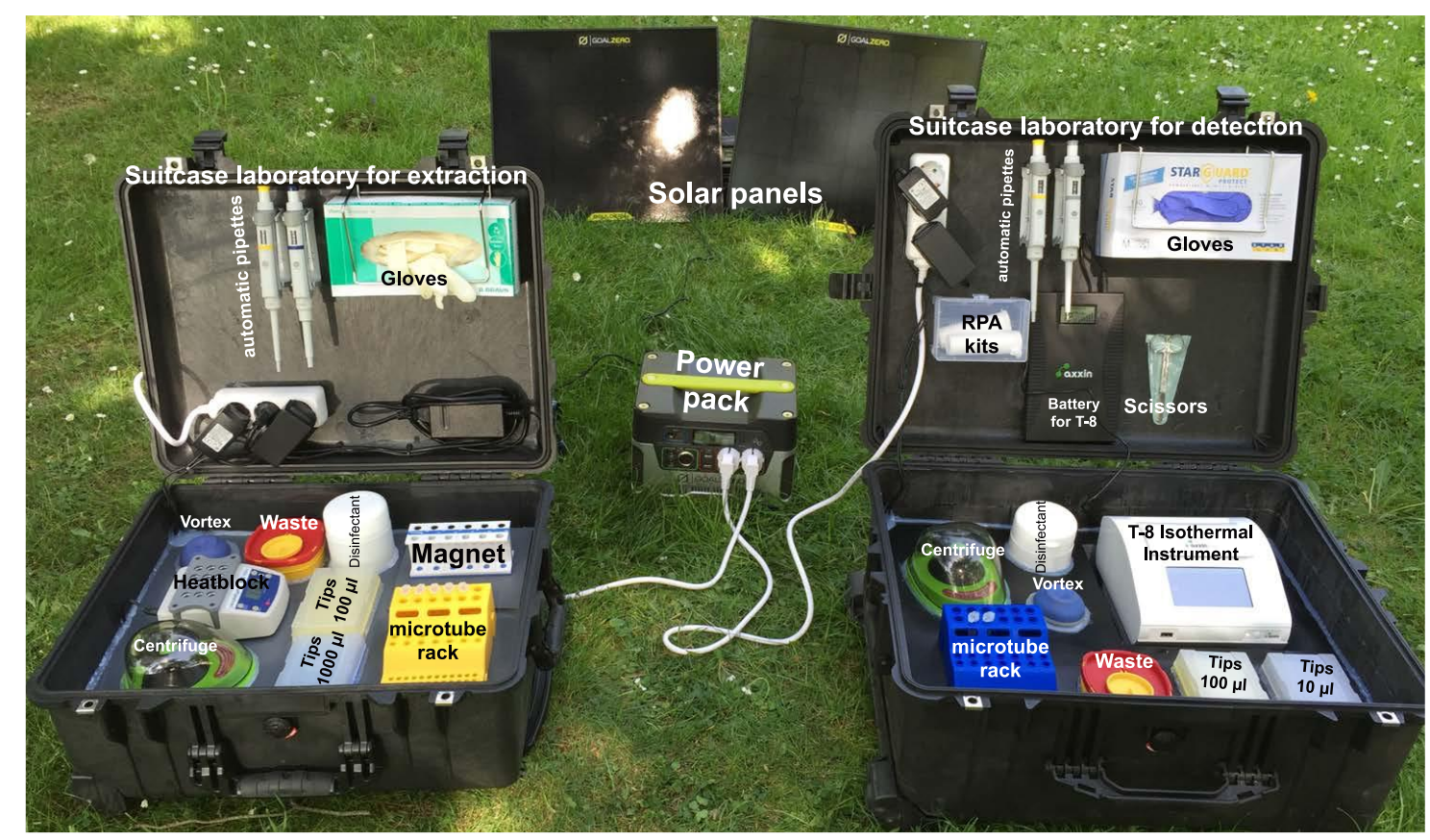

Figure 12: The mobile suitcase laboratory. The mobile setup consists of two suitcases, one for nucleic acid extraction (left suitcase) and another for detection (right suitcase), which contains all reagents and equipment for the detection of the nucleic acids of infectious agents using RPA technology. All reagents used in the mobile laboratory are cold-chain independent and powered by solar battery, making it ideal for low-resource settings (Division of Microbiology and Animal Hygiene, Georg-AugustUniversity Göttingen)

\section{Primates and Crohn's disease}

The aetiology of Crohn's disease is uncertain, and the involvement of MAP in this disease is controversial (Behr and Kapur, 2008; Feller et al., 2007). Meta-analyses have demonstrated that MAP is more often present in patients with Crohn's disease than in control patients (Abubakar et al., 2007; Feller et al., 2007); however, a causal link has not yet been demonstrated (Rudoler, 2004; Van Kruiningen, 1999). Non-human 
primates are genetically and physiologically the most similar to humans. In this investigation (chapter II), MAP DNA was detected in two different non-human primate species, again confirming the susceptibility of non-human primates to MAP infection. The presence of MAP was only demonstrated in species that belong to the Callitrichidae family. Species belonging to this family are known to often develop chronic colitis when kept in captivity (Ludlage and Mansfield, 2003), which although occurring independently is largely related to the symptom-complex of Wasting Marmoset Syndrome (WMS). The symptoms of WMS in marmosets show clear parallels to the clinical picture of Crohn's disease in humans. Due to the clinical and pathological similarities, cottontop tamarins have previously been used as an animal model for scientific studies on ulcerative colitis and Crohn's disease (Clapp et al., 1991); unfortunately, the occurrence of MAP was not monitored in these studies. Hence, non-human primates, especially marmosets, should be analysed in detail for the presence of MAP and macroscopic and microscopic pathological changes, which may be due to MAP infection. Such examinations may promote further understanding of the mechanisms involved in inflammatory bowel diseases associated with MAP infection. 


\section{CONCLUSION}

The following important conclusions were drawn in this investigation:

1. In a longitudinal study, an intermittent detection of MAP in faeces, semen and blood of a naturally infected, clinically asymptomatic German Fleckvieh bull was observed.

2. MAP was not detected on $65 \%$ of the sample days, and up to nine weeks in any matrices, demonstrating the difficulties in the confirmation of a MAP infection in asymptomatic, infected ruminants.

3. In asymptomatic, infected hosts, the presence of MAP was more frequently detected by PCR than by bacterial culture, independent of the investigated matrices.

4. Serologically negative shedders can be a source of infection for other animals.

5. A further source of infection is calves, which can excrete MAP in concentrations of up to $6.25 \times 10^{6}$ genome equivalents/g faeces.

6. The first descriptions of the detection of MAP in rock hyraxes and a common marmoset were here given. This expands the list of animals that are known to be susceptible to an infection with MAP.

7. The manifestation of MAP in most body regions in infected but asymptomatic ruminates and non-ruminate hosts was demonstrated.

8. MAP may replicate within an asymptomatic, infected host without triggering typical pathohistological changes or clinical signs of paratuberculosis.

9. It can be assumed that asymptomatic, infected non-ruminants excrete MAP intermittently and in varying concentrations, as was here demonstrated in a bull. 
10. The repeated detection of MAP in the bone marrow (Fleckvieh bull and common marmoset) may be an indication that, as described for Mycobacterium tuberculosis, viable but not culturable (VBNC) MAP cells persist in the mesenchyme cells of the bone marrow and play a key role in the establishment of a latent infection.

11. The detection of MAP in the male reproductive organs and semen of a German Fleckvieh bull supports the hypothesis that MAP may be transmitted via semen.

12. Worldwide, this is the second demonstration of the presence of MAP DNA within the reproductive organs of a naturally infected, non-ruminant species.

13. MAP seems to be a pathogen carried by free-living rock hyraxes in South Africa, and may be a potential source of infection for local livestock animals.

14. A sensitive (16 DNA molecules) and rapid (15 min) RPA assay was developed to allow the detection of MAP in poor settings or directly at the point of need.

15. Confirmation that non-human primates, which are genetically and physiologically similar to humans, are susceptible to MAP infection. 


\section{FUTURE PROSPECTS}

The further prospects for this work are:

1. Development of new strategies for the reliable diagnosis of subclinical paratuberculosis.

2. Investigation of the occurrence of MAP in the bone marrow of infected animals, in addition to the gastrointestinal tissues and lymph nodes, to substantiate the hypothesis that the bone marrow may provide a niche for the establishment of a latent MAP infection.

3. Development of a sensitive and specific method to detect viable MAP in semen, to monitor the occurrence of MAP in the semen of subclinical as well as clinically infected breeding bulls.

4. Test the influence of antibiotics in the various semen extenders, as well as the effect of the freezing process on the viability of MAP in semen.

5. Analysis of further animal species with a haemoendothelial placenta to investigate this route of transmission.

6. Monitoring of the occurrence of MAP in different wildlife species to identify further potential reservoirs.

7. Development of a speed extraction protocol of MAP in faeces, with the aim of implementing the RPA assay in this field.

8. Detailed analysis of further MAP-infected non-human primates to understand the mechanisms of inflammatory bowel disease. 
REFERENCES

\section{REFERENCES}

Abbas, M., Munir, M., Khaliq, S.A., Haq, M.I., Tanveer Khan, M., Qureshi, Z.U., 2011. Detection of paratuberculosis in breeding bulls at pakistani semen production units: a continuous source of threat. ISRN veterinary science 2011, 501235.

Abd El Wahed, A., El-Deeb, A., El-Tholoth, M., Abd El Kader, H., Ahmed, A., Hassan, S., Hoffmann, B., Haas, B., Shalaby, M.A., Hufert, F.T., Weidmann, M., $2013 a$. A portable reverse transcription recombinase polymerase amplification assay for rapid detection of foot-and-mouth disease virus. PloS one 8, e71642.

Abd El Wahed, A., Patel, P., Heidenreich, D., Hufert, F.T., Weidmann, M., 2013b. Reverse transcription recombinase polymerase amplification assay for the detection of middle East respiratory syndrome coronavirus. PLoS currents 5.

Abd El Wahed, A., Weidmann, M., Hufert, F.T., 2015. Diagnostics-in-a-Suitcase: Development of a portable and rapid assay for the detection of the emerging avian influenza A (H7N9) virus. Journal of Clinical Virology 69, 16-21.

Abubakar, I., Myhill, D.J., Hart, A.R., Lake, I.R., Harvey, I., Rhodes, J.M., Robinson, R., Lobo, A.J., Probert, C.S., Hunter, P.R., 2007. A case-control study of drinking water and dairy products in Crohn's Disease-further investigation of the possible role of Mycobacterium avium paratuberculosis. American journal of epidemiology 165, 776-783.

Alexejeff-Goleff, N.A., 1929. Question on pathogenesis and pathogen excretion in cattle paratuberculosis [Zur Frage der Pathogenese und Bazillenausscheidung bei Rinderparatuberkulose]. Z. Infekt. Krank. Haus. 36, 312-317.

Alonso-Hearn, M., Molina, E., Geijo, M., Vazquez, P., Sevilla, I., Garrido, J.M., Juste, R.A., 2009. Isolation of Mycobacterium avium subsp. paratuberculosis from muscle tissue of naturally infected cattle. Foodborne pathogens and disease 6, 513-518.

Amer, H.M., Abd El Wahed, A., Shalaby, M.A., Almajhdi, F.N., Hufert, F.T., Weidmann, M., 2013. A new approach for diagnosis of bovine coronavirus using a reverse transcription recombinase polymerase amplification assay. Journal of virological methods 193, 337-340.

Anderson, J.L., Meece, J.K., Koziczkowski, J.J., Clark, D.L., Jr., Radcliff, R.P., Nolden, C.A., Samuel, M.D., Ellingson, J.L., 2007. Mycobacterium avium subsp. 
paratuberculosis in scavenging mammals in Wisconsin. Journal of wildlife diseases 43, 302-308.

Anonymous, 2005. Guidelines for the management of paratuberculosis in ruminants [Leitlinien für den Umgang mit Paratuberkulose in Wiederkäuerbeständen]. Bundesministerium für Verbraucherschutz, Ernährung und Landwirtschaft.

Anonymous, 2011. Ordinance on Notifiable Animal Diseases [Verordnung über meldepflichtige Tierkrankheiten]. Bundesgesetzblatt Jahrgang 2011 Teil I Nr. 7, 252-253.

Anonymous, 2015. Program for the control of paratuberculosis in cattle herds in Thuringia [Programm zur Bekämpfung der Paratuberkulose in den Rinderbeständen in Thüringen]. Ministry of Labor, Social Affairs, Health, Women and Families; Amtliche Mitteilung 26/2015.

Antognoli, M.C., Garry, F.B., Hirst, H.L., Lombard, J.E., Dennis, M.M., Gould, D.H., Salman, M.D., 2008. Characterization of Mycobacterium avium subspecies paratuberculosis disseminated infection in dairy cattle and its association with antemortem test results. Veterinary microbiology 127, 300-308.

Ayele, W.Y., Bartos, M., Svastova, P., Pavlik, I., 2004. Distribution of Mycobacterium avium subsp. paratuberculosis in organs of naturally infected bull-calves and breeding bulls. Veterinary microbiology 103, 209-217.

Ayele, W.Y., Svastova, P., Roubal, P., Bartos, M., Pavlik, I., 2005. Mycobacterium avium subspecies paratuberculosis cultured from locally and commercially pasteurized cow's milk in the Czech Republic. Applied and environmental microbiology $71,1210-1214$.

Bakker, D., 2010. Paratuberculosis Control Measures in Europe. In: Paratuberculosis: Organism, Disease, Control. Behr, M. A., \& Collins, D. M. (Eds.), CABI., 306318.

Balseiro, A., Marin, J.F.G., Solano, P., Garrido, J.M., Prieto, J.M., 2008. Histopathological classification of lesions observed in natural cases of paratuberculosis in free-ranging fallow deer (Dama dama). Journal of comparative pathology 138, 180-188.

Bang, B., 1906. Chronic pseudotuberculous inflammation in cattle [Chronische pseudotuberkulöse Darmentzündung beim Rinde]. Berliner und Münchener tierärztliche Wochenschrift 42, 759-763. 
Bang, D., Herlin, T., Stegger, M., Andersen, A.B., Torkko, P., Tortoli, E., Thomsen, V.O., 2008. Mycobacterium arosiense sp. nov., a slowly growing, scotochromogenic species causing osteomyelitis in an immunocompromised child. International journal of systematic and evolutionary microbiology 58, 2398-2402.

Bätza, H.-J., 2014. Announcement of recommendations for hygienic requirements for the retention of ruminants [Bekanntmachung von Empfehlungen für hygienische Anforderungen an das Halten von Wiederkäuern]. Federal Ministry of Food and Agriculture; BAnz. AT 01.08.2014 B1.

Beard, P., Henderson, D., Daniels, M., Pirie, A., Buxton, D., Greig, A., Hutchings, M., McKendrick, I., Rhind, S., Stevenson, K., 1999. Evidence of paratuberculosis in fox. Veterinary Record 145, 612-613.

Beard, P.M., Daniels, M.J., Henderson, D., Pirie, A., Rudge, K., Buxton, D., Rhind, S., Greig, A., Hutchings, M.R., McKendrick, I., Stevenson, K., Sharp, J.M., 2001a. Paratuberculosis infection of nonruminant wildlife in Scotland. Journal of clinical microbiology 39, 1517-1521.

Beard, P.M., Rhind, S.M., Buxton, D., Daniels, M.J., Henderson, D., Pirie, A., Rudge, K., Greig, A., Hutchings, M.R., Stevenson, K., Sharp, J.M., 2001b. Natural paratuberculosis infection in rabbits in Scotland. Journal of comparative pathology 124, 290-299.

Begg, D.J., Whittington, R.J., 2008. Experimental animal infection models for Johne's disease, an infectious enteropathy caused by Mycobacterium avium subsp. paratuberculosis. Veterinary journal 176, 129-145.

Behr, M.A., Kapur, V., 2008. The evidence for Mycobacterium paratuberculosis in Crohn's disease. Current opinion in gastroenterology 24, 17-21.

Ben Salah, I., Cayrou, C., Raoult, D., Drancourt, M., 2009. Mycobacterium marseillense sp. nov., Mycobacterium timonense sp. nov. and Mycobacterium bouchedurhonense sp. nov., members of the Mycobacterium avium complex. International journal of systematic and evolutionary microbiology 59, 28032808.

Benazzi, S., Hamidi, M., Schliesser, T., 1996. Paratuberculosis in sheep flocks in Morocco: a serological, microscopical and cultural survey. Journal of Veterinary Medicine, Series B 43, 213-219. 
Berg, J.J., Jensen, P., 1978. Enzyme-linked immunosorbent assay (ELISA) for detection of antibodies to Mycobacterium paratuberculosis in cattle. Acta Veterinaria Scandinavica 19, 310.

Boelaert, F., Walravens, K., Biront, P., Vermeersch, J., Berkvens, D., Godfroid, J., 2000. Prevalence of paratuberculosis (Johne's disease) in the Belgian cattle population. Veterinary microbiology 77, 269-281.

Botsaris, G., Swift, B.M., Slana, I., Liapi, M., Christodoulou, M., Hatzitofi, M., Christodoulou, V., Rees, C.E., 2016. Detection of viable Mycobacterium avium subspecies paratuberculosis in powdered infant formula by phage-PCR and confirmed by culture. International journal of food microbiology 216, 91-94.

Boyle, D.S., McNerney, R., Teng Low, H., Leader, B.T., Perez-Osorio, A.C., Meyer, J.C., O'Sullivan, D.M., Brooks, D.G., Piepenburg, O., Forrest, M.S., 2014. Rapid detection of Mycobacterium tuberculosis by recombinase polymerase amplification. PloS one 9, e103091.

Bradner, L., Robbe-Austerman, S., Beitz, D.C., Stabel, J.R., 2013. Optimization of hexadecylpyridinium chloride decontamination for culture of Mycobacterium avium subsp. paratuberculosis from milk. Journal of clinical microbiology 51, 1575-1577.

Brady, C., O'Grady, D., O'Meara, F., Egan, J., Bassett, H., 2008. Relationships between clinical signs, pathological changes and tissue distribution of Mycobacterium avium subspecies paratuberculosis in 21 cows from herds affected by Johne's disease. The Veterinary record 162, 147-152.

Brotherston, J.G., Gilmour, N., Samuel, J.M., 1961. Quantitative studies of Mycobacterium johnei in the tissues of sheep: I. Routes of infection and assay of viable M. johnei. Journal of Comparative Pathology and Therapeutics 71, 286299.

Buergelt, C. 1991. Aspects of the pathology of paratuberculosis. In: Proceedings of the 3th International Colloquium on Paratuberculosis, Orlando (USA), Sept 28 - Oct 2, 136-139.

Buergelt, C.D., Donovan, G.A., Williams, J.E., 2004. Identification of Mycobacterium avium subspecies paratuberculosis by polymerase chain reaction in blood and semen of a bull with clinical paratuberculosis. The International Journal of Applied Research in Veterinary Medicine 2, 130-134. 
Buergelt, C.D., Hall, C., McEntee, K., Duncan, J.R., 1978. Pathological evaluation of paratuberculosis in naturally infected cattle. Veterinary pathology 15, 196-207.

Buergelt, C.D., Williams, J.E., 2004. Nested PCR on blood and milk for the detection of Mycobacterium avium subsp paratuberculosis DNA in clinical and subclinical bovine paratuberculosis. Australian veterinary journal 82, 497-503.

Büttner, M., Gerbermann, H., Naumann, L., Neuendorf, E., Rinder, H., Wildner, M., Zapf, A., 2006. Paratuberculosis in cattle-Crohn's disease in humans: a causal link [Paratuberkulose beim Rind-Morbus Crohn beim Menschen: ein ursächlicher Zusammenhang]. Bayerisches Landesamt für Gesundheit und Lebensmittelsicherheit 1-114.

Carta, T., Alvarez, J., Perez de la Lastra, J.M., Gortazar, C., 2013. Wildlife and paratuberculosis: a review. Research in veterinary science 94, 191-197.

Cetinkaya, B., Erdogan, H.M., Morgan, K.L., 1997. Relationships between the presence of Johne's disease and farm and management factors in dairy cattle in England. Preventive veterinary medicine 32, 253-266.

Challans, J., Stevenson, K., Reid, H., Sharp, J., 1994. A rapid method for the extraction and detection of Mycobacterium avium subspecies paratuberculosis from clinical specimens. Veterinary Record 134, 95-96.

Chiodini, R.J., 1996. Immunology: resistance to paratuberculosis. The Veterinary clinics of North America. Food animal practice 12, 313-343.

Chiodini, R.J. 2005. The History of Paratuberculosis. In: Proceedings of the 8th International Colloquium on Paratuberculosis, Copenhagen (Denmark), August 14-17, 2005, 658-660.

Chiodini, R.J., Rossiter, C.A., 1996. Paratuberculosis: a potential zoonosis? The Veterinary clinics of North America. Food animal practice 12, 457-467.

Chiodini, R.J., Van Kruiningen, H.J., 1983. Eastern white-tailed deer as a reservoir of ruminant paratuberculosis. Journal of the American Veterinary Medical Association 182, 168-169.

Chiodini, R.J., Van Kruiningen, H.J., Merkal, R.S., 1984a. Ruminant paratuberculosis (Johne's disease): the current status and future prospects. The Cornell veterinarian $74,218-262$.

Chiodini, R.J., Van Kruiningen, H.J., Thayer, W.R., Merkal, R.S., Coutu, J.A., 1984b. Possible role of mycobacteria in inflammatory bowel disease. I. An unclassified 
Mycobacterium species isolated from patients with Crohn's disease. Digestive diseases and sciences 29, 1073-1079.

Clapp, N.K., Henke, M.A., Hansard, R.M., Walsh, R.E., Widomski, D.L., Anglin, C.P., Fretland, D.J., Gaginella, T.S., 1991. Inflammatory mediators in cotton-top tamarins (CTT) with acute and chronic colitis. Agents and actions 34, 178-180.

Clarke, C.J., 1997. The pathology and pathogenesis of paratuberculosis in ruminants and other species. Journal of comparative pathology 116, 217-261.

Collins, D.M., Gabric, D.M., de Lisle, G.W., 1990. Identification of two groups of Mycobacterium paratuberculosis strains by restriction endonuclease analysis and DNA hybridization. Journal of clinical microbiology 28, 1591-1596.

Collins, M.T., 1996. Diagnosis of paratuberculosis. Veterinary Clinics of North America: Food Animal Practice 12, 357-371.

Collins, M.T., 1997. Mycobacterium paratuberculosis: a potential food-borne pathogen? Journal of dairy science $80,3445-3448$.

Collins, M.T., Zhao, B.Y. 1994. Comparison of the commercial serum antibody ELISA, -interferon test kit, and radiometric fecal culture for early diagnosis of paratuberculosis in experimentally infected Holstein calves. In: Proceedings of the Fourth International Colloquium on Paratuberculosis., Cambridge (United Kingdom), July 17-21, 1994, 67-76.

Condron, R.J., Schroen, C.J., Black, C.A., Ridge, S.E., Hope, A.F. 1994. Histological confirmation of subclinical infection with M. paratuberculosis in cattle. In: Proceedings of the Fourth International Colloquium on Paratuberculosis, Cambridge (United Kingdom), July 17-21, 1994, 37-40.

Cook, J., Xu, W., Msellem, M., Vonk, M., Bergstrom, B., Gosling, R., Al-Mafazy, A.W., McElroy, P., Molteni, F., Abass, A.K., Garimo, I., Ramsan, M., Ali, A., Martensson, A., Bjorkman, A., 2015. Mass screening and treatment on the basis of results of a Plasmodium falciparum-specific rapid diagnostic test did not reduce malaria incidence in Zanzibar. The Journal of infectious diseases 211, 1476-1483.

Corn, J.L., Manning, E.J., Sreevatsan, S., Fischer, J.R., 2005. Isolation of Mycobacterium avium subsp. paratuberculosis from free-ranging birds and mammals on livestock premises. Applied and environmental microbiology 71, 6963-6967. 
Daffe, M., Draper, P., 1998. The envelope layers of mycobacteria with reference to their pathogenicity. Advances in microbial physiology 39, 131-203.

Daher, R.K., Stewart, G., Boissinot, M., Bergeron, M.G., 2014. Isothermal recombinase polymerase amplification assay applied to the detection of group B streptococci in vaginal/anal samples. Clinical Chemistry 60, 660-666.

Dalziel, T.K., 1913. Chronic intestinal enteritis. . British Medical Journal 2, 1068-1070.

Daniels, M., Henderson, D., Greig, A., Stevenson, K., Sharp, J., Hutchings, M., 2003. The potential role of wild rabbits Oryctolagus cuniculus in the epidemiology of paratuberculosis in domestic ruminants. Epidemiology and infection 130, 553.

Dargatz, D.A., Byrum, B.A., Barber, L.K., Sweeney, R.W., Whitlock, R.H., Shulaw, W.P., Jacobson, R.H., Stabel, J.R., 2001. Evaluation of a commercial ELISA for diagnosis of paratuberculosis in cattle. Journal of the American Veterinary Medical Association 218, 1163-1166.

Das, B., Kashino, S.S., Pulu, I., Kalita, D., Swami, V., Yeger, H., Felsher, D.W., Campos-Neto, A., 2013. CD271(+) bone marrow mesenchymal stem cells may provide a niche for dormant Mycobacterium tuberculosis. Science translational medicine 5, 170ra113.

Deutz, A., Spergser, J., Wagner, P., Rosengarten, R., Kofer, J., 2005. Mycobacterium avium subsp. paratuberkulose bei Wildtierarten und Rindern in der Steiermark / Österreich [Mycobacterium avium subsp. paratuberculosis in wild animal species and cattle in Styria/Austria]. Berliner und Münchener tierärztliche Wochenschrift 118, 314-320.

Dirksen, G., Gründer, H.-D., Stöber, M., 2003. Internal medicine and surgery of cattle [Innere Medizin und Chirurgie des Rindes], Vol 4. Parey Buchverlag, Berlin.

Doyle, T.M., 1954. Isolation of Johne's bacilli from the udders of clinically affected cows. British Veterinary Journal 110, 215-218.

Doyle, T.M., 1958. Foetal infection in Johne's disease. Veterinary Record 70, 328.

Eamens, G., Whittington, R., Marsh, I., Turner, M., Saunders, V., Kemsley, P., Rayward, D., 2000. Comparative sensitivity of various faecal culture methods and ELISA in dairy cattle herds with endemic Johne's disease. Veterinary microbiology 77, 357-367.

Eisenberg, S.W., Koets, A.P., Hoeboer, J., Bouman, M., Heederik, D., Nielen, M., 2010a. Presence of Mycobacterium avium subsp. paratuberculosis in 
environmental samples collected on commercial Dutch dairy farms. Applied and environmental microbiology 76, 6310-6312.

Eisenberg, S.W., Nielen, M., Santema, W., Houwers, D.J., Heederik, D., Koets, A.P., 2010b. Detection of spatial and temporal spread of Mycobacterium avium subsp. paratuberculosis in the environment of a cattle farm through bio-aerosols. Veterinary microbiology 143, 284-292.

Ellingson, J.L., Anderson, J.L., Koziczkowski, J.J., Radcliff, R.P., Sloan, S.J., Allen, S.E., Sullivan, N.M., 2005. Detection of viable Mycobacterium avium subsp. paratuberculosis in retail pasteurized whole milk by two culture methods and PCR. Journal of food protection 68, 966-972.

Ellingson, J.L., Bolin, C.A., Stabel, J.R., 1998. Identification of a gene unique to Mycobacterium avium subspecies paratuberculosis and application to diagnosis of paratuberculosis. Molecular and cellular probes 12, 133-142.

Englund, S., Bölske, G., Ballagi-Pordany, A., Johansson, K.-E., 2001. Detection of Mycobacterium avium subsp. paratuberculosis in tissue samples by single, fluorescent and nested PCR based on the IS900 gene. Veterinary microbiology $81,257-271$.

Engvall, E., Perlmann, P., 1971. Enzyme-linked immunosorbent assay (ELISA) quantitative assay of immunoglobulin G. Immunochemistry 8, 871-874.

Eppleston, J., Whittington, R.J., 2001. Isolation of Mycobacterium avium subsp. paratuberculosis from the semen of rams with clinical Johne's disease. Australian veterinary journal 79, 776-777.

Euler, M., Wang, Y., Nentwich, O., Piepenburg, O., Hufert, F.T., Weidmann, M., 2012a. Recombinase polymerase amplification assay for rapid detection of Rift Valley fever virus. Journal of Clinical Virology 54, 308-312.

Euler, M., Wang, Y., Otto, P., Tomaso, H., Escudero, R., Anda, P., Hufert, F.T., Weidmann, M., 2012b. Recombinase Polymerase Amplification Assay for Rapid Detection of Francisella tularensis. Journal of clinical microbiology 50, 2234-2238.

Fecteau, M.E., Whitlock, R.H., Buergelt, C.D., Sweeney, R.W., 2010. Exposure of young dairy cattle to Mycobacterium avium subsp. paratuberculosis (MAP) through intensive grazing of contaminated pastures in a herd positive for Johne's disease. The Canadian veterinary journal. 51, 198-200. 
Feller, M., Huwiler, K., Stephan, R., Altpeter, E., Shang, A., Furrer, H., Pfyffer, G.E., Jemmi, T., Baumgartner, A., Egger, M., 2007. Mycobacterium avium subspecies paratuberculosis and Crohn's disease: a systematic review and meta-analysis. The Lancet. Infectious diseases 7, 607-613.

Ferreira, R., Fonseca, L., Lilenbaum, W., 2002. Comparison between a commercial and an in-house ELISA for anti-M. avium paratuberculosis antibodies detection in dairy herds in Rio de Janeiro, Brazil. Revista latinoamericana de microbiologia 44, 129-132.

Foddai, A., Strain, S., Whitlock, R.H., Elliott, C.T., Grant, I.R., 2011. Application of a peptide-mediated magnetic separation-phage assay for detection of viable Mycobacterium avium subsp. paratuberculosis to bovine bulk tank milk and feces samples. Journal of clinical microbiology 49, 2017-2019.

Foddai, A.C., Grant, I.R., 2017. Sensitive and specific detection of viable Mycobacterium avium subsp. paratuberculosis in raw milk by the peptidemediated magnetic separation-phage assay. Journal of applied microbiology $122,1357-1367$.

Fritsch, I., Luyven, G., Köhler, H., Lutz, W., Möbius, P., 2012. Suspicion of Mycobacterium avium subsp. paratuberculosis transmission between cattle and wild-living red deer (Cervus elaphus) by multitarget genotyping. Applied and environmental microbiology 78, 1132-1139.

Gasteiner, J., Wenzl, H., Fuchs, K., Jark, U., Baumgartner, W., 1999. Serological Cross-sectional Study of Paratuberculosis in Cattle in Austria. Zoonoses and public health 46, 457-466.

Gay, J.M., Sherman, D.M., 1992. Factors in the Epidemiology and Control of Ruminant Paratuberculosis. Veterinary medicine (USA) 87, 1133-1139.

Gerlach, G.F., 2002. Paratuberculosis: the pathogen and routes of infection [Paratuberkulose: der Erreger und Infektionswege]. Deutsche tierärztliche Wochenschrift 109, 504-506.

Ghadiali, A.H., Strother, M., Naser, S.A., Manning, E.J., Sreevatsan, S., 2004. Mycobacterium avium subsp. paratuberculosis strains isolated from Crohn's disease patients and animal species exhibit similar polymorphic locus patterns. Journal of clinical microbiology 42, 5345-5348.

Glawischnig, W., Awad-Masalmeh, M., Khaschabi, D., Schonbauer, M., 2004. Detection of Mycobacterium avium subsp. paratuberculosis from the testicles of 
a clinically infected breeding animal [Nachweis von Mycobacterium avium subsp. paratuberkulose aus den Hoden eines klinisch infizierten Zuchttiers]. Berliner und Münchener tierärztliche Wochenschrift 117, 136-139.

Godfroid, J., Delcorps, C., Irenge, L.M., Walravens, K., Marche, S., Gala, J.L., 2005. Definitive differentiation between single and mixed mycobacterial infections in red deer (Cervus elaphus) by a combination of duplex amplification of p34 and f57 sequences and Hpy188I enzymatic restriction of duplex amplicons. Journal of clinical microbiology 43, 4640-4648.

Gonda, M.G., Chang, Y.M., Shook, G.E., Collins, M.T., Kirkpatrick, B.W., 2006. Genetic variation of Mycobacterium avium ssp. paratuberculosis infection in US Holsteins. Journal of dairy science 89, 1804-1812.

Grant, I.R., Ball, H.J., Rowe, M.T., 1998. Effect of high-temperature, short-time (HTST) pasteurization on milk containing low numbers of Mycobacterium paratuberculosis. Letters in applied microbiology 26, 166-170.

Grant, I.R., Ball, H.J., Rowe, M.T., 2002. Incidence of Mycobacterium paratuberculosis in bulk raw and commercially pasteurized cows' milk from approved dairy processing establishments in the United Kingdom. Applied and environmental microbiology 68, 2428-2435.

Grant, I.R., Rowe, M.T., Dundee, L., Hitchings, E., 2001. Mycobacterium avium ssp. paratuberculosis: its incidence, heat resistance and detection in milk and dairy products. International Journal of Dairy Technology 54, 2-13.

Green, E.P., Tizard, M.L., Moss, M.T., Thompson, J., Winterbourne, D.J., McFadden, J.J., Hermon-Taylor, J., 1989. Sequence and characteristics of IS900, an insertion element identified in a human Crohn's disease isolate of Mycobacterium paratuberculosis. Nucleic acids research 17, 9063-9073.

Greig, A., Stevenson, K., Henderson, D., Perez, V., Hughes, V., Pavlik, I., Hines, M.E., 2nd, McKendrick, I., Sharp, J.M., 1999. Epidemiological study of paratuberculosis in wild rabbits in Scotland. Journal of clinical microbiology 37, 1746-1751.

Greig, A., Stevenson, K., Perez, V., Pirie, A.A., Grant, J.M., Sharp, J.M., 1997. Paratuberculosis in wild rabbits (Oryctolagus cuniculus). The Veterinary record 140, 141-143.

Gronesova, P., Ficova, M., Mizakova, A., Kabat, P., Trnka, A., Betakova, T., 2008. Prevalence of avian influenza viruses, Borrelia garinii, Mycobacterium avium, 
and Mycobacterium avium subsp. paratuberculosis in waterfowl and terrestrial birds in Slovakia, 2006. Avian pathology 37, 537-543.

Gumber, S., Eamens, G., Whittington, R., 2006. Evaluation of a Pourquier ELISA kit in relation to agar gel immunodiffusion (AGID) test for assessment of the humoral immune response in sheep and goats with and without Mycobacterium paratuberculosis infection. Veterinary microbiology 115, 91-101.

Gusev, Y., Sparkowski, J., Raghunathan, A., Ferguson, H., Jr., Montano, J., Bogdan, N., Schweitzer, B., Wiltshire, S., Kingsmore, S.F., Maltzman, W., Wheeler, V., 2001. Rolling circle amplification: a new approach to increase sensitivity for immunohistochemistry and flow cytometry. The American journal of pathology 159, 63-69.

Gwozdz, J.M., 2006. Comparative evaluation of two decontamination methods for the isolation of Mycobacterium avium subspecies paratuberculosis from faecal slurry and sewage. Veterinary microbiology 115, 358-363.

Harris, N.B., Barletta, R.G., 2001. Mycobacterium avium subsp. paratuberculosis in Veterinary Medicine. Clinical microbiology reviews 14, 489-512.

Hasonova, L., Trcka, I., Babak, V., Rozsypalova, Z., Pribylova, R., Pavlik, I., 2009. Distribution of Mycobacterium avium subsp. paratuberculosis in tissues of naturally infected cattle as affected by age. Veterinarni Medicina 54, 257-269.

Hinger, M., Brandt, H., Erhardt, G., 2008. Heritability estimates for antibody response to Mycobacterium avium subspecies paratuberculosis in German Holstein cattle. Journal of dairy science $91,3237-3244$.

Hopkins, H., Gonzalez, I.J., Polley, S.D., Angutoko, P., Ategeka, J., Asiimwe, C., Agaba, B., Kyabayinze, D.J., Sutherland, C.J., Perkins, M.D., Bell, D., 2013. Highly sensitive detection of malaria parasitemia in a malaria-endemic setting: performance of a new loop-mediated isothermal amplification kit in a remote clinic in Uganda. The Journal of infectious diseases 208, 645-652.

Hughes, V., Stevenson, K., Sharp, J., 2001. Improved preparation of high molecular weight DNA for pulsed-field gel electrophoresis from mycobacteria. Journal of microbiological methods 44, 209-215.

Hutchings, M.R., Stevenson, K., Greig, A., Davidson, R.S., Marion, G., Judge, J., 2010. Infection of non-ruminant wildlife by Mycobacterium avium subsp. paratuberculosis. In: Paratuberculosis: Organism, Disease, Control. Behr, M. A., \& Collins, D. M. (Eds.), CABI., 188-200. 
Jakobsen, M.B., Alban, L., Nielsen, S.S., 2000. A cross-sectional study of paratuberculosis in 1155 Danish dairy cows. Preventive veterinary medicine 46, $15-27$.

Johne, H.A., Fortingham, L., 1895. A peculiar case of tuberculosis in cattle [Ein eigentümlicher Fall von Tuberkulose beim Rind]. Deutsche Zeitschrift für Tiermedizin und vergleichende Pathologie 21, 438-454.

Jorgensen, J.B., 1977. Survival of Mycobacterium paratuberculosis in slurry. Nordisk veterinaermedicin $29,267-270$.

Judge, J., Kyriazakis, I., Greig, A., Davidson, R.S., Hutchings, M.R., 2006. Routes of intraspecies transmission of Mycobacterium avium subsp. paratuberculosis in rabbits (Oryctolagus cuniculus): a field study. Applied and environmental microbiology 72, 398-403.

Juste, R.A., Garrido, J.M., Geijo, M., Elguezabal, N., Aduriz, G., Atxaerandio, R., Sevilla, I., 2005. Comparison of blood polymerase chain reaction and enzymelinked immunosorbent assay for detection of Mycobacterium avium subsp. paratuberculosis infection in cattle and sheep. Journal of veterinary diagnostic investigation $17,354-359$.

Kennedy, D., Benedictus, G., 2001. Control of Mycobacterium avium subsp. paratuberculosis infection in agricultural species. Revue Scientifique et Technique-Office International des Epizooties 20, 151-179.

Khamesipour, F., Doosti, A., Mazrouei Sebdani, M., 2014. Survey for the Presence of Mycobacterium avium subsp. paratuberculosis in the Bull Frozen Semen Samples and Blood Samples of Cattle, Sheep and Camel by Nested-PCR. Kafkas Universitesi Veteriner Fakultesi Dergisi 20, 681-686.

Khol, J.L., Kralik, P., Slana, I., Beran, V., Aurich, C., Baumgartner, W., Pavlik, I., 2010. Consecutive excretion of Mycobacterium avium subspecies paratuberculosis in semen of a breeding bull compared to the distribution in feces, tissue and blood by IS900 and F57 quantitative real-time PCR and culture examinations. The Journal of veterinary medical science 72, 1283-1288.

Kim, B.J., Math, R.K., Jeon, C.O., Yu, H.K., Park, Y.G., Kook, Y.H., Kim, B.J., 2013. Mycobacterium yongonense sp. nov., a slow-growing non-chromogenic species closely related to Mycobacterium intracellulare. International journal of systematic and evolutionary microbiology 63, 192-199. 
Kirkeby, C., Graesboll, K., Nielsen, S.S., Christiansen, L.E., Toft, N., Rattenborg, E., Halasa, T., 2016. Simulating the Epidemiological and Economic Impact of Paratuberculosis Control Actions in Dairy Cattle. Frontiers in veterinary science 3,90 .

Kirkwood, C.D., Wagner, J., Boniface, K., Vaughan, J., Michalski, W.P., Catto-Smith, A.G., Cameron, D.J., Bishop, R.F., 2009. Mycobacterium avium subspecies paratuberculosis in children with early-onset Crohn's disease. Inflammatory bowel diseases 15, 1643-1655.

Koenig, G., Hoffsis, G., Shulaw, W., Bech-Nielsen, S., Rings, D., St-Jean, G., 1993. Isolation of Mycobacterium paratuberculosis from mononuclear cells in tissues, blood, and mammary glands of cows with advanced paratuberculosis. American journal of veterinary research 54, 1441-1445.

Koets, A.P., Adugna, G., Janss, L.L., van Weering, H.J., Kalis, C.H., Wentink, G.H., Rutten, V.P., Schukken, Y.H., 2000. Genetic variation of susceptibility to Mycobacterium avium subsp. paratuberculosis infection in dairy cattle. Journal of dairy science $83,2702-2708$.

Köhler, H., 2005. Epidemiology, diagnosis and control of paratuberculosis [Epidemiologie, Diagnostik und Bekampfung der Paratuberkulose]. Tierärztliche Umschau 60, 492.

Köhler, H., Burkert, B., 2003. Validation of ELISAs for detection of antibodies against Mycobacterium avium ssp. paratuberculosis in cattle [Validierung von ELISAs zum Nachweis von Antikörpern gegen Mycobacterium avium ssp. paratuberculosis bei Rindern]. IVD-Infol der BFAV Insel Riems 3, 6-9.

Köhler, H., Burkert, B., Pavlik, I., Diller, R., Geue, L., Conraths, F.J., Martin, G., 2007. Evaluation of five ELISA test kits for the measurement of antibodies against Mycobacterium avium subspecies paratuberculosis in bovine serum. Berliner und Münchener tierärztliche Wochenschrift 121, 203-210.

Kolbe, F.F., 1967. A plea for dassies. African Wildlife 21, 237-248.

Kralik, P., Pribylova-Dziedzinska, R., Kralova, A., Kovarcik, K., Slana, I., 2014. Evidence of passive faecal shedding of Mycobacterium avium subsp. paratuberculosis in a Limousin cattle herd. Veterinary journal 201, 91-94.

Kralik, P., Slana, I., Kralova, A., Babak, V., Whitlock, R.H., Pavlik, I., 2011. Development of a predictive model for detection of Mycobacterium avium 
subsp. paratuberculosis in faeces by quantitative real time PCR. Veterinary microbiology 149, 133-138.

Lambrecht, R.S., Carriere, J.F., Collins, M.T., 1988. A model for analyzing growth kinetics of a slowly growing Mycobacterium sp. Applied and environmental microbiology 54, 910-916.

Lamont, E.A., Bannantine, J.P., Armien, A., Ariyakumar, D.S., Sreevatsan, S., 2012. Identification and characterization of a spore-like morphotype in chronically starved Mycobacterium avium subsp. paratuberculosis cultures. PloS one 7, e30648.

Larsen, A., Merkal, R., Cutlip, R., 1975. Age of cattle as related to resistance to infection with Mycobacterium paratuberculosis. American journal of veterinary research 36, 255-257.

Larsen, A.B., Kopecky, K.E., 1970. Mycobacterium paratuberculosis in reproductive organs and semen of bulls. American journal of veterinary research 31, 255-258.

Larsen, A.B., Merkal, R.S., Vardaman, T.H., 1956. Survival time of Mycobacterium paratuberculosis. American journal of veterinary research 17, 549-551.

Larsen, A.B., Stalheim, O.H., Hughes, D.E., Appell, L.H., Richards, W.D., Himes, E.M., 1981. Mycobacterium paratuberculosis in the semen and genital organs of a semen-donor bull. Journal of the American Veterinary Medical Association 179, 169-171.

Laurin, E.L., Sanchez, J., Chaffer, M., McKenna, S.L., Keefe, G.P., 2017. Assessment of the relative sensitivity of milk ELISA for detection of Mycobacterium avium ssp. paratuberculosis infectious dairy cows. Journal of dairy science 100, 598607.

Lawrence, W.E., 1956. Congenital infection with M. paratuberculosis in cattle. Veterinary Record 68, 312-314.

Lensing, J.E., 1976. Intensity distribution patterns for five species of problem animals in south west africa. MADOQUA 10, 131-141.

Li, J., Macdonald, J., 2015. Advances in isothermal amplification: novel strategies inspired by biological processes. Biosensors \& bioelectronics 64, 196-211.

Li, L., Bannantine, J.P., Zhang, Q., Amonsin, A., May, B.J., Alt, D., Banerji, N., Kanjilal, S., Kapur, V., 2005. The complete genome sequence of Mycobacterium avium subspecies paratuberculosis. Proceedings of the National Academy of Sciences of the United States of America 102, 12344-12349. 
Lombard, J.E., 2011. Epidemiology and economics of paratuberculosis. The Veterinary clinics of North America. Food animal practice 27, 525-535.

Lombard, J.E., Wagner, B.A., Smith, R.L., McCluskey, B.J., Harris, B.N., Payeur, J.B., Garry, F.B., Salman, M.D., 2006. Evaluation of environmental sampling and culture to determine Mycobacterium avium subspecies paratuberculosis distribution and herd infection status on US dairy operations. Journal of dairy science $89,4163-4171$.

Ludlage, E., Mansfield, K., 2003. Clinical care and diseases of the common marmoset (Callithrix jacchus). Comparative medicine 53, 369-382.

Manning, E.J., Collins, M.T., 2001. Mycobacterium avium subsp. paratuberculosis: pathogen, pathogenesis and diagnosis. Revue scientifique et technique 20, 133150.

Manning, E.J., Kucera, T.E., Gates, N.B., Woods, L.M., Fallon-McKnight, M., 2003. Testing for Mycobacterium avium subsp. paratuberculosis infection in asymptomatic free-ranging tule elk from an infected herd. Journal of wildlife diseases 39, 323-328.

McClure, H.M., Chiodini, R.J., Anderson, D.C., Swenson, R.B., Thayer, W.R., Coutu, J.A., 1987. Mycobacterium paratuberculosis infection in a colony of stumptail macaques (Macaca arctoides). The Journal of infectious diseases 155, 10111019.

McDonald, W.L., Ridge, S.E., Hope, A.F., Condron, R.J., 1999. Evaluation of diagnostic tests for Johne's disease in young cattle. Australian veterinary journal 77, 113-119.

McFadden, J.J., Butcher, P.D., Chiodini, R., Hermon-Taylor, J., 1987. Crohn's diseaseisolated mycobacteria are identical to Mycobacterium paratuberculosis, as determined by DNA probes that distinguish between mycobacterial species. Journal of clinical microbiology 25, 796-801.

Merkal, R.S., 1973. Laboratory diagnosis of bovine paratuberculosis. Journal of the American Veterinary Medical Association 163, 1100-1102.

Merkal, R.S., 1984. Paratuberculosis: advances in cultural, serologic, and vaccination methods. Journal of the American Veterinary Medical Association 184, 939943. 
Merkal, R.S., Miller, J.M., Hintz, A.M., Bryner, J.H., 1982. Intrauterine inoculation of Mycobacterium paratuberculosis into guinea pigs and cattle. American journal of veterinary research $43,676-678$.

Michel, A.L., Bastianello, S.S., 2000. Paratuberculosis in sheep: an emerging disease in South Africa. Veterinary microbiology 77, 299-307.

Mijs, W., de Haas, P., Rossau, R., Van der Laan, T., Rigouts, L., Portaels, F., van Soolingen, D., 2002. Molecular evidence to support a proposal to reserve the designation Mycobacterium avium subsp. avium for bird-type isolates and ' $M$. avium subsp. hominissuis' for the human/porcine type of M. avium. International journal of systematic and evolutionary microbiology 52, 1505-1518.

Mitchell, R.M., Schukken, Y., Koets, A., Weber, M., Bakker, D., Stabel, J., Whitlock, R.H., Louzoun, Y., 2015. Differences in intermittent and continuous fecal shedding patterns between natural and experimental Mycobacterium avium subspecies paratuberculosis infections in cattle. Veterinary research 46, 66.

Momotani, E., Whipple, D.L., Thiermann, A.B., Cheville, N.F., 1988. Role of M cells and macrophages in the entrance of Mycobacterium paratuberculosis into domes of ileal Peyer's patches in calves. Veterinary pathology 25, 131-137.

Morris, U., Khamis, M., Aydin-Schmidt, B., Abass, A.K., Msellem, M.I., Nassor, M.H., Gonzalez, I.J., Martensson, A., Ali, A.S., Bjorkman, A., Cook, J., 2015. Field deployment of loop-mediated isothermal amplification for centralized massscreening of asymptomatic malaria in Zanzibar: a pre-elimination setting. Malaria Journal 14, 205.

Mortensen, H., Nielsen, S.S., Berg, P., 2004. Genetic variation and heritability of the antibody response to Mycobacterium avium subspecies paratuberculosis in Danish Holstein cows. Journal of dairy science 87, 2108-2113.

Mortier, R.A., Barkema, H.W., Bystrom, J.M., Illanes, O., Orsel, K., Wolf, R., Atkins, G., De Buck, J., 2013. Evaluation of age-dependent susceptibility in calves infected with two doses of Mycobacterium avium subspecies paratuberculosis using pathology and tissue culture. Veterinary research 44, 94.

Mortier, R.A., Barkema, H.W., Orsel, K., Wolf, R., De Buck, J., 2014. Shedding patterns of dairy calves experimentally infected with Mycobacterium avium subspecies paratuberculosis. Veterinary research 45, 71.

Motiwala, A.S., Amonsin, A., Strother, M., Manning, E.J., Kapur, V., Sreevatsan, S., 2004. Molecular epidemiology of Mycobacterium avium subsp. 
paratuberculosis isolates recovered from wild animal species. Journal of clinical microbiology 42, 1703-1712.

Mukamolova, G.V., Kaprelyants, A.S., Kell, D.B., Young, M., 2003. Adoption of the transiently non-culturable state -a bacterial survival strategy? Advances in microbial physiology 47, 65-129.

Münster, P., Fechner, K., Völkel, I., von Buchholz, A., Czerny, C.P., 2013a. Distribution of Mycobacterium avium ssp. paratuberculosis in a German zoological garden determined by IS900 semi-nested and quantitative real-time PCR. Veterinary microbiology 163, 116-123.

Münster, P., Völkel, I., Wemheuer, W., Schwarz, D., Döring, S., Czerny, C.P., 2013b. A longitudinal study to characterize the distribution patterns of Mycobacterium avium ssp. paratuberculosis in semen, blood and faeces of a naturally infected bull by IS 900 semi-nested and quantitative real-time PCR. Transboundary and emerging diseases 60, 175-187.

Naser, S.A., Ghobrial, G., Romero, C., Valentine, J.F., 2004. Culture of Mycobacterium avium subspecies paratuberculosis from the blood of patients with Crohn's disease. Lancet 364, 1039-1044.

Nebbia, P., Robino, P., Ferroglio, E., Rossi, L., Meneguz, G., Rosati, S., 2000. Paratuberculosis in red deer (Cervus elaphus hippelaphus) in the western Alps. Veterinary research communications 24, 435-443.

Nielsen, S.S., 2007. Danish control programme for bovine paratuberculosis. Cattle Practice 15, 161-168.

Nielsen, S.S., Toft, N., 2008. Ante mortem diagnosis of paratuberculosis: a review of accuracies of ELISA, interferon-gamma assay and faecal culture techniques. Veterinary microbiology 129, 217-235.

Nielsen, S.S., Toft, N., 2009. A review of prevalences of paratuberculosis in farmed animals in Europe. Preventive veterinary medicine 88, 1-14.

Norby, B., Fosgate, G.T., Manning, E.J., Collins, M.T., Roussel, A.J., 2007. Environmental mycobacteria in soil and water on beef ranches: association between presence of cultivable mycobacteria and soil and water physicochemical characteristics. Veterinary microbiology 124, 153-159.

Notomi, T., Okayama, H., Masubuchi, H., Yonekawa, T., Watanabe, K., Amino, N., Hase, T., 2000. Loop-mediated isothermal amplification of DNA. Nucleic acids research 28, E63. 
Oliver, J.D., 2010. Recent findings on the viable but nonculturable state in pathogenic bacteria. FEMS microbiology reviews 34, 415-425.

Olsen, I., Johansen, T.B., Billman-Jacobe, H., Nilsen, S.F., Djønne, B., 2004. A novel IS element, ISMpa1, in Mycobacterium avium subsp. paratuberculosis. Veterinary microbiology 98, 297-306.

Olsen, I., Sigurgardottir, G., Djonne, B., 2002. Paratuberculosis with special reference to cattle. A review. The Veterinary quarterly $24,12-28$.

Pavlik, I., Matlova, L., Bartl, J., Svastova, P., Dvorska, L., Whitlock, R., 2000. Parallel faecal and organ Mycobacterium avium subsp. paratuberculosis culture of different productivity types of cattle. Veterinary microbiology 77, 309-324.

Pearson, J.K., McClelland, T.G., 1955. Uterine infection and congenital Johne's disease in cattle. Veterinary Record 149, 339-369.

Pfyffer, G.E., Brown-Elliott, B.A., Wallace, R.J. 2015. Mycobacterium: general characteristics, laboratory detection, and staining procedures., In: Manual of Clinical Microbiology. American Society for Microbiology, 536-569.

Philpott, M., 1993. The dangers of disease transmission by artificial insemination and embryo transfer. The British veterinary journal 149, 339-369.

Piepenburg, O., Williams, C.H., Stemple, D.L., Armes, N.A., 2006. DNA detection using recombination proteins. PLoS biology 4, e204.

Polley, S.D., Gonzalez, I.J., Mohamed, D., Daly, R., Bowers, K., Watson, J., Mewse, E., Armstrong, M., Gray, C., Perkins, M.D., Bell, D., Kanda, H., Tomita, N., Kubota, Y., Mori, Y., Chiodini, P.L., Sutherland, C.J., 2013. Clinical evaluation of a loop-mediated amplification kit for diagnosis of imported malaria. The Journal of infectious diseases 208, 637-644.

Pribylova, R., Slana, I., Cech, S., Kralova, A., Pavlik, I., 2013. Mycobacterium avium subsp. paratuberculosis detected in the reproductive tract of cows from an infected herd. Reproduction in Domestic Animals 48, 790-794.

Raghuvanshi, S., Sharma, P., Singh, S., Van Kaer, L., Das, G., 2010. Mycobacterium tuberculosis evades host immunity by recruiting mesenchymal stem cells. Proceedings of the National Academy of Sciences of the United States of America 107, 21653-21658.

Raizman, E.A., Fetrow, J., Wells, S.J., Godden, S.M., Oakes, M.J., Vazquez, G., 2007. The association between Mycobacterium avium subsp. paratuberculosis fecal 
shedding or clinical Johne's disease and lactation performance on two Minnesota, USA dairy farms. Preventive veterinary medicine 78, 179-195.

Raizman, E.A., Wells, S.J., Godden, S.M., Bey, R.F., Oakes, M.J., Bentley, D.C., Olsen, K.E., 2004. The distribution of Mycobacterium avium ssp. paratuberculosis in the environment surrounding Minnesota dairy farms. Journal of dairy science $87,2959-2966$.

Reichel, M.P., Kittelberger, R., Penrose, M.E., Meynell, R.M., Cousins, D., Ellis, T., Mutharia, L.M., Sugden, E.A., Johns, A.H., de Lisle, G.W., 1999. Comparison of serological tests and faecal culture for the detection of Mycobacterium avium subsp. paratuberculosis infection in cattle and analysis of the antigens involved. Veterinary microbiology 66, 135-150.

Ren, H., Yang, M., Zhang, G., Liu, S., Wang, X., Ke, Y., Du, X., Wang, Z., Huang, L., Liu, C., Chen, Z., 2016. Development of a rapid recombinase polymerase amplification assay for detection of Brucella in blood samples. Molecular and cellular probes $30,122-124$.

Ridge, S.E., Harkin, J.T., Badman, R.T., Mellor, A.M., Larsen, J.W., 1995. Johne's disease in alpacas (Lama pacos) in Australia. Australian veterinary journal 72, 150-153.

Rindi, L., Garzelli, C., 2014. Genetic diversity and phylogeny of Mycobacterium avium. Infection, genetics and evolution 21, 375-383.

Robbe-Austerman, S., Krull, A., Stabel, J., 2006. Time delay, temperature effects and assessment of positive controls on whole blood for the gamma interferon ELISA to detect paratuberculosis. Journal of Veterinary Medicine, Series B 53, 213217.

Robino, P., Nebbia, P., Tramuta, C., Martinet, M., Ferroglio, E., De Meneghi, D., 2008. Identification of Mycobacterium avium subsp. paratuberculosis in wild cervids (Cervus elaphus hippelaphus and Capreolus capreolus) from Northwestern Italy. European Journal of Wildlife Research 54, 357-360.

Rohde, R.F., Shulaw, W.P., 1990. Isolation of Mycobacterium paratuberculosis from the uterine flush fluids of cows with clinical paratuberculosis. Journal of the American Veterinary Medical Association 197, 1482-1483.

Rolle, M., Mayr, A., 2002. Medical microbiology, infection and epidemics [Medizinische Mikrobiologie, Infektions- und Seuchenlehre]. Enke Verlag. 
REFERENCES

Rosenberger, G., 1978. Infectious diseases of the digestive system [Infektionskrankheiten des Verdauungsapparates]. In: Krankheiten des Rindes 2, Rosenberg, G. (Eds.), Verlag Parey, 756-760.

Rowe, M.T., Grant, I.R., 2006. Mycobacterium avium ssp. paratuberculosis and its potential survival tactics. Letters in applied microbiology 42, 305-311.

Rudoler, N., 2004. Is Mycobacterium avium subsp. paratuberculosis an etiological factor in Crohn's disease. Israel Journal of Veterinary Medicine 59, 60-67.

Sasikala, M., Reddy, D.N., Pratap, N., Sharma, S.K., Balkumar, P.R., Sekaran, A., Banerjee, R., Reddy, D.B., 2009. Absence of Mycobacterium avium ss paratuberculosis-specific IS900 sequence in intestinal biopsy tissues of Indian patients with Crohn's disease. Indian journal of gastroenterology 28, 169-174.

Schwartz, D., Shafran, I., Romero, C., Piromalli, C., Biggerstaff, J., Naser, N., Chamberlin, W., Naser, S., 2000. Use of short-term culture for identification of Mycobacterium avium subsp. paratuberculosis in tissue from Crohn's disease patients. Clinical Microbiology and Infection 6, 303-307.

Sechi, L.A., Scanu, A.M., Molicotti, P., Cannas, S., Mura, M., Dettori, G., Fadda, G., Zanetti, S., 2005. Detection and isolation of Mycobacterium avium subspecies paratuberculosis from intestinal mucosal biopsies of patients with and without Crohn's disease in Sardinia. The American journal of gastroenterology 100, 1529-1536.

Seitz, S.E., Heider, L.E., Heuston, W.D., Bech-Nielsen, S., Rings, D.M., Spangler, L., 1989. Bovine fetal infection with Mycobacterium paratuberculosis. Journal of the American Veterinary Medical Association 194, 1423-1426.

Sharifzadeh, A., Doosti, A., Fazeli, M.H., Adavoudi, I., 2010. Nested PCR on semen samples for the detection of Mycobacterium avium subsp paratuberculosis. African Journal of Microbiology Research 4, 2787-2789.

Shaughnessy, L.J., Smith, L.A., Evans, J., Anderson, D., Caldow, G., Marion, G., Low, J.C., Hutchings, M.R., 2013. High prevalence of paratuberculosis in rabbits is associated with difficulties in controlling the disease in cattle. Veterinary journal 198, 267-270.

Sherman, D., Gay, J., Bouley, D., Nelson, G., 1990. Comparison of the complementfixation and agar gel immunodiffusion tests for diagnosis of subclinical bovine paratuberculosis. American journal of veterinary research 51, 461-465. 
Sherman, D., Markham, R., Bates, F., 1984. Agar gel immunodiffusion test for diagnosis of clinical paratuberculosis in cattle. Journal of the American Veterinary Medical Association 185, 179-182.

Sigurdardottir, O.G., Bakke-McKellep, A.M., Djonne, B., Evensen, O., 2005. Mycobacterium avium subsp. paratuberculosis enters the small intestinal mucosa of goat kids in areas with and without Peyer's patches as demonstrated with the everted sleeve method. Comparative immunology, microbiology and infectious diseases 28, 223-230.

Singh, S.V., Singh, A.V., Singh, P.K., Kumar, A., Singh, B., 2011. Molecular identification and characterization of Mycobacterium avium subspecies paratuberculosis in free living non-human primate (Rhesus macaques) from North India. Comparative immunology, microbiology and infectious diseases 34, 267-271.

Sivakumar, P., Tripathi, B.N., Singh, N., 2005. Detection of Mycobacterium avium subsp. paratuberculosis in intestinal and lymph node tissues of water buffaloes (Bubalus bubalis) by PCR and bacterial culture. Veterinary microbiology 108, 263-270.

Smith, H.W., 1960. The examination of milk for the presence of Mycobacterium johnei. The Journal of Pathology and Bacteriology 80, 440.

Sockett, D., Carr, D., Collins, M., 1992. Evaluation of conventional and radiometric fecal culture and a commercial DNA probe for diagnosis of Mycobacterium paratuberculosis infections in cattle. Canadian Journal of Veterinary Research $56,148$.

Sohal, J.S., Singh, S.V., Singh, P.K., Singh, A.V., Kumar, N., 2013. A new marker IS1311 L2 PCR-REA for identification of 'Indian Bison' type Mycobacterium avium subspecies paratuberculosis. Indian Journal of Biotechnology 12, 204207.

Spahr, U., Schafroth, K., 2001. Fate of Mycobacterium avium subsp. paratuberculosis in Swiss hard and semihard cheese manufactured from raw milk. Applied and environmental microbiology 67, 4199-4205.

Stabel, J.R., 1996. Production of gamma-interferon by peripheral blood mononuclear cells: an important diagnostic tool for detection of subclinical paratuberculosis. Journal of veterinary diagnostic investigation 8, 345-350.

Stabel, J.R., 1998. Johne's disease: a hidden threat. Journal of dairy science 81, 283-288. 
Stabel, J.R., Bannantine, J.P., 2005. Development of a nested PCR method targeting a unique multicopy element, ISMap02, for detection of Mycobacterium avium subsp. paratuberculosis in fecal samples. Journal of clinical microbiology 43, 4744-4750.

Stabel, J.R., Kimura, K., Robbe-Austerman, S., 2007. Augmentation of secreted and intracellular gamma interferon following johnin purified protein derivative sensitization of cows naturally infected with Mycobacterium avium subsp. paratuberculosis. Journal of Veterinary Diagnostic Investigation 19, 43-51.

Stanley, E.C., Mole, R.J., Smith, R.J., Glenn, S.M., Barer, M.R., McGowan, M., Rees, C.E., 2007. Development of a new, combined rapid method using phage and PCR for detection and identification of viable Mycobacterium paratuberculosis bacteria within 48 hours. Applied and environmental microbiology 73, 18511857.

Stevenson, K., 2015. Genetic diversity of Mycobacterium avium subspecies paratuberculosis and the influence of strain type on infection and pathogenesis: a review. Veterinary research 46, 64.

Stief, B., Möbius, P., Turk, H., Horugel, U., Arnold, C., Pohle, D., 2012. Paratuberculosis in a miniature donkey (Equus asinus f. asinus) [Paratuberkulose in einem Miniatur-Esel (Equus asinus f. asinus)]. Berliner und Münchener tierärztliche Wochenschrift 125, 38-44.

Streeter, R.N., Hoffsis, G.F., Bech-Nielsen, S., Shulaw, W.P., Rings, D.M., 1995. Isolation of Mycobacterium paratuberculosis from colostrum and milk of subclinically infected cows. American journal of veterinary research 56, 13221324.

Strommenger, B., Stevenson, K., Gerlach, G.-F., 2001. Isolation and diagnostic potential of ISMav2, a novel insertion sequence-like element from Mycobacterium avium subspecies paratuberculosis. FEMS Microbiology Letters 196, 31-37.

Sweeney, R.W., 1996. Transmission of paratuberculosis. The Veterinary clinics of North America. Food animal practice 12, 305-312.

Sweeney, R.W., Whitlock, R.H., McAdams, S., Fyock, T., 2006. Longitudinal study of ELISA seroreactivity to Mycobacterium avium subsp. paratuberculosis in infected cattle and culture-negative herd mates. Journal of veterinary diagnostic investigation 18, 2-6. 
Sweeney, R.W., Whitlock, R.H., Rosenberger, A.E., 1992a. Mycobacterium paratuberculosis Cultured from Milk and Supramammary Lymph-Nodes of Infected Asymptomatic Cows. Journal of clinical microbiology 30, 166-171.

Sweeney, R.W., Whitlock, R.H., Rosenberger, A.E., 1992b. Mycobacterium paratuberculosis isolated from fetuses of infected cows not manifesting signs of the disease. American journal of veterinary research 53, 477-480.

Swift, B.M., Denton, E.J., Mahendran, S.A., Huxley, J.N., Rees, C.E., 2013. Development of a rapid phage-based method for the detection of viable Mycobacterium avium subsp. paratuberculosis in blood within 48 h. Journal of microbiological methods 94, 175-179.

Taddei, S., Robbi, C., Cesena, C., Rossi, I., Schiano, E., Arrigoni, N., Vicenzoni, G., Cavirani, S., 2004. Detection of Mycobacterium Avium Subsp. Paratuberculosis in Bovine Fecal Samples: Comparison of Three Polymerase Chain ReactionBased Diagnostic Tests with a Conventional Culture Method. Journal of veterinary diagnostic investigation $16,503-508$.

Taylor, T.K., Wilks, C.R., McQueen, D.S., 1981. Isolation of Mycobacterium paratuberculosis from the milk of a cow with Johne's disease. The Veterinary record $109,532-533$.

Telugu, B.P., Green, J.A., 2008. Comparative Placentation. In: Comparative reproductive biology. Schatten, H., Constantinescu, G. M. (Eds.), Blackwell Publishing, 271-320.

Thibault, V.C., Bryant , J., Harris, S., Bentley, S., Smith, D.G.E., McLuckie, J., Parkhill, J., Stevenson, K. 2012. Genome sequencing to identify phylogenetic diversity and single nucleotide polymorphisms in Mycobacterium avium subspecies paratuberculosis. In: Proceedings of the 11th International Colloquium on Paratuberculosis, Sydney (Australia), 267.

Thorel, M.F., Krichevsky, M., Levy-Frebault, V.V., 1990. Numerical taxonomy of mycobactin-dependent mycobacteria, emended description of Mycobacterium avium, and description of Mycobacterium avium subsp. avium subsp. nov., Mycobacterium avium subsp. paratuberculosis subsp. nov., and Mycobacterium avium subsp. silvaticum subsp. nov. International journal of systematic bacteriology 40, 254-260.

Tortoli, E., 2014. Microbiological features and clinical relevance of new species of the genus Mycobacterium. Clinical microbiology reviews 27, 727-752. 
Tortoli, E., Rindi, L., Garcia, M.J., Chiaradonna, P., Dei, R., Garzelli, C., Kroppenstedt, R.M., Lari, N., Mattei, R., Mariottini, A., Mazzarelli, G., Murcia, M.I., Nanetti, A., Piccoli, P., Scarparo, C., 2004. Proposal to elevate the genetic variant MACA, included in the Mycobacterium avium complex, to species rank as Mycobacterium chimaera sp. nov. International journal of systematic and evolutionary microbiology 54, 1277-1285.

Tunkle, B., Aleraj, Z., 1965. The recovering of M. johnei in the semen of bull from one station for artificial insemination. Veterinarski glasnik 19, 845-849.

Turenne, C.Y., Alexander, D.C., 2010. Mycobacterium avium complex. In: Paratuberculosis: Organism, Disease, Control. Behr, M. A., \& Collins, D. M. (Eds.), CABI., 60-72.

Twort, F.W., Ingram, G.L.Y., 1912. A method for isolating and cultivating the Mycobacterium enteritidis chronicae pseudotuberculosae bovis johne and some experiments on the preparation of a diagnostic vaccine for pseudotuberculous enteritis of bovines. Proceedings of the Royal Society 84, 517-543.

Valentin-Weigand, P., Goethe, R., 1999. Pathogenesis of Mycobacterium avium subspecies paratuberculosis infections in ruminants: still more questions than answers. Microbes and infection 1, 1121-1127.

van Ingen, J., Boeree, M.J., Kosters, K., Wieland, A., Tortoli, E., Dekhuijzen, P.N., van Soolingen, D., 2009. Proposal to elevate Mycobacterium avium complex ITS sequevar MAC-Q to Mycobacterium vulneris sp. nov. International journal of systematic and evolutionary microbiology 59, 2277-2282.

Van Kruiningen, H.J., 1999. Lack of support for a common etiology in Johne's disease of animals and Crohn's disease in humans. Inflammatory bowel diseases 5, 183191.

Van Ness, J., Van Ness, L.K., Galas, D.J., 2003. Isothermal reactions for the amplification of oligonucleotides. Proceedings of the National Academy of Sciences of the United States of America 100, 4504-4509.

van Roermund, H.J., Bakker, D., Willemsen, P.T., de Jong, M.C., 2007. Horizontal transmission of Mycobacterium avium subsp. paratuberculosis in cattle in an experimental setting: calves can transmit the infection to other calves. Veterinary microbiology 122, 270-279. 
Vansnick, E., de Rijk, P., Vercammen, F., Geysen, D., Rigouts, L., Portaels, F., 2004. Newly developed primers for the detection of Mycobacterium avium subspecies paratuberculosis. Veterinary microbiology 100, 197-204.

Vincent, M., Xu, Y., Kong, H., 2004. Helicase-dependent isothermal DNA amplification. EMBO reports 5, 795-800.

Ward, M.P., Perez, A.M., 2004. Association between soil type and paratuberculosis in cattle herds. American journal of veterinary research 65, 10-14.

Weber, M., Schaik, G.v. 2008. Results of the Dutch bulk milk quality assurance programme for paratuberculosis. In: Proceedings of the 9th International Colloquium on Paratuberculosis, Tsukuba (Japan), 29 October-2 November 2007, 324-327.

Whipple, D.L., Callihan, D.R., Jarnagin, J.L., 1991. Cultivation of Mycobacterium paratuberculosis from bovine fecal specimens and a suggested standardized procedure. Journal of Veterinary Diagnostic Investigation 3, 368-373.

Whipple, D.L., Kapke, P.A., Andersen, P.R., 1992. Comparison of a commercial DNA probe test and three cultivation procedures for detection of Mycobacterium paratuberculosis in bovine feces. Journal of Veterinary Diagnostic Investigation 4, 23-27.

Whitlock, R., Wells, S., Sweeney, R.W., Van Tiem, J., 2000. ELISA and fecal culture for paratuberculosis (Johne's disease): sensitivity and specificity of each method. Veterinary microbiology 77, 387-398.

Whitlock, R.H., Buergelt, C., 1996. Preclinical and clinical manifestations of paratuberculosis (including pathology). The Veterinary clinics of North America. Food animal practice 12, 345-356.

Whittington, R.J., Marsh, I.B., Reddacliff, L.A., 2005. Survival of Mycobacterium avium subsp. paratuberculosis in dam water and sediment. Applied and environmental microbiology 71, 5304-5308.

Whittington, R.J., Marshall, D.J., Nicholls, P.J., Marsh, I.B., Reddacliff, L.A., 2004. Survival and dormancy of Mycobacterium avium subsp. paratuberculosis in the environment. Applied and environmental microbiology 70, 2989-3004.

Whittington, R.J., Windsor, P.A., 2009. In utero infection of cattle with Mycobacterium avium subsp. paratuberculosis: a critical review and meta-analysis. Veterinary journal 179, 60-69. 
Wilks, C.R., Taylor, T.K., Russell, E.G., Thomas, T.R., 1981. Isolation of mycobacteria inducing cross-reactions in the complement fixation test for Johne's disease. Research in veterinary science $30,323-327$.

Zavgorodniy, A., Pozmogova, S., Girka, M., Goncharova, N., 2015. Isolation of Mycobacterium avium subspecies paratuberculosis from zoo animals. Journal for veterinary medicine, biotechnology and biosafety 1, 17-19.

Zwick, L.S., Walsh, T.F., Barbiers, R., Collins, M.T., Kinsel, M.J., Murnane, R.D., 2002. Paratuberculosis in a mandrill (Papio sphinx). Journal of veterinary diagnostic investigation 14, 326-328. 
APPENDIX

5 APPENDIX 


\section{LIST OF PUBLICATIONS}

This thesis is based on the work contained in the following papers:

I FECHNER K., SCHÄFER, J., WIEGEL, C., LUDWIG, J., MÜNSTER, P., SHARIFI, A. R., WEMHEUER, W., CZERNY, C.-P. (2015): Distribution of Mycobacterium avium subsp. paratuberculosis in a Subclinical Naturally Infected German Fleckvieh Bull. Transboundary and emerging diseases, 64(3), 916-928.

II FECHNER K., MÄTZ-RENSING, K., LAMPE, K., KAUP, F.-J., CZERNY, C.P., SCHÄFER, J. (2017): Detection of Mycobacterium avium subsp. paratuberculosis by IS900 based PCR assays in non-human primates kept in Germany. Journal of medical primatology, DOI 10.1111/jmp.12270.

III FECHNER K., SCHÄFER, J., MÜNSTER, P., TERNES, K., DÖRING, S., VÖLKEL, I., KAUP, F.-J., CZERNY, C.-P. (2017): Detection of Mycobacterium avium subsp. paratuberculosis in rock hyraxes (Procavia capensis) imported from South Africa. Journal of Zoo and Wildlife Medicine, under review.

IV HANSEN, S., SCHÄFER, J., FECHNER, K., CZERNY, C.-P., ABD EL WAHED, A. (2016): Development of a recombinase polymerase amplification assay for rapid detection of the Mycobacterium avium subsp. paratuberculosis. Plos one, 11(12), e0168733. 


\section{LIST OF PRESENTATIONS}

I 5. Arbeitstagung des nationalen Referenzlabors für Tuberkulose und des nationalen Referenzlabors für Paratuberkulose (29. - 30.01.2013 in Jena):

FECHNER, K., VÖLKEL, I., MÜNSTER, P., V. BUCHHOLZ, A., WEMHEUER, W., CZERNY, C.-P.: "Vergleichende molekularbiologische Analysen von Mycobacterium avium ssp. paratuberulosis-Isolaten aus Hauswiederkäuern und Zootieren."

II 5. Arbeitstagung des nationalen Referenzlabors für Tuberkulose und des nationalen Referenzlabors für Paratuberkulose (29. - 30.01.2013 in Jena):

VÖLKEL, I., SCHWARZ, D., SCHÜTTLER, J., WESTERNHORST, U., MÜNSTER, P., FECHNER, K., WEMHEUER, W., CZERNY, C.-P.: "Zytologische und hämatologische Parameter im Verlauf natürlicher Infektionen mit Mycobacterium avium ssp. paratuberculosis bei Besamungsbullen.”

III DVG VET-Congress "Tagung der DVG-Fachgruppe Umwelt- und Tierhygiene" (06. - 10.09.2013 in Berlin):

FECHNER, K., WEMHEUER, W., CZERNY, C.-P.: "Epidemiologische Untersuchungen zum Vorkommen von Mycobacterium avium ssp. paratuberculosis bei Hauswiederkäuern und Zootieren mittels IS900 basierten PCR-Verfahren.”

IV 34. Tagung der DVG-Fachgruppe AVID "Veterinärmedizinische Infektionsdiagnostik; Schwerpunkt Bakteriologie"(09. - 11.09.2015 in Banz):

FECHNER, K., SCHÄFER, J., BURCHARDT, S., SCHÖTTKER-WEGNER, H.H., WERCKENTHIN, C., NORDHOFF, M., KLARMANN, D., WEMHEUER W., CZERNY, C.-P.: "Nachweis von Mycobacterium avium ssp. paratuberculosis in subklinisch infizierten Bullen" 
V National Symposium on Zoonose Research 2016 (13. - 14.10.2016 in Berlin):

FECHNER, K., MÄTZ-RENSING, K., LAMPE, K., KAUP, F.-J., CZERNY, C.P., SCHÄFER, J.: “Mycobacterium avium ssp. paratuberculosis in non-human primates" 


\section{LIST OF POSTERS}

I 12 ${ }^{\text {th }}$ International Colloquium on Paratuberculosis (22. - 26.06.2014, Parma, Italy): FECHNER, K., SCHÄFER, J., WEMHEUER, W., CZERNY, C. P.: "Detectability of Mycobacterium avium ssp. paratuberculosis in subclinically infected bulls."

II 34. Tagung der DVG-Fachgruppe AVID "Veterinärmedizinische Infektionsdiagnostik; Schwerpunkt Bakteriologie " (09. - 11.09.2015 in Banz): FECHNER, K., MÄTZ-RENSING, K., LAMPE, K., KAUP, F.-J., CZERNY, C.P., SCHÄFER, J.: "Nachweis von Mycobacterium avium ssp. paratuberculosis in Gewebeproben von nicht humanen Primaten." 


\section{CURRICULUM VITAE}

\section{Kim Fechner}

01 July 1987 in Celle

\section{Work experience}

Since 09/2012 Division of Microbiology and Animal Hygiene, Institute of

Veterinary Medicine, Department of Animal Sciences, Faculty of

Agricultural Sciences, Georg-August-University Göttingen,

Germany

Position: Research assistant

05/2012 - 09/2012 Gesellschaft für Innovative Veterinärdiagnostik (IVD GmbH), Hannover

Position: Research assistant

\section{$\underline{\text { University }}$}

Since 09/2012 Georg-August-University Göttingen, Germany

Ph.D. program: Agricultural sciences

Major: Animal sciences

04/2010 - 06/2012 Georg-August-University Göttingen, Germany

Master's program: Agricultural sciences

Major: Animal sciences

Graduation: Master of Science

10/2006 - 03/2010 Georg-August-University Göttingen, Germany

Master's program: Agricultural sciences

Major: Animal sciences

Graduation: Bachelor of Science 


\section{ACKNOWLEDEMENTS}

The studies forming the basis of this thesis were undertaken at the German GeorgAugust-University Göttingen, Department of Animal Sciences, Division of Microbiology and Animal Hygiene.

First of all, I would like to thank my academic supervisor, Prof. Dr. Dr. Claus-Peter Czerny, for this research and work opportunity and for his continuous support. I am also thankful to Prof. Jürgen Hummel and Prof. Engel Hessel, for accepting and assuming the co- supervisors of my thesis.

I am particularly grateful to Henrike Ahsendorf, Stephani Fischer, Franziska Pilger and Christoph Braun for their help and friendship, especially during difficult times. Furthermore, I thank all my former and current colleagues and employees at the Division of Microbiology and Animal Hygiene. My special thanks go to Julia Christine Steinfeldt, Caroline Bierschenk, Nicole Graefe, Martin Seeländer, Tina Althaus, Thomas Kinder and Wilhelm Wemheuer for working with me and assisting me with these investigations.

I am very grateful to Ahmed Abd El Wahed and Inger Völkel for their helpful scientific discussions and constant encouragement.

Many thanks also go to Karen Lampe, Kerstin Mätz-Rensing and Prof. Dr. FranzJosef Kaup from the Pathology Unit, German Primate Centre (DPZ), Göttingen, for supplying the non-human primate samples, scientific discussion and assistance in completing the publication manuscripts. I am grateful in every possible way and hope to maintain our collaboration in the future.

I am also very thankful to Jenny Schäfer, for her understanding, encouragement, valuable advice, useful discussions, motivation ("chakka du schaffst das") and for her critical review of my manuscripts.

I would like to thank my friends, Elisa Senger, Johanna Rabe and Regina Ryll, who have accompanied and helped me unwaveringly during the past years. 
Finally, I would like to thank my family, especially my parents, Thomas und Martina Fechner, and Philipp Gottschalk, for their constant support not only throughout writing this thesis but also throughout my whole life. 


\section{DECLARATION}

1. I, hereby, declare that this Ph.D. dissertation has not been presented to any other examination body either in its present or a similar form.

Furthermore, I also affirm that I have not applied for a Ph.D. at any other higher school of education.

Göttingen,

(Signature)

(Name in block capitals)

2. I, hereby, solemnly declare that this dissertation was undertaken independently and without any unauthorised aid.

Göttingen,

(Signature)

(Name in block capitals) 